\title{
A STUDY OF HOT TEARING DURING SOLIDIFICATION OF B206 ALUMINUM ALLOY
}

\author{
by \\ Francesco D'Elia \\ Bachelor of Engineering, Ryerson University, 2007 \\ Master of Applied Science, Ryerson University, 2009
}

\author{
A dissertation \\ presented to Ryerson University \\ in partial fulfillment of the \\ requirements for the degree of
}

Doctor of Philosophy

in the Program of

Mechanical and Industrial Engineering

Toronto, Ontario, Canada, 2015

(C) Francesco D’Elia 2015 


\section{AUTHOR'S DECLARATION FOR SUBMISSION OF ELECTRONIC DISSERTATION}

I hereby declare that I am the sole author of this dissertation. This is a true copy of the dissertation, including any required final revisions, as accepted by my examiners.

I authorize Ryerson University to lend this dissertation to other institutions or individuals for the purpose of scholarly research.

I further authorize Ryerson University to reproduce this dissertation by photocopying or by other means, in total or in part, at the request of other institutions or individuals for the purpose of scholarly research.

I understand that my dissertation may be made electronically available to the public. 


\title{
Abstract
}

\section{A STUDY OF HOT TEARING DURING SOLIDIFICATION OF B206 ALUMINUM ALLOY}

\author{
Doctor of Philosophy, 2015 \\ Francesco D'Elia \\ Mechanical and Industrial Engineering \\ Ryerson University
}

\begin{abstract}
Aluminum-copper $(\mathrm{Al}-\mathrm{Cu})$ alloy B206 is a high strength and ductile alloy showing promise for use in automotive suspension components. Incorporation of lightweight B206 alloy in automotive suspension components may significantly reduce overall vehicle weight and increase the vehicle's fuel efficiency. However, one of the major factors inhibiting the use of B206 is its high susceptibility to hot tearing during casting. Hot tearing is a complex phenomenon attributed to alloy solidification, microstructure and stress/strain development within a casting. Numerous methods (e.g. preheating of mold, grain refinement, elimination of sharp corners in a component) help to reduce the occurrence of hot tears in castings, but the underlying mechanisms responsible for hot tearing remain ambiguous. This research aims to advance the understanding of the mechanisms responsible for hot tearing in B206 Al alloy.
\end{abstract}

In this research, the conditions associated with the formation of hot tears in B206 were investigated via ex situ and in situ methods. Titanium was added in three levels (i.e. unrefined, 0.02 and $0.05 \mathrm{wt} \%$ ) to investigate the effect of grain refinement on hot tearing. Ex situ neutron diffraction strain mapping was carried out on the three B206 castings to determine casting strain and stress. Further, in situ techniques were used to establish the onset temperature and solid fraction of hot tearing in B206 and to improve the understanding of microstructure development in B206.

The results indicate that titanium additions had a significant impact on the hot tearing susceptibility of B206, by effectively reducing grain size and transforming grain morphology from coarse dendrites to fine globular grains. Further, thermal analysis suggested that grain 
refinement delayed the onset of dendrite coherency in B206 and therefore enhanced the duration of bulk liquid metal feeding for the refined casting conditions. As a result, the interactive effects of such factors resulted in a more uniform distribution of strain, and subsequent higher resistance to hot tearing for the grain refined castings. Finally, in situ analysis determined the onset solid fraction of hot tearing in B206 and provided an understanding of the role of microstructure on hot tearing in B206. 


\section{Acknowledgements}

I would like to express my deepest gratitude to my supervisor, mentor and "academic father", Prof. Ravindran, for his guidance, patience and continuous support during my graduate studies.

I am extremely thankful to Dr. Dimitry Sediako for his direction with the neutron diffraction experiments and analysis.

I am also grateful to Professors Alex McLean and David Naylor for their valuable input and suggestions.

I would like to thank Mr. Alan Machin for his advice and contribution of knowledge.

I am also thankful to Mr. Joseph Amankrah for technical assistance and stimulating discussions.

I am grateful to Dr. Sophie Lun Sin, Mr. Abdallah Elsayed, Mr. Anthony Lombardi and the members of the Centre for Near-net-shape Processing of Materials for their help with casting experiments.

I am also thankful to Dr. Norbert Hort, Mr. Günter Meister and Ms. Zhi Wang of the HelmholtzZentrum Geesthacht for help with in situ casting experiments.

I would also like to acknowledge Natural Science and Engineering Research Council of Canada (NSERC) for the Canada Graduate Doctoral Scholarship.

I am grateful to Mr. Tom Warren of Gamma Foundries for chemical analysis of samples.

Finally, I would like to thank my family and those who have been close to me during this academic journey. Without their support and patience, completion of this dissertation would not have been possible. 


\section{Table of Contents}

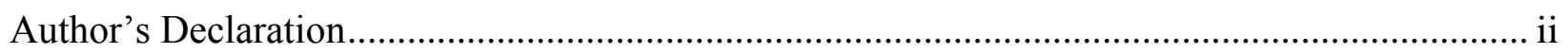

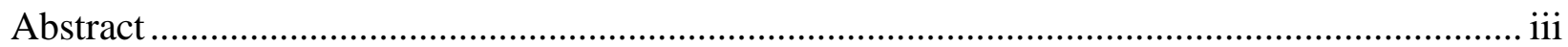

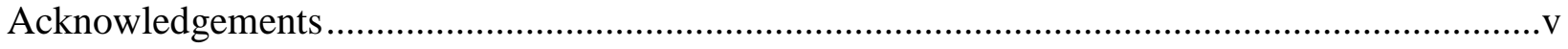

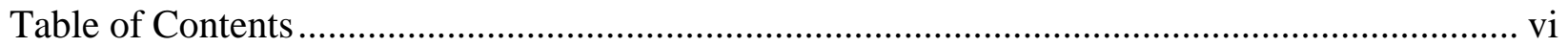

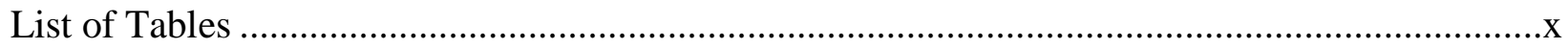

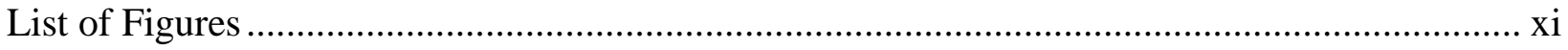

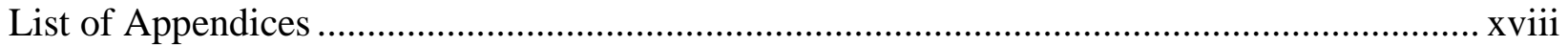

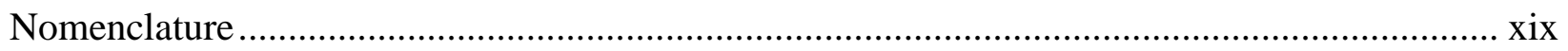

Chapter 1 - Introduction ........................................................................................................................1

Chapter 2 - Literature Review ............................................................................................................5

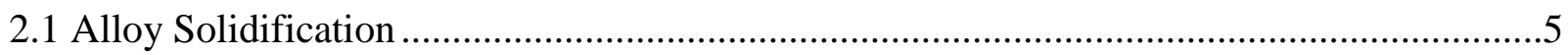

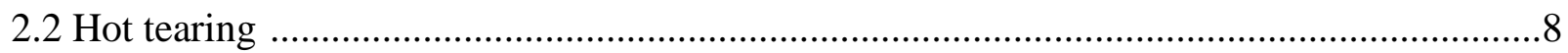

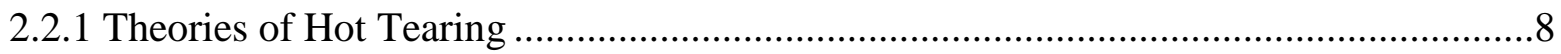

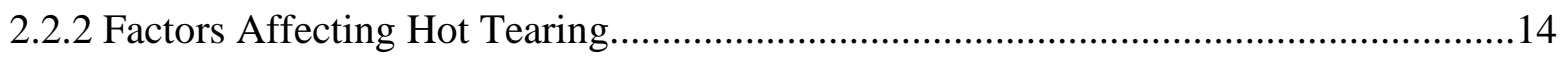

2.2.2.1 Effect of Alloy Composition .......................................................................14

2.2.2.2 Effect of Grain Size and Morphology ...............................................................19

2.2.2.3 Effect of Casting Process Parameters ..............................................................24

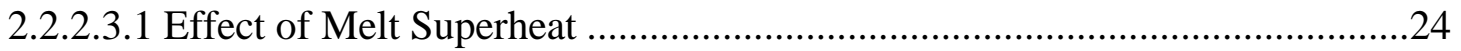

2.2.2.3.2 Effect of Mold Temperature .....................................................................25

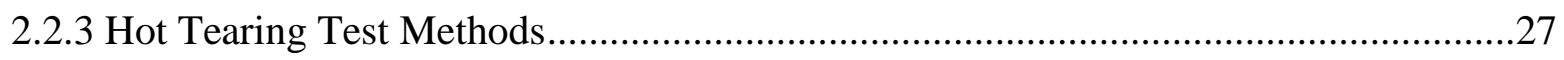

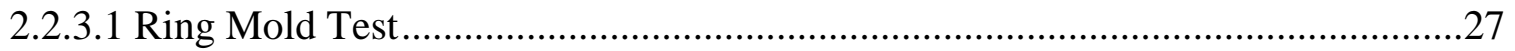

2.2.3.2 "Dog-Bone" Mold Test ..................................................................................28

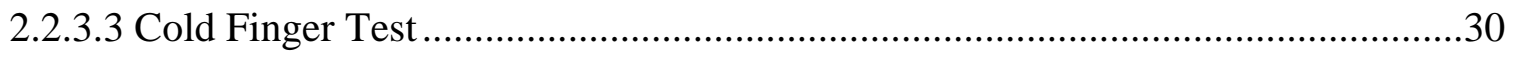

2.2.3.4 In Situ Methods.................................................................................................32

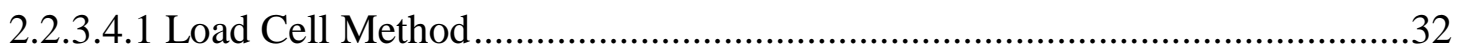

2.2.3.4.2 Linear Variable Differential Transformer (LVDT) Method ...........................36

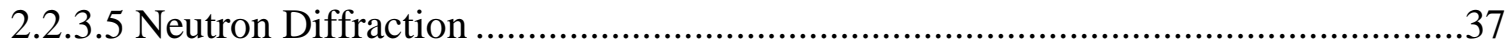

2.2.3.5.1 Measurement of Residual Strain and Stress by Neutron Diffraction .............39

2.2.3.5.2 Analysis of Hot Tearing by Neutron Diffraction ...........................................41 
Chapter 3 - Experimental Procedure .43

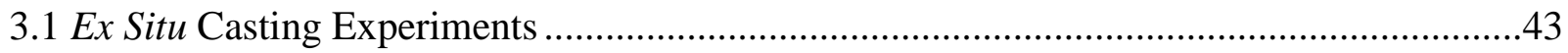

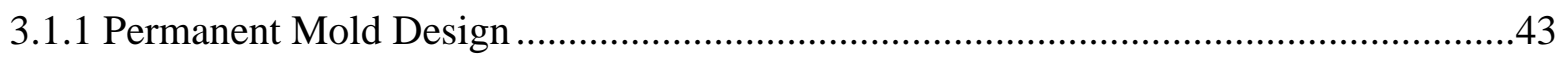

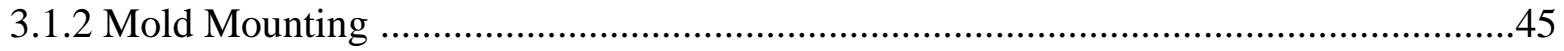

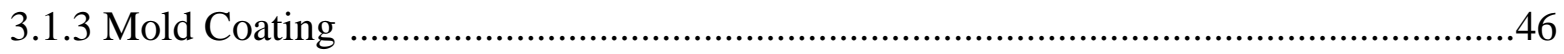

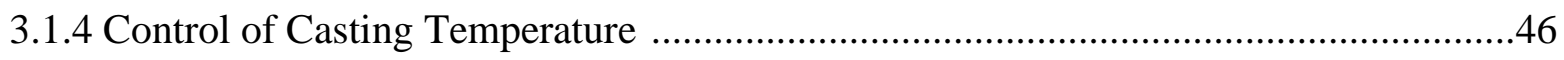

3.1.5 Alloy Melting and Casting .........................................................................................4

3.2 Neutron Diffraction Strain Mapping Experiments ............................................................49

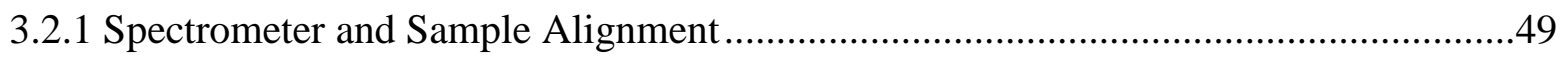

3.2.2 Neutron Beam Sampling Volume .......................................................................

3.2.3 Selection of Samples for Strain Analysis ………….................................................53

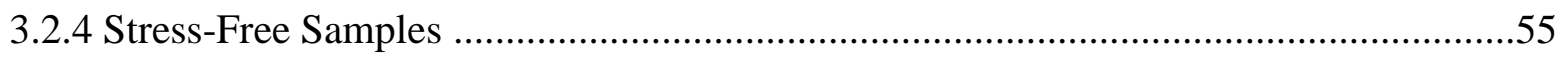

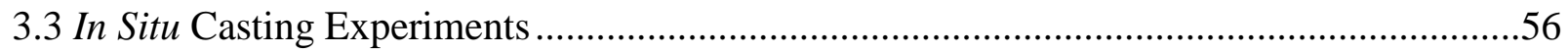

3.3.1 Hot Tearing Apparatus .......................................................................................56

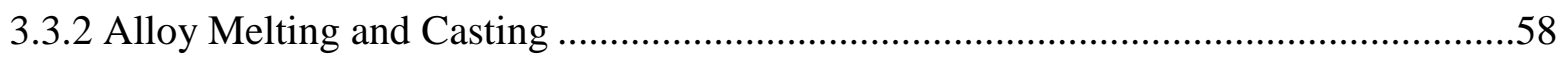

3.3.3 Determination of Onset Temperature of Hot Tearing ................................................59

3.4 In Situ Neutron Diffraction Solidification Analysis ............................................................60

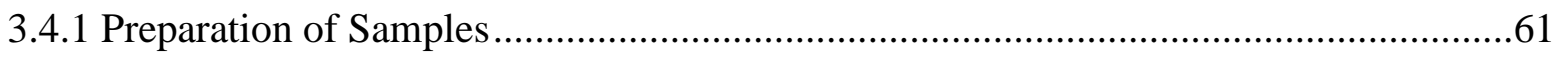

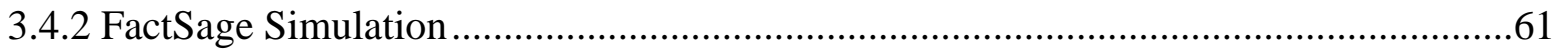

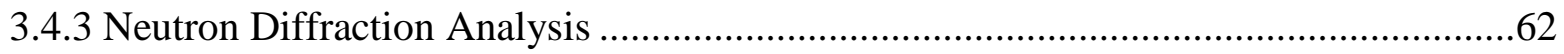

3.5 Casting Microstructure Analysis ................................................................................64

Chapter 4 - Ex Situ Analysis of Hot Tearing in B206 ....................................................................66

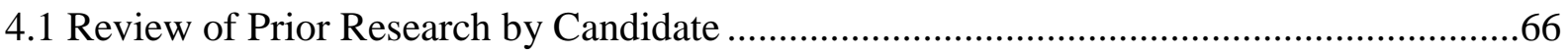

4.2 Observation of Hot Tears on Casting Surfaces............................................................67

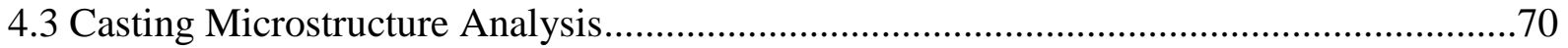

4.3.1 Al-Cu Phase Diagram ……………………………….....................................

4.3.2 Grain Size and Morphology .................................................................................

4.3.3 General Alloy Microstructure …………………...................................................72

4.3.4 Analysis of Hot Tear Regions .........................................................................78

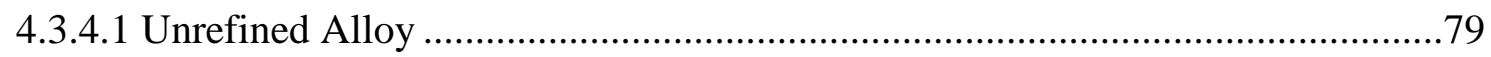

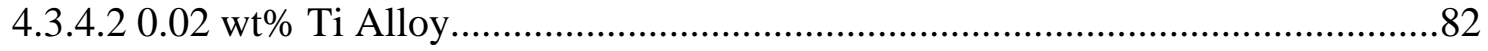




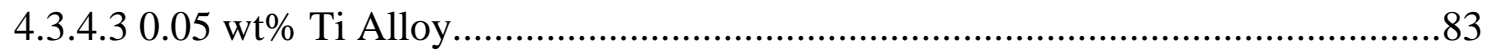

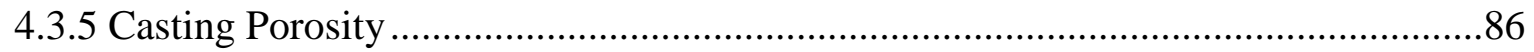

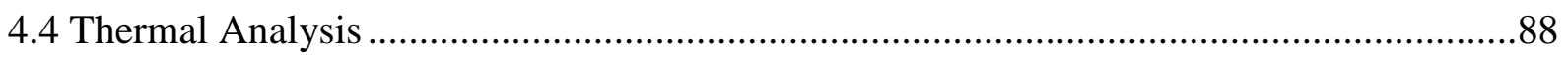

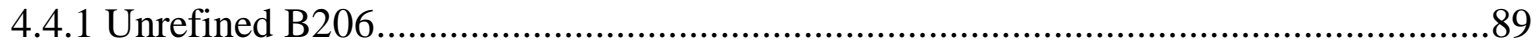

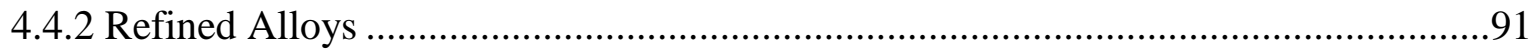

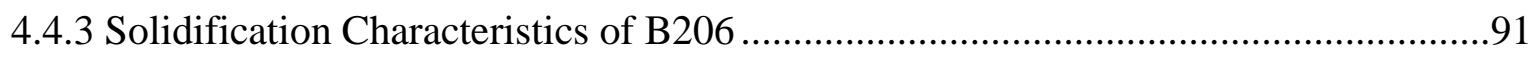

4.5 Neutron Diffraction Residual Strain Mapping ...............................................................96

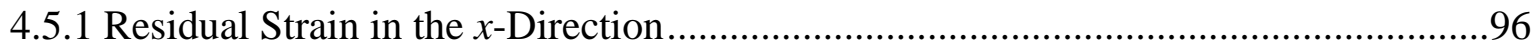

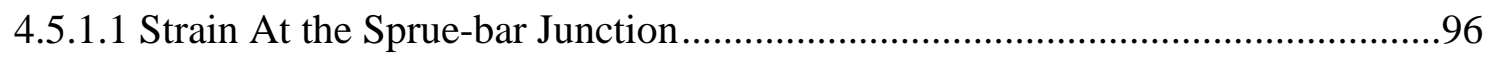

4.5.1.2 Strain Along the Horizontal Bar ....................................................................103

4.5.2 Residual Strain in the $y$-Direction....................................................................110

4.5.2.1 Residual Strain Along the Downsprue........................................................114

4.5.3 Residual Strain in the $z$-Direction ................................................................116

4.5.4 Residual Stress in B206 Castings ......................................................................121

4.5.4.1 Residual Stress in the $x$-Direction ...............................................................121

4.5.4.2 Residual Stress in the $y$ and $z$-Direction ..................................................122

Chapter 5 - In Situ Analysis of Hot Tearing in B206 ..................................................................128

5.1 Observation of Hot Tears on Casting Surfaces ..........................................................128

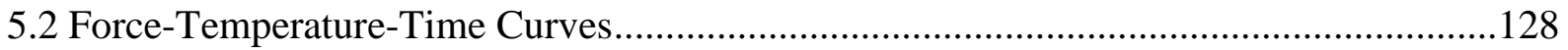

5.3 Casting Microstructure Analysis...................................................................................133

5.3.1 Grain Size and Morphology …………………….........................................133

5.3.2 General Alloy Microstructure ………………………………………………….....136

5.4 In Situ Neutron Diffraction Solidification Analysis .........................................................139

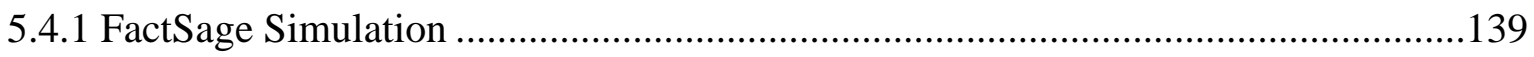

5.4.2 Growth of Primary Aluminum Phase …………………………………..............142

5.4.2.1 Grain Morphology of Samples........................................................................142

5.4.2.2 Primary Aluminum Peak Evolution...................................................................143

5.4.2.3 Primary Aluminum Phase Evolution ……………..............................................145

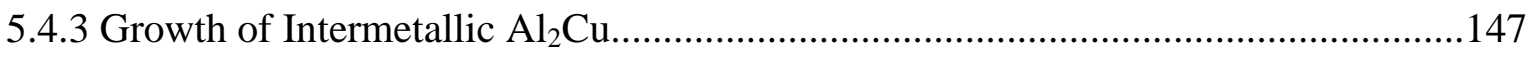

5.4.3.1 Intermetallic $\mathrm{Al}_{2} \mathrm{Cu}$ Peak Evolution...........................................................147

5.4.3.2 Intermetallic $\mathrm{Al}_{2} \mathrm{Cu}$ Phase Evolution .............................................................148 
5.4.4 The Role of Microstructure Development on Hot Tearing in B206 149

5.4.4.1 Onset Solid Fraction of Hot Tearing in B206 .149

5.4.4.2 Development of Microstructure in B206 ....................................................150

Chapter 6 - Conclusions........................................................................................155

Chapter 7 - Recommendations for Future Work ............................................................158

Appendix 1 - B206 Composition Analysis .......................................................................159

Appendix 2 - Micrographs at Hot Tear Region.................................................................161

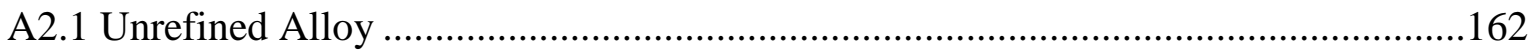

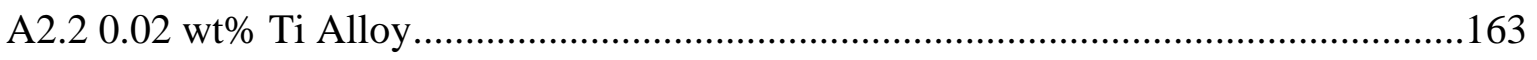

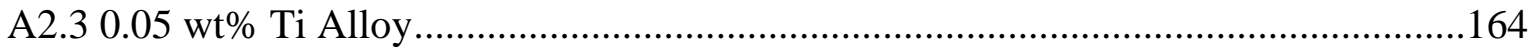

Appendix 3 - Cooling Curves and Thermal Analysis ........................................................165

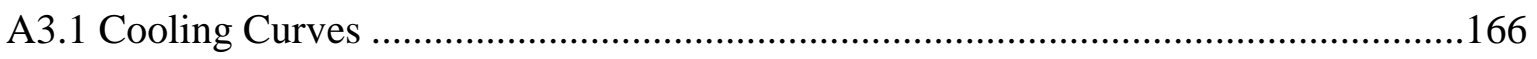

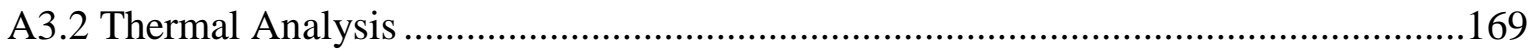

Appendix 4 - Residual Strain Profiles ....................................................................................170

A4.1 Residual Strain at the Sprue-Bar Junction ...................................................171

A4.2 Residual Strain Along the Horizontal Bar ..........................................................174

A4.3 Uncertainty in Residual Strain Calculations ....................................................175

Appendix 5 - Force-Temperature-Time Curves.......................................................................176

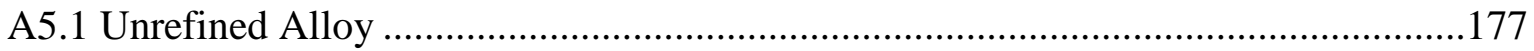

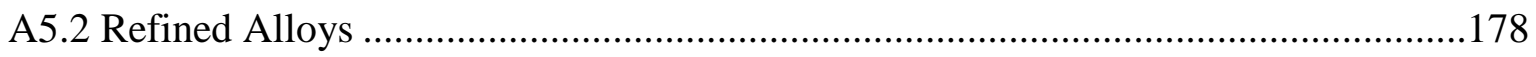

Appendix 6 - Peak Evolution of Primary Aluminum and Intermetallic $\mathrm{Al}_{2} \mathrm{Cu}$.................179

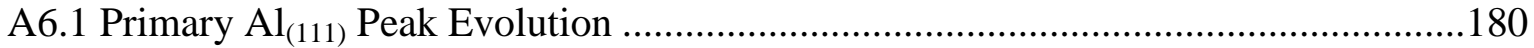

A6.2 Primary $\mathrm{Al}_{(200)}$ Peak Evolution .................................................................. 181

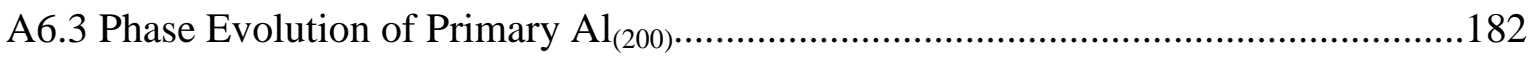

A6.4 Intermetallic $\mathrm{Al}_{2} \mathrm{Cu}_{(310)}$ Peak Evolution .........................................................183

Appendix 7 - List of Academic Achievements ......................................................................184

A7.1 Peer-Reviewed Journal Paper Publications.....................................................185

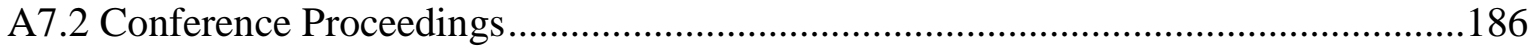

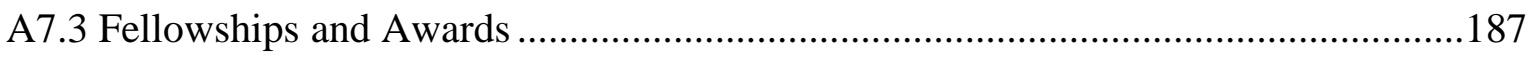

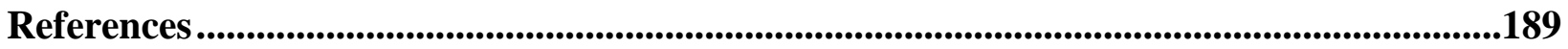




\section{List of Tables}

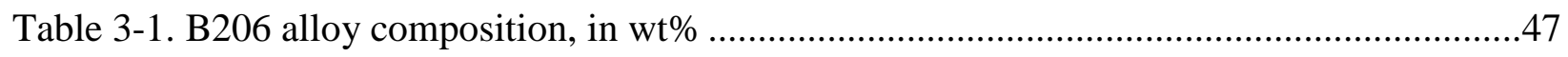

Table 3-2. Al-5Ti-1B master alloy composition, in wt\% ….................................................48

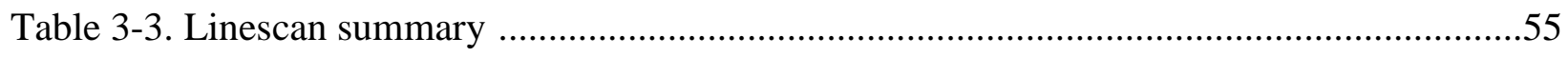

Table 3-4. Polishing procedure for microstructure analysis of samples .................................65

Table 4-1. Grain size measurements for B206 with various levels of Ti ................................71

Table 4-2. Thermal analysis data (by the sprue-bar junction) ..............................................93

Table 4-3. Differences in time at points of interest ..........................................................93

Table 4-4. Average dendrite/grain growth velocity in B206 ................................................95

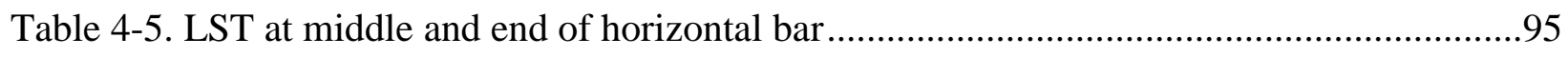

\section{Appendix Tables}

Table A.1-1. Composition of B206 used for ex situ casting experiments, in wt\% ....................160

Table A.1-2. Composition of B206 used for in situ casting experiments, in wt \% ....................160

Table A.1-3. Composition of binary Al-5 wt\% Cu alloy used for in situ neutron diffraction

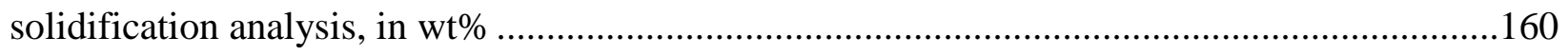

Table A.3-1. Thermal analysis data (by the middle of the horizontal bar) .............................169

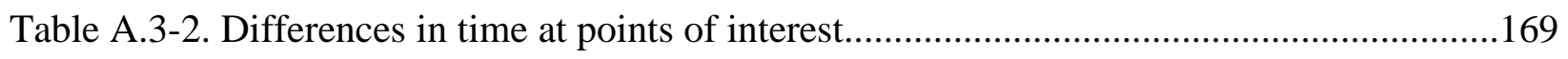

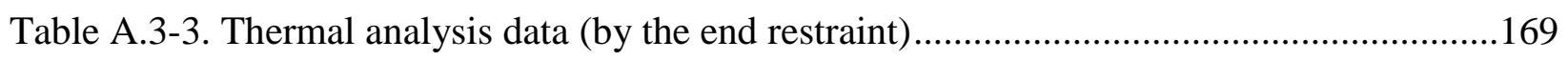

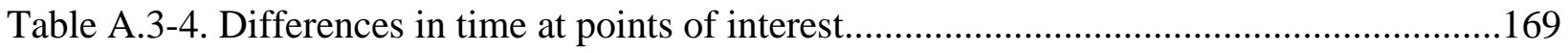




\section{List of Figures}

Figure 1-1. Dissertation overview...............................................................................4

Figure 2-1. Schematic diagram of the five feeding mechanisms during casting [1] ...................6

Figure 2-2. Graphical representation of the vulnerable time period, $t_{v}$ and the stress relief period, $t_{r}[21]$

Figure 2-3. Effect of solute content on hot tearing in a) Al-Cu alloys, b) Al-Si alloys, c) Al-Mg

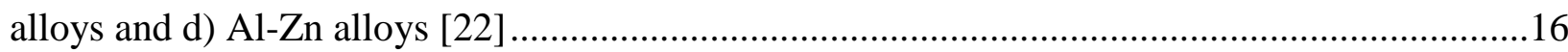

Figure 2-4. Lambda curves observed for Al-Mg alloys at varying superheats [23] ...................17

Figure 2-5. Plot showing increasing length of hot tears with increasing grain size for various Al alloys [22] .19

Figure 2-6. Ring mold test method and resulting ring casting [6] .......................................28

Figure 2-7. "Dog-bone" mold used to study hot tearing [6] .29

Figure 2-8. Modified “dog-bone” mold with samples of varying lengths (dimensions in mm) [6]

Figure 2-9. Modified "dog-bone” mold with varying sample diameters (dimensions in mm) [6]

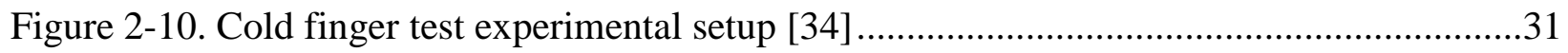

Figure 2-11. Hot tear apparatus developed by Instone et al [49] ..............................................33

Figure 2-12. Schematic diagram of Instone's hot tear test rig [49] .........................................33

Figure 2-13. The measured load at solidus temperature for a series of Al-Cu alloys recorded by

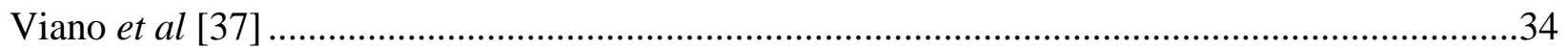

Figure 2-14. Instrumented CRC mold developed by Cao et al [51] ..........................................35

Figure 2-15. Load and cooling curve for an AZ91E Mg alloy [51] .........................................35

Figure 2-16. Hot tearing setup developed by Zhen et al [46] .................................................36

Figure 2-17. T-shaped mold equipped with moving wall and LVDT [39]................................37

Figure 2-18. Schematic illustration of a triple axis spectrometer [57] .......................................39

Figure 3-1. H-13 steel permanent mold (isometric view) ...................................................44

Figure 3-2. Dimensions of permanent mold (in $\mathrm{mm}$ ) ........................................................4

Figure 3-3. Cooper-Chapman pneumatic sand core-making machine with mounted mold ..........45

Figure 3-4. Location of thermocouples along casting cavity .............................................47

Figure 3-5. Triple axis spectrometer in Chalk River [57] .................................................49 
Figure 3-6. Sample setup for neutron diffraction strain mapping experiments for the a) $\mathrm{x}$ direction scan, b) y-direction scan and c) z-direction scan ...............................................51

Figure 3-7. Coordinate axes of spectrometer in relation to casting geometry [57] .....................52

Figure 3-8. Neutron beam sampling volume [57] .............................................................52

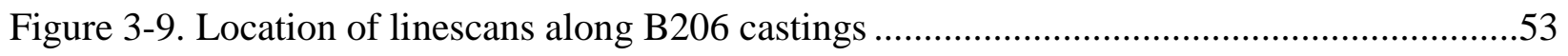

Figure 3-10. Length of linescans across horizontal bar .....................................................54

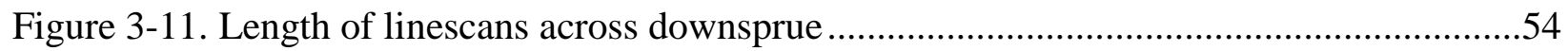

Figure 3-12. Location of extracted stress-free samples [57] .............................................56

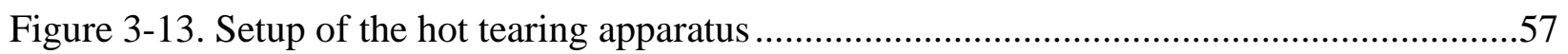

Figure $3-14$. Location of thermocouples across casting ….................................................58

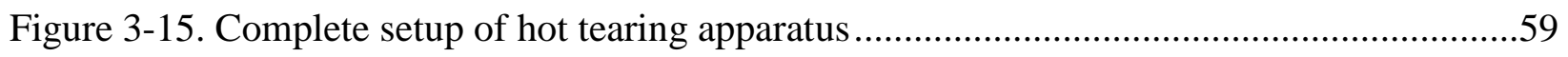

Figure 3-16. An example force-temperature-time curve for B206 .........................................60

Figure 3-17. Dimensions of solidification sample (dimensions in $\mathrm{mm}$ )..................................62

Figure 3-18. Experimental setup for neutron diffraction solidification analysis ......................63

Figure 3-19. Graphite crucible (dimensions in $\mathrm{mm}$ ) ...........................................................64

Figure 4-1. Hot tearing along the middle of the horizontal bar of the unrefined B206 alloy .......67

Figure 4-2. Close up of a) backside and b) front of sprue-bar junction of the unrefined B206 alloy

Figure 4-3. Image of casting of 0.02 wt\% Ti B206 alloy ................................................68

Figure 4-4. Examination of a) backside and b) front of sprue-bar junction of the $0.02 \mathrm{wt} \% \mathrm{Ti}$ B206 alloy revealing c) the presence of a hot tear .............................................................69

Figure 4-5. Image of casting of 0.05 wt\% Ti B206 alloy ..................................................69

Figure 4-6. Close up of a) backside and b) front of sprue-bar junction of the $0.05 \mathrm{wt} \% \mathrm{Ti}$ B206

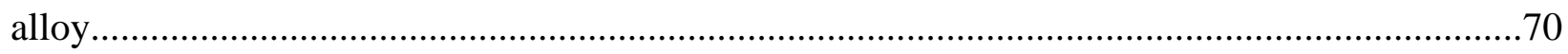

Figure 4-7. Binary Al-Cu phase diagram (Al-rich side) [67] ................................................74

Figure 4-8. Grain morphology in unrefined B206 alloy (100x) ..........................................75

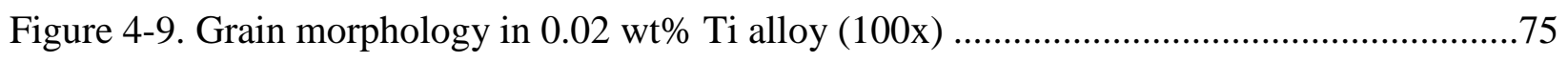

Figure 4-10. Grain morphology in $0.05 \mathrm{wt} \%$ Ti alloy $(100 \mathrm{x})$............................................75

Figure 4-11. Morphology of $\mathrm{Al}_{2} \mathrm{Cu}$ intermetallic in unrefined B206 alloy ..............................76

Figure 4-12. Morphology of $\mathrm{Al}_{2} \mathrm{Cu}$ intermetallic in $0.02 \mathrm{wt} \% \mathrm{Ti}$ alloy ..................................76

Figure 4-13. Morphology of $\mathrm{Al}_{2} \mathrm{Cu}$ intermetallic in $0.05 \mathrm{wt} \% \mathrm{Ti}$ alloy ..................................76

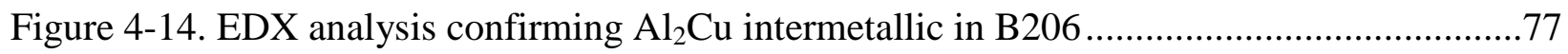


Figure 4-15. EDX analysis confirming Fe and Mn-bearing intermetallics in B206 .77

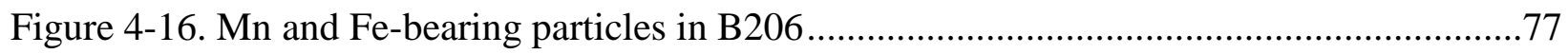

Figure 4-17. EDX analysis confirming the presence of $\mathrm{Ti}$ in the middle of Al grains .................78

Figure 4-18. Location of extracted samples along a) front view and b) rear view of sprue-bar

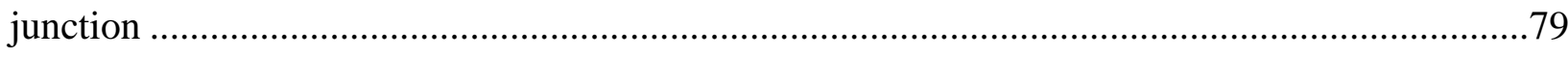

Figure 4-19. Sprue-bar junction in unrefined B206 depicting hot tears .................................80

Figure 4-20. Propagation of cracks through interdendritic regions in unrefined B206 ...............80

Figure 4-21. Dendritic morphology along hot tear fracture surface in unrefined B206 ..............81

Figure 4-22. EDX analysis confirming $\mathrm{Al}_{2} \mathrm{Cu}$ along hot tear fracture surface of unrefined B206

Figure 4-23. Presence of hot tear along sprue-bar junction in $0.02 \mathrm{wt} \% \mathrm{Ti}$ alloy .......................83

Figure 4-24. Dendritic hot tear fracture surface in 0.02 wt\% Ti alloy.......................................84

Figure 4-25. Evidence of grain deformation along sprue-bar junction in $0.05 \mathrm{wt} \%$ Ti alloy........85

Figure 4-26. A closer view of the deformed grains in $0.05 \mathrm{wt} \% \mathrm{Ti}$ alloy .................................85

Figure 4-27. Percent area porosity measurements for B206 with various levels of Ti.................86

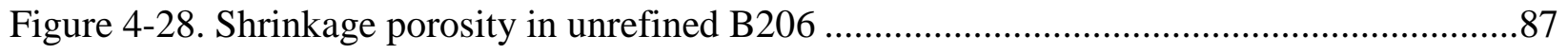

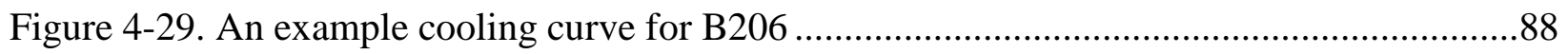

Figure 4-30. Cooling curve and its first derivative for the unrefined B206 alloy ......................90

Figure 4-31. Evidence of undercooling in first derivative curve of unrefined B206..................90

Figure 4-32. Cooling curve and its first derivative for the $0.02 \mathrm{wt} \% \mathrm{Ti}$ alloy...........................92

Figure 4-33. Cooling curve and its first derivative for the $0.05 \mathrm{wt} \% \mathrm{Ti}$ alloy...........................92

Figure 4-34. Evidence of no undercooling in first derivative curve of the $0.02 \mathrm{wt} \%$ Ti alloy ......93

Figure 4-35. Profiles of $\varepsilon_{x}$ at the sprue-bar junction along the a) top edge; b) bottom edge and c) centreline of the unrefined alloy's horizontal bar for the (111) reflection 100

Figure 4-36. Profiles of $\varepsilon_{x}$ at the sprue-bar junction along the a) top edge; b) bottom edge and c) centreline of the $0.02 \mathrm{wt} \%$ Ti alloy's horizontal bar for the (111) reflection 101

Figure 4-37. Profiles of $\varepsilon_{x}$ at the sprue-bar junction along the a) top edge; b) bottom edge and c) centreline of the $0.05 \mathrm{wt} \%$ Ti alloy's horizontal bar for the (111) reflection 103

Figure 4-38. Profiles of $\varepsilon_{x}$ along the a) top edge and b) bottom edge of the horizontal bar for the (311) reflection of the unrefined alloy .105

Figure 4-39. Profiles of $\varepsilon_{x}$ along the a) top edge and b) bottom edge of the horizontal bar for the (311) reflection of the $0.02 \mathrm{wt} \% \mathrm{Ti}$ alloy .106 
Figure 4-40. Profiles of $\varepsilon_{x}$ along the (a) top edge and (b) bottom edge of the horizontal bar for the (311) reflection of the $0.05 \mathrm{wt} \%$ Ti alloy

Figure 4-41. Unrestrained B206 casting illustrating a) a front view of the hot tear (while inside the mold) and b) a rear view of the hot tear along the horizontal bar. .108

Figure 4-42. Variance in $x$-direction strain magnitude along the horizontal bar for the three B206 castings

Figure 4-43. Profiles of $\varepsilon_{y}$ along the a) top edge and b) bottom edge of the horizontal bar for the (311) reflection of the unrefined alloy .111

Figure 4-44. Profiles of $\varepsilon_{y}$ along the a) top edge and b) bottom edge of the horizontal bar for the (311) reflection of the $0.02 \mathrm{wt} \% \mathrm{Ti}$ alloy

Figure 4-45. Profiles of $\varepsilon_{y}$ along the a) top edge and b) bottom edge of the horizontal bar for the (311) reflection of the $0.05 \mathrm{wt} \%$ Ti alloy

Figure 4-46. Variance in $y$-direction strain magnitude along the horizontal bar for the three B206 castings.

Figure 4-47. Profiles of $\varepsilon_{y}$ along the downsprue of the a) unrefined B206; b) $0.02 \mathrm{wt} \% \mathrm{Ti}$ and c) $0.05 \mathrm{wt} \% \mathrm{Ti}$ alloys for the (111) reflection...

Figure 4-48. Profiles of $\varepsilon_{z}$ along the a) top edge and b) bottom edge of the horizontal bar for the (311) reflection of the unrefined alloy

Figure 4-49. Profiles of $\varepsilon_{z}$ along the a) top edge and b) bottom edge of the horizontal bar for the (311) reflection of the $0.02 \mathrm{wt} \% \mathrm{Ti}$ alloy .118

Figure 4-50. Profiles of $\varepsilon_{z}$ along the a) top edge and b) bottom edge of the horizontal bar for the (311) reflection of the $0.05 \mathrm{wt} \%$ Ti alloy

Figure 4-51. Variance in $z$-direction strain magnitude along the horizontal bar for the three B206 castings.

Figure 4-52. Profiles of $\sigma_{x}$ along the a) top edge and b) bottom edge of the horizontal bar for the (311) reflection of the unrefined B206 alloy ....

Figure 4-53. Profiles of $\sigma_{x}$ along the a) top edge and b) bottom edge of the horizontal bar for the (311) reflection of the $0.02 \mathrm{wt} \% \mathrm{Ti}$ and $0.05 \mathrm{wt} \% \mathrm{Ti}$ alloys

Figure 4-54. Profiles of $\sigma_{y}$ and $\sigma_{z}$ along the a) top edge and b) bottom edge of the horizontal bar for the (311) reflection of the unrefined B206 alloy.

Figure 4-55. Profiles of $\sigma_{y}$ and $\sigma_{z}$ along the a) top edge and b) bottom edge of the horizontal bar for the (311) reflection of the $0.02 \mathrm{wt} \% \mathrm{Ti}$ alloy.

Figure 4-56. Profiles of $\sigma_{y}$ and $\sigma_{z}$ along the a) top edge and b) bottom edge of the horizontal bar for the (311) reflection of the $0.05 \mathrm{wt} \% \mathrm{Ti}$ alloy. 
Figure 5-1. The casting surfaces of a) unrefined B206 at $250{ }^{\circ} \mathrm{C}$ mold temperature; b) unrefined B206 at $325{ }^{\circ} \mathrm{C}$ mold temperature; c) unrefined B206 at $400{ }^{\circ} \mathrm{C}$ mold temperature; d) 0.02 wt $\%$ Ti alloy $400{ }^{\circ} \mathrm{C}$ mold temperature and e) $0.05 \mathrm{wt} \%$ Ti alloy at $400{ }^{\circ} \mathrm{C}$ mold temperature 129

Figure 5-2. Force-temperature-time curves for unrefined B206 at a) $250{ }^{\circ} \mathrm{C}$; b) $325{ }^{\circ} \mathrm{C}$ and c) $400{ }^{\circ} \mathrm{C}$ mold temperatures

Figure 5-3. Force-temperature-time curves for a) $0.02 \mathrm{wt} \%$ Ti alloy and b) $0.05 \mathrm{wt} \%$ Ti alloy.132

Figure 5-4. Effect of mold temperature and Ti level on grain size in B206

Figure 5-5. Grain morphology of unrefined B206 at mold temperatures of a) $250{ }^{\circ} \mathrm{C}$; b) $325^{\circ} \mathrm{C}$

and c) $400{ }^{\circ} \mathrm{C}$. .134

Figure 5-6. Grain morphology of the a) unrefined B206 alloy; b) $0.02 \mathrm{wt} \%$ Ti alloy and c) 0.05 wt $\%$ Ti alloy.....

Figure 5-7. Effect of a) $250{ }^{\circ} \mathrm{C}$; b) $325^{\circ} \mathrm{C}$ and c) $400{ }^{\circ} \mathrm{C}$ mold temperature on $\mathrm{Al}_{2} \mathrm{Cu}$ in unrefined B206. 136

Figure 5-8. EDX analysis confirming the $\mathrm{Al}_{2} \mathrm{Cu}$ intermetallic ..... .137

Figure 5-9. Effect of mold temperature on percent area of $\mathrm{Al}_{2} \mathrm{Cu}$. .137

Figure 5-10. Effect of $\mathrm{Ti}$ on $\mathrm{Al}_{2} \mathrm{Cu}$ intermetallic in a) $0.02 \mathrm{wt} \% \mathrm{Ti}$ alloy and b) $0.05 \mathrm{wt} \% \mathrm{Ti}$ alloy .138

Figure 5-11. Effect of $\mathrm{Ti}$ on percent area of $\mathrm{Al}_{2} \mathrm{Cu}$ .138

Figure 5-12. FactSage calculation of Al-5 wt\% $\mathrm{Cu}$ alloy for equilibrium solidification .140

Figure 5-13. FactSage calculation of Al-5 wt\% Cu alloy for non-equilibrium solidification .....141

Figure 5-14. Grain morphology of a) unrefined; b) $0.02 \mathrm{wt} \% \mathrm{Ti}$ and c) $0.05 \mathrm{wt} \% \mathrm{Ti}$ binary alloy prior to neutron diffraction solidification analysis.

Figure 5-15. Grain morphology of a) unrefined; b) $0.02 \mathrm{wt} \% \mathrm{Ti}$ and c) $0.05 \mathrm{wt} \%$ Ti binary alloy following neutron diffraction solidification analysis .143

Figure 5-16. Peak evolution of the $\alpha-\mathrm{Al}_{(111)}$ phase .144

Figure 5-17. Phase evolution of $\alpha-\mathrm{Al}_{(111)}$ solid solution in the unrefined (UNR), $0.02 \mathrm{wt} \% \mathrm{Ti}$ and $0.05 \mathrm{wt} \%$ Ti binary Al-5 wt\% Cu alloys .146

Figure 5-18. Peak evolution of the $\mathrm{Al}_{2} \mathrm{Cu}_{(310)}$ intermetallic phase for the $0.05 \mathrm{wt} \%$ Ti alloy .....148 Figure 5-19. Phase evolution of intermetallic $\mathrm{Al}_{2} \mathrm{Cu}_{(310)}$ in the unrefined (UNR), $0.02 \mathrm{wt} \% \mathrm{Ti}$ and $0.05 \mathrm{wt} \%$ Ti binary Al-5 wt\% Cu alloys .149

Figure 5-20. Combined growth profiles of $\alpha-\mathrm{Al}$ and $\mathrm{Al}_{2} \mathrm{Cu}$ in the unrefined alloy.... .151

Figure 5-21. The presence of blocky $\mathrm{Al}_{2} \mathrm{Cu}$ in the unrefined alloy at a) $50 \mathrm{x}$ and b) $200 \mathrm{x}$ magnification . .151 
Figure 5-22. Combined growth profiles of $\alpha-\mathrm{Al}$ and $\mathrm{Al}_{2} \mathrm{Cu}$ in the $0.02 \mathrm{wt} \% \mathrm{Ti}$ alloy .153

Figure 5-23. Combined growth profiles of $\alpha-\mathrm{Al}$ and $\mathrm{Al}_{2} \mathrm{Cu}$ in the $0.05 \mathrm{wt} \% \mathrm{Ti}$ alloy .153

Figure 5-24. The presence of blocky and eutectic $\mathrm{Al}_{2} \mathrm{Cu}$ in the $0.05 \mathrm{wt} \%$ Ti alloy at a) 100x and

b) 500x magnification .154

\section{Appendix Figures}

Figure A.2-1. Hot tears along a) Sample \#1; b) Sample \#3; c) Sample \#2 and d) Sample \#4 at the sprue-bar junction of the unrefined B206 alloy .... .162

Figure A.2-2. Hot tears along a) Sample \#1; b) Sample \#3; c) Sample \#2 and d) Sample \#4 at the sprue-bar junction of the $0.02 \mathrm{wt} \%$ Ti alloy .163

Figure A.2-3. Hot tears along a) Sample \#1; b) Sample \#3; c) Sample \#2 and d) Sample \#4 at the sprue-bar junction of the $0.05 \mathrm{wt} \% \mathrm{Ti}$ alloy .164

Figure A.3-1. Cooling curve and its first derivative for the unrefined B206 alloy (by the middle of the horizontal bar). .166

Figure A.3-2. Cooling curve and its first derivative for the unrefined B206 alloy (by the end restraint)

Figure A.3-3. Cooling curve and its first derivative for the $0.02 \mathrm{wt} \% \mathrm{Ti}$ alloy (by the middle of the horizontal bar).....

Figure A.3-4. Cooling curve and its first derivative for the $0.02 \mathrm{wt} \% \mathrm{Ti}$ alloy (by the end restraint)

Figure A.3-5. Cooling curve and its first derivative for the $0.05 \mathrm{wt} \% \mathrm{Ti}$ alloy (by the middle of the horizontal bar). .168

Figure A.3-6. Cooling curve and its first derivative for the $0.05 \mathrm{wt} \%$ Ti alloy (by the end restraint)

Figure A.4-1. Profiles of $\varepsilon_{\mathrm{x}}$ at the sprue-bar junction along the a) top edge and b) bottom edge of the unrefined B206 alloy's horizontal bar for the (311) reflection....

Figure A.4-2. Profiles of $\varepsilon_{\mathrm{x}}$ at the sprue-bar junction along the a) top edge and b) bottom edge of the $0.02 \mathrm{wt} \%$ Ti alloy's horizontal bar for the (311) reflection

Figure A.4-3. Profiles of $\varepsilon_{\mathrm{x}}$ at the sprue-bar junction along the a) top edge and b) bottom edge of the $0.05 \mathrm{wt} \%$ Ti alloy's horizontal bar for the (311) reflection.....

Figure A.4-4. Profiles of $\varepsilon_{\mathrm{x}}$ along the a) top edge and b) bottom edge of the horizontal bar for the (111) reflection of the unrefined B206 alloy ..... 
Figure A.5-1. Force-temperature-time curves for unrefined B206 at a) $250{ }^{\circ} \mathrm{C}$; b) $325{ }^{\circ} \mathrm{C}$ and c) $400{ }^{\circ} \mathrm{C}$ mold temperatures

Figure A.5-2. Force-temperature-time curves for a) $0.02 \mathrm{wt} \% \mathrm{Ti}$ alloy and b) $0.05 \mathrm{wt} \% \mathrm{Ti}$

alloy 178

Figure A.6-1. Peak evolution of $\alpha-\mathrm{Al}_{(111)}$ phase in unrefined binary $\mathrm{Al}-5 \mathrm{wt} \% \mathrm{Cu}$ alloy ..........180

Figure A.6-2. Peak evolution of $\alpha-\mathrm{Al}_{(111)}$ phase in $0.02 \mathrm{wt} \%$ Ti binary Al-5 wt\% Cu alloy.......180

Figure A.6-3. Peak evolution of $\alpha-\mathrm{Al}_{(200)}$ phase in $0.02 \mathrm{wt} \%$ Ti binary Al-5 wt\% Cu alloy.......181

Figure A.6-4. Peak evolution of $\alpha-\mathrm{Al}_{(200)}$ phase in $0.05 \mathrm{wt} \%$ Ti binary Al-5 wt\% Cu alloy.......181

Figure A.6-5. Phase evolution of $\alpha-\mathrm{Al}_{(200)}$ solid solution in the refined binary $\mathrm{Al}-5 \mathrm{wt} \% \mathrm{Cu}$ alloys .182

Figure A.6-6. Peak evolution of $\mathrm{Al}_{2} \mathrm{Cu}_{(310)}$ phase in unrefined binary Al-5 wt $\% \mathrm{Cu}$ alloy .........183

Figure A.6-7. Peak evolution of $\mathrm{Al}_{2} \mathrm{Cu}_{(310)}$ phase in $0.02 \mathrm{wt} \%$ Ti binary Al-5 wt\% Cu alloy ....183 


\section{List of Appendices}

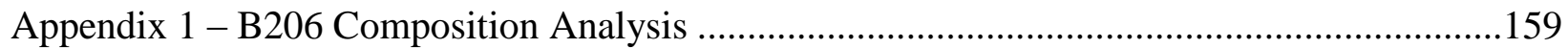

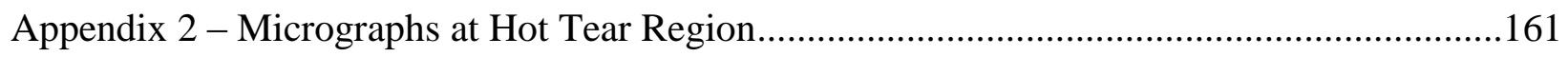

A2.1 Unrefined Alloy …………………………………….........................................162

A2.2 0.02 wt\% Ti Alloy ……………………………………..................................163

A2.3 0.05 wt\% Ti Alloy .............................................................................................164

Appendix 3 - Cooling Curves and Thermal Analysis ...............................................................165

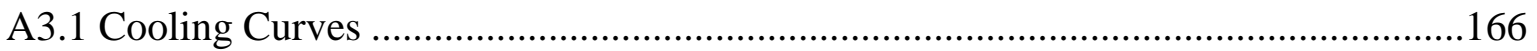

A3.2 Thermal Analysis ...............................................................................................169

Appendix 4 - Residual Strain Profiles...................................................................................170

A4.1 Residual Strain at the Sprue-Bar Junction ..........................................................171

A4.2 Residual Strain Along the Horizontal Bar ...........................................................174

A4.3 Uncertainty in Residual Strain Calculations ............................................................175

Appendix 5 - Force-Temperature-Time Curves ......................................................................176

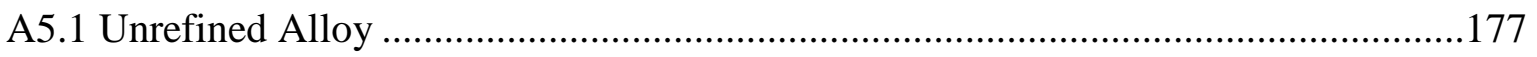

A5.2 Refined Alloys ...................................................................................................178

Appendix 6 - Peak Evolution of Primary Aluminum and Intermetallic $\mathrm{Al}_{2} \mathrm{Cu}$..........................179

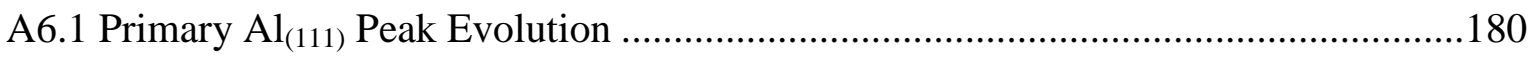

A6.2 Primary $\mathrm{Al}_{(200)}$ Peak Evolution ........................................................................181

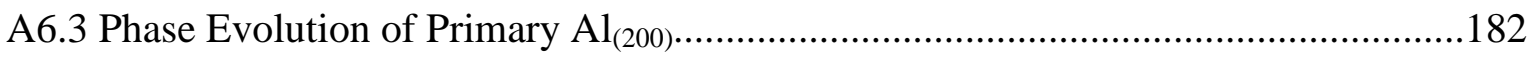

A6.4 Intermetallic $\mathrm{Al}_{2} \mathrm{Cu}_{(310)}$ Peak Evolution .............................................................183

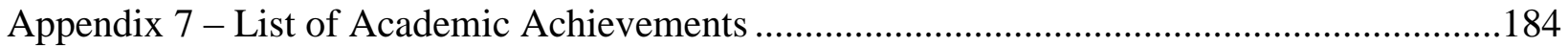

A7.1 Peer-Reviewed Journal Paper Publications..........................................................185

A7.2 Conference Proceedings..................................................................................186

A7.3 Fellowships and Awards ..................................................................................187 


\section{Nomenclature}

\section{Greek}

$\alpha \quad$ Surface tension in liquid

$\alpha \quad$ Primary

$\varepsilon \quad$ Strain

$\theta \quad$ Bragg angle

$\sigma \quad$ Stress

$\lambda \quad$ Wavelength

$v \quad$ Poisson's ratio

\section{English}

$B$

E

$l_{0}$

$n$

$P$

$T_{C}$

$T_{G}$

$T_{N}$

$T_{S}$

$t_{C}$

$t_{G}$

$t_{N}$

$t_{S}$

$t_{r}$

$t_{v}$

\section{Elements}

Liquid film thickness

$\mathrm{cm}$

Young's modulus of elasticity

$\mathrm{MPa}$

Characteristic length

$\mu \mathrm{m}$

Order of diffraction

Molecular adhesion force

$\mathrm{N}$

Coherency temperature

${ }^{\circ} \mathrm{C}$

Growth temperature

${ }^{\circ} \mathrm{C}$

Nucleation temperature

${ }^{\circ} \mathrm{C}$

End of solidification temperature

${ }^{\circ} \mathrm{C}$

Time at coherency temperature

S

Time at growth temperature

S

Time at nucleation temperature

S

Time at end of solidification

S

Time available for stress relief

$\mathrm{S}$

Vulnerable time interval
$\mathrm{Al}$
Aluminum
B
Boron 


$\begin{array}{ll}\mathrm{BN} & \text { Boron-nitride } \\ \mathrm{Cu} & \text { Copper } \\ \mathrm{RE} & \text { Rare earth } \\ \mathrm{Gd} & \text { Gadolinium } \\ \mathrm{Ge} & \text { Germanium } \\ \mathrm{Fe} & \text { Iron } \\ \mathrm{Mg} & \text { Magnesium } \\ \mathrm{Mn} & \text { Manganese } \\ \mathrm{Si} & \text { Silicon } \\ \mathrm{Sn} & \text { Tin } \\ \mathrm{Ti} & \text { Titanium } \\ \mathrm{Y} & \text { Yttrium } \\ \mathrm{Zn} & \text { Zinc }\end{array}$

\section{Acronyms}

BSE Backscattered electron

CR Cooling rate

CRC Constrained-rod-casting

CSC Crack Susceptibility Coefficient

DC Direct chill

EDX Energy dispersive X-ray

FR Freezing range

LST Local solidification time

OM Optical microscopy

PID Proportional-integral-derivative

SEM Scanning electron microscopy

UNR Unrefined 


\section{Chapter 1 - Introduction}

There is a growing interest in aluminum-copper $(\mathrm{Al}-\mathrm{Cu})$ alloys for use in automotive and aerospace industries. These alloys are high in strength and ductility and therefore, can be incorporated in a variety of components. One such alloy with potential for use in automotive control arms and suspension knuckles is the B206 alloy. B206 has Cu concentration ranging from $4.2-5.0 \mathrm{wt} \%$, which makes it precipitation hardenable and capable of possessing enhanced mechanical properties. In fact, in the heat-treated state, this alloy has mechanical properties comparable to those of ductile iron at one-third the density. In turn, replacement of ductile iron with B206 can result in significant lightweighting of vehicles. Despite its advantages, however, the limiting factor of the B206 alloy is its high susceptibility to hot tearing during casting.

Hot tears are cracks forming on a semi-solid alloy prior to its complete solidification. Generally, there are two main categories of factors responsible for hot tearing in castings: metallurgical factors and mechanical factors. The metallurgical aspects, such as alloy grain size and morphology of intermetallic phases, influence both the semi-solid mechanical properties (i.e. strength and ductility) of the alloy as well as the ability of the liquid metal to feed the solidifying alloy. Such factors are directly affected by the alloy's solidification characteristics. The mechanical factors, on the other hand, are due to the casting deformation caused by thermal contraction within a rigid steel mold. The rigidity of the mold restricts contraction of the casting during cooling, thereby inducing tensile strain and stress and promoting the formation of hot tears in the casting. The major hindrance in hot tearing analysis is the absence of a method to quantitatively measure the stress and strain causing hot tears in castings. In the case of the remaining factors (i.e. solidification and microstructure), numerous techniques are readily available for characterization.

Casting solidification can be characterized by thermal analysis whereby thermocouple probes are placed in a casting. This technique enables the determination of the casting's cooling rate, freezing range and solidification time. Further, supplemental data such as the coherency temperature, $T_{C}$, can be detected and used to gain an understanding of the casting's feeding behaviour during solidification. 
In the case of casting deformation, the modern approach uses computer simulation to predict the evolution of casting microstructure and stress during solidification. However, such analyses are elusive in that many factors (e.g. casting geometry, material properties, etc) are simplified with suitable assumptions. As such, the results obtained (e.g. mechanical properties, onset of hot tearing) are not representative of actual casting experiments.

The purpose of this research was to develop a unique approach to characterize the fundamental mechanisms of hot tearing in B206 aluminum alloy castings. Elastic residual strain and stress were measured using neutron diffraction and the results were directly related to the hot tearing characteristics of B206. The alloy grain morphology and microstructure was characterized using optical and scanning electron microscopy. Thermal analysis was carried out to gain an understanding of the solidification characteristics and feeding behaviour of B206. Further, in situ analysis techniques were used to identify the onset temperature and solid fraction of hot tearing in B206, as well as characterize the development of microstructure during solidification of B206. Thus, a direct correlation of microstructure, solidification and residual strain and stress was developed and enabled an improved understanding of the mechanisms of hot tearing in B206.

This dissertation has been structured as follows.

Chapter 2 presents a concise review of the literature available on alloy solidification, the theories of hot tearing, the factors affecting hot tearing, including alloy composition, grain size and processing parameters, and finally, the various techniques used to characterize hot tearing susceptibility.

Chapter 3 describes the experimental methodology undertaken in this research, including melting and casting experiments, neutron diffraction measurements and microscopic evaluation.

Chapter 4 presents the results of ex situ casting experiments, including thermal analysis, microscopy and the measurements of residual strain and stress. A discussion on the significance of such results in relation to hot tearing is also presented. 
Chapter 5 presents the results of the in situ casting experiments, including the determination of the onset temperature and solid fraction of hot tearing and the results of in situ neutron diffraction solidification analysis.

Chapter 6 summarizes the conclusions obtained from this research.

Chapter 7 offers a list of recommendations for future research.

The flowchart in Figure 1-1 depicts an overview of the research presented in this dissertation. The ex situ portion of the research consisted of permanent mold casting, microstructure and thermal analysis and finally ex situ measurement of residual strain in the castings. Such aspects were directly related to hot tearing severity in B206. A similar methodology was carried out for the in situ portion of the dissertation. However, in this case, the permanent mold enabled the determination of the onset temperature of hot tearing in B206, while neutron diffraction was used to establish the onset solid fraction of hot tearing and to gain an understanding of the in situ development of microstructure during solidification of B206. 


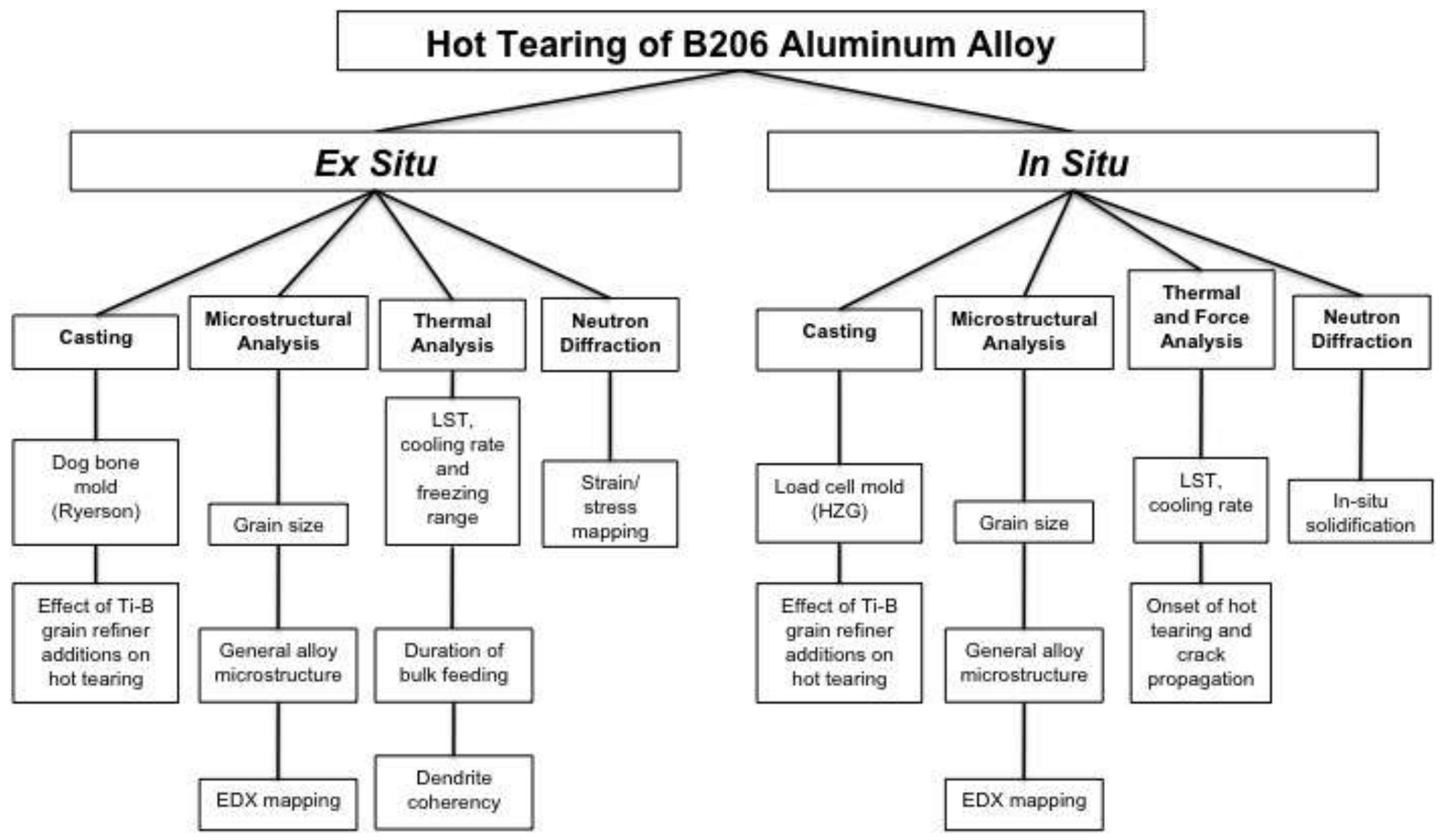

Figure 1-1. Dissertation overview. 


\section{Chapter 2-Literature Review}

The chapter begins with a description of alloy solidification during casting with emphasis on the feeding mechanisms. A review on hot tearing follows, including a summary of hot tearing theories and a description on the effect of alloy composition, grain size and morphology and processing parameters on hot tearing. Finally, the chapter concludes with a description of the common tests used to characterize hot tearing susceptibility.

\subsection{Alloy Solidification}

During the solidification of a casting, most alloys freeze over a temperature range and solidification occurs gradually with decreasing temperature. As the temperature decreases, the developing solid fraction leads to a continuously changing mush microstructure and presents increasing difficulties for the passage of feeding liquid [1,2]. Further, the freezing liquid continuously contracts to form solid, resulting in decreasing pressure in the liquid and generating a pressure difference between the inside and outside of the casting. This internal pressure might decrease enough to become negative, as a hydrostratic tension, and is undesirable in casting, as it is the driving force for the initiation and development of defects including porosity and hot tearing [1]. As a result, the adequate feeding of liquid during solidification is vital in limiting the probability of defect formation in castings. Campbell [1] identified five feeding mechanisms (i.e. liquid feeding, mass feeding, interdendritic feeding, burst feeding and solid feeding) that take place during alloy solidification. Figure 2-1 shows a schematic of the five feeding mechanisms during casting. The mechanisms are described hereunder in the order in which they generally occur.

Liquid feeding is generally the first feeding mechanism to take place during solidification. At the beginning of solidification, the liquid flows freely before any solid formation or through large channels between solid (or partially solid) regions [3]. The driving forces for liquid feeding are the accommodation of liquid contraction as the temperature decreases and the pressure differential resulting from distant solidification shrinkage. As solidification progresses and the alloy temperature cools, nucleation of solid particles takes place. Such solid particles, or nuclei, 
are responsible for the growth of grains in alloys. Each nucleant develops into a grain and therefore the number of nuclei is important in determining the final grain size of the casting. The more nuclei present in the melt, the finer the grain size [4]. Once nuclei exist in the melt, shrinkage (i.e. pressure differential) induced flow occurs by what is termed 'mass feeding' [2]. At this stage, crystals are suspended in the flowing melt. Both liquid and mass feeding are efficient at feeding the solidification shrinkage since there is no barrier to the liquid movement. Further, the casting's solid structure has very little coherence and therefore is no stress accumulation in the casting at this stage. Thus, the casting is not prone to porosity or hot tearing during this stage of solidification.

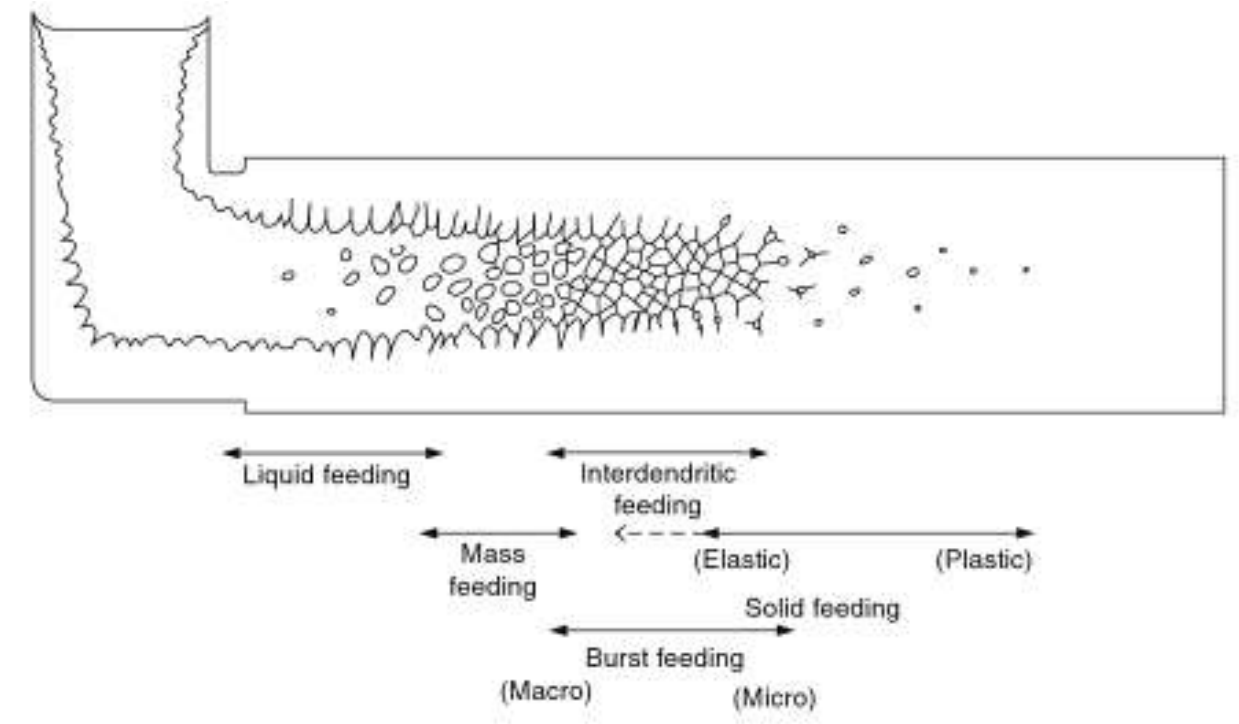

Figure 2-1. Schematic diagram of the five feeding mechanisms during casting [1].

As solidification progresses, growing dendrite arms begin to impinge on one another and eventually form a solid network. The solid fraction at which this occurs is called the dendrite coherency point and typically ranges between 0.25 and 0.6 [5,6]. At this stage of solidification, stresses arise in the network as the dendrite arms begin to mechanically interact with one another. Further, the solidifying mass loses the characteristics of a viscous liquid and becomes a semi-solid paste [3]. As a result, the mobility of liquid metal is reduced and the remaining liquid is limited to regions through the dendrite network. Thus, the dendrite coherency point marks the 
onset of interdendritic feeding, although some mass feeding can still continue due to small-scale movement of dendrites [2].

With increasing solid fraction the dendrite network becomes more closely packed leading to smaller interdendritic feeding channels. In turn, this makes it increasingly difficult for the remaining liquid to flow and accommodate solidification shrinkage. If a shrinking region in a casting is not fed by interdendritic flow, a hydrostatic tension develops in the liquid and imposes an increasing stress on the solid network [1]. The increasing stress at this region may exceed the strength of the solid network and cause the network to collapse or "burst". This feeding mechanism is hence termed burst feeding. During burst feeding, the broken or "burst" portions of dendrite arms are suspended in the melt, similar to that which occurs during mass feeding. Burst feeding occurs at select regions within a casting (i.e. where stresses are high enough to collapse the solid network) and takes place simultaneously with interdendritic feeding. Regions along the casting may also exist whereby either the imposed stress on the solid network is not sufficient to yield the barrier to interdendritic flow, or the strength of the barrier is substantial such that it may not yield. In this case, the stress will continuously increase and any release of stress will correspond to the creation and growth of a pore [1]. Thus, burst feeding is a desirable mechanism that enhances the permeability of the solidifying network and limits the formation of defects.

During the final stage of solidification, the dendrite network develops into a rigid solid skeleton and regions of liquid become isolated. At this stage, the feeding mechanism that takes place was identified as solid feeding. Pools of liquid remain in pockets between dendrite arms due to the high enrichment of solute at these regions [4]. As solidification progresses an increasing number of dendrite arms and dendrites coalesce resulting in more liquid regions becoming isolated [2]. Hence, the permeability of the solid network becomes too low for liquid to flow through and further thermal contraction will cause pores to grow and hot tears to form [6].

\section{Section Summary}

The feeding mechanisms during solidification of casting alloys were described. It was shown that the permeability of the solidifying network is strongly dependent on the continuously changing alloy microstructure during solidification. Further, the permeability of the network plays a vital 
role in shrinkage accommodation and the possibility of forming defects. Hot tearing is a common defect that occurs during the solidification of alloys. A detailed discussion on such defects is presented in the following section.

\subsection{Hot Tearing}

Hot tearing is a common and serious defect occurring during the solidification of castings. The previous section demonstrated the role of developing alloy microstructure during solidification on the permeability of the solidifying network and subsequent formation of defects such as hot tears. Various feeding mechanisms during solidification help to accommodate the shrinkage of solidifying castings. However, some instances can occur whereby the buildup of stress within a casting leads to the formation of hot tears. Generally, the stresses responsible for hot tear formation are thermal stresses (which are generated during solidification) and mechanical stresses (which result from restrained contraction of a casting with a rigid mold).

The subject of hot tearing has been extensively studied and many theories have been proposed. Comprehensive reviews on hot tearing have been carried out by Eskin et al. [6], Sigworth [7] and Li et al. [8]. It has been shown that hot tearing is a complex phenomenon with many variables influencing their formation. Such variables include alloy composition, alloy-cooling rate, casting process parameters (i.e. melt superheat and mold temperature) and casting design. Further, a fine grain structure has been shown to limit the formation of hot tears. The following sections summarize the proposed theories of hot tearing, the factors affecting hot tearing and the available assessment methods. Although hot tears are found to occur in both ferrous and non-ferrous alloy systems, the focus of this review will be mainly on the non-ferrous alloy systems (i.e. aluminum and magnesium alloys).

\subsubsection{Theories of Hot Tearing}

Some of the first significant contributions to hot tearing research were presented by Verö [9] in the 1930's. He attempted to correlate the mechanical properties of solidifying alloys with hot tearing by carrying out both bend tests and tensile tests on Al-Si alloys at elevated temperatures. 
Unfortunately, as he was unable to generate reproducible results with the tensile test, he eventually discarded it in favour of the bend test. In turn, he found that the bending strength of various Al-Si alloys decreased rapidly with increasing temperature. However, he was unable to effectively distinguish between the drop in strength for a hot tear prone alloy to the drop in strength for a less prone alloy and eventually abandoned his attempts.

Singer and Cottrell [10] followed up on the pioneering research conducted by Verö by performing tensile tests on Al-Si alloys in the region of solidus. The authors confirmed the findings of Verö and identified "a range of temperature above the solidus over which some alloys have a finite strength or coherence, while at the same time having a negligible ductility" [10]. This temperature range was termed the "hot-short temperature range" and was considered by the authors to be one of the most important factors in determining the hot-shortness (or hot tearing) properties of alloys. At temperatures above this range, it was found that the alloys were not coherent as they collapsed over their own weight, while at temperatures below the hot-short temperature range, the alloy ductility was too high and the resulting fracture surfaces were not typical of hot tear surfaces.

The work of Singer and Cottrell spurred further investigation by Pumphrey and Jennings [11]. In this study, the authors suggested that at temperatures slightly above solidus, a film of liquid separates the coherent dendrite network and failure takes place at a stress lower than the strength of the individual crystals. It was observed that failure occurs in this region with very little plastic deformation. As a result, this region of temperature between that at which a coherent dendrite structure forms and the solidus temperature was called the "brittle temperature range" [11]. The authors believed that hot tearing occurred during this temperature range as a result of contraction stresses. During solidification the temperature gradients present along the casting would result in the failure of the casting's hottest (weakest) region, because of the stress imposed from the contraction of surrounding (cooler) regions.

Bishop et al. [12] studied the initiation of hot tears by simultaneously using radiography and thermal analysis of solidifying castings. The authors established that hot tearing occurs at temperatures near solidus at which solidification is complete except for the presence of liquid 
films. The liquid films surround the coherent dendrite network, as seen by Pumphrey and Jennings, and contribute to the decreased strength of the network. The basic mechanism for hot tearing was said to be the separation of dendrites at temperatures approaching solidus where only a minute amount of liquid remains. The authors referred to this stage of solidification as the film stage. It was at this time when Pellini $[13,14]$ developed his strain theory of hot tearing, which suggested that both strain accumulation and the presence of liquid films were factors responsible for hot tearing. Specifically, he believed that hot tearing was a strain-controlled phenomenon, which occurred if the accumulated strain in a hotspot reached a critical value. At the onset of the film stage, the liquid film is initially thick and continuous. At this stage, the load required to deform the hot spot (liquid film) should be near zero; but the deformation or extension required to open the liquid film and initiate a hot tear should be relatively high. As solidification progresses, the liquid film becomes thinner and the deformation is localized on a few remaining hot spots giving rise to a high strain. The accumulated strain at a hot spot was dependent on both the strain rate and the time required for a sample to pass through a film stage. Pellini [13] concluded that the liquid film provided the condition necessary for hot tearing and the actual occurrence of hot tearing was the result of mechanical factors contributing to the rate of deformation. Higher strain rates resulted in greater possibilities for hot tear formation.

Following the work of Pellini, Saveiko [15] developed a theory based on the liquid film between grains, whereby surface tension of the liquid film was deemed critical for hot tearing. He reasoned that during solidification, as shrinkage proceeds, both the solid grains within a solidifying network and the liquid film separating such grains would be subjected to extension. Once this extension reached a critical value of deformation, the liquid film would tear and a hot tear would form. Saveiko claimed that in order for this to occur, work must be done to overcome the molecular cohesion forces and such work was dependent on the surface tension of the liquid film [15]. Thus, he believed that the force $P$, required to tear the liquid film was given by:

$$
P=2 \alpha F / 1000 b
$$

Where $\alpha$ is the surface tension of the liquid, erg $/ \mathrm{cm}^{2} ; F$ is the area of contact between the plates and liquid, $\mathrm{cm}^{2} ; b$ is the thickness of the liquid film between the plates, $\mathrm{cm}$. 
Saveiko [15] also stressed the importance of the liquid film thickness and stated that it was a function of grain size and alloy purity. He felt that as the grain size and impurity decreased, the film became thinner. Since the tearing force required in Equation 1 is inversely proportional to the film thickness, the hot tear resistance increases. The effect of grain size on hot tearing is discussed in detail in Section 2.2.2.2.

The liquid film surrounding grains was also found to be a contributing factor of hot tearing in separate studies carried out by Novikov (as described by Eskin [6]), Pumphrey and Lyons [16] and Prokhorov [17]. Novikov believed that under low strain rates, grain boundary sliding was the main mechanism of hot tearing. They suggested that the liquid film surrounding the grains would act as a lubricant and enable grain boundary displacement, which in turn, could effectively accommodate the applied load on the semi-solid body. This was in direct contrast to Pumphrey and Lyons [16], who suggested that liquid along grain boundaries permitted the free movement of crystals to accommodate casting and thermal contraction, and therefore, prevent hot tearing. Meanwhile, Prokhorov [17] felt that an increase in film thickness increased the strain required to fracture or separate interdendritic regions and form hot tears.

Metz and Flemings [18] carried out a fundamental study on hot tearing in $\mathrm{Al}-\mathrm{Cu}$ and $\mathrm{Al}-\mathrm{Si}$ alloys. The authors explored the relationship between the response to imposed strain of the solidifying alloys and the formation of hot tears. In their study, liquid-solid mixtures were strained in shear using various strain rates. The strain rate was found to be a contributing factor to hot tearing. The authors concluded that at higher strain rates interaction between dendrites resulted in the development of a plastically deformed structure in the casting that resists further strain. The formation of hot tears was then the result of a progressive separation of dendrites to accommodate strain [18].

A group of researchers considered hindered feeding of the solid phase by the liquid as the main cause of hot tearing $[19,20]$. These researchers suggested that hot tearing would not occur as long as there is sufficient feeding of liquid metal during solidification. In this context, Clyne and Davies [21] proposed a theory involving a consideration of the time during solidification in which processes related to crack formation might take place. The authors believed that during the 
mass feeding and interdendritic feeding stages of solidification, the strain imposed on a casting could be accommodated by either solid movement or liquid movement, respectively. However, at the late stages of solidification, the dendrites begin to bridge and are no longer able to move freely. As a result, the applied strain leads to hot tearing. Based on this theory, a CSC (crack susceptibility criterion) was proposed, as shown in Equation 2.

$$
C S C=t_{v} / t_{r}
$$

Where $t_{v}$ was defined as the time interval in which the solidifying alloy is prone to hot tearing, in $\mathrm{s}$, while $t_{r}$ represented the time available for stress relief, in $\mathrm{s}$.

The parameter $t_{r}$ corresponded to the time spent in the volume fraction liquid region of 0.6 to 0.1 , where mass and liquid feeding readily occur. The parameter $t_{v}$, on the other hand, was the time interval whereby the alloy is more prone to cracking and lied between the volume fraction liquid range of 0.1 to 0.01 . The time period between a volume fraction liquid of 0.01 to 0 was assumed by Clyne and Davies [21] to be a region where no cracking would occur because the material is mostly solid at this point and is, therefore, too strong to crack. Hence, the vulnerable region $t_{v}$, was selected to lie between the aforementioned 'no crack region' and $t_{r}$. These regions are displayed graphically in Figure 2-2.

More recently, it was suggested by Sigworth [7] that liquid metal embrittlement might be responsible for hot tearing. With reference to Griffith's crack theory, Sigworth claimed that the strain energy stored in a material under tension is assumed to be released as energy needed to create new surface area where cracks grow. In ductile materials, most of the fracture energy is consumed in plastic deformation at the root of the growing crack tip. However, when certain liquid metals are present, the ductility nearly vanishes, the fracture stress decreases and the cleavage energies calculated from Griffith's crack theory are very close to the measured surface free energies. As a result, he concluded that liquid metal embrittlement (and also hot tearing) is caused by the surface-free energy between the liquid and solid (at grain boundaries) being small enough to easily create liquid cracks [7]. Sigworth noted that although liquid metal is not 
introduced to the solid in hot tearing, like it is in liquid metal embrittlement, during hot tearing a portion of the alloy is molten and thus, liquid is already present.

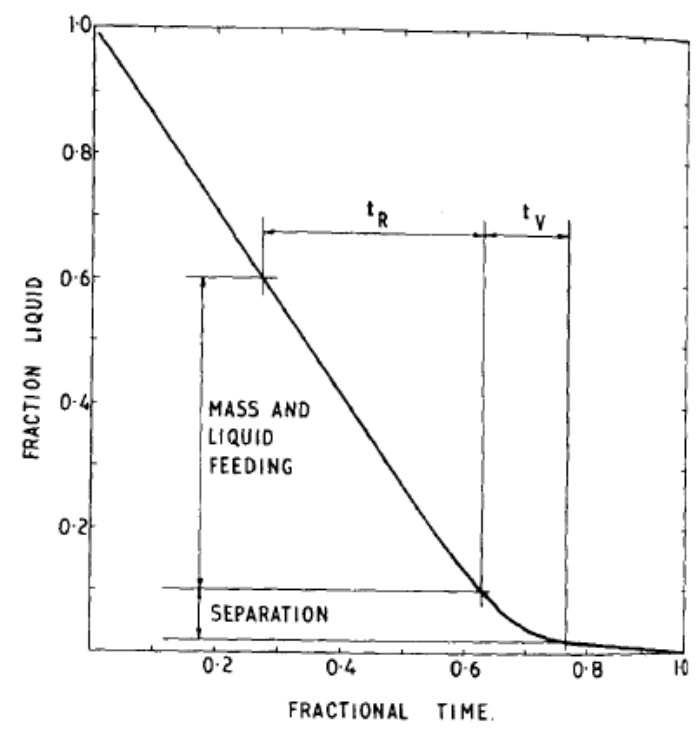

Figure 2-2. Graphical representation of the vulnerable time period, $t_{v}$ and the stress relief period, $t_{r}$ [21].

\section{Section Summary}

Hot tearing has been extensively studied over many decades and numerous theories on the formation of hot tears have been proposed. Such theories demonstrate the complexity of this phenomenon as it was proposed that stress, strain, strain-rate and liquid metal feeding all are possible underlying mechanisms in hot tear formation. The results of the current thesis are compared to some of these proposed theories. The formation of hot tears is also shown to be strongly dependent on both intrinsic factors (e.g. strength and ductility of solidifying alloy) and extrinsic factors (e.g. restriction of casting contraction by a rigid mold). However, despite such confusion, it follows from the discussion that in order for hot tears to occur, there must be a state of solidification where solid grains of the alloy casting are surrounded by a film of liquid metal. In turn, metallurgical factors affecting the morphology of grains and subsequent distribution and amount of liquid film collectively alter the hot tearing resistance of an alloy. The following sections address such factors. Specifically, the effect of alloy composition, grain size and morphology and casting process parameters are discussed. 


\subsubsection{Factors Affecting Hot Tearing}

This section describes the various factors affecting the formation of hot tears. Specifically, the effects of alloy composition, grain size and morphology and casting process parameters are addressed.

\subsubsection{Effect of Alloy Composition}

The first systematic study on the effect of alloy composition on hot tearing was carried out in the 1930's by Verö [9]. He systematically varied the amount of Si content in the Al-Si binary alloy system and investigated the resulting effects on hot tearing severity. The hot tearing severity was rated by measuring the observed crack length(s) on the produced castings. It was found that initial amounts of $\mathrm{Si}$ (up to $1.6 \mathrm{wt} \%$ ) resulted in an increase in the hot tearing severity (crack lengths), while further amounts (beyond $1.6 \mathrm{wt} \%$ ) decreased the hot tearing severity.

The research carried out by Verö was extended to other Al alloy systems by Pumphrey and Lyons [16]. Analysis was carried out on six Al binary alloys: Al-Si, Al-Cu, Al-Mg, Al-Fe, $\mathrm{Al}-\mathrm{Mn}$ and Al-Zn. Again, the total measured crack length on the final castings was used to evaluate the hot tearing severity. The results showed a similar pattern to that observed by Verö in Al-Si alloys. In all alloy systems, the hot tearing severity was found to initially increase before decreasing again after a certain content of solute. The amount of solute necessary to reduce hot tearing severity varied for each alloy system.

In a study carried out by Rosenberg et al. [19], a test was developed to examine the severity of hot tearing in three binary $\mathrm{Al}$ alloys (Al-Cu, $\mathrm{Al}-\mathrm{Mg}$ and $\mathrm{Al}-\mathrm{Sn}$ ) and two binary $\mathrm{Mg}$ alloys ( $\mathrm{Mg}-\mathrm{Al}$ and $\mathrm{Mg}-\mathrm{Zn})$. The authors developed a technique in which the hot tear resistance was rated by the maximum length of test casting which could be produced free of tears; the greater the length, the greater the resistance to hot tearing. The lengths of pure $\mathrm{Al}$ and pure $\mathrm{Mg}$ castings were measured at $\sim 12$ inches $(30.5 \mathrm{~cm})$. With small additions of solute to the pure metals the hot

tearing resistance decreased. Various alloying elements had different effects on hot tear resistance, as some were more severe than others. In all alloy systems investigated, the hot tear 
resistance was lowest for one or more solute compositions in the range of $0.25-10 \mathrm{wt} \%$. Specifically, minimum hot tear resistance was observed at compositions ranging from 0.25-5 wt\% Sn, $5 \mathrm{wt} \% \mathrm{Cu}$ and 4-6 wt\% $\mathrm{Mg}$ in the $\mathrm{Al}-\mathrm{Sn}, \mathrm{Al}-\mathrm{Cu}$ and $\mathrm{Al}-\mathrm{Mg}$ alloy systems, respectively. Beyond the lowest point of hot tear resistance, subsequent increases in solute contents were effective at increasing the hot tearing resistance.

Plots of cumulative crack lengths versus concentration levels were generated for $\mathrm{Al}-\mathrm{Cu}, \mathrm{Al}-\mathrm{Si}$, $\mathrm{Al}-\mathrm{Mg}$ and $\mathrm{Al}-\mathrm{Zn}$ alloys in a study carried out by Davies [22]. The resulting plots are shown in Figure 2-3. Maximum crack lengths were observed at $\sim 2 \mathrm{wt} \% \mathrm{Cu}, \sim 0.5 \mathrm{wt} \% \mathrm{Si}, \sim 0.75 \mathrm{wt} \% \mathrm{Mg}$ and $\sim 4 \mathrm{wt} \% \mathrm{Zn}$ for the respective alloy systems. Beyond that point, the hot tearing resistance was seen to increase again, with the exception of the Al-Zn alloy, whose resistance initially increased prior to decreasing again with levels of $\mathrm{Zn}$ beyond $6 \mathrm{wt} \%$. The results of this study were in close agreement to those of Pumphrey and Lyons [16], but varied from those of Rosenberg et al. [19]. Davies [22] did not address the reasons for which diverse solute contents of maximum hot tearing susceptibility were observed in his study in comparison to that of Rosenberg et al. Such differences however, may have been due to the diverse test method and casting process parameters used in each study. Further discussion on the effect of these factors is provided in later sections. Nevertheless, despite the differences observed in solute compositions, similar trends of increasing and decreasing hot tearing severity were again seen in this study. These trends are known as lambda curves, as a result of their shape, and are typical for most binary alloys.

Prior to the research of Davies [22], it was generally accepted that the alloy freezing range was the major factor responsible for the different hot tearing resistances observed in various binary alloy systems. Longer freezing range alloys were expected to have a higher susceptibility to hot tearing than those alloys with shorter freezing ranges. In particular, the alloy composition with the greatest temperature interval between solidus and liquidus, and thus, that which corresponded to maximum solid solubility of alloying element, was considered the most susceptible to hot tearing [16]. Davies, however, suggested that the severity of hot tearing of an alloy was dependent on its value of the equilibrium distribution coefficient (i.e. the ratio of the solid solubility to the liquid solubility at a given temperature in the phase diagram). He believed that 
the higher the value, the greater the "intensity" of segregation that occurs along grain boundaries, and the greater the likelihood of hot tearing [22].
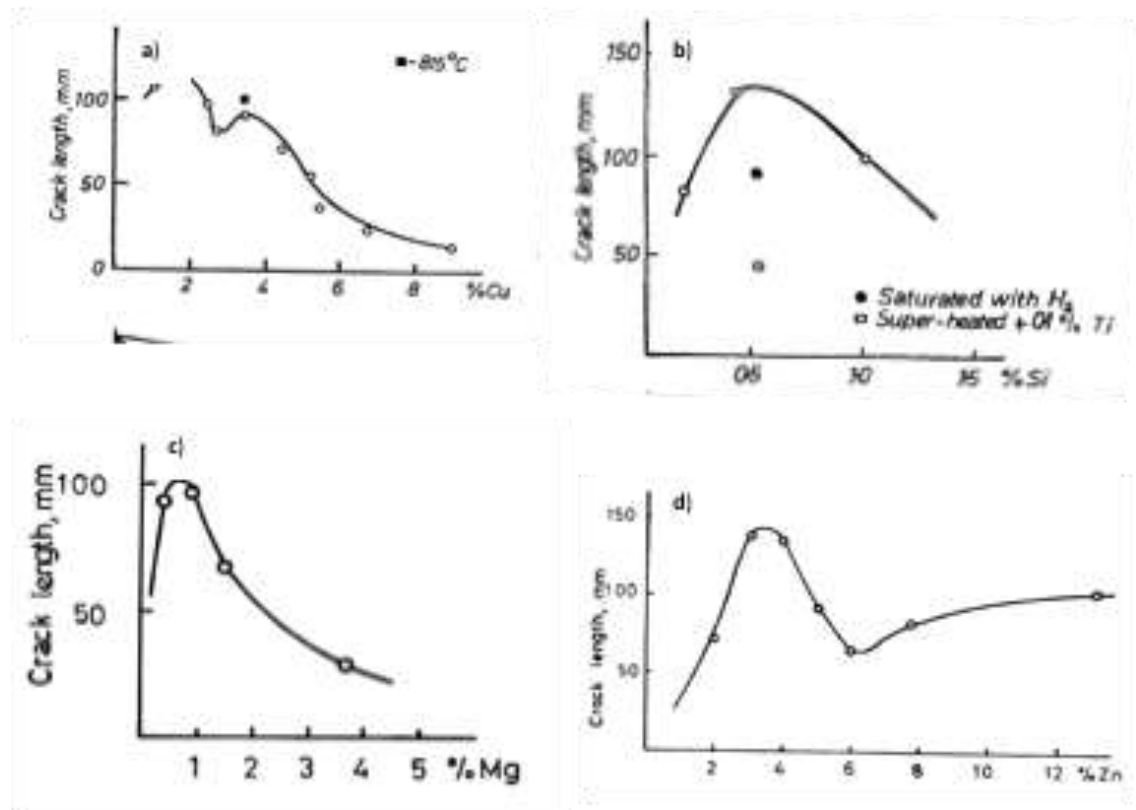

Figure 2-3. Effect of solute content on hot tearing in a) Al-Cu alloys, b) Al-Si alloys, c) Al-Mg alloys and d) Al-Zn alloys [22].

Clyne and Davies [23] carried out an in depth analysis on the effect of $\mathrm{Mg}$ content on hot tearing of Al. They developed a technique which used electric resistance to measure the cracks present in a casting. Such measurements were then plotted against Mg content. Three different pouring temperatures were used as well. The resulting lambda curves are shown in Figure 2-4. The hot tearing susceptibility of $\mathrm{Al}$ was found to be heavily influenced by both $\mathrm{Mg}$ content and superheat. Increases in superheat decreased the $\mathrm{Mg}$ content range attributed to maximum cracking, as shown in Figure 2-4. Maximum hot tearing was observed at $1 \mathrm{wt} \% \mathrm{Mg}$ at $50{ }^{\circ} \mathrm{C}$ superheat, then decreased to $0.75 \mathrm{wt} \% \mathrm{Mg}$ at $100{ }^{\circ} \mathrm{C}$ superheat and finally to $0.5 \mathrm{wt} \% \mathrm{Mg}$ at $150{ }^{\circ} \mathrm{C}$ superheat. Similar effects of superheat were observed in Al-Cu alloys in a study carried out by Spittle and Cushway [24].

The authors investigated the effect of $\mathrm{Cu}$ content on hot tearing of $\mathrm{Al}$ along with the role of varying superheat [24]. Hot tears were found to occur in all castings with $\mathrm{Cu}$ contents ranging from $0-7 \mathrm{wt} \%$. However, the superheat was seen to have a significant influence on the 
composition range for which hot tears were observed. With higher superheats, the composition of maximum cracking observed in the lambda curve, was found to decrease to a lower $\mathrm{Cu}$ content. As the content of $\mathrm{Cu}$ exceeded $7 \mathrm{wt} \%$, minimal to zero cracking was observed irrespective of superheat. The authors attributed this to an increase in volume fraction of eutectic (with increase in $\mathrm{Cu}$ concentration), which enhanced interdendritic feeding and effectively healed developing hot tears [24]. Oya et al. [25] concluded similarly in their study on Al-Cu alloys, but healing was observed only upon reaching $\mathrm{Cu}$ contents in excess of $20 \mathrm{wt} \%$.

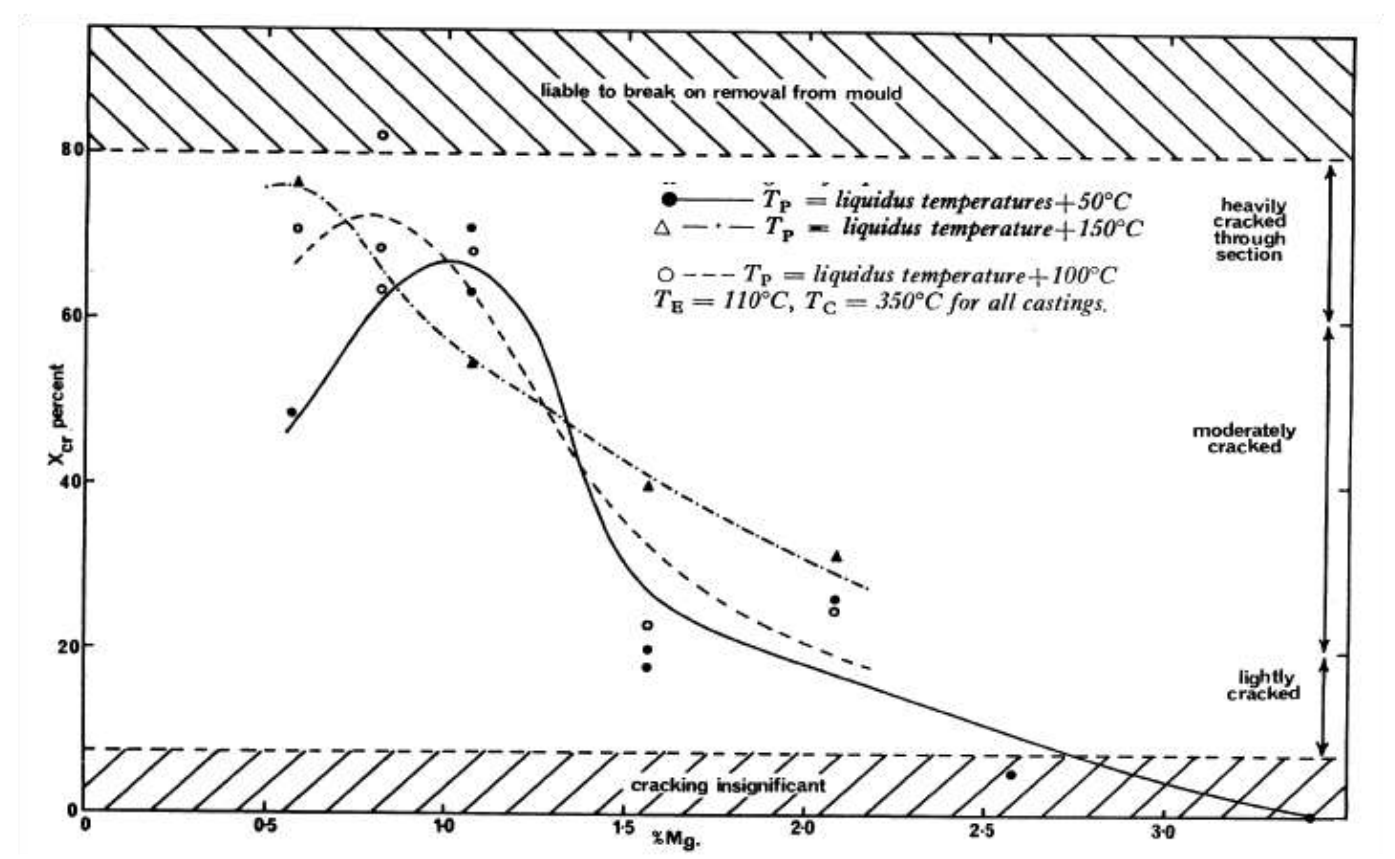

Figure 2-4. Lambda curves observed for Al-Mg alloys at varying superheats [23].

Other researchers $[16,19,26]$ have also demonstrated the importance of the eutectic phase and its effect on hot tearing. Rosenberg et al. [19] suggested that at low solute contents, 'pockets' or 'films' of liquid eutectic reduced the strength of near solid castings and subsequently lowered hot tearing resistance. As the solute content increased, however, the authors claimed that enough eutectic formed to completely surround primary grains and fill incipient hot tears. Pumphrey and Lyons [16] were in agreement with Rosenberg et al. and insisted that with increasing amount of eutectic, stress accommodation and healing of hot tears were critical. Eskin et al. [26] also believed that alloy composition plays an essential role in the occurrence of hot tears because of the amount of liquid available for feeding at the late stages of solidification. Specifically, they 
stated that increased amount of eutectic plays a decisive role, as eutectic liquid is the last liquid available for feeding and healing hot tears.

The interaction between alloying elements has been also found to be a critical factor in hot tearing, as demonstrated by the work of Chamberlain et al. [27] and Sigworth et al. [28]. In the two studies, the hot tearing characteristics of Al-Mg-Zn alloys were found to be significantly dependent on the $\mathrm{Mg}: \mathrm{Zn}$ ratio. Both studies showed that the propensity to tearing of $\mathrm{Al}-\mathrm{Mg}-\mathrm{Zn}$ alloys significantly decreased with increasing $\mathrm{Mg}: \mathrm{Zn}$ ratio. Specifically, no hot tears were seen in castings with $\mathrm{Mg}: \mathrm{Zn}$ ratios of 1.4:1 or greater.

The effect of alloy composition on hot tearing has also been examined in Mg alloys. Pekguleryz and Vermette [29] carried out a systematic study on the hot tearing susceptibility of various $\mathrm{Mg}$ die casting alloys. Their results showed that Mg-Al alloy AZ91D was most resistant to hot tearing while Mg-RE alloy AE42 was most susceptible. The authors attributed the differences in hot tearing resistance to the differences in freezing ranges and eutectic fraction between the alloys. Alloys with both freezing ranges near $100{ }^{\circ} \mathrm{C}$ and high eutectic fractions were found to be more resistant to hot tearing than those containing both freezing intervals well above or below $100{ }^{\circ} \mathrm{C}$ and low eutectic fractions [29].

More recent investigations on the Mg-RE alloy system have also been carried out. The effect of Gd and $\mathrm{Y}$ on hot tearing of Mg alloys was examined in separate studies [30-32]. In all studies, it was found that low contents of $\mathrm{Gd}(<2 \mathrm{wt} \%)$ and $\mathrm{Y}(<0.9 \mathrm{wt} \%)$ resulted in increased susceptibility to hot tearing. This was mainly attributed to two factors: an increase in grain size and subsequent columnar grain structure, and reduced amount of eutectic liquid available at the time of cracking. In contrast, higher amounts of $\mathrm{Gd}$ (up to $10 \mathrm{wt} \%$ ) and $\mathrm{Y}(>1.5 \mathrm{wt} \%$ ) were seen to improve the hot tearing resistance of $\mathrm{Mg}$. With increased $\mathrm{Gd}$ and $\mathrm{Y}$, the grains transformed into a more equiaxed structure and there was a greater amount of eutectic $\mathrm{Mg}-\mathrm{Gd}$ and $\mathrm{Mg}$-Y liquid readily available to heal developing hot tears.

\section{$\underline{\text { Section Summary }}$}

Numerous investigations carried out on the effect of alloy composition on hot tearing were discussed. It was found that in most binary alloys, the trend of cracking severity versus solute 
concentration shows an initial increase in cracking with increasing solute, followed by a decreasing trend. Such a trend is known as a lambda curve, and was generally attributed to alloy freezing ranges, segregation and amount of eutectic. Further, in some cases the addition of solute was seen to alter the grain structure of the alloy from columnar to equiaxed. Further details on the effect of grain structure on hot tearing are provided in the next section.

\subsubsection{Effect of Grain Size and Morphology}

Davies [22] was one of the first researchers to carry out a systematic study on the effect of grain refinement on hot tearing. In this research, the grain size of a series of binary Al alloy castings was varied by modifying the amount of solute in each alloy, and by additions of $\mathrm{Ti}$ and Be. Further, a specialized ring mold was used to examine the hot tearing severity of each casting. More details on this apparatus are provided in Section 2.2.3.1. The resulting hot tears present on each casting were then measured and plotted against the grain size of each casting, as shown in Figure 2-5. It was found that for all alloy systems, the hot tearing severity (crack length) significantly increased with increased grain size. Davies [22] concluded that grain size was the single most contributing factor responsible for hot tearing.

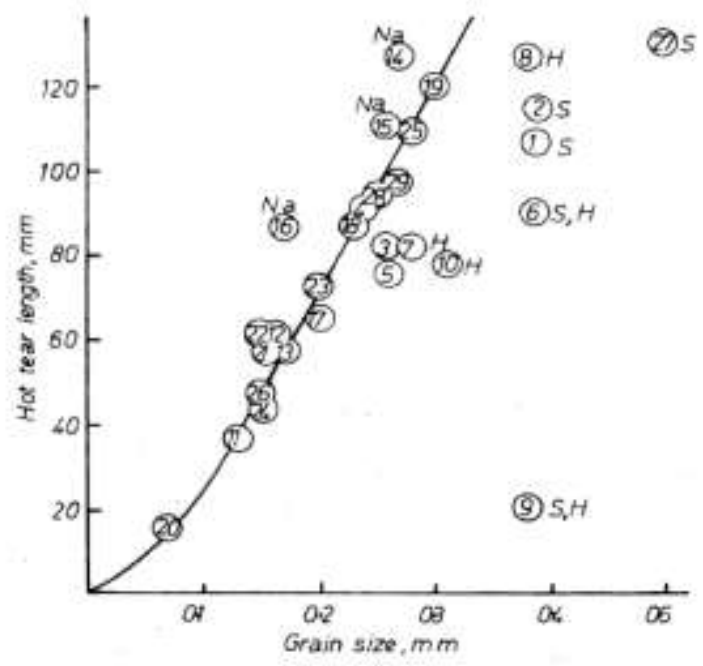

Figure 2-5. Plot showing increasing length of hot tears with increasing grain size for various Al alloys [22]. In the same year, Metz and Flemings [18] investigated the effect of grain refinement on hot tearing in $\mathrm{Al}-\mathrm{Cu}$ alloys. They found that grain refinement improved resistance to hot tearing by improving the alloy's ability to accommodate local strains. The authors suggested that small 
globular grains resulting from $\mathrm{Ti}$ additions were able to slide relative to one another, and alleviate the solidification strain. A similar study carried out by Braccini et al. [33] confirmed the findings of Metz and Flemings. In this study, the authors concluded that two mechanisms were responsible for the beneficial effect of grain refinement on minimizing hot tearing. The first mechanism was similar to that found by Metz and Flemings, where small globular grains were found to enable accommodation of thermal strain. The second mechanism was related to the permeability of the network through interdendritic regions. The authors suggested that in unrefined alloys, relatively coarse secondary dendrite arms entrap liquid between solid grains. Thus, the amount of available liquid to enable long-range intergranular feeding is low. In a grain refined structure however, the secondary dendrite arms and solid grains are less branched and the interdendritic network permeability is enhanced, along with interdendritic feeding.

In the investigation carried out by Pumphrey and Lyons [16] on binary Al alloys, grain refinement was obtained through increased solute additions. The authors found that increased levels of solute decreased grain size and subsequently increased hot tearing resistance. However, they felt that decreased grain size helped to improve an alloy's resistance to hot tearing by modifying the distribution of eutectic. They suggested that the finer the size of the primary crystals, the greater the probability of the eutectic being present at their boundaries. This was seen as advantageous because the eutectic at the boundary would permit free movement of the primary crystals and prevent hot tears from forming (recall Section 2.2.1).

Warrington and McCartney [34] investigated the influence of grain size and structure on hot tearing in an Al-6 wt\% Cu binary alloy. Titanium was added in levels ranging from 0-0.07 wt\%. It was found that small amounts of $\mathrm{Ti}(<0.05 \mathrm{wt} \%)$ reduced hot tearing severity significantly with respect to the unrefined alloy. However, at levels beyond $0.05 \mathrm{wt} \%$, some evidence of increased hot tearing tendency was observed. This was attributed to the alloy grain structure. The unrefined alloy possessed a columnar grain structure that was found to be significantly more susceptible to cracking than the equiaxed structures observed in the Ti-containing alloys. However, differences in grain structure were observed in the Ti-containing alloys as well. For instance, the alloys with small amounts of $\mathrm{Ti}(<0.05 \mathrm{wt} \%)$ contained equiaxed-dendrite 
structures, which were less susceptible to cracking than the equiaxed-cellular morphology found in the alloys containing levels of Ti greater than $0.05 \mathrm{wt} \%$.

Warrington and McCartney [35] extended their studies to Al alloys 7050 and 7010 (Al-Zn-Mg-Cu alloys) to confirm the influence of grain structure observed in the aforementioned investigation. In this case, additions of $\mathrm{Ti}$ ranging from $0-0.05 \mathrm{wt} \%$ were made and similar results were obtained. Again, it was found that the columnar grain structure of the unrefined alloy triggered a high susceptibility to hot tearing in both alloys. With low additions of Ti (e.g. 0.015-0.03 wt\%) the transformation of grain structure from columnar to equiaxed-dendritic significantly reduced the hot tearing severity in each alloy. However, additions of Ti beyond $0.03 \mathrm{wt} \%$ promoted the formation of an equiaxed-cellular grain structure in each alloy, and the hot tearing severity increased again. The authors postulated that such a discrepancy in hot tearing susceptibility was due to the poorer interlocking, towards the end of solidification, between essentially unbranched cellular grain surfaces as compared with the highly branched equiaxeddendritic network [35]. They believed that this would enable cracks to propagate more readily under the imposed stresses.

A study carried out by Easton et al. [36] displayed a similar relation between grain structure and hot tearing susceptibility in $6061 \mathrm{Al}$ alloy. The authors found that additions of Ti effectively decreased the grain size and transformed the grain structure from large columnar dendrites to a cellular dendritic structure without extensive dendrite branching. Additional grain refinement resulted in cellular grain morphology without dendrites. Hot tearing susceptibility was decreased as the grain morphology became cellular. However, similar to that which occurred during Warrington and McCartney's investigations, when further refinement of the grains was obtained, the hot tearing tendency increased again. In this case, the increased hot tearing tendency was attributed to a decrease in the permeability of the liquid through the microstructure with excessive grain refinement. However, the authors did not explain the mechanisms in detail.

Clyne and Davies [23] examined the effect of grain refinement on hot tearing in "low susceptibility" and "high susceptibility" Al-Mg alloys. They found that in an Al-1wt\% Mg alloy, or "low-susceptibility alloy", the hot tearing tendency increased over a narrow range of Ti 
additions, despite the transformation of grain structure from columnar to equiaxed. In the case of an Al-2wt\% Mg alloy, or "high-susceptibility alloy", the hot tearing tendency was found to be unaffected by $\mathrm{Ti}$ additions except at high levels (beyond $0.02 \mathrm{wt} \%$ ) even though the grain structure was altered from columnar to equiaxed. The authors concluded that there was a complex interaction between impurity content, grain structure and cracking susceptibility [23].

Spittle and Cushway [24] studied the effect of grain refinement on hot tearing in Al-Cu alloys with varying levels of $\mathrm{Cu}$ additions and superheat. The influence of grain refinement was found to be dependent on both superheat and alloy composition. Their results showed that grain refinement at a superheat of $250{ }^{\circ} \mathrm{C}$ reduced the susceptibility to hot tearing within the range of 1-7 wt\% $\mathrm{Cu}$. However, grain refinement was found to have little effect on susceptibility to tearing at $50{ }^{\circ} \mathrm{C}$ superheat for this composition range. Alloys containing more than $7 \mathrm{wt} \% \mathrm{Cu}$ were not found to be prone to hot tearing regardless of superheat and grain structure.

Other investigators $[19,37,38]$ studied the effect of grain refinement in Al-Cu alloys. Viano et al. [37] examined various levels of $\mathrm{Cu}$ at a constant superheat of $100{ }^{\circ} \mathrm{C}$. It was found that grain refinement slightly reduced hot tearing severity in the most susceptible solute content range of $0.25 \mathrm{wt} \% \mathrm{Cu}$ to $1 \mathrm{wt} \% \mathrm{Cu}$. However, for compositions greater than $1 \mathrm{wt} \% \mathrm{Cu}$, grain refinement did not have any significant effect on the hot tearing susceptibility. In a separate study carried out by Li et al. [38], grain refinement was found to significantly reduce hot tears in Al-Cu alloy B206. The authors attributed the improved resistance to hot tearing to increased feeding of liquid metal, a better capability of the grain refined structure to accommodate the generated stresses in the mushy zone and a decrease in the liquid film thickness between grains. Such findings were in contrast to those of Rosenberg et al. [19] who found that grain refinement in Al-Cu alloys showed no increased resistance to hot tearing.

Eskin et al. $[26,39,40]$ developed a technique to measure the thermal contraction of various Al alloys during solidification. The details of the experimental apparatus are discussed in Section 2.2.3.4.2. These authors considered thermal contraction as an important factor in the development of hot tears in a casting, and therefore attempted to correlate this factor to the alloy grain structure. In their studies, grain refinement was found to decrease both the amount of 
thermal contraction during solidification and the onset temperature of thermal contraction. As a result, the thermal contraction started at a later stage of solidification and less thermal strain was imposed on the mushy zone. This, in turn, reduced the overall hot tearing tendency of the investigated alloys.

Grain refinement has also been found to delay the onset of dendrite coherency [41-44]. In doing so, the mass feeding stage of solidification is enhanced and the solidifying network is therefore better fed with liquid. Further, a delay in the onset of dendrite coherency results in a decrease in the time interval in which the solidifying network contracts between the onset of dendrite coherency and final solidification. Such factors contribute to an overall reduced tendency to hot tearing.

\section{Section Summary}

A series of investigations on the effect of grain size and morphology on hot tearing were described. The results of past literature suggest that there is conflicting evidence on the subject. Some researchers suggested that grain refinement does not significantly impact hot tearing resistance of an alloy, while others felt that grain size and morphology are crucial to increasing an alloy's resistance. In the cases where grain refinement was found to have either no effect or a negative effect, the underlying mechanisms remain unclear. In contrast, those studies that demonstrate the ability of grain refinement to reduce hot tears suggest three underlying mechanisms: An improved ability of the alloy to accommodate the imposed stress and strain during casting; an enhanced permeability of the solidifying network; and finally, a delay in the onset of dendrite coherency. The current work aims to validate such mechanisms for the B206 alloy. The effects of melt superheat (pouring temperature) and mold temperature on hot tearing are discussed next.

\subsubsection{Effect of Casting Process Parameters}

It has been shown that hot tearing is strongly influenced by many variables including alloy content, amount of eutectic and grain size and structure. This section describes the effect of 
casting process parameters on hot tearing. Specifically, the effect of melt superheat (pouring temperature) and mold temperature are addressed.

\subsubsection{Effect of Melt Superheat}

Previous experimental research investigating the effect of melt superheat on hot tearing is sparse and conflicting. Pellini [13] addressed this issue when he stated: "at any meeting where the subject of hot tearing is discussed, the steel foundry community would divide into groups with distinctly opposite opinions regarding carbon and pouring temperature effects depending on the general types of castings produced and the foundry practices used by the individuals expressing the opinions". He referred to previous studies carried out on steel alloys, in which pouring temperature (melt superheat) was found to either increase or decrease hot tearing severity. The underlying reasons for such conflicting results were not known. These discrepancies, however, have also been observed in non-ferrous alloys.

The influence of melt superheat on the composition ranges for which Al-Mg alloys [23] and $\mathrm{Al}-\mathrm{Cu}$ alloys [24] were found to be susceptible to hot tearing was demonstrated in a previous section (recall Section 2.2.2.1). However, melt superheat was also found to have a significant impact on the hot tearing of alloys at fixed compositions. For example, in the case of Al-Cu alloys, Spittle and Cushway [24] found that higher melt superheat increased the film thickness along grain boundaries regions and therefore, in accordance with Pellini's strain theory [13], made the alloys more prone to hot tearing. Their results were in agreement to those of Pumphrey and Lyons [16] who observed that increases in melt superheat increased the severity of hot tearing in six binary Al alloys. On the other hand, Clyne and Davies [23] found that at fixed compositions, the hot tearing resistance of $\mathrm{Al}-\mathrm{Mg}$ alloys was unaffected by varying melt superheats. Further, a recent study by Bichler et al. [45] in AZ91D Mg alloy found that melt superheat did not have any significant effect on hot tearing.

The review of Li et al. [8] reported on the research of Couture and Edwards, who thought that two factors have a play in the controversy related to superheat and hot tearing. They suggested that increased superheat could either spread the hot spot and subsequently decrease hot tearing tendency; or increase the time for which liquid film is present and therefore increase the 
tendency to generate hot tears. Meanwhile, Spittle and Cushway [24] suggested that the conflicting findings could be the result of different methods designed to feed the castings (i.e. location of risers). In particular, the authors compared their results for the Al-7 wt\% $\mathrm{Cu}$ alloy to those of a previous study on the same alloy. They felt that the lack of influence observed with increased superheat in the previous study was due to the inability of liquid metal to feed the hot spot and compensate for solidification and contraction shrinkage.

\subsubsection{Effect of Mold Temperature}

The mold temperature is an important casting process parameter in that it directly controls casting cooling rate, which in turn, affects the resulting microstructure. As such, many researchers have preferred to keep mold temperature constant so as not to alter cooling rate or solidification time. However, in some instances, the mold temperature was systematically varied to observe the effects on hot tearing. A summary of some of the important studies is presented.

Bichler et al. [45] conducted an in depth analysis on the effect of mold temperature in AZ91D $\mathrm{Mg}$ alloy. The experiments were carried out using a pouring temperature of $700{ }^{\circ} \mathrm{C}$ and mold temperatures of $140{ }^{\circ} \mathrm{C}, 180{ }^{\circ} \mathrm{C}, 220{ }^{\circ} \mathrm{C}, 260{ }^{\circ} \mathrm{C}, 300{ }^{\circ} \mathrm{C}, 340{ }^{\circ} \mathrm{C}$ and $380{ }^{\circ} \mathrm{C}$. The mold temperature was found to have a significant impact on hot tearing severity. Specifically, increases in mold temperature effectively reduced hot tearing. The authors found $220{ }^{\circ} \mathrm{C}$ to be a "critical" mold temperature. At mold temperatures below $220^{\circ} \mathrm{C}$, the cracks were seen to initiate from all surfaces and connect across the entire cross section of the castings. On the other hand, at temperatures above $220{ }^{\circ} \mathrm{C}$, the cracks were hairline-like and did not connect. Finally, mold temperatures above $340{ }^{\circ} \mathrm{C}$ were sufficient to completely eliminate hot tears. The authors reasoned that increases in mold temperature improved bulk feeding of the casting and increased the size of interdendritic regions, which in turn enhanced flow of eutectic liquid at final stages of solidification. Further, it was suggested that casting contraction was more uniform at elevated mold temperatures.

Zhen et al. [46] investigated the effect of cooling rate on a binary $\mathrm{Mg}-1 \mathrm{Al}$ alloy using two mold temperatures $\left(250{ }^{\circ} \mathrm{C}\right.$ and $\left.500{ }^{\circ} \mathrm{C}\right)$. Elevated mold temperatures were effective at reducing hot 
tears, as no cracks were visible in castings produced with a $500{ }^{\circ} \mathrm{C}$ mold temperature. The authors suggested that higher cooling rates (attributed to lower mold temperatures) could create larger temperature gradients within a casting, thereby resulting in more severe hot spots and higher thermal stresses. Such factors would in turn increase hot tearing susceptibility. The authors extended such research to other $\mathrm{Mg}$ alloy systems and found that in both $\mathrm{Mg}-\mathrm{Gd}$ alloys [30] and Mg-Y alloys [32] higher mold temperatures successfully eliminated hot tears.

Fasoyinou et al. studied the effect of mold temperature on hot tearing in Al alloys 206 and 535. Li et al. [8] reported on their findings. The authors found that hot tearing was strongly dependent on mold temperature. Higher mold temperatures reduced hot tearing by providing an effective thermal gradient which in turn improved feeding of liquid metal. Further, simulation software was used in the study to calculate the strain developed in the mushy zone during the last stage of solidification. It was found that the principal strain was lower at higher mold temperatures and hence, a reduced tendency to form hot tears resulted. A study carried out by Sadayappan et al. [47] further illustrated the impact of mold temperature on hot tearing, as a minimum mold temperature of $350{ }^{\circ} \mathrm{C}$ was required to reduce the occurrence of hot tears in both $\mathrm{Mg}$ and $\mathrm{Al}$ alloys.

\section{Section Summary}

The effects of both melt superheat and mold temperature on hot tearing were presented. Past studies demonstrate conflicting results with respect to melt superheat, as higher melt superheats were seen to either increase hot tearing severity or have no effect. In contrast, the literature on the effect of mold temperature, though limited, generally portrayed a reduction of hot tearing with higher mold temperatures. Such studies further illustrate the complexity of the hot tearing phenomenon, since numerous factors are found to contribute to their formation. Another factor that may contribute to the complexity of hot tearing is casting design. Past researchers have used a variety of molds designed to assess the hot tearing resistance of alloys. The various designs could be an underlying reason for much of the contradictory results observed in similar alloys. The common assessment methods are discussed next. 


\subsubsection{Hot Tearing Test Methods}

Numerous methods were introduced to characterize the hot tearing behaviour of alloys. Such methods can be categorized into either qualitative or quantitative methods. This section describes the most common techniques used.

\subsubsection{Ring Mold Test}

A ring mold was commonly used to compare the hot tearing tendency of alloys. The mold, as shown in Figure 2-6, consists of a ring and a core made of materials with low thermal expansion coefficients and high melting points [6]. The ring and core are placed on top of a flat plate and liquid metal is poured in the space between the core and the ring. The resulting casting is shown in Figure 2-6 as well. In this setup, the core restricts the solidification shrinkage and thermal contraction of the solidifying alloy, which in turn, imposes tensile stresses and causes hot tears to form. Specifically, the restricting core generates a hoop stress perpendicular to the macroscopic growth front, which thereby causes tears to form parallel to the direction of solidification growth.

The ring mold setup was used in various studies [16,22] mainly because of its simplicity. In such studies, the combined length of all cracks observed on the produced castings was used as a means to characterize the hot tearing susceptibility of an alloy. One limitation of this assessment, as described by Davies [22], was that a large scatter in results was always observed, since a high number of small cracks gave rise to a higher susceptibility to hot tearing than one single crack through the entire cast ring. In contrast, Novikov and Grusko [48] varied the core diameters to alter the imposed stress on the solidifying alloys and used the length of the main crack to characterize the hot tearing susceptibility of the investigated alloys.

The limitations reported with the ring mold casting technique were a difficulty in controlling the solidification rate [48] and the requirement of the metal to be poured at the same level for each casting, to limit the effect of metallostatic head [22]. Besides that, the test gave only a qualitative value for hot tearing tendency. 


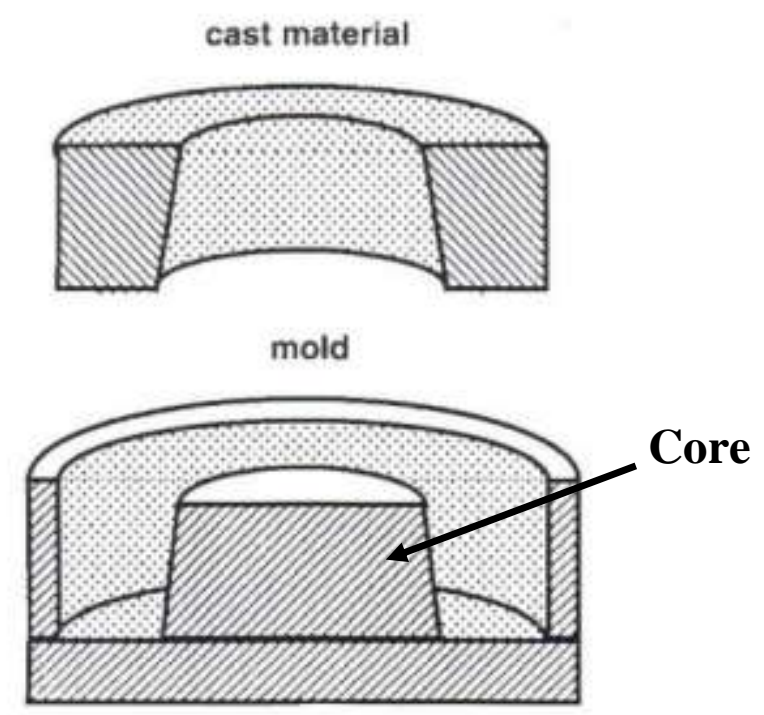

Figure 2-6. Ring mold test method and resulting ring casting [6].

\subsubsection{2 “Dog-Bone” Mold Test}

The "dog-bone" mold was given its name because of its close resemblance to the shape of a dog's bone. An example is shown in Figure 2-7 [6]. The mold is comprised of a casting bar with a restraint at each end. In this setup, the metal is poured in the centre of the mold and spreads towards the edges. Solidification begins from the edges and stresses develop in the centre region of the casting bar. In contrast to the ring mold, the "dog-bone" mold generates a stress perpendicular to the solidification growth. The susceptibility of an alloy to hot tearing was commonly characterized by measuring the observed crack length [6].

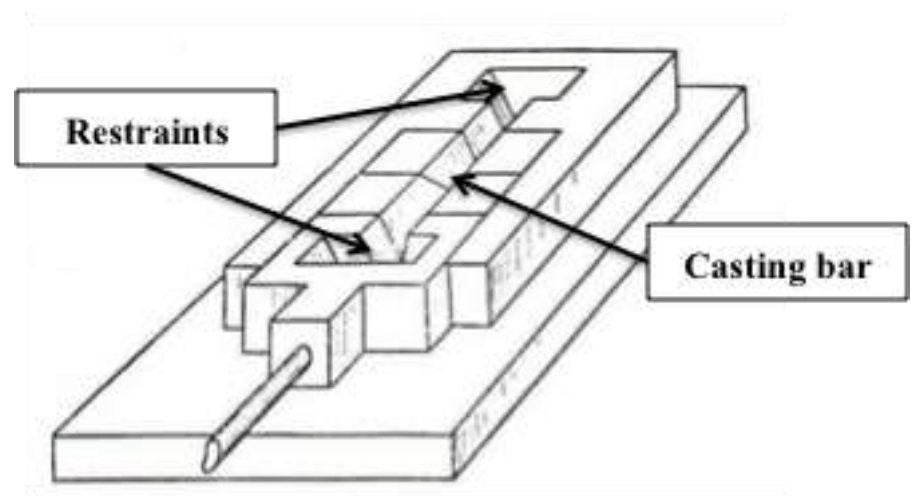

Figure 2-7. "Dog-bone" mold used to study hot tearing [6]. 
Eskin et al. [6] described modifications made to the mold in Figure 2-7, such as that shown in Figure 2-8. Here, several dog bone molds were combined in a single setup, with varying lengths. Hot tearing was found to occur in the longer samples, as a result of an increase in constrained shrinkage. Therefore, the hot tearing susceptibility was measured as the minimum critical sample length required for hot tear formation [6]. Similar modifications were used in various other studies as well $[27-29,47]$. Again, the minimum length required to form hot tears was used to estimate the hot tearing tendency.

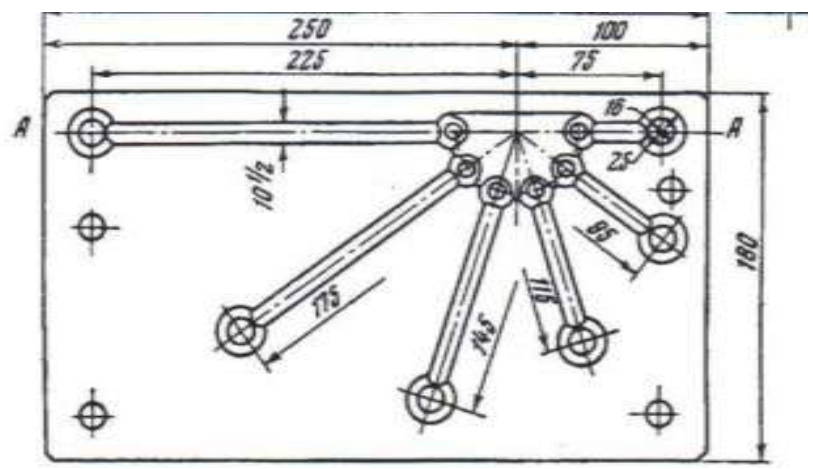

Figure 2-8. Modified "dog-bone" mold with samples of varying lengths (dimensions in mm) [6].

Another configuration of the "dog-bone" mold is shown in Figure 2-9 [6]. In this case, the diameter of the casting bars was varied from 4 to $16 \mathrm{~mm}$ for a constant length of $40 \mathrm{~mm}$. The hot tearing severity was measured as the maximum critical diameter of the sample in which hot tears evolved [6].

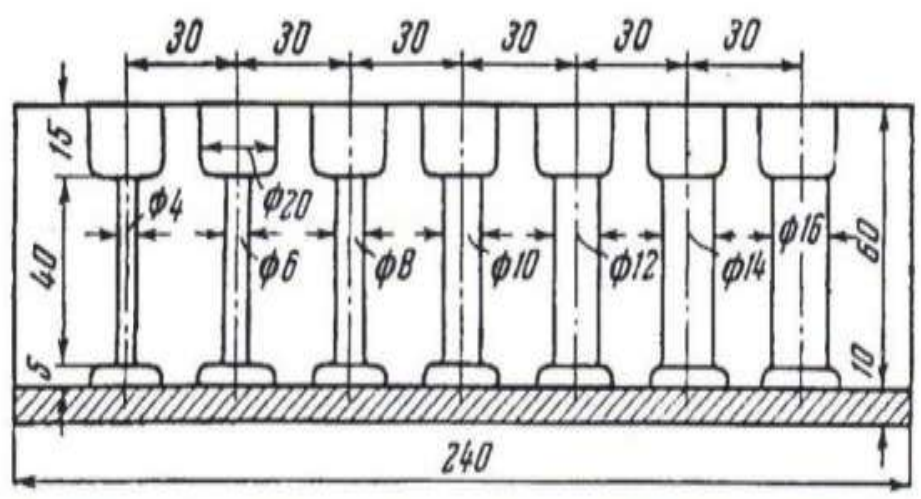

Figure 2-9. Modified "dog-bone" mold with varying sample diameters (dimensions in mm) [6]. 
Clyne and Davies [23] also reported a modified version of the "dog-bone" mold. In their study, a heater was placed in the centre of the mold and a cooler was inserted at each end by the restraints. This setup enabled a precise control of casting temperature and subsequent cooling rate. The authors also had the capability to reheat the melt and control thermal gradients within the casting. Spittle and Cushway [24] used a similar setup with water-cooled copper chills at the ends and a feeder placed in the middle near the hot spot of the casting. This mold was designed to simulate a real casting where a feeder is available to control the amount of liquid metal feeding and examined its effect on hot tearing. The authors felt that lack of feeding in some hot tearing tests influenced the trend observed when monitoring hot tearing tendencies as a function of a given variable [24]. In both studies [23,24], the hot tearing susceptibility was expressed by the value of the cross-sectional area of cracks determined from an electrical resistance measurement technique.

The advantages of the "dog-bone" mold setup stem from the simplicity of the design as well as the many possibilities for modification (i.e. varying lengths and diameters, placement of chills and heaters, etc.). However, despite such advantages, the results of hot tearing tendencies remain qualitative.

\subsubsection{Cold Finger Test}

The cold finger test was developed by Warrington and McCartney [34]. This technique was designed to mimic the macroscopic growth direction and direction of applied stress that occurs during direct chill (DC) casting of Al alloys. The authors felt that previous research on hot tearing susceptibility measurement ignored the relationship between such factors. Specifically, they suggested that both the hoop stress which developed perpendicular to the macroscopic growth front in the ring mold technique, and the stress that developed parallel to the macroscopic growth front in the 'dog bone' mold setup, were not suitable to simulate hot tearing during DC casting. As a result, the cold finger test, shown in Figure 2-10, was developed.

The test system consisted of an internally tapered steel crucible in an open-ended tube furnace and a separate water-cooled copper chill with a tapered conical portion. The molten alloy was 
held at desired temperature inside the crucible and the chill was then inserted into the melt to a pre-determined depth. As a result, the chill would act to restrict the solidification of the ingot, as the alloy solidified in a direction perpendicular to the chill's surface. Upon completion of solidification, both the chill and the adhering ingot were removed from the furnace and the ingot was then separated from the chill. The authors concluded that as a result of the tapered nature of the chill (or restraint), the distance the hot tear propagates down the side of the ingot was a direct measure of the alloy's tendency to form hot tears. Such an apparatus was used to investigate the hot tearing susceptibility of various alloys [34,35].

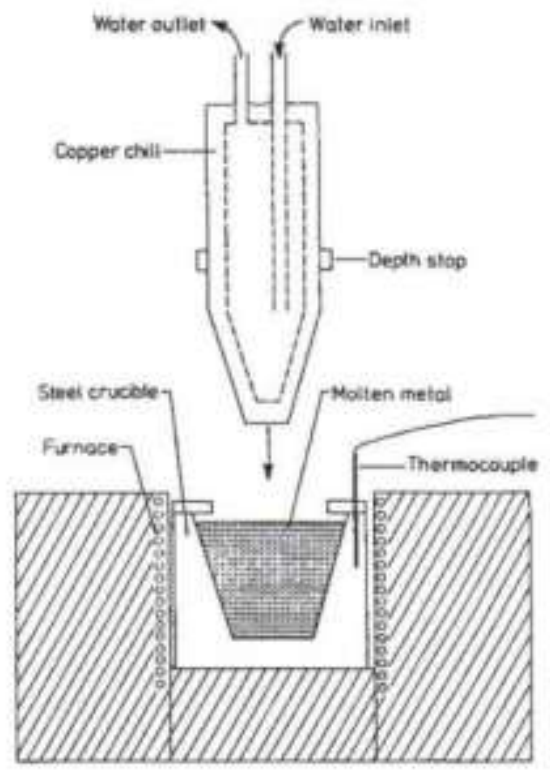

Figure 2-10. Cold finger test experimental setup [34].

\subsubsection{In Situ Methods}

The aforementioned techniques used for characterizing hot tearing susceptibility are relatively simple in design and therefore were commonly used in past research. However, the results generated from such techniques are only qualitative in nature. As such, new methods were developed to provide a more quantitative approach. In particular, two common techniques use a load cell and linear variable differential transformer (LVDT) to measure load (stress) and displacement during solidification, respectively. These measurements are then directly correlated to hot tearing susceptibility. The following section describes such methods. 


\subsection{Load Cell Method}

The load cell method was developed to quantitatively measure the contraction force of solidifying alloys and to relate such measurements to hot tearing. Instone et al. [49] incorporated a load cell in a constrained solidification test rig designed to characterize tensile strength development and hot tearing behaviour during solidification. The setup is shown in Figure 2-11. The mold was designed to simulate DC casting and consisted of a pouring reservoir at the centre, which ensured that hot tears form at the central location of the bars. One test bar was fully restrained and used for microstructural analysis and temperature measurements. The other test bar was used for load data collection and was only restrained at one end while the other end was connected to a load cell. A schematic of the hot tear rig is shown in Figure 2-12.

Viano et al. [37] also used this setup in his study on $\mathrm{Al}-\mathrm{Cu}$ alloys. The authors recorded temperature and load as a function of time in order to characterize the hot tearing susceptibility of the alloys. The load was measured at the solidus temperature (i.e. $548{ }^{\circ} \mathrm{C}$ ) for a series of $\mathrm{Al}-\mathrm{Cu}$ alloys [37]. The resulting plot is shown in Figure 2-13. The results showed good agreement to the hot tearing severity of the alloys, as a lambda relationship, similar to that observed in Section 2.2.1 for various alloys, was obtained. 


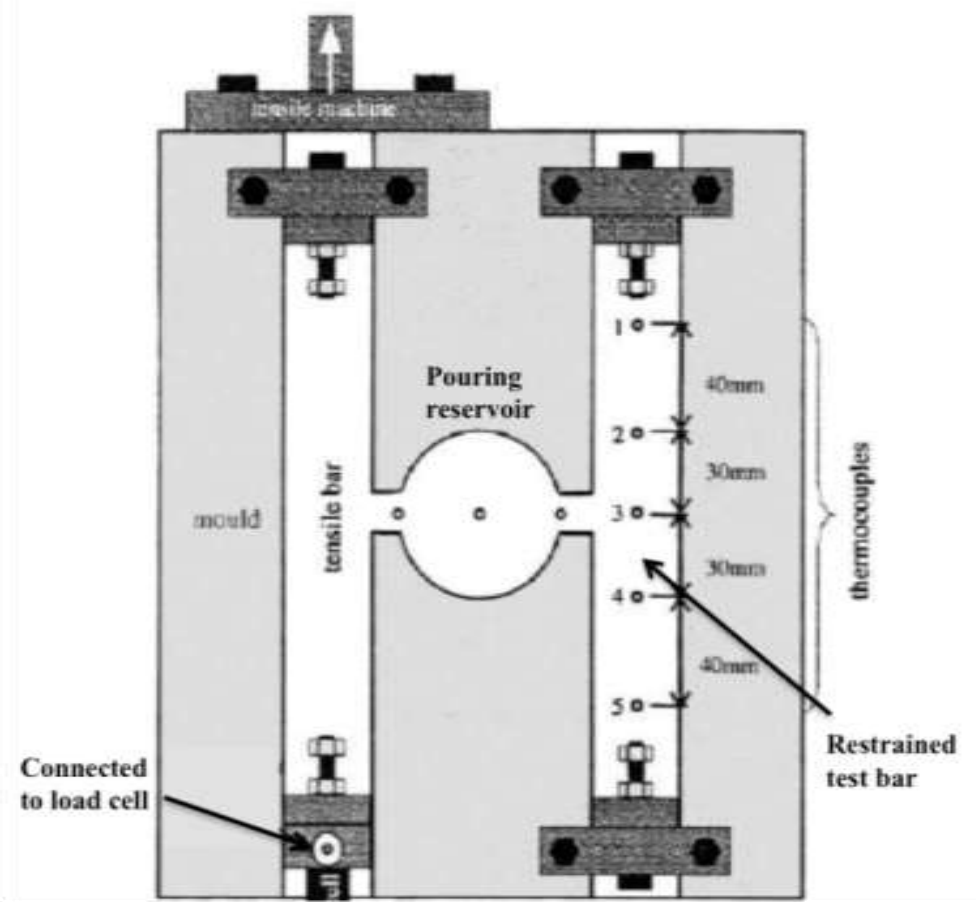

Figure 2-11. Hot tear apparatus developed by Instone et al. [49].

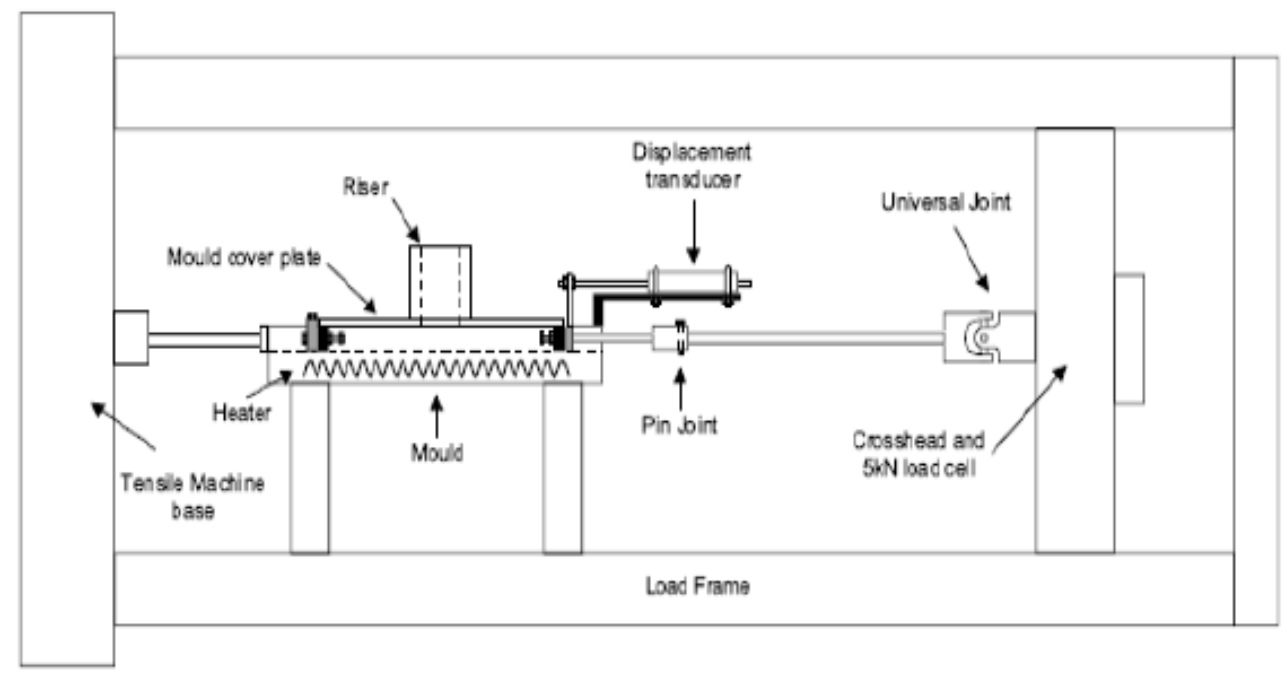

Figure 2-12. Schematic diagram of Instone's hot tear test rig [49].

More recently, Davidson et al. [50] modified the equipment developed by Instone et al. to allow direct observation of the hot spot region during solidification of an Al-0.5 wt\% Cu alloy. The mold was modified to incorporate a window above the hot spot region, which enabled observation of hot tear formation and growth. The visual observations were combined with load 
and temperature data and it was found that load development began at about $90 \%$ solid and a hot tear formed a short time later, at between $93 \%$ and $96 \%$ solid [50].

Cao et al. [51-53] developed an instrumented constrained rod-casting (CRC) mold to quantitatively analyze the hot tearing of $\mathrm{Mg}$ alloys. The experimental setup is shown in Figure 2-14. The mold's design was based on the dog-bone shape and consisted of six upper rods with varying lengths and a bottom rod directly connected to a load cell (via a threaded rod). A thermocouple was also placed along the bottom rod at the junction with the sprue (i.e. hot spot region). Therefore, the upper six rods were used to characterize the hot tearing susceptibility of the alloys while the bottom rod was used to collect load and temperature data and subsequently determine the onset of hot tearing. Upon pouring, the metal flowed into the mold and solidified onto the threaded rod attached to the load cell. As solidification progressed, the threaded rod restricted the free contraction of the casting rod, resulting in a buildup of contraction force. The contraction force was recorded in a plot against time and used in conjunction with the cooling curve generated from the thermocouple. An example of these curves is shown in Figure 2-15 for an AZ91E Mg alloy [51]. A sudden drop in force along the load curve was attributed to the formation of a hot tear and the temperature at which the drop occurred was deemed the onset temperature of hot tearing. In the case of the AZ91E alloy shown in Figure 2-15, the hot tear was found to occur at $499^{\circ} \mathrm{C}$ at a solid fraction of 0.77 [51].

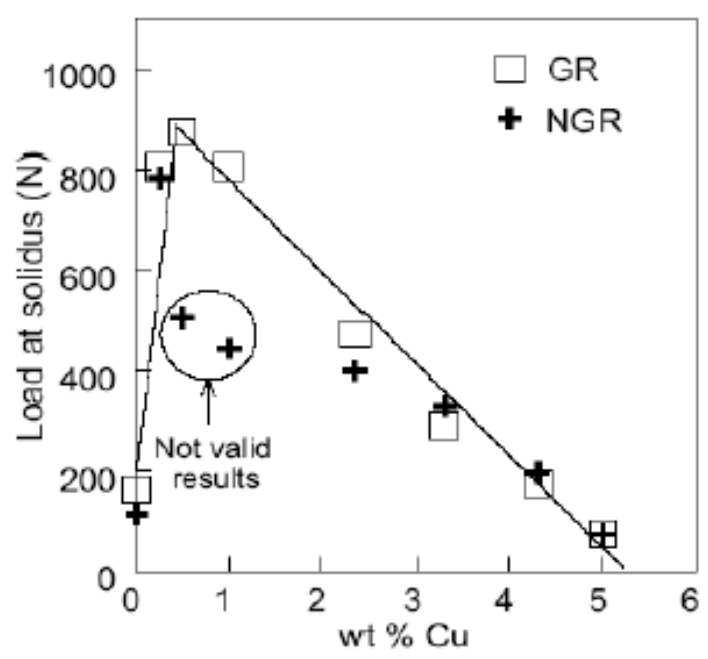

Figure 2-13. The measured load at solidus temperature for a series of Al-Cu alloys recorded by Viano et al. [37]. 


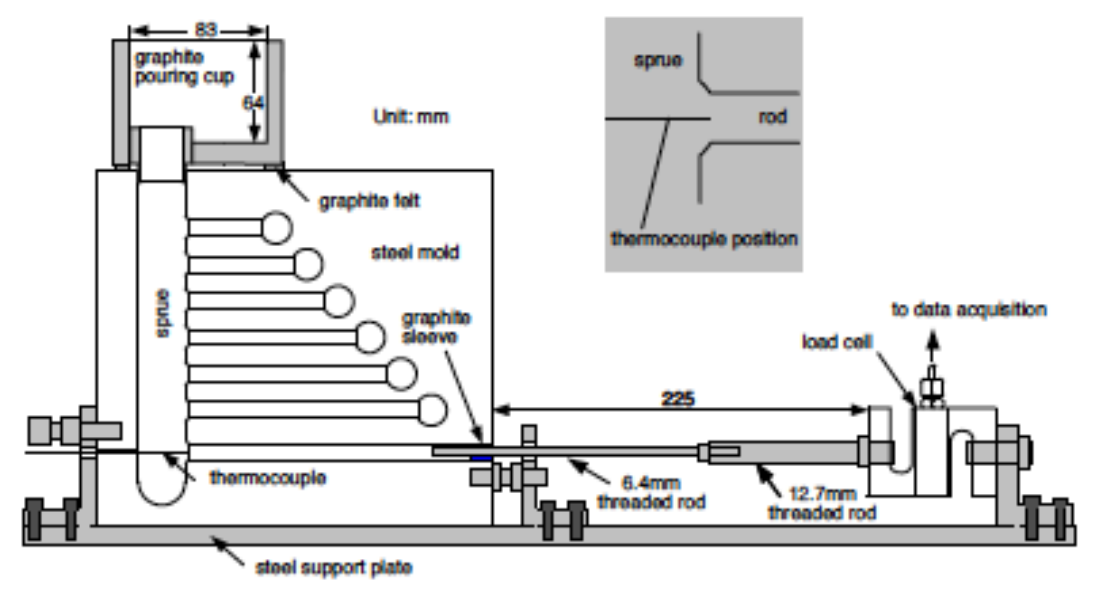

Figure 2-14. Instrumented CRC mold developed by Cao et al. [51].

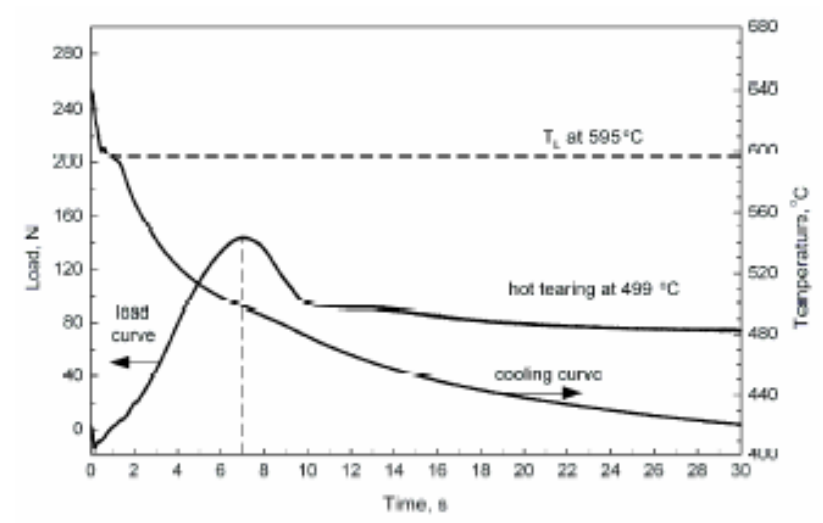

Figure 2-15. Load and cooling curve for an AZ91E Mg alloy [51].

Zhen et al. [46] developed a similar apparatus to measure the contraction stress induced by the solidification. It consisted of a CRC mold, a contraction force measurement system with a load cell, and a data acquisition system (Figure 2-16). Similar to the setup of Cao et al. [51-53], when a hot tear occurred during solidification, the contraction stress induced was released. This was manifested by a sudden drop on the force curve of the load cell. The behaviour of hot tearing could then be investigated by analyzing force development. Specifically, the initiation of hot tearing, evolution and final size of the hot tear and the onset temperature of hot tearing could be determined. One main and critical advantage relative to the setup of Cao et al. was attributed to the design of the casting rod. In particular, the diameter of the casting rod slightly decreased from the sprue end $(12.5 \mathrm{~mm})$ towards the opposite end (i.e. by the load cell) $(10 \mathrm{~mm})$. The slight taper in the casting rod reduces the influence of friction between the mold wall and casting rod. 
In turn, this helps to produce enhanced repeatability of results, as illustrated in past research using this apparatus $[31,32,46]$. More details on this apparatus are provided in Chapter 3 .

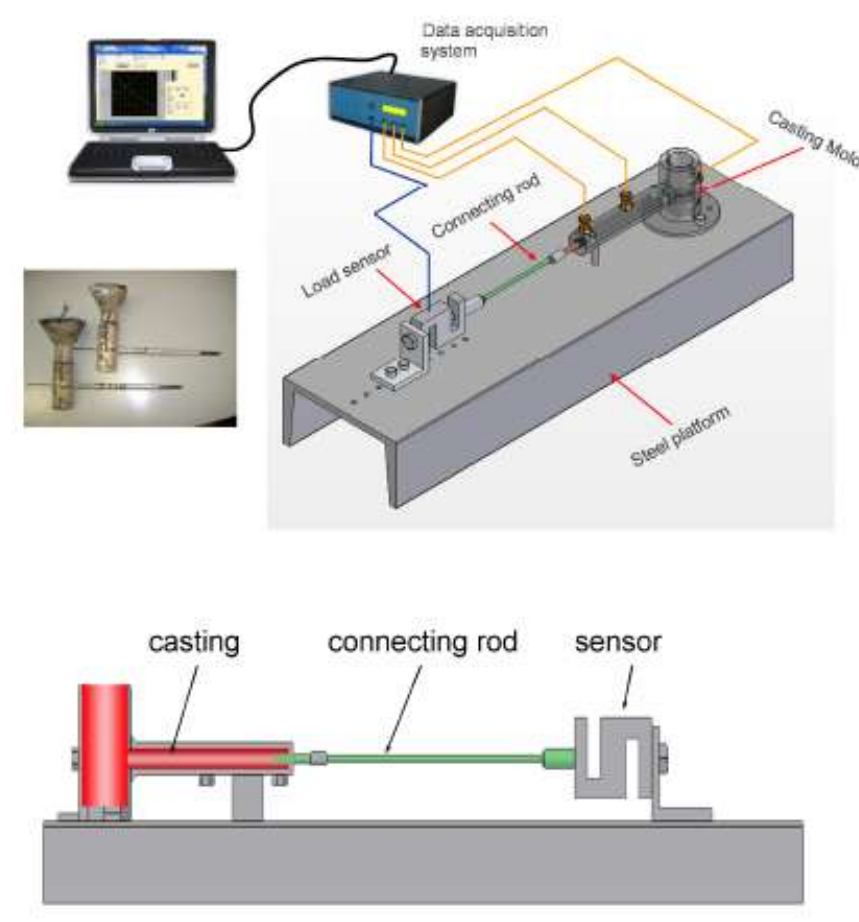

Figure 2-16. Hot tearing setup developed by Zhen et al. [46].

\subsection{Linear Variable Differential Transformer (LVDT) Method}

A technique for measuring linear contraction during solidification was used by Eskin et al. [39]. They carried out a systematic study on the hot tearing behaviour of binary Al alloys. The apparatus is shown in Figure 2-17. It consisted of a T-shaped graphite mold with a moving wall, a water-cooled bronze base and an LVDT attached to the moving wall and aligned along the longitudinal axis of the mold. A metallic threaded rod embedded into the moving wall enabled the solidifying melt to attach to the moving wall. The casting's contraction during solidification was measured by the LVDT (attached to the moving wall). Thermocouples were also inserted along the mold for temperature measurements (not shown in Figure 2-17). Data acquisition was used to simultaneously measure the linear contraction and temperature of the alloys. Thus, this enabled the determination of the onset temperature of thermal contraction and the total amount of contraction during solidification for various Al alloys [39]. The total amount of contraction 
accumulated in the solidification range was found to correlate well with the hot tearing susceptibility of the alloys. The effect of grain refinement on linear contraction and hot tearing was also determined (see Section 2.2.2). Similar equipment was developed by Li et al. [54] to measure the contraction of $\mathrm{Al}-\mathrm{Cu}$ alloy 206 with a relation to hot tearing.

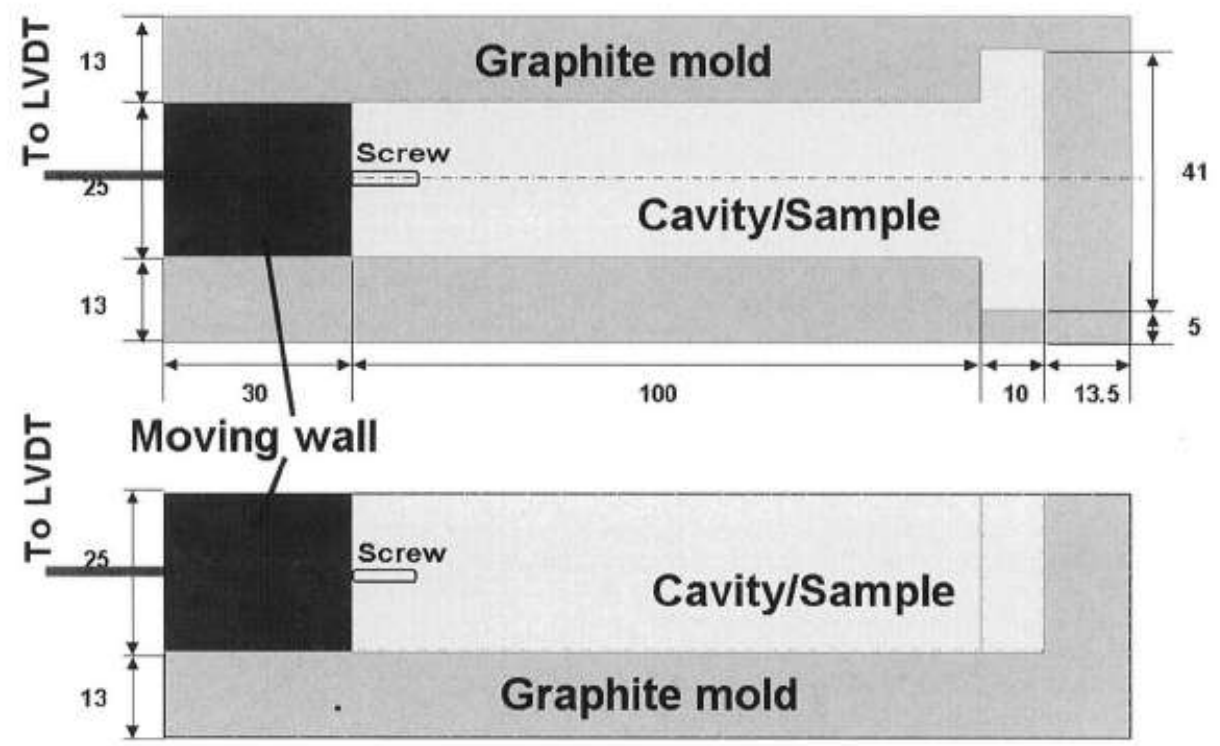

Figure 2-17. T-shaped mold equipped with moving wall and LVDT [39].

\subsubsection{Neutron Diffraction}

Neutron diffraction is a common technique used to determine the atomic or crystal structure of a material. The technique requires a source of neutrons either generated from a nuclear reactor or a spallation source. The advantage of neutron diffraction stems from the fact that neutrons are electrically neutral and unlike charged particles (i.e. electrons from X-rays), they are not scattered by the electron clouds of surrounding atoms [55]. This gives neutrons the capability to interact directly with the nuclei of atoms in a sample and penetrate deeply into the bulk of the sample material. The deep penetration of neutrons into materials enables the investigation of bulk samples in a variety of environments including furnaces and pressure vessels.

There are essentially two neutron diffraction techniques, namely, conventional $\theta / 2 \theta$ scanning, where a continuous beam of neutrons is released from a reactor source, and time of flight 
approaches, where pulsed beam of neutrons is produced from spallation sources [55]. In a nuclear spallation source, such as a synchroton accelerator, high-energy protons strike a target material and trigger the emission of neutrons [56]. In the case of a nuclear reactor source, neutrons are generated at various wavelengths in a fission process. Such "white" neutrons are then directed towards a spectrometer. An example of a typical triple axis spectrometer and its key components are shown in Figure 2-18 [57]. The key components of the spectrometer include the monochromator, the monochromator shielding drum, the incident and diffracted beam collimators and the diffracted beam analyzer.

The monochromator is a disc made of a single crystal material that is used to extract a single wavelength of neutrons from the beam of "white" neutrons. Any undesirable "white" neutron beam wavelengths and stray radiation from the nuclear reactor are absorbed by the monochromator shielding drum [57]. The monochromatic neutron beam exits the monochromator shielding drum via the incident beam collimator, and directly interacts with the sample, which lies on the sample stage. Once in contact with the sample, the incident beam will diffract if two conditions are met, namely, if the interplanar spacing of the sample is of the order of the wavelength of the neutron beam and if the Bragg condition is met. The Bragg condition states that in order for diffracted rays (or neutron beam) to emerge from a sample, the rays must be in phase with each other and result in constructive interference. Constructive interference is maintained if the path difference between two (or more) diffracted rays remain an integer multiple of the incident beam wavelength. The path difference resulting from constructive interference between adjacent rays is given by Bragg's law, shown in Equation 3, which relates the wavelength, $\lambda$, to interplanar spacing, $d_{h k l}$, and the scattering angle, $2 \theta$, for a given order of diffraction, $n$.

$$
n \lambda=2 d_{h k l} \sin \theta
$$

The diffracted neutron beam that emerges from the sample is then directed through the diffracted beam collimator towards the analyzer, as shown in Figure 2-18. Finally, the diffracted beam analyzer measures the angular position and intensity of diffracted neutrons. 
Bragg's law (Equation 3) describes the fundamental relationship between the neutron beam wavelength, interplanar spacing and the angle of diffracted neutrons. From the generated monochromatic wavelength, at a given order of diffraction (usually first order) and a predetermined Bragg angle, the interplanar spacing can be determined. The determination of interplanar spacing is a fundamental principle of elastic residual stress measurement in crystalline materials [57].

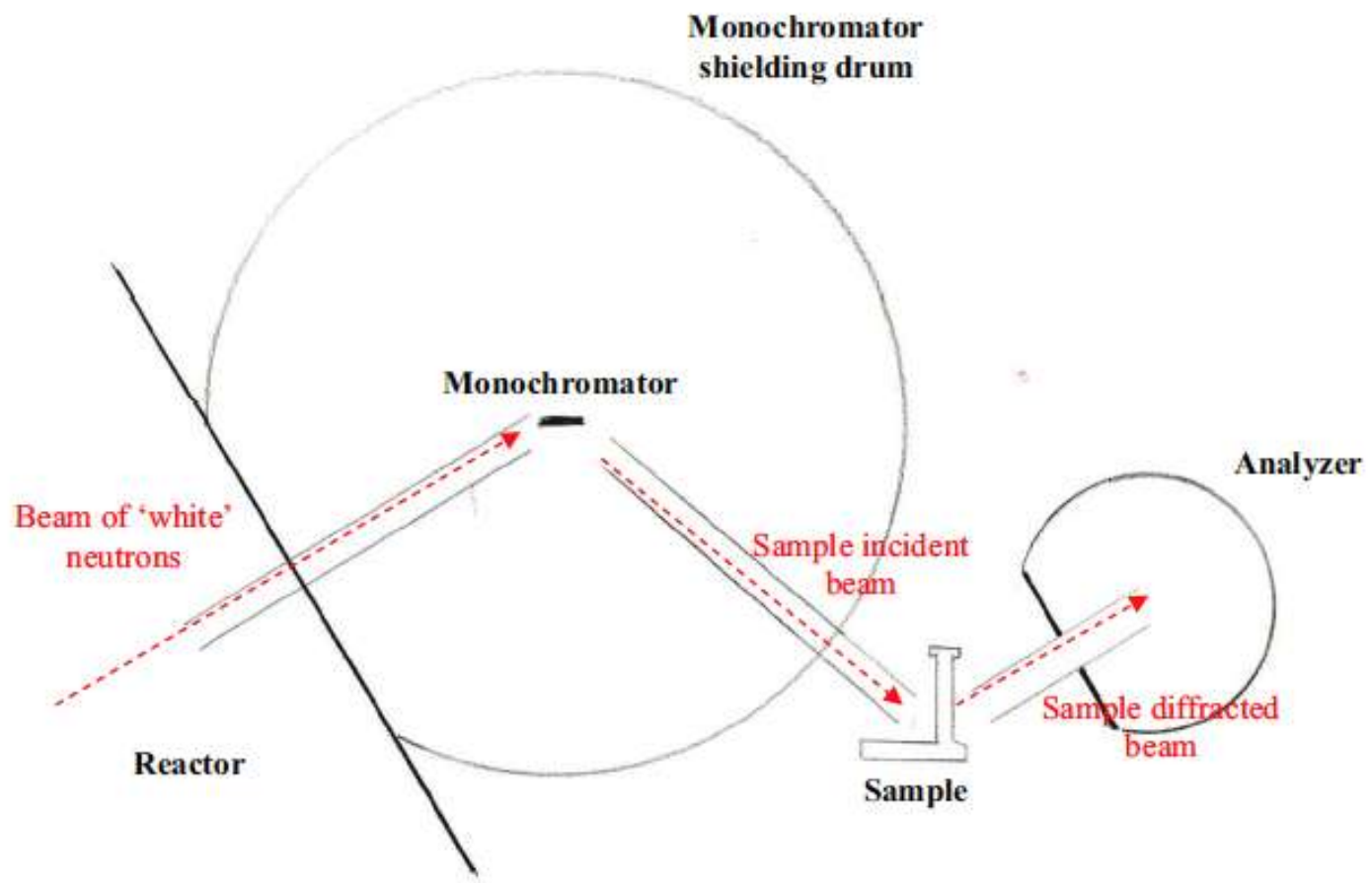

Figure 2-18. Schematic illustration of a triple axis spectrometer [57].

\subsection{Measurement of Residual Strain and Stress by Neutron Diffraction}

Residual stresses in a body are those that are not necessary to maintain equilibrium between the body and its surroundings [58,59]. Hence, across any section through the body the component of the residual stress normal to the section must balance. The length over which they do balance in any direction is known as the characteristic length, $l_{o}[60]$. Residual stresses can be classified into three categories according to characteristic length scales [58-60]. Stresses that neglect the underlying microstructure are defined as type I stresses. These stresses are also known as macrostresses since they equilibrate over macroscopic dimensions, such as the scale of the 
structure. Type II stresses are microstructurally related and equilibrate over a scale of a few grain diameters. These stresses are also termed intergranular stresses and tend to arise from inhomogeneity at the grain scale, such as from differences in slip behaviour from grain to grain [60]. Finally, type III stresses are microstresses at an even finer scale (than type II stresses). Such stresses arise from heterogeneous behaviour at the atomic scale, such as from dislocations or point defects, and therefore, equilibrate over small atomic distances.

Residual stresses are introduced into engineering components through a variety of methods including, machining, heat treatment, forging, welding and carburizing [60]. In casting applications, these stresses arise from thermal gradients resulting from non-uniform cooling. For instance, during die-casting, the cold mold wall triggers rapid solidification along the exterior of the casting, while the mold's warmer interior results in slower solidification of the casting interior. As a result, the casting's warm interior hinders the free contraction of the colder casting exterior and generates thermal stresses within the casting. Further, varying thicknesses along the casting cavity can alter the cooling rates at different regions along the mold and further contribute to the build up of stress. This can ultimately lead to plastic deformation and subsequent failure of the casting. Thus, the measurement of such stresses can significantly contribute to quantifying the hot tearing behaviour of an alloy.

Neutron diffraction is a non-destructive technique used to measure residual strain and stress in the bulk of a sample. The method utilizes Bragg's law to determine the interplanar spacing, $d_{h k l}$, for a given wavelength and diffraction angle. When a material is subjected to a tensile load, $d_{h k l}$ increases in the tensile loading direction with respect to its stress-free value $d_{o-h k l}$. Similarly, the interplanar spacing decreases for compressive loads. The stress-free value is determined from a stress-relieved (i.e. heat-treated or machined) sample. The strain, $\varepsilon_{h k l}$, experienced by the material can be expressed using the peak-shift method, as shown in Equation 4:

$$
\varepsilon_{h k l}=\left(d_{h k l}-d_{o-h k l}\right) / d_{o-h k l}
$$

For an isotropic material, the relationship between stress and strain in the orthogonal Cartesian $(\mathrm{x}, \mathrm{y}$ and $\mathrm{z}$ ) coordinate system is given by the generalized form of Hooke's law (Equation 5): 


$$
\sigma_{\alpha}=\mathrm{E} /(1+v)\left\{\varepsilon_{\alpha}+[v /(1-2 v)]\left(\varepsilon_{x}+\varepsilon_{y}+\varepsilon_{z}\right)\right\} \quad \alpha=(\mathrm{x}, \mathrm{y}, \mathrm{z})
$$

Where:

$$
\begin{aligned}
\sigma_{x}, \sigma_{y}, \sigma_{z} & =\text { stress in } x, y \text { and } z \text {-direction, respectively } \\
\varepsilon_{x}, \varepsilon_{y}, \varepsilon_{z} & =\text { strain in } x, y \text { and } z \text {-direction, respectively } \\
v & =\text { Poisson's ratio } \\
\mathrm{E} & =\text { Young's modulus of elasticity }
\end{aligned}
$$

\subsection{Analysis of Hot Tearing by Neutron Diffraction}

Neutron diffraction was successfully used in recent studies to relate residual strain to hot tearing. Bichler et al. [57,61,62] carried out a systematic investigation on the relationship between residual strain (and stress) on the hot tearing behaviour of Mg alloys AZ91D and AE42. The authors felt that neutron diffraction was advantageous over other quantitative methods, since neutron diffraction does not require the use of measurement probes (e.g. load cells and LVDTs) that can interfere with the natural solidification of the alloy and generate unreliable results [57]. In their study, residual strain and hot tearing susceptibility were found to be particularly dependant on solidification rate. High solidification rates did not enable enough time for liquid metal to alleviate casting strain and therefore, resulted in hot tear formation. In contrast, lower solidification rates caused liquid metal feeding particularly through interdendritic regions, thus successfully alleviating casting strain and eliminating hot tears.

\section{$\underline{\text { Section Summary }}$}

Various methods used to characterize hot tearing susceptibility were described. The ring mold test, "dog-bone" mold test and cold finger test are advantageous because of their simple designs, which make them easy to use. However, their inability to quantitatively assess hot tearing is a limiting factor. As such, methods utilizing load cells and LVDTs to respectively measure contraction force (stress) and contraction during solidification were developed. These techniques provide a more quantitative approach. Lastly, a less intrusive approach (i.e. no use of measurement probes) of using neutron diffraction to measure residual stresses with a direct 
correlation to hot tearing was reviewed. This approach has shown promise in quantifying hot tearing in $\mathrm{Mg}$ alloys and is therefore considered for use in other alloys.

\section{Chapter Summary}

The literature review suggests that hot tearing is a complex phenomenon. The theories of hot tearing postulate that stress, strain, strain-rate and alloy microstructure are all underlying factors. Further, various factors including alloy composition, grain size and morphology and casting process parameters are seen to influence hot tear formation. However, quantification of stress and strain generated during casting is lacking in many hot tearing investigations. Moreover, since hot tearing is a solidification phenomenon, an understanding of microstructure development during solidification will help to paint a clearer picture of the hot tearing mechanisms. With this consideration, the current research extends the use of neutron diffraction for residual strain and stress measurements to the B206 aluminum alloy. Specifically, the effect of grain refinement on hot tearing and subsequent residual strain is investigated. Further, the load cell method is used to determine the onset temperature of hot tearing in B206 and finally, a unique approach using in situ neutron diffraction to characterize the solidification kinetics of B206 is implemented. The following chapter describes the methodology undertaken in this research. 


\section{Chapter 3 - Experimental Procedure}

The experimental methodology carried out in this research is outlined in this chapter. Details on the casting parameters used for the ex situ and in situ experiments are given. Further, methods undertaken during the neutron diffraction experiments are described. Finally, the chapter concludes with a description of the various methods of microscopy used in this research.

\subsection{Ex Situ Casting Experiments}

Ex situ permanent mold casting experiments were carried out at the Centre for Near-net-shape Processing of Materials (CNPM) at Ryerson University. This section describes the methodology used for the experiments. Specifically, the mold design and setup are described and the alloy composition and casting parameters are presented.

\subsubsection{Permanent Mold Design}

The permanent mold used in this research is shown in Figures 3-1 and 3-2. The mold geometry was intentionally designed to promote hot tear formation in the castings. Its design was based on the "dog-bone" mold design from literature (Section 2.2.3.2). The mold was machined from a block of H-13 steel. The casting cavity consisted of a $180 \mathrm{~mm}$ downsprue and a $260 \mathrm{~mm}$ long horizontal bar with an end restraint. The casting thickness was $20 \mathrm{~mm}$. The $90^{\circ}$ junction between the downsprue and the horizontal bar, in conjunction with the end restraint, restricted the horizontal bar from contracting freely during solidification. As a result, a hot tear formed along the horizontal bar.

The mold temperature was measured using three K-type thermocouples inserted in the bottom face of the mold, as shown in Figure 3-1. These thermocouples were attached to a Daytronic System 10 data acquisition unit, which monitored the mold temperature during the casting experiments. The average temperature reading of the three thermocouples was taken as the average mold temperature. Prior to each casting, the desired mold temperature was pre-set and the mold was homogenized at this temperature for a minimum of 10 minutes. 


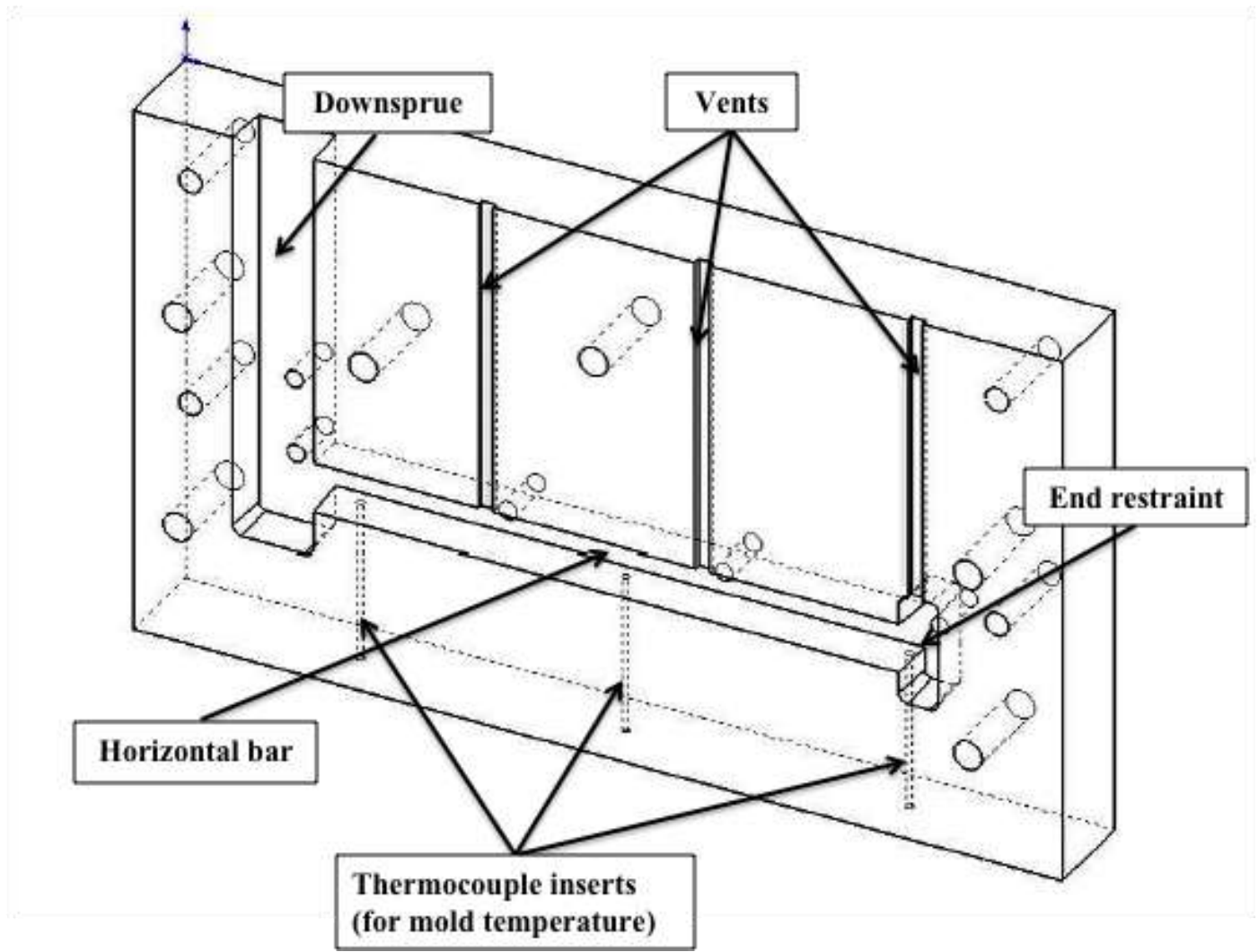

Figure 3-1. H-13 steel permanent mold (isometric view).

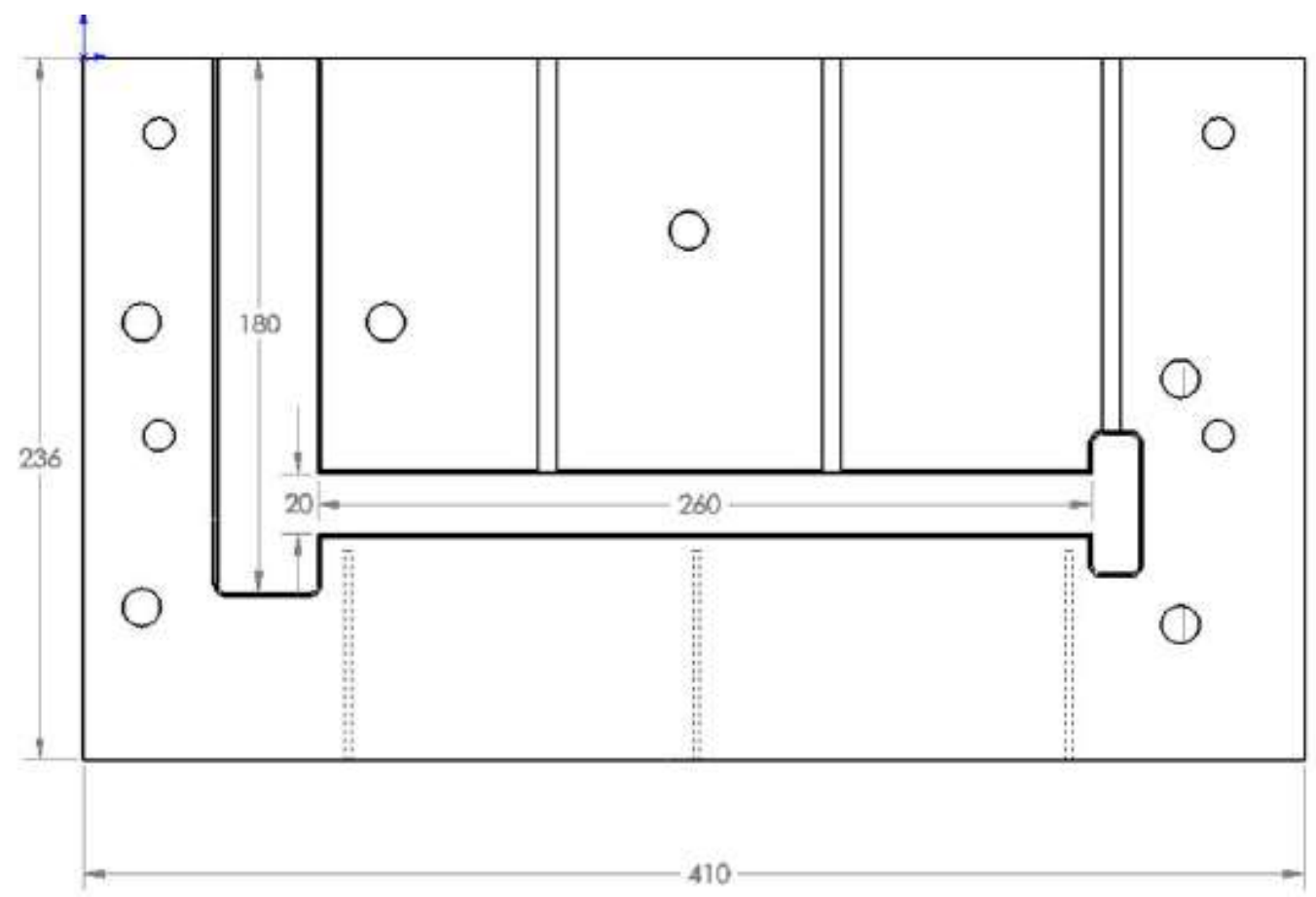

Figure 3-2. Dimensions of permanent mold (in mm). 
The mold was also equipped with three vents, as shown in Figure 3-1. The vents ensured that no gases remained entrapped in the casting cavity during casting, and that a sound casting was therefore produced.

\subsubsection{Mold Mounting}

The mold was mounted on a Cooper Chapman pneumatic sand core-making machine. The experimental set-up is displayed in Figure 3-3.

Four bolts (12.5 $\mathrm{mm}$ diameter) were used to attach the permanent mold to the core-making machine. Two sliding bolts were $20 \mathrm{~cm}$ long and were inserted $2 \mathrm{~cm}$ into the mold. The remainder of the mounting bolts extended $2 \mathrm{~cm}$ beyond the spacer plates, as shown in Figure 3-2 thus allowing the permanent mold to slide and pivot.

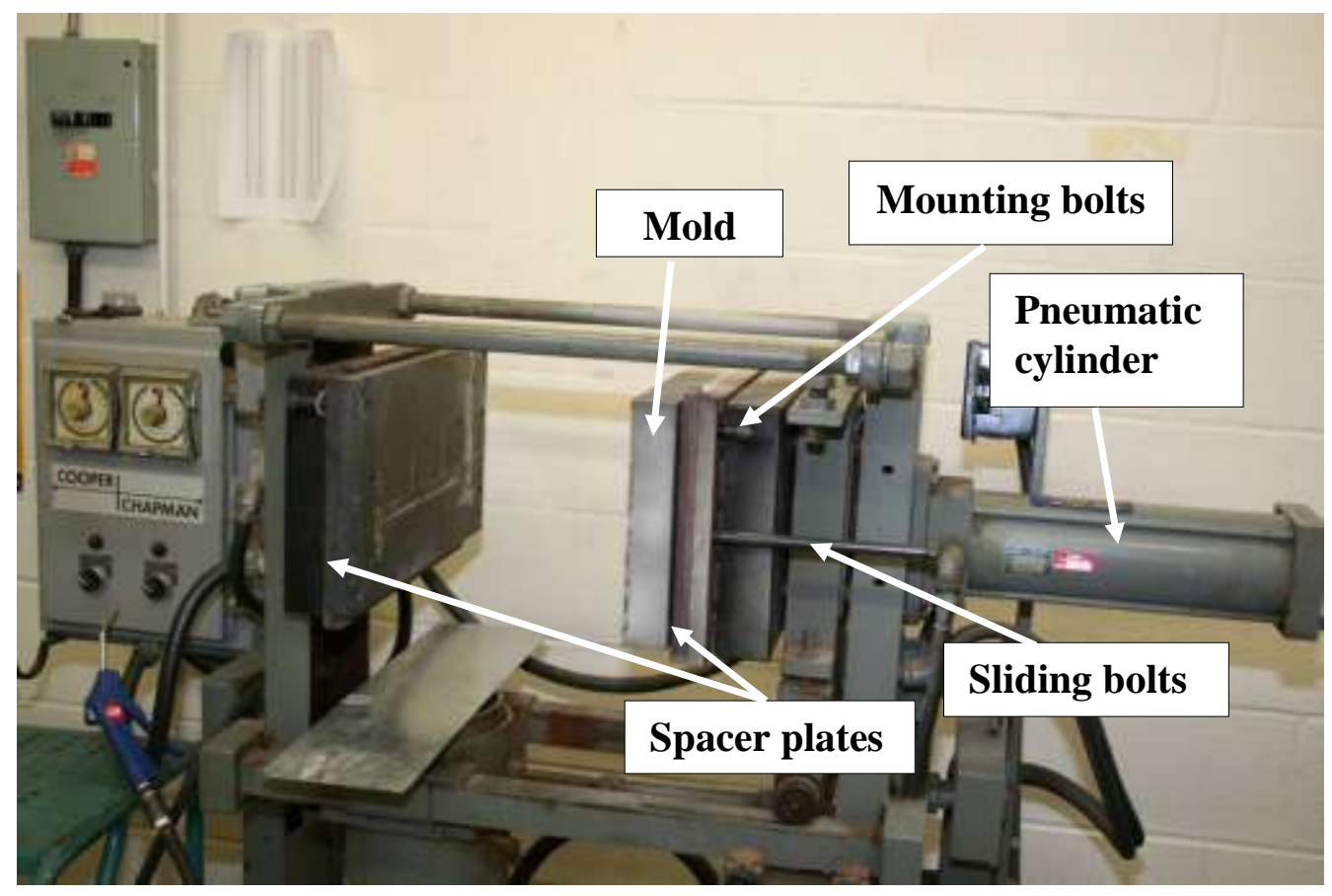

Figure 3-3. Cooper-Chapman pneumatic sand core-making machine with mounted mold.

The machine was equipped with electric resistance strip heaters and a thermostat, which enabled temperature control of the mold (up to $400^{\circ} \mathrm{C}$ ). The Cooper-Chapman press was also connected 
to a compressor airline, which powered the pneumatic cylinder, which opened and closed the permanent mold.

Prior to each pour, the mold was opened and five ejector pins ( $7.9 \mathrm{~mm}$ diameter) were inserted into the five ejector pin holes located along the casting cavity. The pin heads were placed flush with the casting cavity wall during casting, to ensure that the pins did not interfere with metal flow. The lengths of the pins were slightly $(\sim 5 \mathrm{~mm})$ shorter than the thickness of the mold. As a result, a small gap remained between the end of the ejector pin and the back of the mold wall.

Once a casting was produced, the mold was opened and pushed along the sliding bolts to create a $2.5 \mathrm{~cm}$ wide gap between the back wall of the mold and the spacer plate located behind the mold. A second set of ejector pins was inserted into the ejector pin holes from the back. This second set of ejector pins was protruding out of the back side of the mold. The mold was then closed and the second set of ejector pins pushed on the first set of ejector pins, thereby pushing out the casting. Care was taken to ensure that casting ejection was uniform and casting distortion during ejection was avoided.

\subsubsection{Mold Coating}

Boron-nitride (BN) coating, supplied by ZYP Chemicals, was applied to the mold, in order to avoid erosion of the $\mathrm{H}-13$ mold by liquid aluminum. The mold was preheated to $150{ }^{\circ} \mathrm{C}$ prior to applying the $\mathrm{BN}$ coating with a low-pressure spray gun. The $\mathrm{BN}$ coating was then allowed to cure on the mold for 24 hours prior to casting. The coating was removed and reapplied for each casting experiment.

\subsubsection{Control of Casting Temperature}

Three K-type thermocouples were inserted into machined slots in the mold's casting cavity, in order to collect real-time temperature data of the solidifying casting. The thermocouples were placed at the locations shown in Figure 3-4. Specifically, one thermocouple was inserted by the downsprue, one at the middle of the horizontal bar and one by the end restraint. Machined steel 
inserts were placed in the mold slots to hold the thermocouples in place. Temperature measurements were used to monitor cooling rate, solidification time and freezing range.

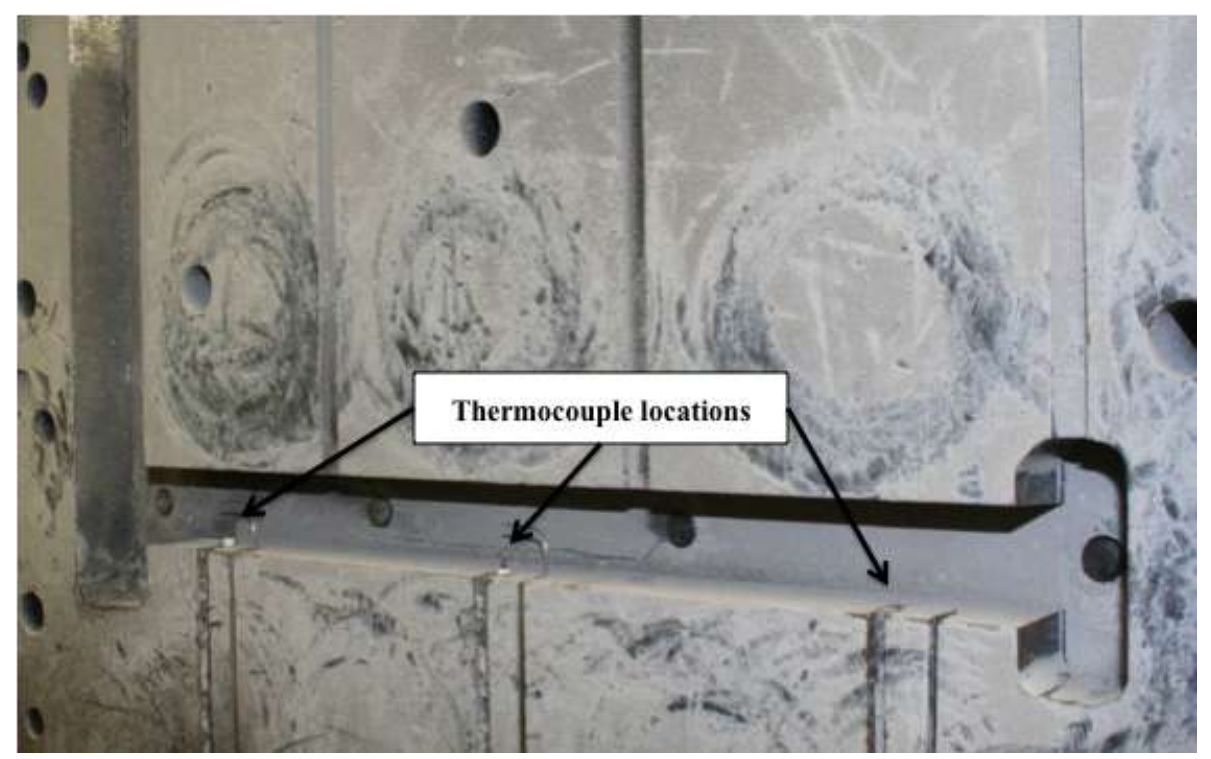

Figure 3-4. Location of thermocouples along casting cavity.

The thermocouples were connected to a Daytronic System 10 data acquisition unit. The data acquisition system was calibrated with a Fluke 714 temperature calibrator prior to use. Also, each thermocouple was tested to ensure proper function before being inserted into the casting cavity. For each experiment, the temperature data was collected for a total of 360 seconds at a sampling rate of seven readings / second. The accuracy of the thermocouples was $\pm 0.5^{\circ} \mathrm{C}$.

\subsubsection{Alloy Melting and Casting}

The B206 aluminum alloy used in this research was obtained as ingots from Alcan Inc. The alloy composition as determined from an emission spectrometer is presented in Table 3-1.

Table 3-1. B206 alloy composition, in wt\%.

\begin{tabular}{|c|c|c|c|c|c|c|c|c|c|}
\hline $\mathbf{C u}$ & $\mathbf{M n}$ & $\mathbf{M g}$ & $\mathbf{F e}$ & $\mathbf{S i}$ & $\mathbf{N i}$ & $\mathbf{Z n}$ & $\mathbf{S n}$ & $\mathbf{T i}$ & $\mathbf{A l}$ \\
\hline 4.9 & 0.38 & 0.24 & 0.05 & 0.04 & $<0.01$ & $<0.01$ & $<0.01$ & $<0.01$ & Bal. \\
\hline
\end{tabular}


The B206 alloy was grain refined using Al-5Ti-1B Tibor $^{\circledR}$ master alloy, whose composition is given in Table 3-2. The alloy grain size was manipulated by varying the amount of titanium. Three addition levels were investigated, namely: unrefined, $0.02 \mathrm{wt} \% \mathrm{Ti}$ and $0.05 \mathrm{wt} \% \mathrm{Ti}$. Actual composition analysis using gas emission spectroscopy was carried out on the castings at Gamma Foundries in Richmond Hill, Ontario. The results are presented in Appendix 1 (Table A.1-1).

Table 3-2. Al-5Ti-1B master alloy composition, in wt\%.

\begin{tabular}{|c|c|c|c|c|c|c|}
\hline $\mathbf{A l}$ & $\mathbf{T i}$ & $\mathbf{B}$ & $\mathbf{F e}$ & $\mathbf{V}$ & $\mathbf{S i}$ & $\mathbf{Z n}$ \\
\hline 93.64 & 5.0 & 1.0 & 0.1 & 0.1 & 0.06 & 0.01 \\
\hline
\end{tabular}

The B206 alloy was melted in an electric resistance furnace. Virgin ingots were used for each casting trial (i.e. no recycled alloy was used). Approximately $1 \mathrm{~kg}$ of the alloy was melted in a silicon-carbide crucible. The alloy was degassed at $760{ }^{\circ} \mathrm{C}$ using $0.25 \mathrm{wt} \%$ sodium fluorosilicate. In the case of the Ti-refined alloys, the master alloy was added to the melt at $760{ }^{\circ} \mathrm{C}$, prior to adding the degasser. The melt was then held for five minutes before being mechanically stirred for one minute. Stirring of the melt ensured that the master alloy was uniformly distributed in the melt. It was not required to stir the melt when pouring the unrefined alloy. Finally, the melt was skimmed and poured at $720^{\circ} \mathrm{C}\left(70{ }^{\circ} \mathrm{C}\right.$ superheat $)$.

The mold temperature was held constant at $380{ }^{\circ} \mathrm{C}$ for all ex situ casting experiments. This temperature was determined from preliminary trials. Section 2.2.2.3.2 demonstrated the importance of mold temperature on hot tearing. Lower mold temperatures (below $380{ }^{\circ} \mathrm{C}$ ) resulted in hot tears severe enough to fracture the casting. Such castings were not desired, as they could not be used for neutron diffraction strain mapping. As a result, the mold temperature was increased to the point in which hot tears formed but the casting remained intact. This was achieved only at $380{ }^{\circ} \mathrm{C}$. The experimental methodology for the neutron diffraction scans is discussed next. 


\subsection{Neutron Diffraction Strain Mapping Experiments}

Neutron diffraction strain mapping was carried out at the Canadian Neutron Beam Centre (CNBC) in Chalk River, Ontario. The E-3 beamline with a triple axis spectrometer was used for all residual strain measurements. The details of the neutron diffraction experiments including the sample setup, the selection of crystallographic planes and the experimental parameters used are described.

\subsubsection{Spectrometer and Sample Alignment}

The triple axis spectrometer used at CNBC is shown in Figure 3-5. The function of each component was described in Section 2.2.3.5 (see Figure 2-18). Prior to carrying out the strain mapping scans, the spectrometer was aligned with the help of the CNBC staff.

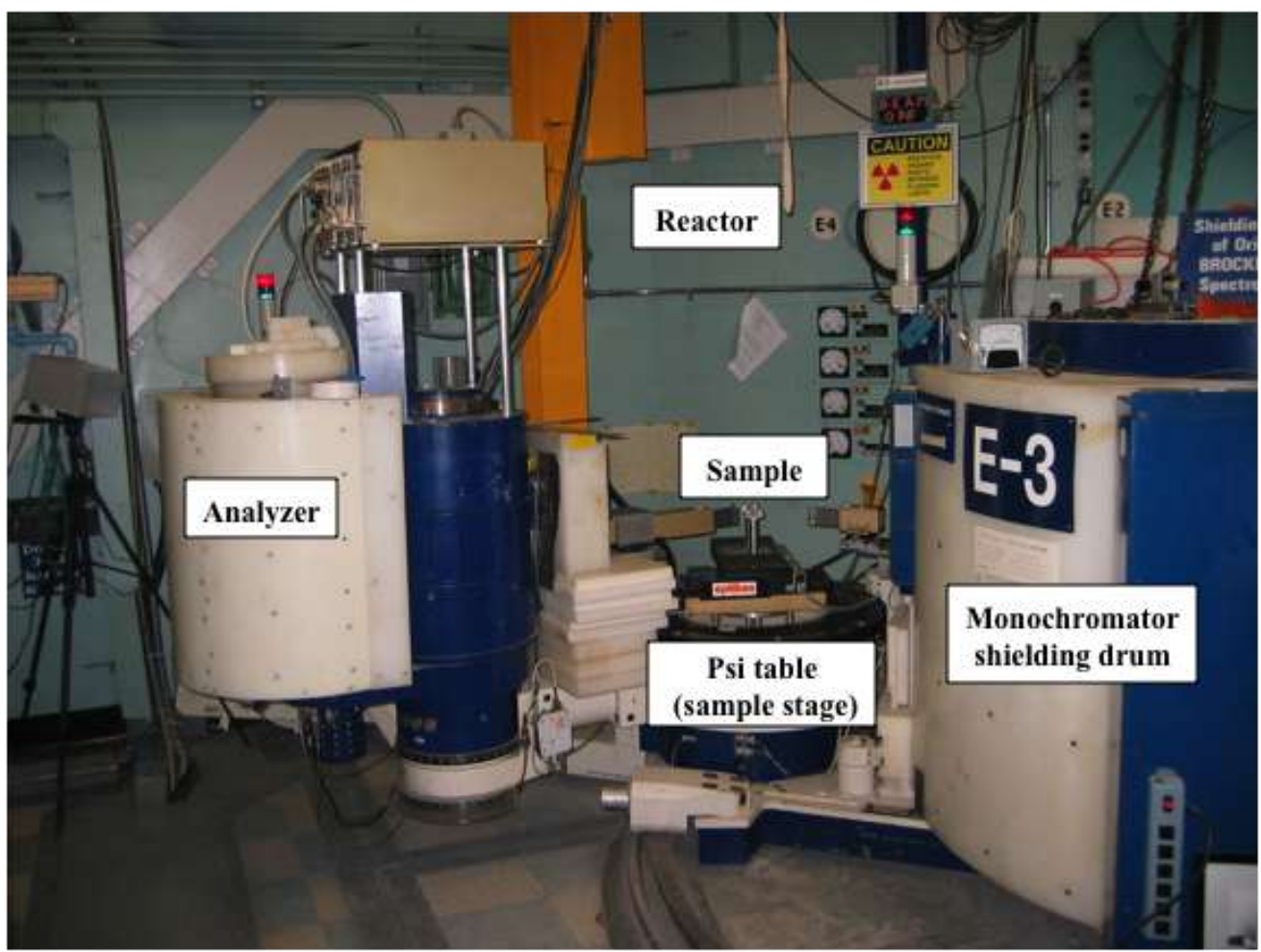

Figure 3-5. Triple axis spectrometer in Chalk River [57]. 
A Germanium (Ge) single-crystal monochromator was used to generate the incident neutron beam wavelength. By rotating the monochromator with respect to the beam of "white" neutrons (recall Section 2.2.3.5), various wavelengths could be generated. In this research, two wavelengths were used. Initially, a wavelength of $\lambda=2.74 \AA$ (Ge [113] monochromator) was used. Such a high wavelength enabled deep penetration of the beam into the bulk sample. However, the wavelength was then decreased to $\lambda=1.4 \AA$ (Ge [ $\left[\begin{array}{lll}\overline{1} & \overline{1} & 5\end{array}\right]$ monochromator) to provide a higher intensity beam and therefore, increase the amount of experiments that could be completed within the allotted time.

Wavelength calibration tests were carried out to determine the exact wavelength of the neutron incident beam. In these tests, ultra-high purity silicon ( $\mathrm{Si}$ ) was used. The interplanar spacing, $d_{h k l}$, of the Si and the Bragg diffraction angle (for a given crystallographic reflection) was known. Therefore, Bragg's Law (Equation 3) could be used to determine the actual wavelength of the incident neutron beam. The wavelength was measured for a minimum of three crystallographic reflections and the average value was used for strain measurements.

Residual strain measurements were carried out on individual B206 castings mounted on the sample stage of the spectrometer, as shown in Figure 3-6. The strain was measured in three principal directions (i.e. $x, y$ and $z$ ). The coordinate axes of the spectrometer relative to the casting geometry are shown in Figure 3-7. All experimental data in this thesis are presented relative to this coordinate axis.

Residual strain is measured in the direction parallel to the bisector of the incident and diffracted neutron beams, as shown in Figure 3-6. This bisector is known as the scattering vector. By shifting the sample with relation to the scattering vector, a strain map can be generated along the sample for a given orientation (e.g. $x, y, z$ etc.). Further, orientating the sample at various Bragg angles relative to the incident beam generates a strain map along various crystallographic plane orientations. In this research, the strain mapping was completed for the (111) and (311) crystallographic reflections for B206. These reflections were selected because of their strong diffraction intensity, which resulted in a high peak-to-background ratio. Further, both reflections are recommended for use in strain analysis for FCC metals (e.g. aluminum) because they are less 
sensitive to intergranular strain and their elastic response correlates well with the response of the bulk of the material [63]. Moreover, such reflections (more so the (311)) enabled a rectangular neutron beam sampling volume. A rectangular sampling volume is desirable because it ensures that the diffracted neutrons reaching the detector are ones that were diffracted only from the area of interest. The shaping of the neutron beam sampling volume is discussed in the following section.
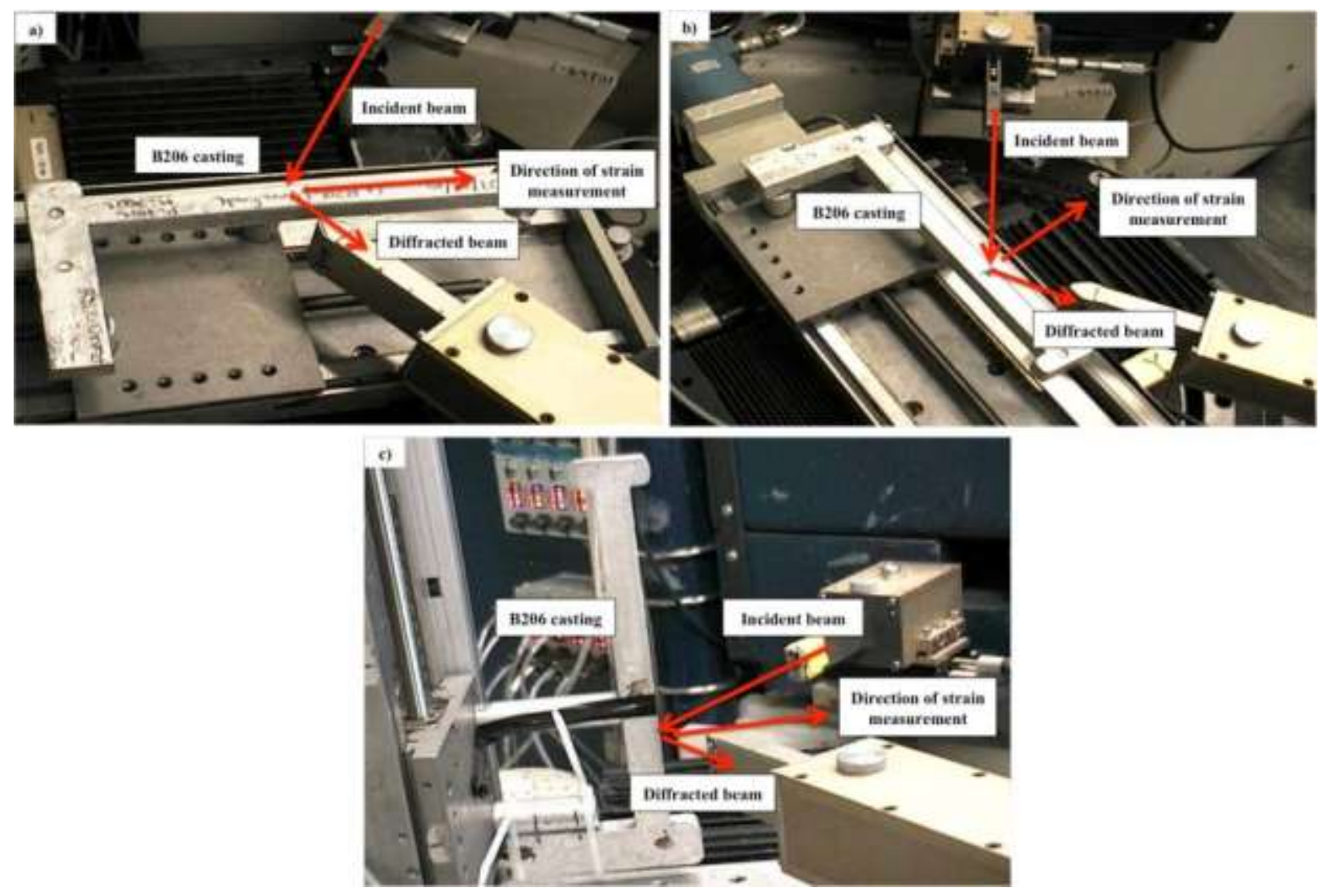

Figure 3-6. Sample setup for neutron diffraction strain mapping experiments for the a) $x$-direction scan, b) $y$-direction scan and c) $z$-direction scan.

\subsubsection{Neutron Beam Sampling Volume}

The beam of "white" neutrons (see Figure 2-18) generated from the reactor was directed towards the monochromator through a $5 \mathrm{x} \mathrm{cm}$ beam collimator. Upon colliding with the monochromator, the diffracted beam was then directed towards the sample through the $5 \mathrm{x} \mathrm{cm}$ incident beam collimator. 
Residual strain was measured in the sample at the volume defined by the intersection of the incident and diffracted neutron beam, known as the sampling volume (Figure 3-8). The sampling volume was shaped by beam width limiter slits that were installed in the incident and diffracted beam collimators. In this research, the beam width was limited to $4 \mathrm{~mm}$, as shown in Figure 3-8. The beam height was $20 \mathrm{~mm}$. In the case of the residual strain measurements in the $z$-direction, however, the beam height was reduced from $20 \mathrm{~mm}$ to $4 \mathrm{~mm}$ using beam height limiters.

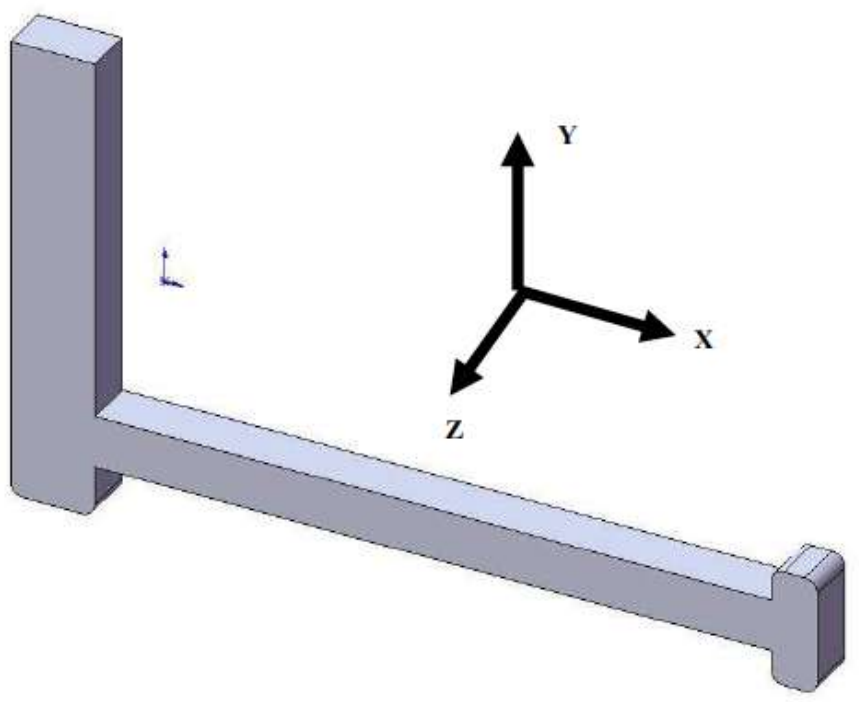

Figure 3-7. Coordinate axes of spectrometer in relation to casting geometry [57].

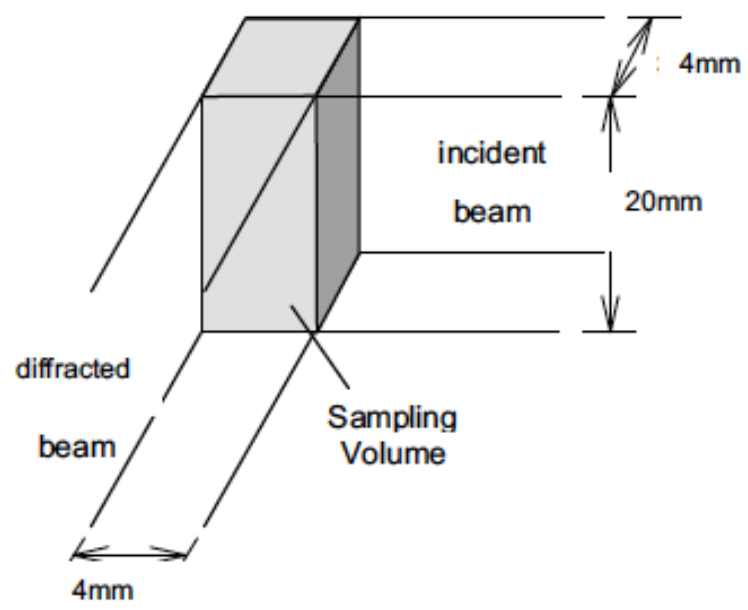

Figure 3-8. Neutron beam sampling volume [57]. 


\subsubsection{Selection of Samples for Strain Analysis}

Strain measurements were performed on three castings of the B206 alloy: one casting with an unrefined alloy, one casting containing $0.02 \mathrm{wt} \% \mathrm{Ti}$ and one casting containing $0.05 \mathrm{wt} \% \mathrm{Ti}$. These corresponded to a casting with a full hot tear, a casting with a small hairline fracture and a casting with no hot tear, respectively. Images of these castings are provided in Section 4.2. Strain measurements were carried out on the castings once solidification was complete and the samples were removed from the mold. As a result, the residual strain measurements were ex situ.

The location of the linescans where strain measurements were obtained is shown in Figure 3-9. The linescans were carried out along three locations of the horizontal bar: $4 \mathrm{~mm}$ below the top edge (i.e. top edge scan), along the centre (i.e. centreline scan) and $4 \mathrm{~mm}$ above the bottom edge (i.e. bottom edge scan). Further, a scan along the downsprue (4 $\mathrm{mm}$ from the edge closest to the horizontal bar) was carried out.

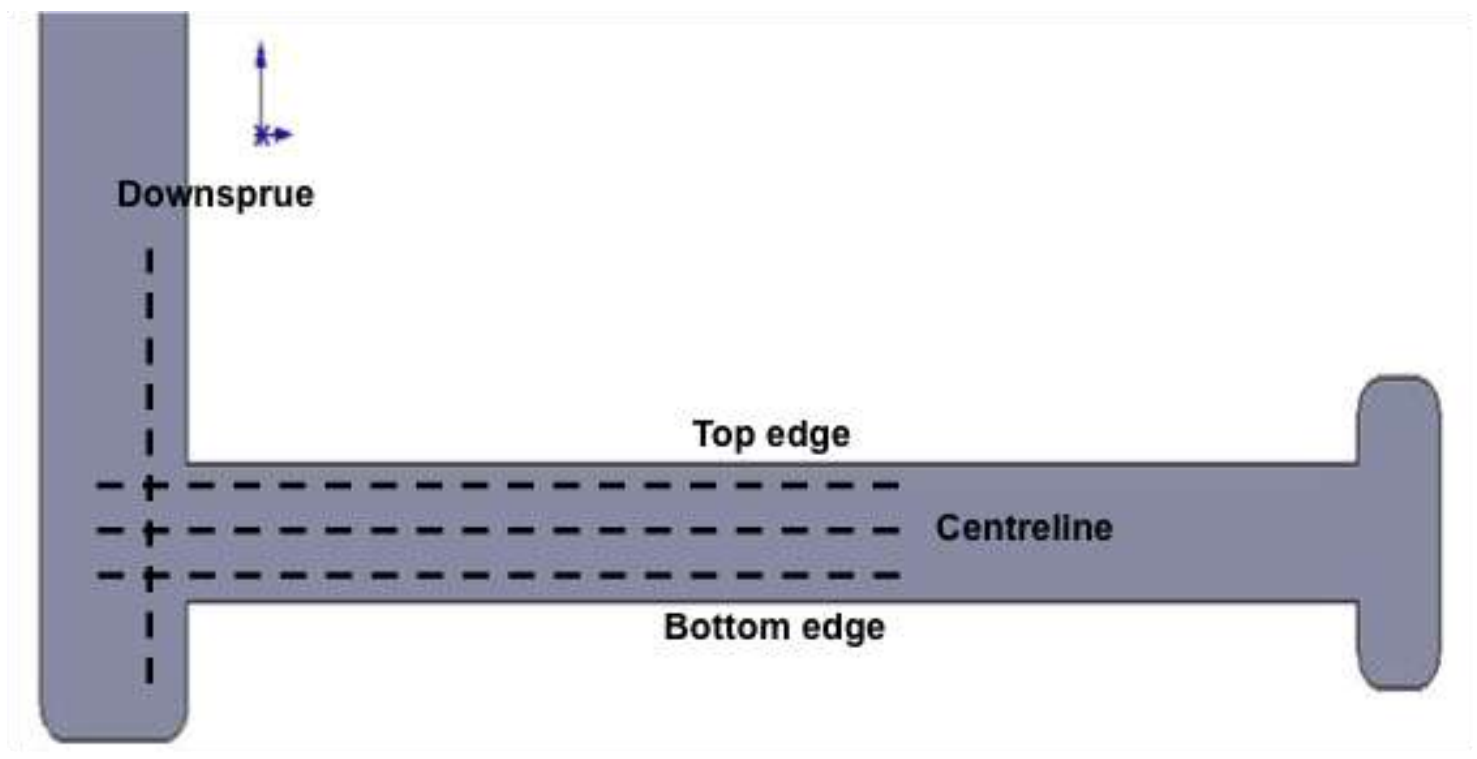

Figure 3-9. Location of linescans along B206 castings.

The length along the horizontal bar upon which the strain mapping scans were carried out is shown in Figure 3-10. The scans were initiated at a minimum of $10 \mathrm{~mm}$ into the downsprue portion of the horizontal bar, as shown in Figure 3-10. The alloys were then scanned up to 160 $\mathrm{mm}$ along the horizontal bar. 


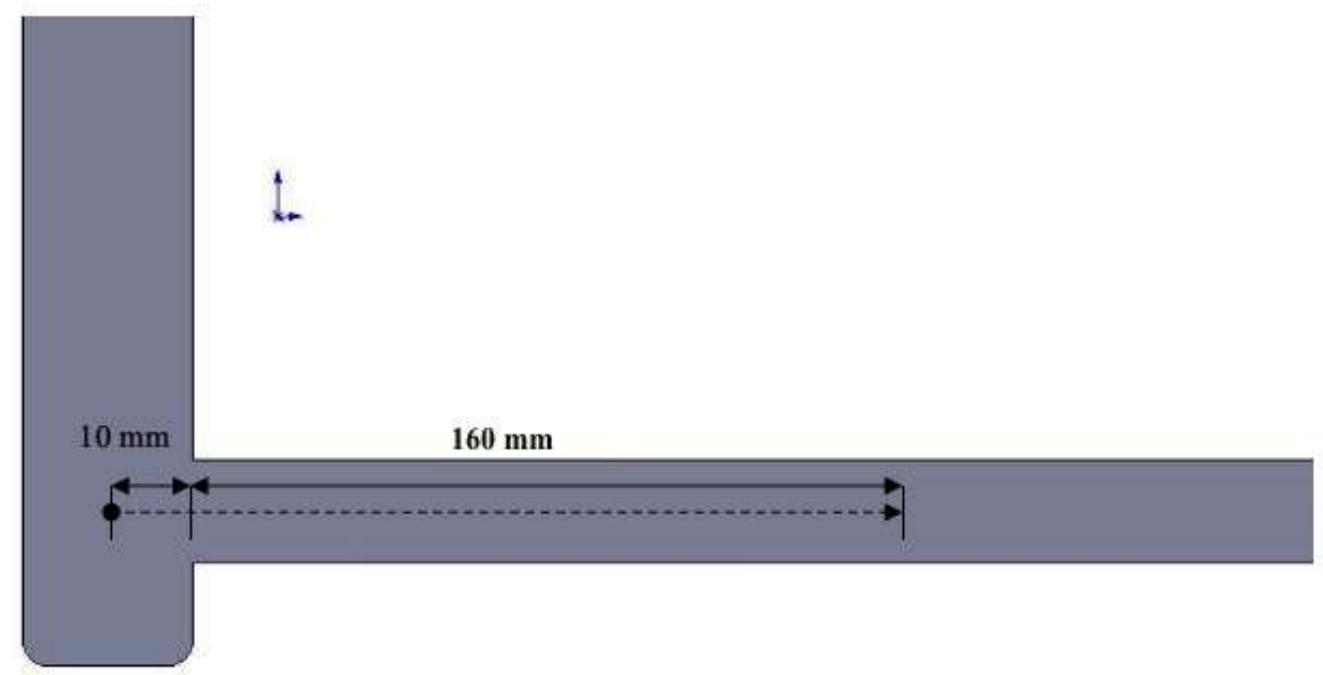

Figure 3-10. Length of linescans across horizontal bar.

The length of the scans across the downsprue of the B206 alloys is shown in Figure 3-11. The scans were initiated $10 \mathrm{~mm}$ below the intersection with the bottom edge of the horizontal bar and continued along the width $(20 \mathrm{~mm})$ of the horizontal bar. Finally, the remainder of the scans was $60 \mathrm{~mm}$ long from the top of the horizontal bar.

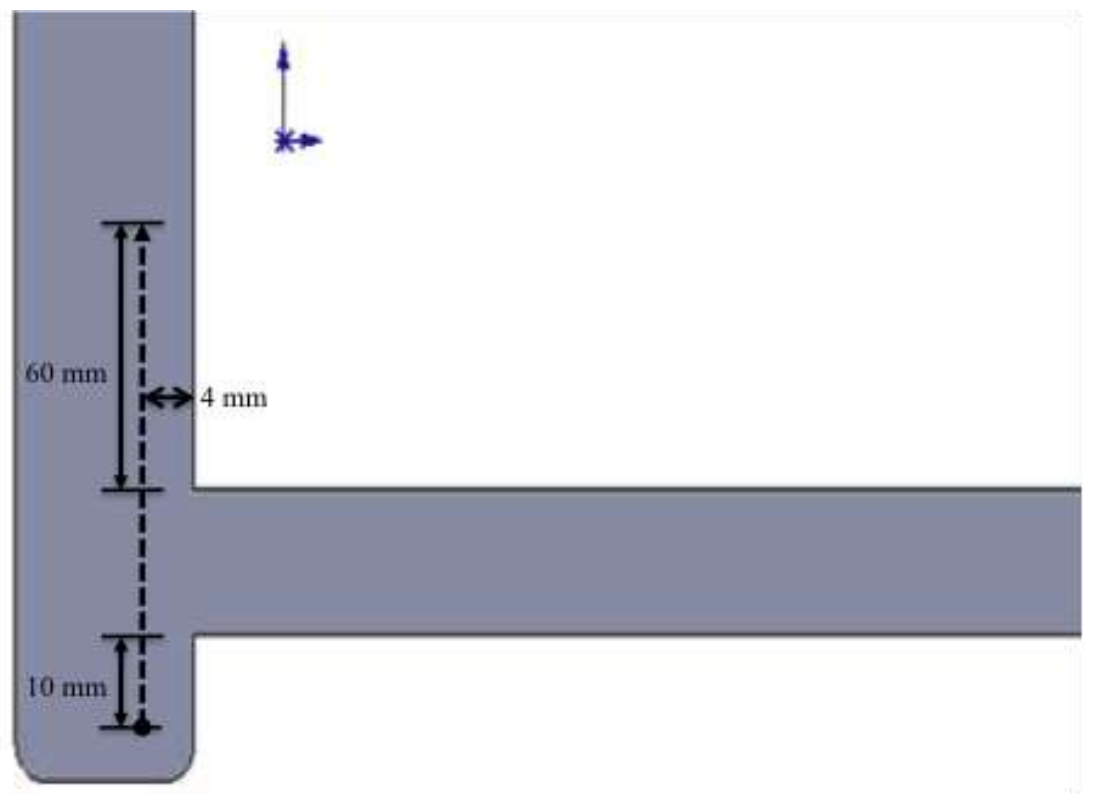

Figure 3-11. Length of linescans across downsprue. 
A summary of the linescans carried out in this research is given in Table 3-3 for each B206 casting condition. Due to the limited time allotted for neutron diffraction strain mapping experiments and the availability of the spectrometer by other users, not all linescans were completed for each direction and reflection. For instance, the centreline and downsprue scans were limited to the $x$-direction, $\varepsilon_{x}$ and (111) reflection. Previous research carried out by Bichler et al. [57] determined that strain developed in the $x$-direction was significant for the nucleation of hot tears. As a result, $\varepsilon_{x}$ was measured for all conditions and all linescans. The $y$ and $z$-direction scans were limited only to the (311) reflection. Hence, residual stress was calculated only for this reflection.

Table 3-3. Linescan summary.

\begin{tabular}{|c|c|c|c|c|c|c|c|c|c|c|c|c|}
\hline \multirow{2}{*}{ Condition } & \multicolumn{4}{|c|}{$\varepsilon_{x}$} & \multicolumn{5}{|c|}{$\varepsilon_{y}$} & \multicolumn{3}{c|}{$\varepsilon_{z}$} \\
\hline & TE & CL & BE & DS & TE & CL & BE & DS & TE & CL & BE & DS \\
\hline Unrefined & $\checkmark$ & $\checkmark$ & $\checkmark$ & $\checkmark$ & $\checkmark$ & & $\checkmark$ & & $\checkmark$ & & $\checkmark$ & \\
\hline $\mathbf{0 . 0 2}$ wt\% Ti & $\checkmark$ & $\checkmark$ & $\checkmark$ & $\checkmark$ & $\checkmark$ & & $\checkmark$ & & $\checkmark$ & & $\checkmark$ & \\
\hline $\mathbf{0 . 0 5}$ wt\% Ti & $\checkmark$ & $\checkmark$ & $\checkmark$ & $\checkmark$ & $\checkmark$ & & $\checkmark$ & & $\checkmark$ & & $\checkmark$ & \\
\hline
\end{tabular}

* TE = Top Edge; CL = Centreline; $\mathrm{BE}=$ Bottom Edge; DS = Downsprue

\subsubsection{Stress-Free Samples}

In order to calculate the residual strain in the B206 castings, it was necessary to obtain the interplanar spacing corresponding to a stress-free sample, $d_{0-h k l}$. Representative samples were therefore sectioned from the downsprue portion of the castings near the pouring cup, as shown in Figure 3-12. This portion of the casting had relatively free contraction in contrast to the section closest to the junction with the horizontal bar. Further, by machining the samples to a very small size $(3 \times 3 \times 20 \mathrm{~mm})$, it was ensured that residual stress within the samples was relieved. A minimum of three samples were extracted and analyzed for each casting condition, and the average measured interplanar spacing was used as the stress-free value. 


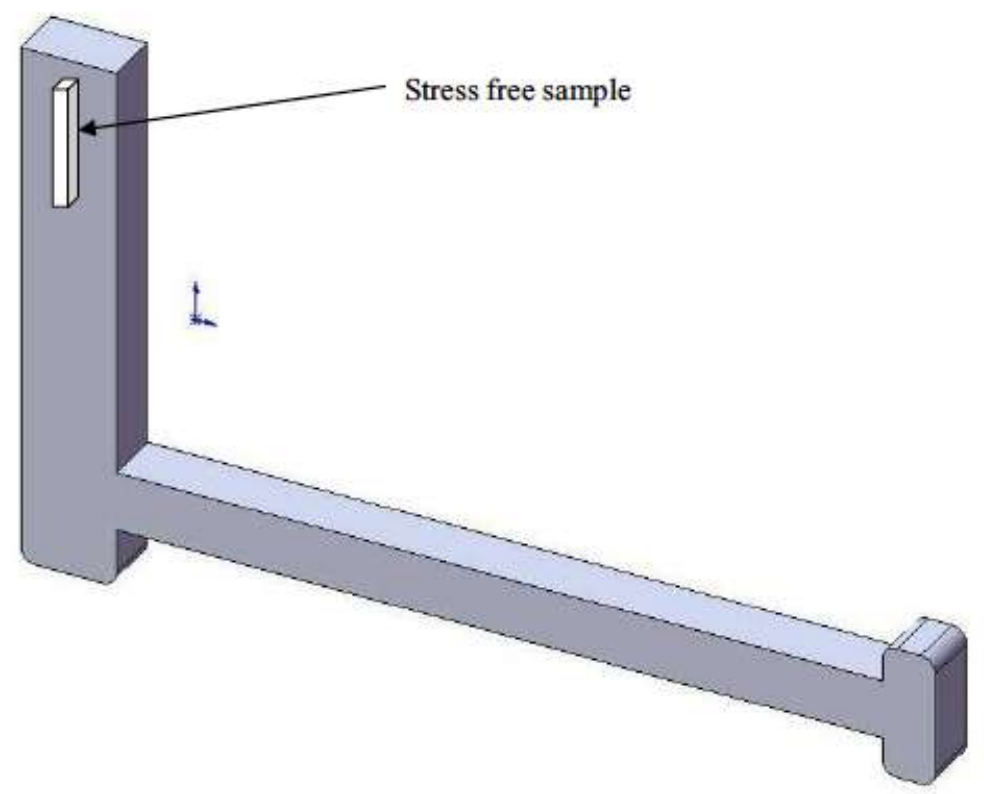

Figure 3-12. Location of extracted stress-free samples [57].

\subsection{In Situ Casting Experiments}

The in situ casting experiments were carried out at the Institute of Materials Research at Helmholtz-Zentrum Geesthacht (HZG) in Geesthacht, Germany. The purpose of these experiments was to determine the onset temperature of hot tearing in B206. This section describes the procedure undertaken for these experiments, including the design of the mold and apparatus, the casting process parameters and the method used to determine the onset temperature of hot tearing.

\subsubsection{Hot Tearing Apparatus}

The equipment developed by Zhen et al. [46] was used in this research to determine the onset temperature of hot tearing in B206. The concept of the apparatus was described in Section 2.2.3.4.1. Further details are described hereunder.

The hot tearing apparatus consisted of a CRC steel mold and a contraction force measurement system with a load cell, as shown in Figure 3-13. The load cell was connected to a data 
acquisition unit. The permanent mold was comprised of two parts: an $80 \mathrm{~mm}$ long vertical sprue and a $148 \mathrm{~mm}$ long horizontal circular rod. The diameter of the sprue was $40 \mathrm{~mm}$, while that of the rod was tapered such that it was $12.5 \mathrm{~mm}$ at the sprue and $10 \mathrm{~mm}$ at the end (by the load cell). This taper in the rod minimized the effect of friction from the mold wall (Section 2.2.3.4.1). The two parts of the mold could be disassembled into five smaller sections to facilitate the removal of the solidified casting. The mold was coated with $\mathrm{BN}$, which was removed (using a wire brush) and reapplied after each casting trial.

A threaded steel stud (6 mm diameter) was inserted into the casting cavity at one end of the rod (Figure 3-13). The stud was directly attached to the load cell via a connecting rod. The stud was designed to partially restrict contraction of the rod during solidification and initiate hot tearing. A graphite bushing (with $6 \mathrm{~mm}$ inner diameter and $10 \mathrm{~mm}$ outer diameter) was placed over the steel stud to ensure the positioning of the stud along the centreline of the horizontal rod and to prevent liquid metal from flowing out of the mold during casting. The data acquisition unit was attached to a computer and was used to record the measured force during casting. The principal function of the apparatus was to monitor the evolution of hot tearing in the casting using the measurement of contraction force induced by linear contraction of the rod. The initiation of a hot tear enabled the relief of contraction force, thereby resulting in a sudden drop on the generated force curve (Figure 3-16).

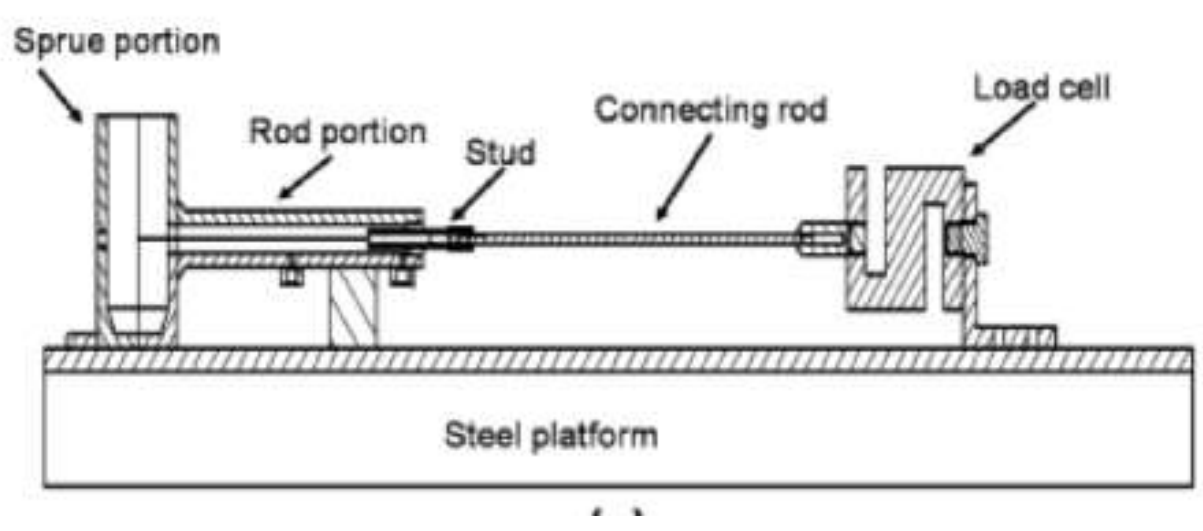

Figure 3-13. Setup of the hot tearing apparatus. 
The unrefined alloy was cast using three mold temperatures: $250{ }^{\circ} \mathrm{C}, 325{ }^{\circ} \mathrm{C}$ and $400{ }^{\circ} \mathrm{C}$. The effect of mold temperature on hot tearing in B206 was therefore investigated. In the case of the two refined alloys (i.e. $0.02 \mathrm{wt} \%$ and $0.05 \mathrm{wt} \% \mathrm{Ti}$ ), the mold temperature was kept constant at $400{ }^{\circ} \mathrm{C}$. The mold was heated to the desired temperatures using flexible heating wire wound on Kaowool ceramic blanket insulation. The wool was wrapped around the mold to enable uniform heating. Three K-type thermocouples were inserted along three locations of the mold to monitor temperature: one (T1) at the sprue-rod junction and two (T2 and T3) along the horizontal rod (Figure 3-14). At the sprue-rod junction the thermocouple was placed along the centre axis of the rod but at $\sim 5 \mathrm{~mm}$ away from the junction (i.e. inside the sprue area). This ensured that the tip of the thermocouple did not influence the initiation of hot tears. The remaining two thermocouples (T2 and T3) were inserted into the steel mold but did not come in contact with the casting rod. Therefore, they were only used to monitor mold temperature. The thermocouples were connected to the data acquisition unit. A complete setup of the apparatus is shown in Figure 3-15. Upon pouring of the metal the temperature and force data were collected and used to generate forcetemperature-time plots, which enabled the determination of the onset temperature of hot tearing.

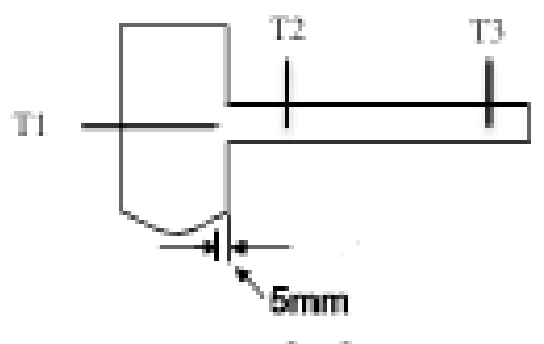

Figure 3-14. Location of thermocouples across casting.

A minimum of two repeats was carried out for each casting trial to ensure repeatability of results. The reliability of the present apparatus is well documented and reported elsewhere [46].

\subsubsection{Alloy Melting and Casting}

The B206 alloy and Al-5Ti-1B master alloy used in this part of the research were the same as those used in the ex situ casting experiments. The compositions are given in Table 3-1 and 3-2 (Section 3.1.4), respectively. Again, two levels of Ti were used: 0.02 and $0.05 \mathrm{wt} \%$. Composition 
analysis of the cast samples was carried out at Gamma Foundries and the results are presented in Appendix 1 (Table A.1-2).

The B206 alloy was melted in an electric resistance furnace. Virgin ingots were used for each casting trial. Approximately $600 \mathrm{~g}$ of the alloy was melted in a ceramic crucible. The alloy was degassed at $760{ }^{\circ} \mathrm{C}$ using $0.25 \mathrm{wt} \%$ sodium fluorosilicate. In the case of the Ti-refined alloys, the master alloy was added to the melt at $760{ }^{\circ} \mathrm{C}$, prior to adding the degasser. The melt was then held for five minutes before being mechanically stirred for one minute. Stirring of the melt ensured that the master alloy was uniformly distributed in the melt. It was not required to stir the melt when pouring the unrefined alloy. Finally, the melt was skimmed and poured at $720{ }^{\circ} \mathrm{C}$.

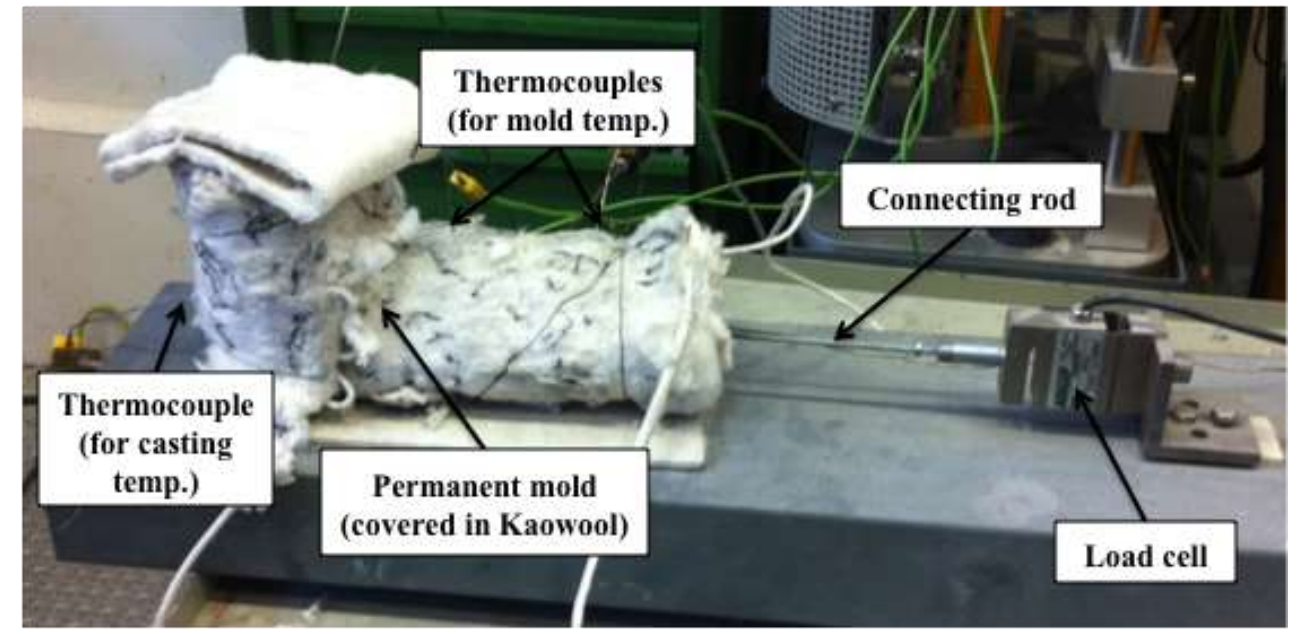

Figure 3-15. Complete setup of hot tearing apparatus.

\subsubsection{Determination of Onset Temperature of Hot Tearing}

The onset temperature of hot tearing was determined from a simultaneous plot of the force-time curve and temperature-time curve (referred to as force-temperature-time curve from hereon). An example of a force-temperature-time curve is shown in Figure 3-16. Inspection of the curves generally revealed an increased temperature in the temperature-time curves coupled with a simultaneous increase in compressive load in the force-time curve. This was likely attributed to the filling of the casting cavity by molten metal. As the molten alloy entered the casting cavity and flowed toward the load cell, the pressure against the load cell generated a compressive load. One of the limitations of the equipment was that the speed at which the melt entered the mold 
(i.e. the magnitude of compressive load) could not be controlled. Care was taken, however, to ensure the pouring speed was as similar as possible for each casting. Upon filling the casting cavity, solidification commenced and the casting began to contract with partial restriction (from the steel stud), thereby generating a relative tensile load, as indicated in the force-time curve which shows a reduction in the compressive magnitude relative to the initial drop. The load continued to increase until a relief occurred. This relief in contraction force was attributed to the formation of a hot tear. The temperature corresponding to this relief in contraction force was therefore deemed the onset temperature of hot tearing, as shown in Figure 3-16. Following this relief, the load increased towards a tensile state as the casting continued to contract. An overall tensile state likely did not result due to the initial high magnitude of compression induced by the incoming melt on the load cell.

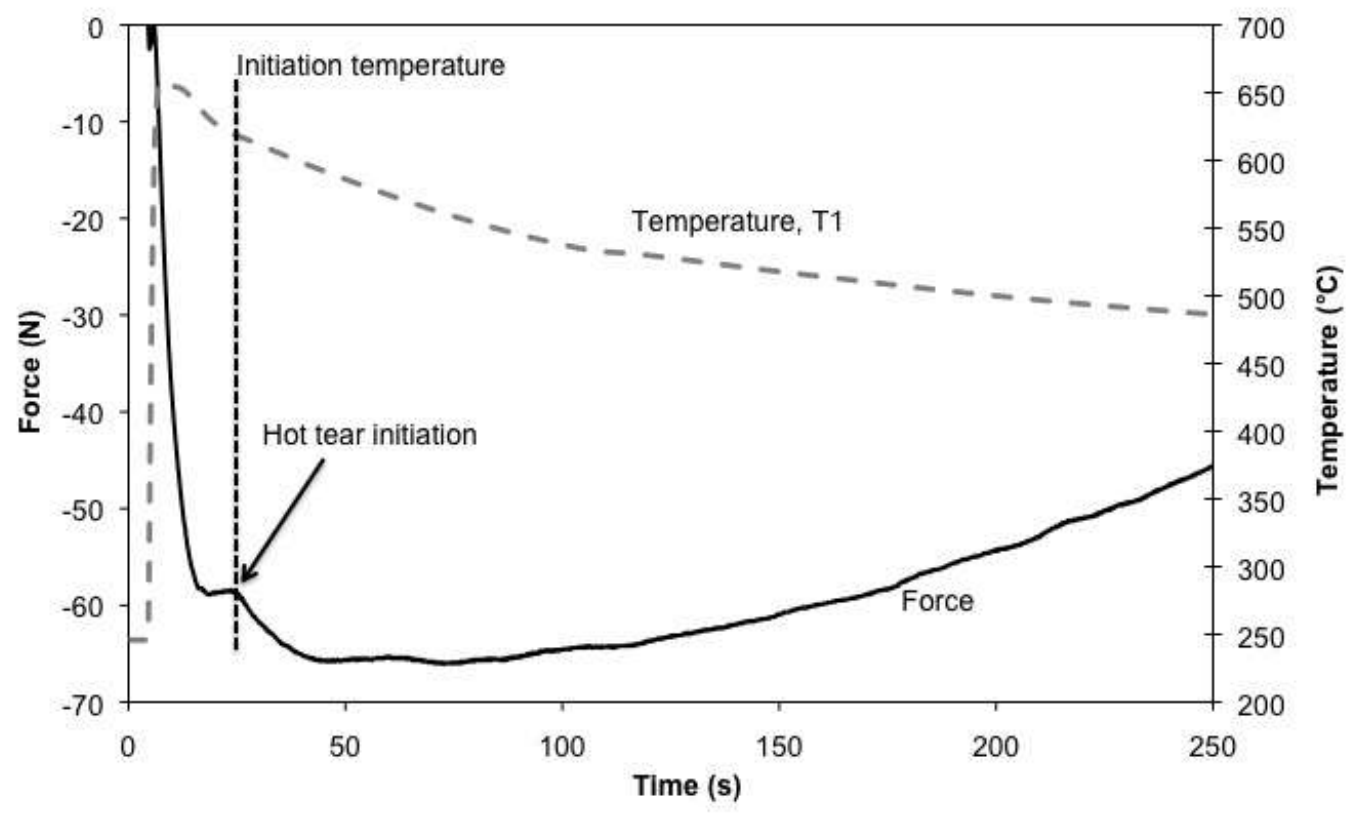

Figure 3-16. An example force-temperature-time curve for $\mathrm{B206}$.

\subsection{In Situ Neutron Diffraction Solidification Analysis}

In situ neutron diffraction solidification analysis was carried out at CNBC in Chalk River. The purpose of these experiments was to characterize the solidification kinetics of the B206 alloy and 
to determine the onset solid fraction of hot tearing. The methodology including, sample preparation, FactSage simulation and neutron diffraction experiments are presented.

\subsubsection{Preparation of Samples}

The samples used for neutron diffraction analysis were prepared at the CNPM at Ryerson University. An Al-5wt\% Cu binary alloy was used for these samples. The binary alloy was selected to minimize the effect of other alloying elements present in B206 (e.g. Si, Mn, Mg) on neutron diffraction signals. This enabled a more valid approach for using neutron diffraction as a solidification analysis tool.

The Al-5wt\% $\mathrm{Cu}$ alloy was melted in an electric resistance furnace. Virgin ingots of pure $\mathrm{Al}$ were used and additions of $\mathrm{Cu}$ were made using an $\mathrm{Al}-20 \mathrm{wt} \% \mathrm{Cu}$ master alloy. Additions of $\mathrm{Ti}$ were also made using Al-5Ti-1B master alloy (Table 3-2). A similar casting procedure as that carried out during the ex situ and in situ experiments was used. Approximately $800 \mathrm{~g}$ of the alloy was melted in a silicon carbide crucible. For each pour, the alloy was degassed at $760{ }^{\circ} \mathrm{C}$ (using $0.25 \mathrm{wt} \%$ sodium fluorosilicate), skimmed and finally poured at $720{ }^{\circ} \mathrm{C}$. The results of composition analysis carried out at Gamma Foundries are listed in Table A.1-3 (Appendix 1).

The alloy was poured into a rod-shaped steel mold preheated to $300{ }^{\circ} \mathrm{C}$, enabling a sound casting. The rod-shaped castings were then machined to the required dimensions of $40 \mathrm{~mm}$ length and $6.5 \mathrm{~mm}$ diameter, as shown in Figure 3-17. Further, a centre hole, for thermocouple placement, was drilled into the bottom face of the sample to a depth of $10 \mathrm{~mm}$ and a diameter of $2.05 \mathrm{~mm}$. These samples were then used for neutron diffraction analysis.

\subsubsection{FactSage Simulation}

FactSage is a thermochemical software package consisting of a series of thermal databases for a variety of pure materials and alloy systems. Prior to neutron diffraction analysis, FactSage simulation trials were completed for the Al-5 wt $\% \mathrm{Cu}$ alloy. The analysis was carried out over a specified temperature range for equilibrium cooling (i.e. assuming solid-state diffusion) and non- 
equilibrium cooling via Scheil (i.e. no solid-state diffusion). Such calculations provided a basis for temperature ranges for the neutron diffraction analysis.
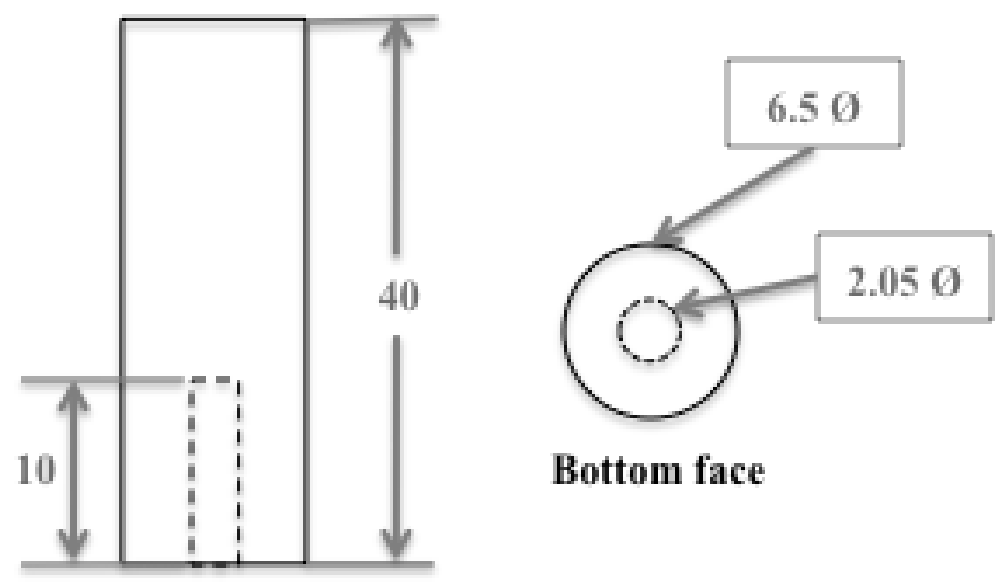

Bottom face

Figure 3-17. Dimensions of solidification sample (dimensions in $\mathbf{m m}$ ).

\subsubsection{Neutron Diffraction Analysis}

The $\mathrm{C} 2$ neutron powder diffractometer at CNBC in Chalk River was used for neutron beam analysis. A solidification cell, shown in Figure 3-18, was designed to carry out controlled melting and solidification experiments under the simultaneous exposure to neutron radiation. The $\mathrm{Al}-\mathrm{Cu}$ samples were inserted into a graphite crucible shown in Figure 3-19. The crucible inner diameter was $6.6 \mathrm{~mm}$ and the length was $64 \mathrm{~mm}$. The control thermocouple was then inserted into the sample, through a graphite sleeve (attached to a $2 \mathrm{~mm}$ wide cap), at a depth of $10 \mathrm{~mm}$. The depth of the thermocouple minimized the contribution of the thermocouple to the diffraction pattern, but ensured accurate reading of the sample temperature. A reference thermocouple was placed in the vicinity of the control thermocouple to ensure there were no significant differences in temperature. Kao-wool insulation was inserted into a slot $(11.5 \times 6.6 \mathrm{~mm})$ at the bottom of the crucible, as shown in Figure 3-19. This ensured minimal heat loss from the sample during neutron diffraction analysis. The crucible (with the sample inside) was then attached to the solidification cell (Figure 3-18b) and placed inside a vacuum furnace. The furnace infrastructure included a water inlet that enabled water to flow and cool the components of the furnace during 
the experiments. Further, argon gas was continuously supplied to the test sample to prevent it from oxidizing during the experiments.

The test sample temperature was computer controlled using a K-type sensor and PID circuit. The thermocouples used for temperature control are shown attached to the solidification cell in Figure 3-18b. The solidifying test sample was irradiated with monochromatic thermal neutrons at a wavelength of $2.37 \AA$. Diffraction patterns were collected isothermally by holding for 60 minutes at each temperature in the temperature range of $660{ }^{\circ} \mathrm{C}-440{ }^{\circ} \mathrm{C}$ at $10{ }^{\circ} \mathrm{C}$ intervals. Thus, in all, diffraction patterns were obtained for 23 temperatures. The analyzed diffraction pattern covered the angular range of 80 degrees from 37 to 117 degrees. The diffraction intensity and angle $2 \theta$ (where $\theta$ is the Bragg angle) were therefore recorded for a number of crystallographic planes with diffraction peaks within this range at each temperature.

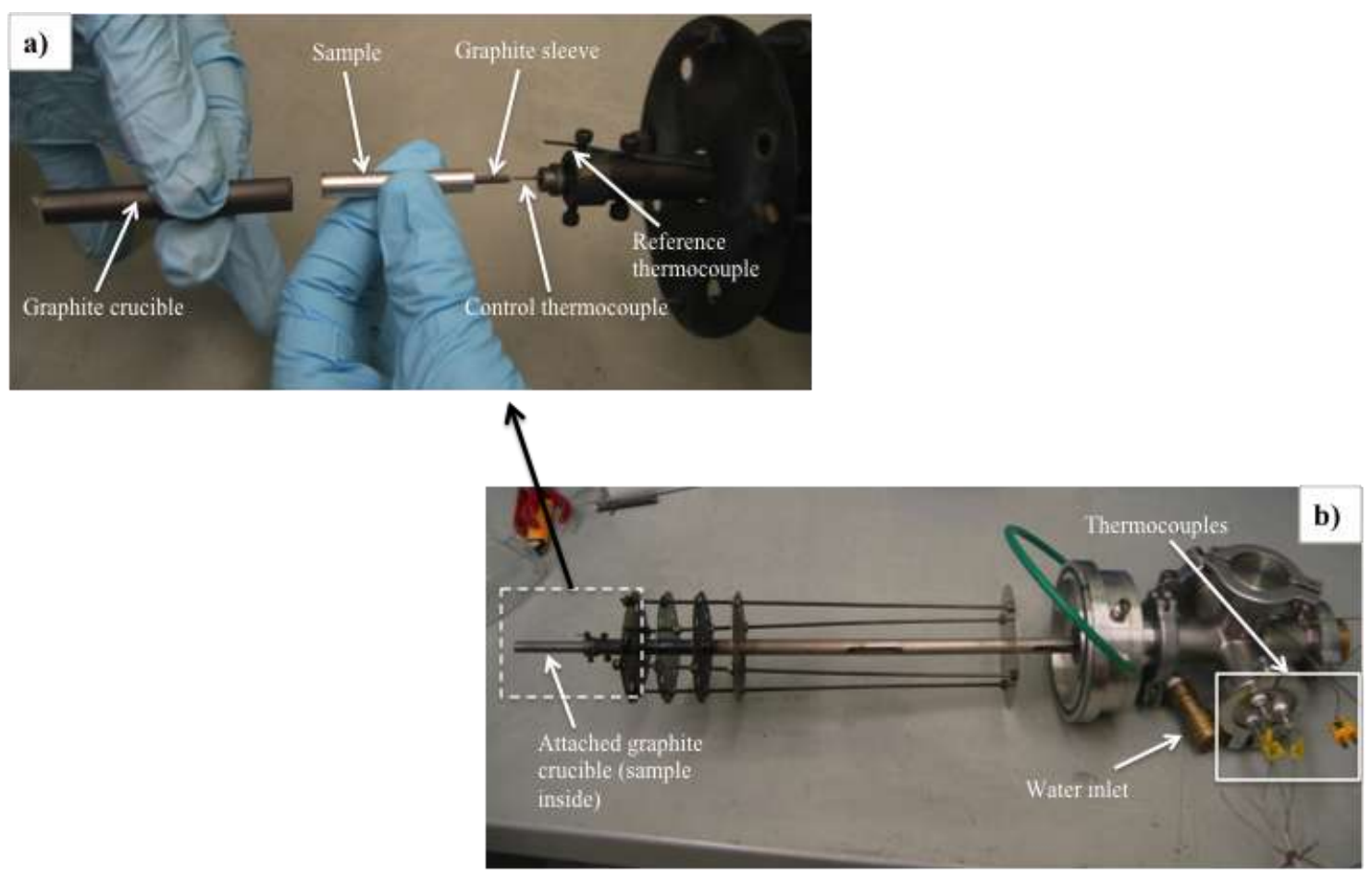

Figure 3-18. Experimental setup for neutron diffraction solidification analysis. 


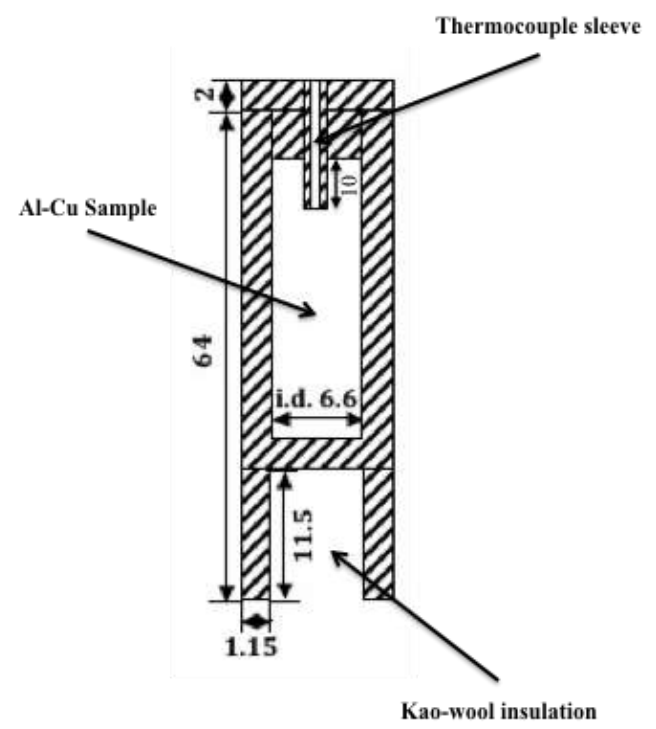

Figure 3-19. Graphite crucible (dimensions in $\mathbf{m m}$ ).

\subsection{Casting Microstructure Analysis}

Representative samples were extracted from the B206 castings for microstructural analysis. The locations from which the samples were extracted was dependent on the location of hot tears and hence, varied between castings. The sections (in Chapter 4 and 5) presenting microstructure will illustrate the exact locations of where the samples were extracted. In the case of grain size, however, the measurements were carried out along samples extracted from three locations of the horizontal bar for both the ex situ and in situ B206 castings. The three locations were in the vicinity of the thermocouples (i.e. by the sprue, in the middle and by the end) (see Figures 3-4 and 3-14).

The extracted microscopy specimens were mounted in clear Lucite using a Buehler SimpliMet 2000 automatic mounting press. The Lucite medium was allowed to cure at $150{ }^{\circ} \mathrm{C}$ for 10 minutes and was cooled with cold water for 10 minutes. The mounted specimens were then ground and polished by hand according to the procedure given in Table 3-4.

The B206 alloy was etched to view grain size using Keller's reagent. The etchant consisted of $70 \mathrm{~mL}$ of distilled water, $15 \mathrm{~mL}$ of nitric acid, $10 \mathrm{~mL}$ of hydrochloric acid and $5 \mathrm{~mL}$ of hydrofluoric acid. The solution was prepared fresh. A sample was immersed in the solution and 
the solution was agitated for 30 seconds. The sample was removed, rinsed with a mixture of warm water and ethanol and subsequently dried with a blast of hot air.

Table 3-4. Polishing procedure for microstructure analysis of samples.

\begin{tabular}{|c|c|c|}
\hline Paper Grit/ Cloth & $\begin{array}{c}\text { Wheel Speed } \\
{[\text { RPM] }}\end{array}$ & Time [min] \\
\hline 120 & $\sim 200$ & Until plane \\
\hline 320 & $\sim 200$ & 1 \\
\hline 600 & $\sim 200$ & 1 \\
\hline 1200 & $\sim 200$ & 1 \\
\hline Lecloth $+5 \mu \mathrm{m}$ alumina & $\sim 200$ & $3-5$ \\
\hline Lecloth $+3 \mu \mathrm{m}$ diamond suspension & $\sim 200$ & $2-3$ \\
\hline Lecloth $+1 \mu \mathrm{m}$ diamond suspension & $\sim 200$ & $2-3$ \\
\hline
\end{tabular}

Buehler OmniMet ${ }^{\circledR}$ image analysis software was used in conjunction with an optical microscope to characterize the microstructure and measure the grain size of the alloys. In cases when alloy grain size was too large for the optical microscope (i.e. for the unrefined alloy), grain size measurements were performed using a stereomicroscope. The linear intercept method was used for grain size measurements. A minimum of 50 measurements were taken per sample and three samples were analyzed for each casting condition.

Scanning electron microscopy (SEM) with backscattered electron detector (BSE) was carried out to examine the morphology of the alloy's secondary phases. A JEOL JSM-6380LV scanning electron microscope was used at the Department of Mechanical and Industrial Engineering at Ryerson University. Energy dispersive X-ray (EDX) analysis was also performed to estimate the chemical composition of the phases observed in the alloy. The SEM operating conditions (e.g. voltage, detector type, working distance, spot size, etc.) are given in each SEM micrograph. 


\section{Chapter 4 - Ex Situ Analysis of Hot Tearing in B206}

The results of the ex situ casting experiments carried out in this research are presented in this chapter. Each result is directly correlated to the hot tearing severity of the B206 alloy. The chapter begins with a summary of prior research that helped spur the research carried out in this dissertation. The severity of hot tearing present on the castings is illustrated in Section 4.2. Detailed microscopic analysis of the castings is presented in the following section, and the results of thermal analysis are presented in Section 4.4. Finally, the results of neutron diffraction residual strain mapping experiments are described in Section 4.5 .

\subsection{Review of Prior Research by Candidate}

The effect of grain refinement on hot tearing in B206 aluminum alloy was studied in an earlier research $[64,65]$. A series of experiments were performed whereby both mold temperature and levels of grain refiner were varied. The mold was held at various temperatures in the range of $180-380^{\circ} \mathrm{C}$ and $\mathrm{Ti}$ additions were made in four levels (i.e. 0.02, 0.05, 0.1 and $0.25 \mathrm{wt} \%$ ) in the form of Al-5Ti-1B master alloy. The results demonstrated a very high susceptibility to hot tearing for the unrefined B206 alloy. The alloy cracked at all mold temperatures, but specifically, at mold temperatures below $380{ }^{\circ} \mathrm{C}$, the tears were severe enough to completely separate the horizontal bar. As a result, a mold temperature of $380^{\circ} \mathrm{C}$ was selected in this research, to ensure that the horizontal bar of the unrefined alloy, despite cracking, remained intact. This was required in order to carry out neutron diffraction strain mapping for the unrefined alloy. Addition of Ti had a significant impact on hot tearing susceptibility. At $0.02 \mathrm{wt} \% \mathrm{Ti}$ the hot tearing severity was significantly reduced, as only a small hairline crack was observed. Finally, surface hot tears were completely eliminated at $0.05 \mathrm{wt} \%$ and beyond. As a result, the $0.05 \mathrm{wt} \% \mathrm{Ti}$ was deemed the most efficient level.

\footnotetext{
$\$$ Much of the data presented in this chapter has been published in: D’Elia, F., Ravindran, C. and Sediako, D., "Quantification of Residual Strain Associated with Reduction of Hot Tears by Grain Refinement in B206 Aluminum Alloy." Canadian Metallurgical Quarterly. Vol. 53, No. 2 (2014): 151-159; D'Elia, F., Ravindran, C. and Sediako, D., "Interplay Among Solidification, Microstructure, Residual Strain and Hot Tearing in B206 Aluminum Alloy." Materials Science and Engineering A. (accepted, August 2014).
} 
The research carried out in this dissertation builds upon prior research. Since $0.05 \mathrm{wt} \% \mathrm{Ti}$ was found to be the most efficient level with respect to eliminating hot tears, the levels of Ti used in this research did not exceed $0.05 \mathrm{wt} \%$. Further, the mold temperature was held constant at $380{ }^{\circ} \mathrm{C}$ for all casting conditions. The aim of this research was to quantitatively assess the hot tearing susceptibility of the unrefined, $0.02 \mathrm{wt} \% \mathrm{Ti}$ and $0.05 \mathrm{wt} \% \mathrm{Ti}$ conditions, as such conditions represented a casting with a full hot tear, a casting with a small hairline tear and a casting with no surface hot tear. Quantitative analysis was carried out via neutron diffraction residual strain mapping, load cell method and in situ neutron diffraction solidification analysis.

\subsection{Observation of Hot Tears on Casting Surfaces}

Upon completion of solidification, the castings were ejected from the mold and their surfaces were examined for the presence of hot tears. The casting surfaces of each alloy are shown in Figures 4-1 to 4-6. The results were in agreement with previous research [64,65]. A large hot tear was present in the middle of the horizontal bar for the unrefined alloy, as shown in Figure 4-1. The tear was severe but did not result in a separation of the bar. The junction of the downsprue and horizontal bar was also examined for hot tears, since this region was considered critical due to the $90^{\circ}$ corner. The images in Figure 4-2 confirm that hot tears were not present on the casting surface at this region.
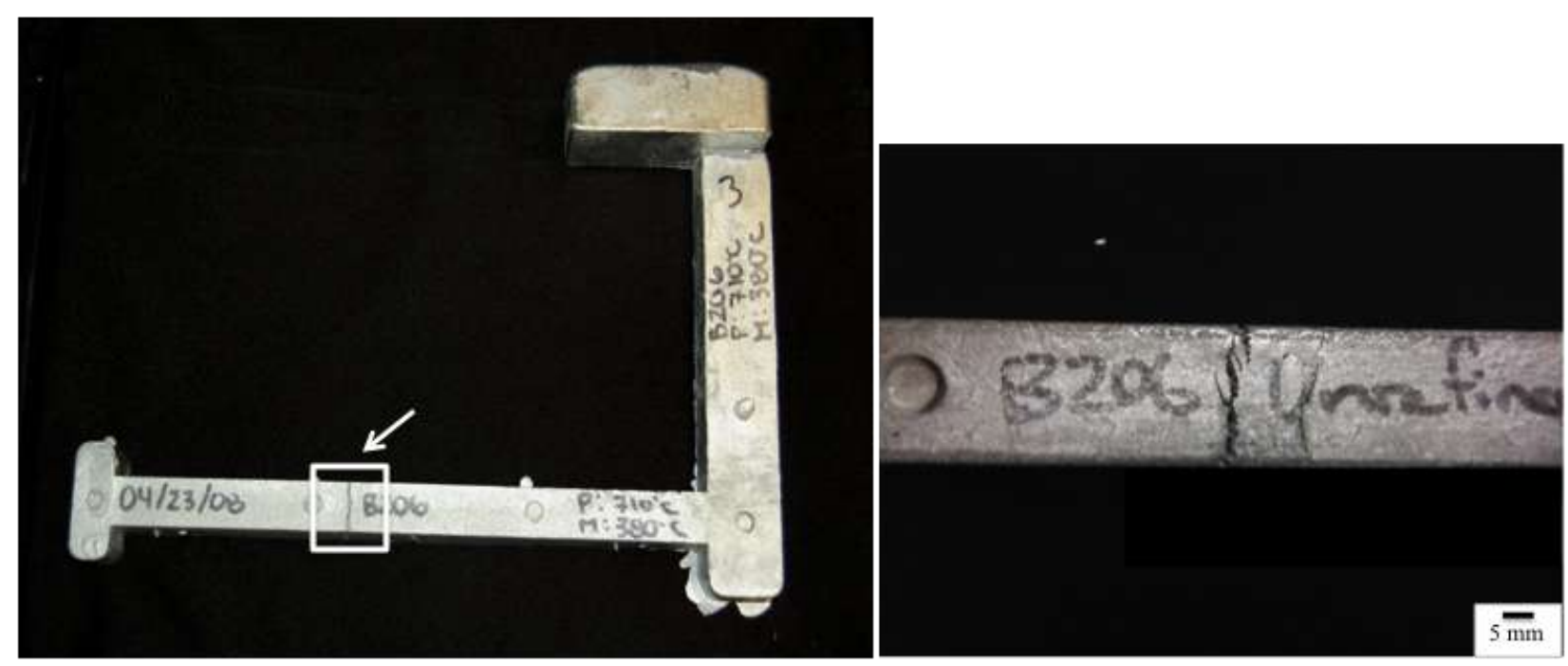

Figure 4-1. Hot tear along the middle of the horizontal bar of the unrefined B206 alloy. 
Addition of $0.02 \mathrm{wt} \%$ Ti had a significant impact on hot tearing severity in B206. The casting surface in Figure 4-3 did not reveal any hot tears along the horizontal bar. Instead, a small tear was visible at the $90^{\circ}$ sprue-bar junction, as shown in Figure 4-4. The reason for the difference in the location of the hot tear is addressed in the following sections.

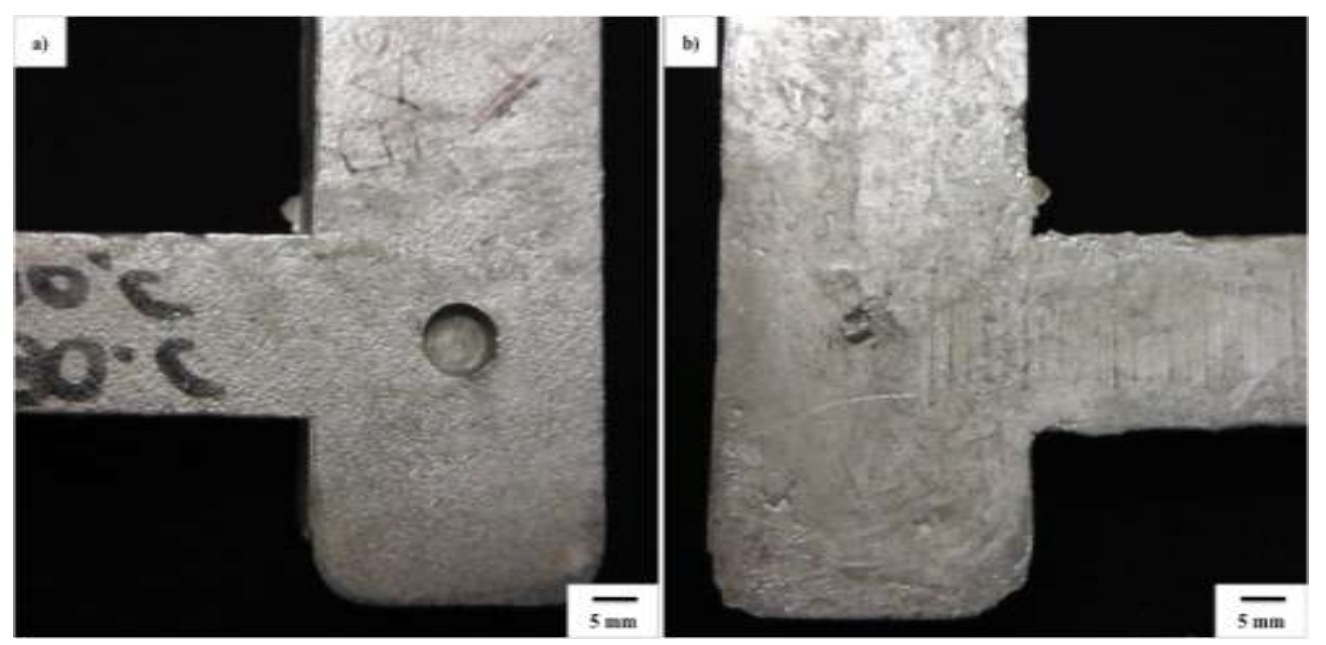

Figure 4-2. Close up of a) backside and b) front of sprue-bar junction of the unrefined B206 alloy.

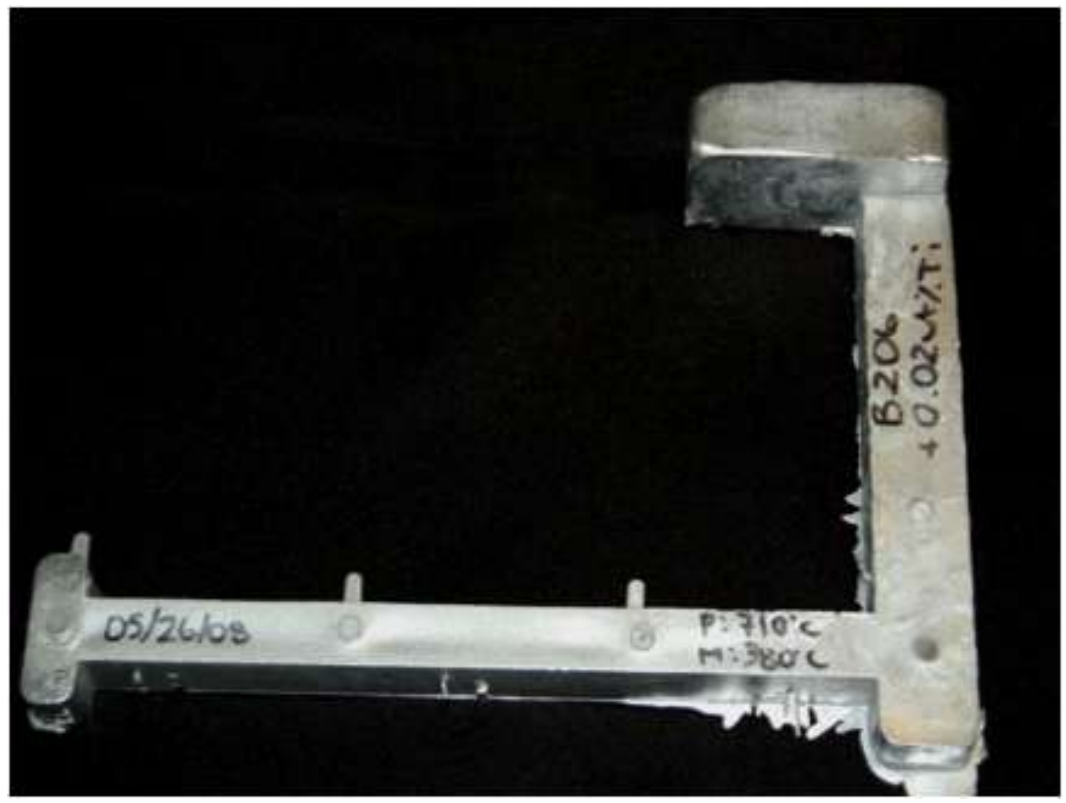

Figure 4-3. Image of casting of $0.02 \mathrm{wt} \%$ Ti B206 alloy. 
Finally, in the case of the $0.05 \mathrm{wt} \% \mathrm{Ti}$ alloy, no surface hot tears were seen along the horizontal bar, as shown in Figure 4-5. Further, examination of the critical sprue-bar junction shown in Figure 4-6 also did not reveal any hot tears for the casting condition.

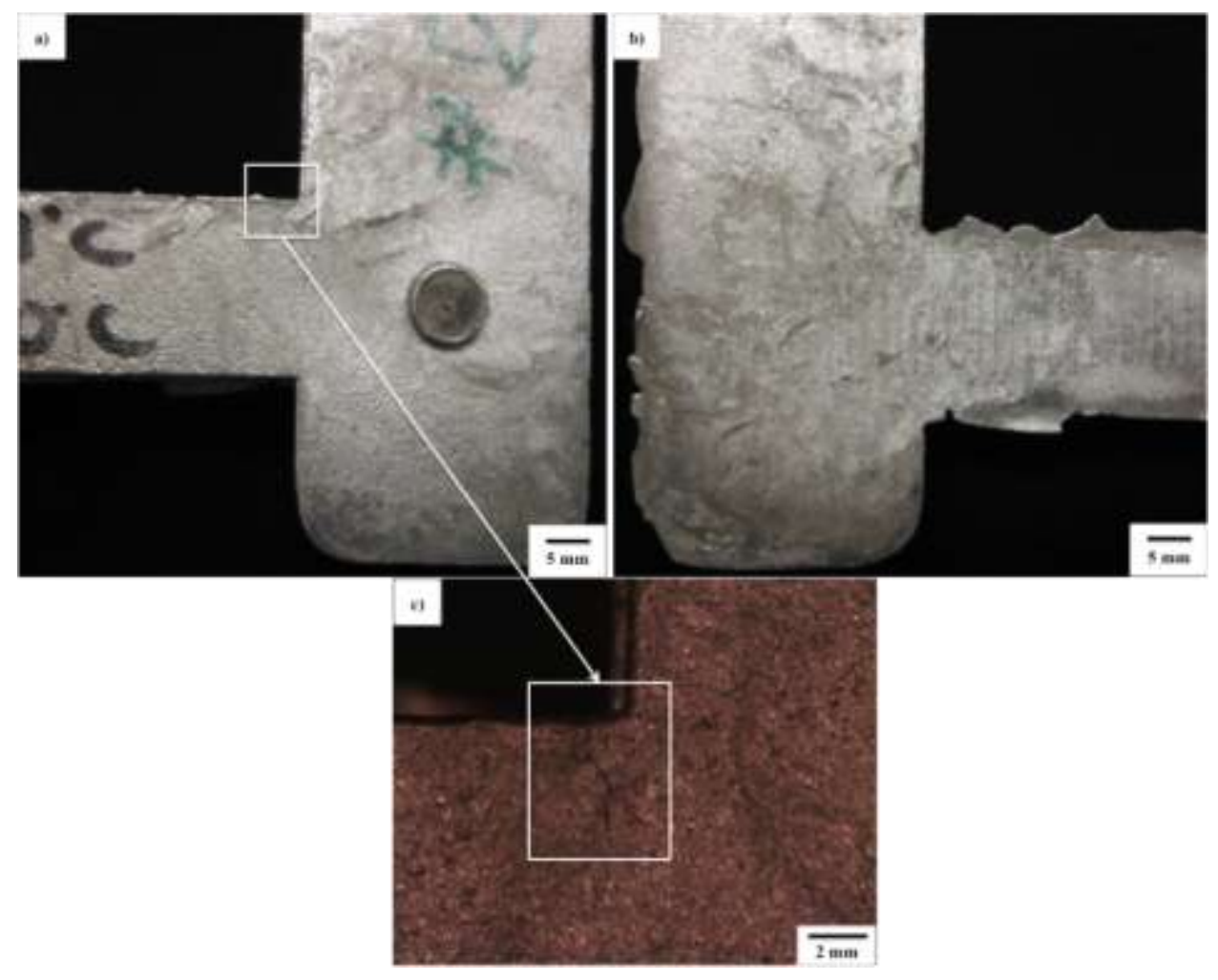

Figure 4-4. Examination of a) backside and b) front of sprue-bar junction of the 0.02 wt $\%$ Ti B206 alloy revealing c) the presence of a hot tear.

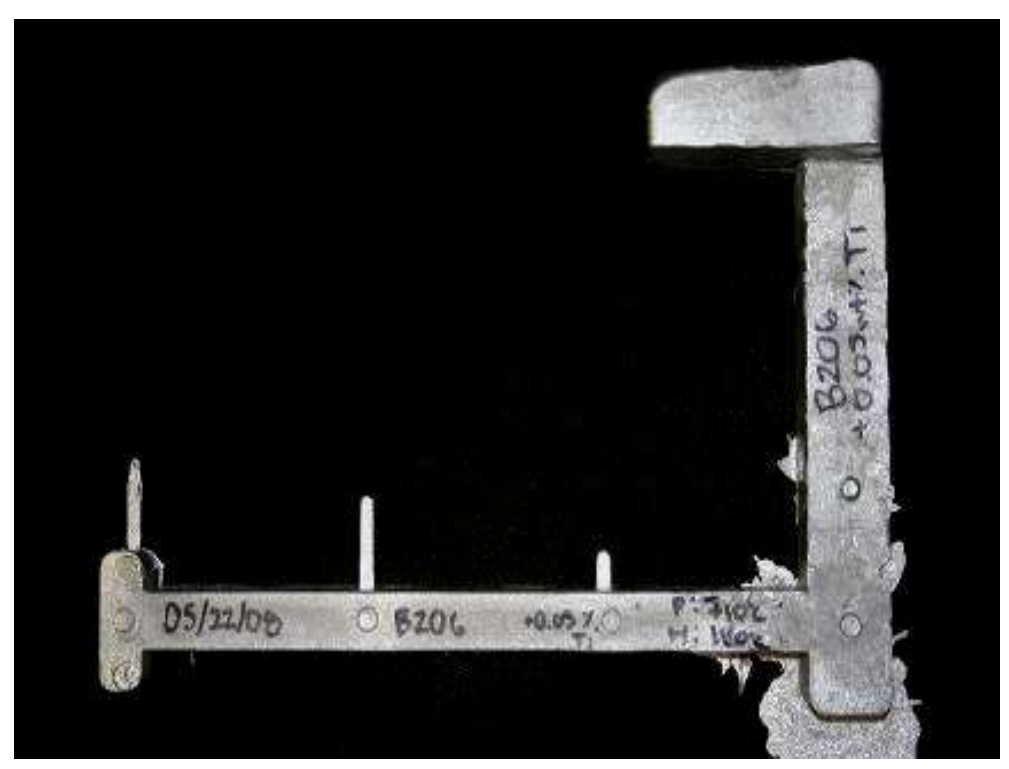

Figure 4-5. Image of casting of 0.05 wt\% Ti B206 alloy. 


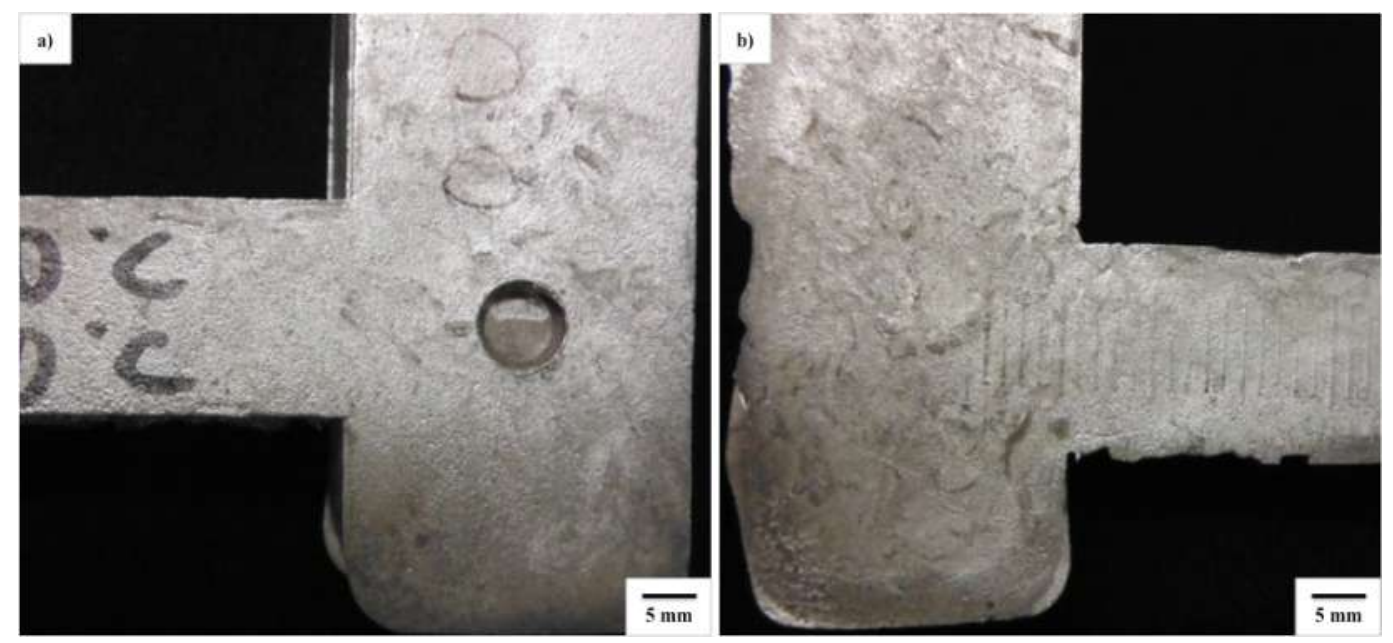

Figure 4-6. Close up of a) backside and b) front of sprue-bar junction of the $0.05 \mathrm{wt} \% \mathrm{Ti} \mathrm{B206}$ alloy.

\subsection{Casting Microstructure Analysis}

In this section, the results of optical and scanning electron microscopy for the B206 alloy are discussed. A brief description of the Al-Cu binary phase diagram and the solidification process of Al-5 wt\% Cu (e.g. B206) is given in Section 4.3.1. The grain size measurements and resulting grain morphology of the three conditions are presented in Section 4.3.2, while the effect of grain size on the distribution and morphology of second phases is analyzed in Section 4.3.3. Finally, the microstructure at the hot tear regions and the measurements of casting porosity in B206 are presented in Sections 4.3.4 and 4.3.5, respectively.

\subsubsection{Al-Cu Phase Diagram}

Phase diagrams are generally used to understand the expected solidification and subsequent development of microstructure for an alloy. For instance, the phases expected to form, the temperatures at which the phases form and the relative amounts of each phase can all be determined from the phase diagram [66]. The binary Al-Cu phase diagram (Al-rich side) is presented in Figure 4-7. The solid black line represents the equilibrium solidification of the binary Al-5 wt\% $\mathrm{Cu}$ alloy (e.g. B206). From the phase diagram, it can be seen that the liquidus temperature of this alloy is $\sim 650{ }^{\circ} \mathrm{C}$. At this temperature, primary $(\alpha) \mathrm{Al}$ crystals begin to nucleate within the liquid and subsequently develop into $\alpha$-Al grains as solidification progresses. 
The number of nuclei in the melt dictates the number of grains and is therefore the controlling factor of grain size. As the temperature decreases, these $\alpha$-Al grains containing dissolved $\mathrm{Cu}$ in solid solution, continue to grow. This process continues until the solidus temperature is reached at $\sim 560{ }^{\circ} \mathrm{C}$. At this stage, the alloy is completely solid and consists solely of $\alpha$-Al solid solution. Further decreases in temperature result in the precipitation of $\theta-\mathrm{Al}_{2} \mathrm{Cu}$ at $\sim 530{ }^{\circ} \mathrm{C}$ through solidstate diffusion. As a result, the expected microstructure of B206 (at room temperature) consists of $\alpha$ - $\mathrm{Al}$ grains surrounded by intermetallic $\mathrm{Al}_{2} \mathrm{Cu}$ at grain boundary regions.

\subsubsection{Grain Size and Morphology}

The results of grain size measurements are shown in Table 4-1. The tabulated values are the average measurements for three locations along the horizontal bar of each condition. Addition of Ti-B master alloy was found to significantly reduce the grain size of B206. For instance, $0.02 \mathrm{wt} \% \mathrm{Ti}$ addition resulted in almost a ten-fold reduction in average grain size from $1062 \pm 399 \mu \mathrm{m}$ to $105 \pm 30 \mu \mathrm{m}$. Additional Ti reduced the grain size to an average of $79 \pm 5 \mu \mathrm{m}$. These results are in agreement with the majority of literature presented in Section 2.2.2, suggesting a significant decrease in hot tearing severity with a reduction in grain size.

Table 4-1. Grain size measurements for B206 with various levels of Ti.

\begin{tabular}{|c|c|}
\hline Condition & $\begin{array}{c}\text { Avg. Grain Size } \\
(\boldsymbol{\mu m})\end{array}$ \\
\hline Unrefined & $1062+/-399$ \\
\hline $0.02 \mathrm{wt} \% \mathrm{Ti}$ & $105+/-30$ \\
\hline $0.05 \mathrm{wt} \% \mathrm{Ti}$ & $79+/-5$ \\
\hline
\end{tabular}

The micrographs in Figures 4-8 to 4-10 illustrate the grain morphology of the B206 alloys. The unrefined B206 alloy was characterized by a very coarse and dendritic grain structure (Figure 4-8). This grain morphology supports the results of hot tearing severity observed in Figure 4-1 (Section 4.2) for the unrefined alloy, suggesting that the dendrites were not able to move relative to one another under loading during solidification, and therefore fracture initiated 
readily in the material. In the case of the $0.02 \mathrm{wt} \% \mathrm{Ti}$ alloy, the grain morphology became more of an equiaxed dendrite structure, as there was evidence of small dendrite arms (Figure 4-9). It was likely that the interlocking of these dendrite arms contributed to the formation of a hot tear for this condition as well (Figure 4-4). However, the fact that the dendrites were equiaxed reduced the severity of cracking. Finally, with addition of $0.05 \mathrm{wt} \% \mathrm{Ti}$, the grain morphology transformed to a globular structure (Figure 4-10). These grains were likely more capable of sliding relative to one another as the casting contracted during solidification, thereby improving the alloy's ductility at elevated temperatures and enhancing the hot tear resistance for this condition.

\subsubsection{General Alloy Microstructure}

Scanning electron microscopy was carried out on the three conditions to observe the effect of Ti-B grain refiner on the morphology of the $\mathrm{Al}_{2} \mathrm{Cu}$ intermetallic phase. The SEM micrographs are shown in Figures 4-11 to 4-13 (at 100x and 500x magnification) for the unrefined, $0.02 \mathrm{wt} \% \mathrm{Ti}$ and $0.05 \mathrm{wt} \% \mathrm{Ti}$ conditions, respectively. Energy dispersive X-ray analysis confirmed the $\mathrm{Al}_{2} \mathrm{Cu}$ intermetallic, as shown in Figure 4-14. Inspection of the micrographs revealed that addition of the Ti-B grain refiner had a pronounced effect on the distribution of the intermetallic phase. In the unrefined alloy, the coarse dendrites limited the intermetallic phase only to small pockets between dendrite arms. As a result, this phase was only present in isolated areas. In the refined alloys, however, the fine grain size increased grain boundary area which enhanced the number of regions available for the intermetallic phase to precipitate. In turn, this resulted in a more dispersed distribution of $\mathrm{Al}_{2} \mathrm{Cu}$ throughout the microstructure. This dispersed network of the intermetallic phase suggests that a more uniform feeding of liquid occurred at the final stages of solidification for the refined alloys. In turn, this yielded a higher resistance to hot tear formation for the refined alloys, in particular for the $0.05 \mathrm{wt} \% \mathrm{Ti}$ alloy.

Examination of the 500x magnification micrographs in Figures 4-11 to 4-13 suggest that Ti-B grain refiner also had an impact on the morphology of $\mathrm{Al}_{2} \mathrm{Cu}$. In the case of the unrefined alloy, the intermetallic morphology was partially divorced, as islands of $\alpha$ - $\mathrm{Al}$ are seen within the $\mathrm{Al}_{2} \mathrm{Cu}$ particles. In contrast, with addition of Ti-B, the morphology was transformed into a more fully 
divorced structure, whereby the $\mathrm{Al}_{2} \mathrm{Cu}$ particles and $\alpha$-Al were completely separate. Moreover, inspection of the micrographs at higher magnification also illustrated a complete surrounding of $\mathrm{Al}$ grains with $\mathrm{Al}_{2} \mathrm{Cu}$ in the $0.05 \mathrm{wt} \%$ Ti condition, as shown in Figure 4-13. In comparison, the intermetallic is not as well connected along the grain boundaries for the other two conditions (unrefined and $0.02 \mathrm{wt} \% \mathrm{Ti}$ ). This further demonstrates the uniform late-stage feeding of liquid along grain boundaries for this condition, contributing to its high resistance to hot tearing.

Energy dispersive X-ray analysis also revealed $\mathrm{Fe}$ and $\mathrm{Mn}$-bearing particles throughout the microstructure of B206, as shown in Figure 4-15. These particles were found to lie within larger $\mathrm{Al}_{2} \mathrm{Cu}$ particles, as shown in the micrograph. A closer look at the particles in Figure 4-16 illustrates their fine size $(\sim 5 \mu \mathrm{m})$ and plate-like morphology. Their fine size suggests that these particles likely did not have an influence on hot tear formation in B206. In fact, the effect of Fe additions on hot tearing in B206 was investigated recently [68] and it was found that the Mn-Fe phase was favourable to hot tearing in comparison to a separate needle-like Fe phase. The needle-like Fe phase was not seen in the microstructure of B206 for this research, likely because the amount of Fe in the alloy was low $(\sim 0.05 \mathrm{wt} \%)$.

Titanium was also detected in the refined alloys by EDX analysis, as shown in Figure 4-17. Titanium-containing nucleating particles were found present in the centre of $\alpha$-Al grains, which suggested that heterogeneous nucleation was the grain refining mechanism in B206. This was consistent with the equiaxed grain morphology observed in Figures 4-9 and 4-10 for the refined alloys. In the case of the $0.05 \mathrm{wt} \% \mathrm{Ti}$ alloy, more Ti-containing nucleating particles were present in the melt (relative to the $0.02 \mathrm{wt} \% \mathrm{Ti}$ alloy), which resulted in a greater number of grains per given volume and finally a more refined grain morphology. Further, as more grains nucleated in the $0.05 \mathrm{wt} \% \mathrm{Ti}$ alloy, the growing grains impinged on one another more rapidly during solidification, thereby limiting the time available for dendrite arm growth. As a result, a more globular grain morphology was observed in the $0.05 \mathrm{wt} \% \mathrm{Ti}$ alloy relative to the $0.02 \mathrm{wt} \% \mathrm{Ti}$ alloy. 


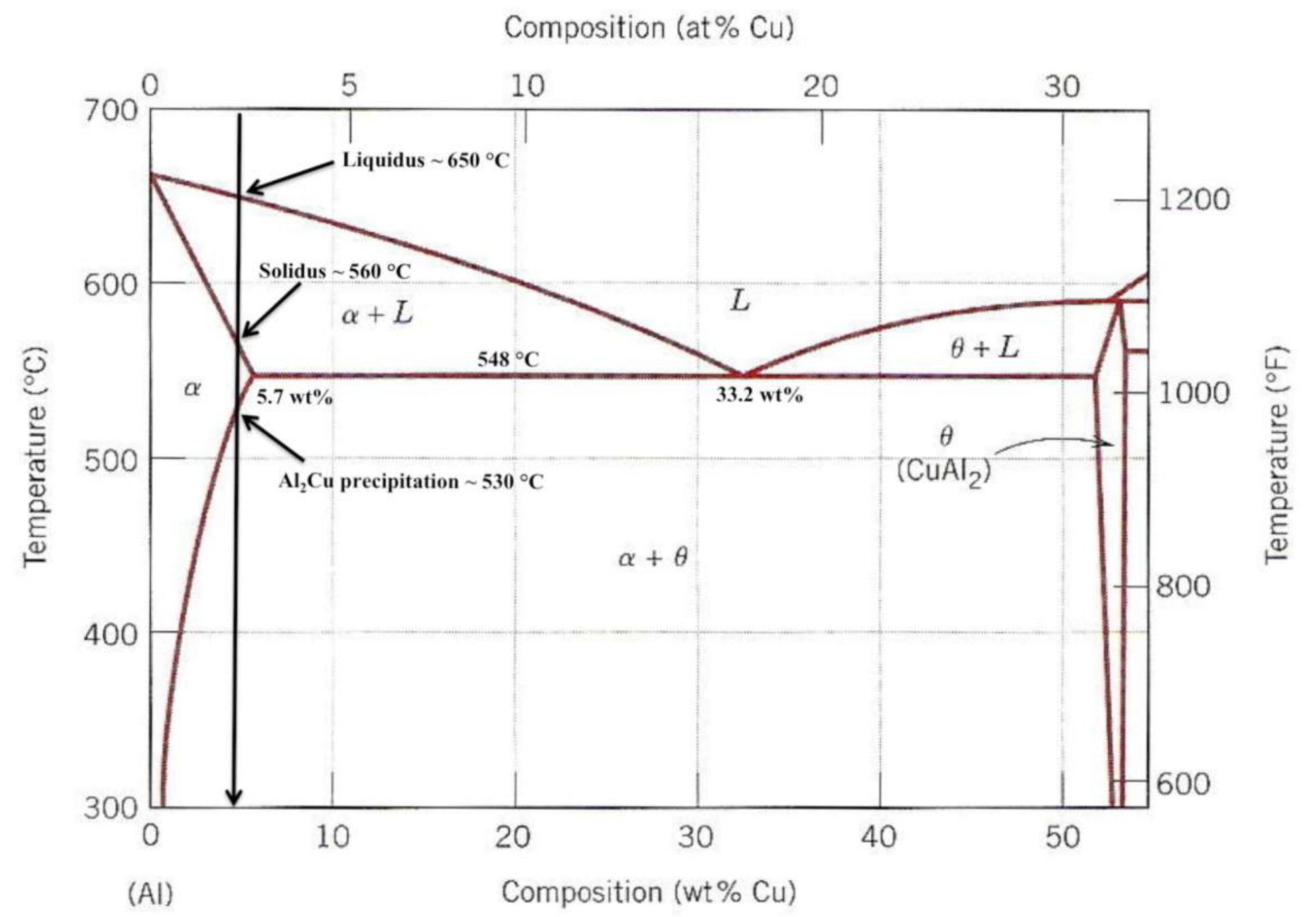

Figure 4-7. Al-Cu phase diagram (Al-rich side) [67]. 


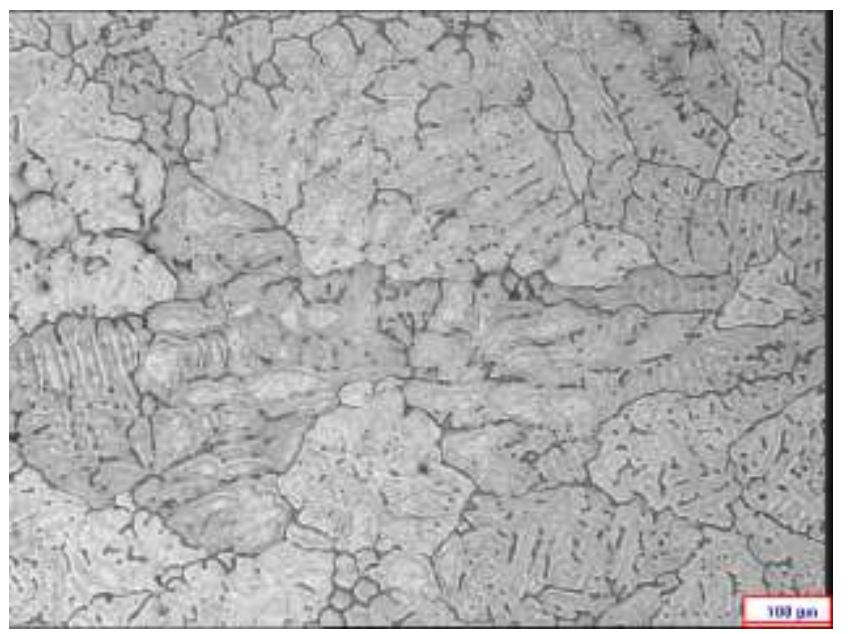

Figure 4-8. Grain morphology in unrefined B206 alloy (100x).

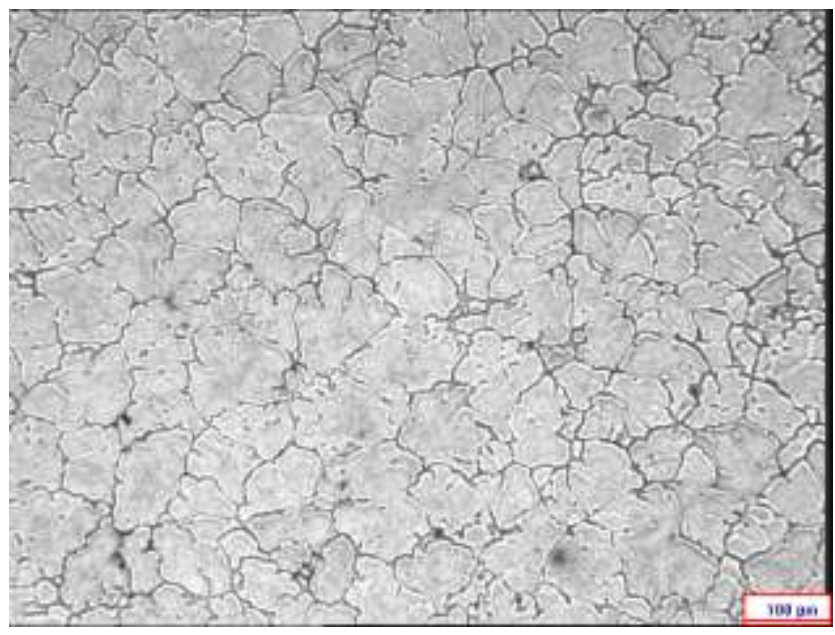

Figure 4-9. Grain morphology in 0.02 wt\% Ti alloy (100x).

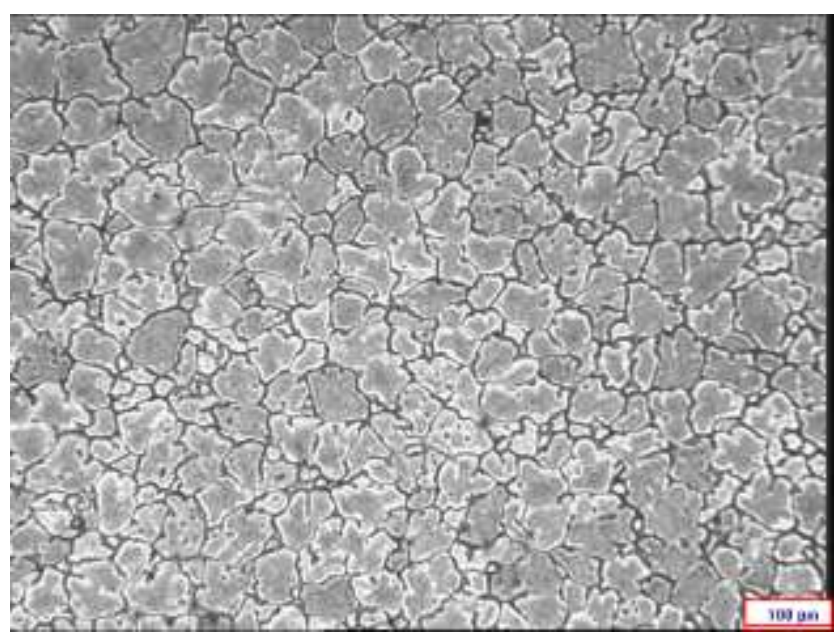

Figure 4-10. Grain morphology in 0.05 wt $\%$ Ti alloy $(100 x)$. 


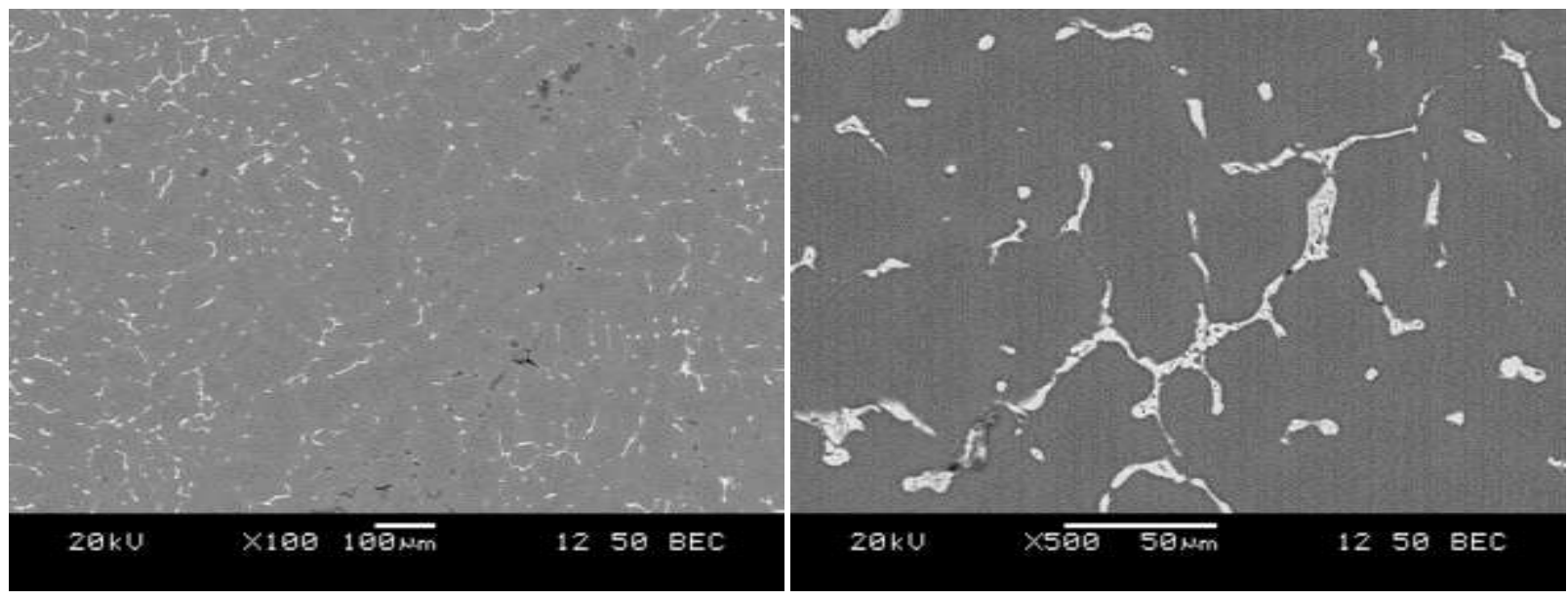

Figure 4-11. Morphology of $\mathrm{Al}_{2} \mathrm{Cu}$ intermetallic in unrefined $\mathrm{B206}$ alloy.
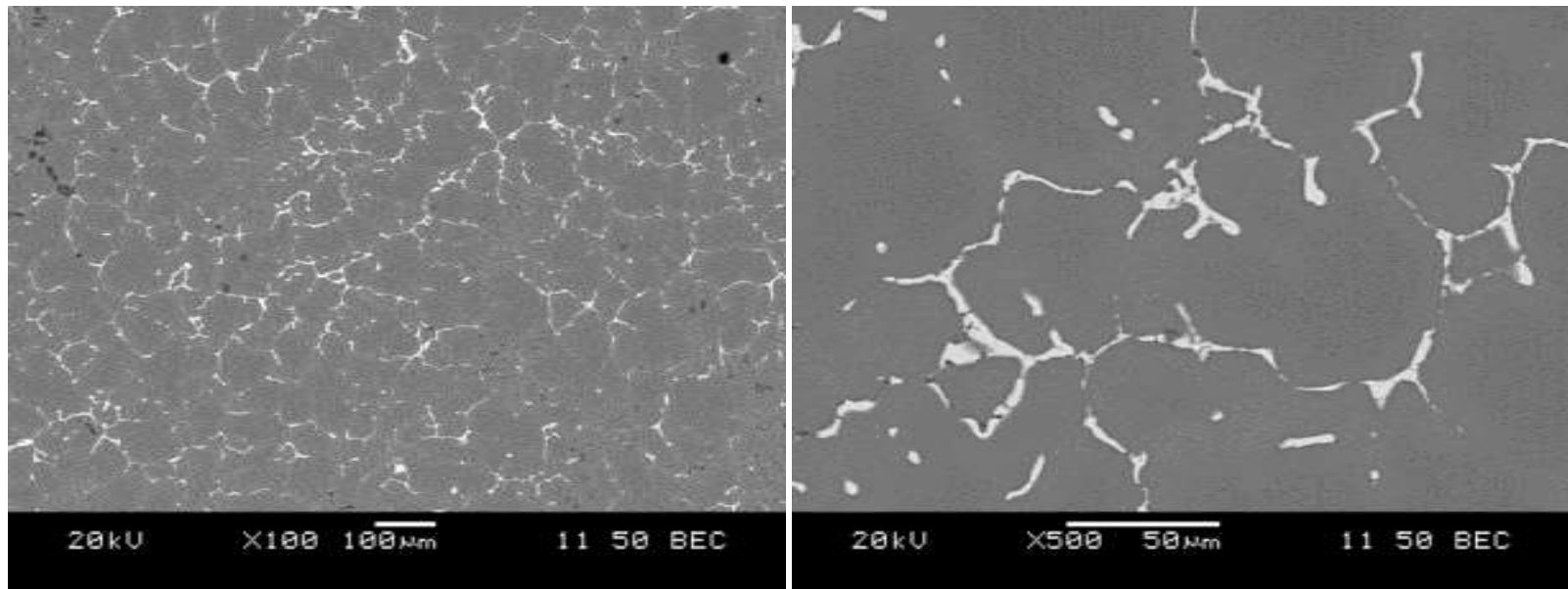

Figure 4-12. Morphology of $\mathrm{Al}_{2} \mathrm{Cu}$ intermetallic in $0.02 \mathrm{wt} \%$ Ti alloy.
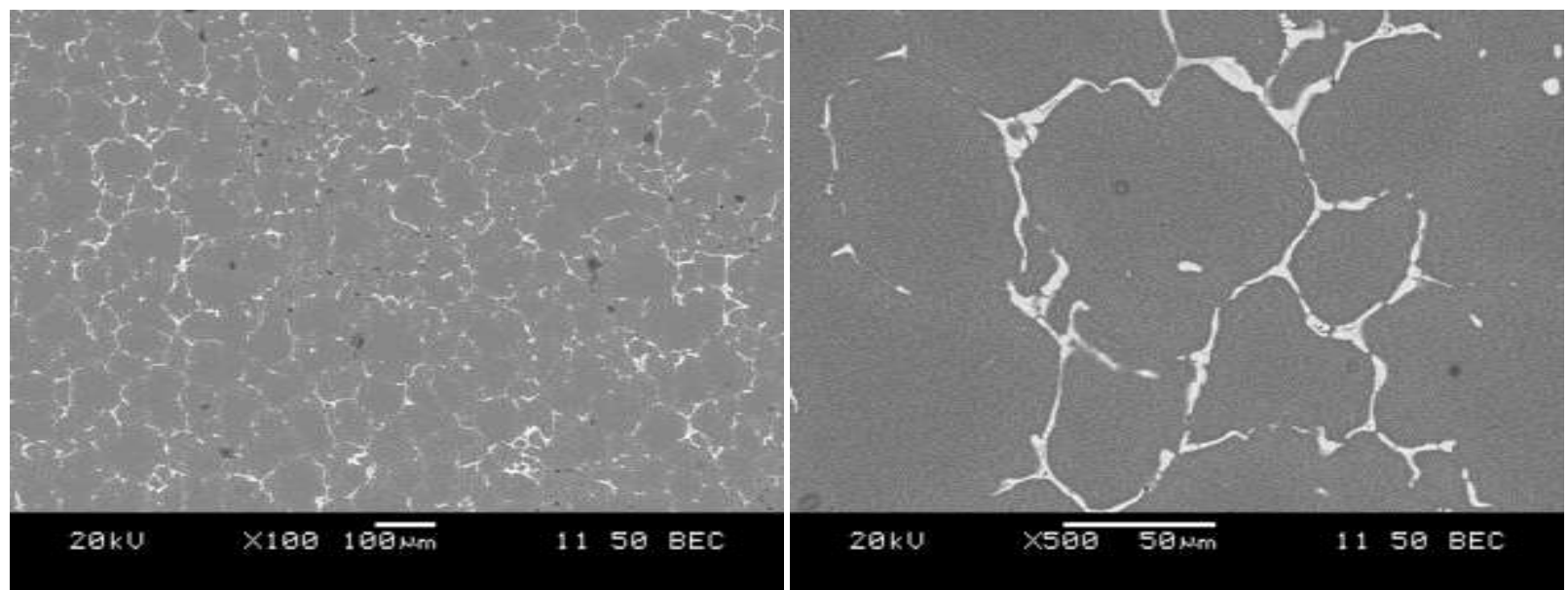

Figure 4-13. Morphology of $\mathrm{Al}_{2} \mathrm{Cu}$ intermetallic in $0.05 \mathrm{wt} \% \mathrm{Ti}$ alloy. 


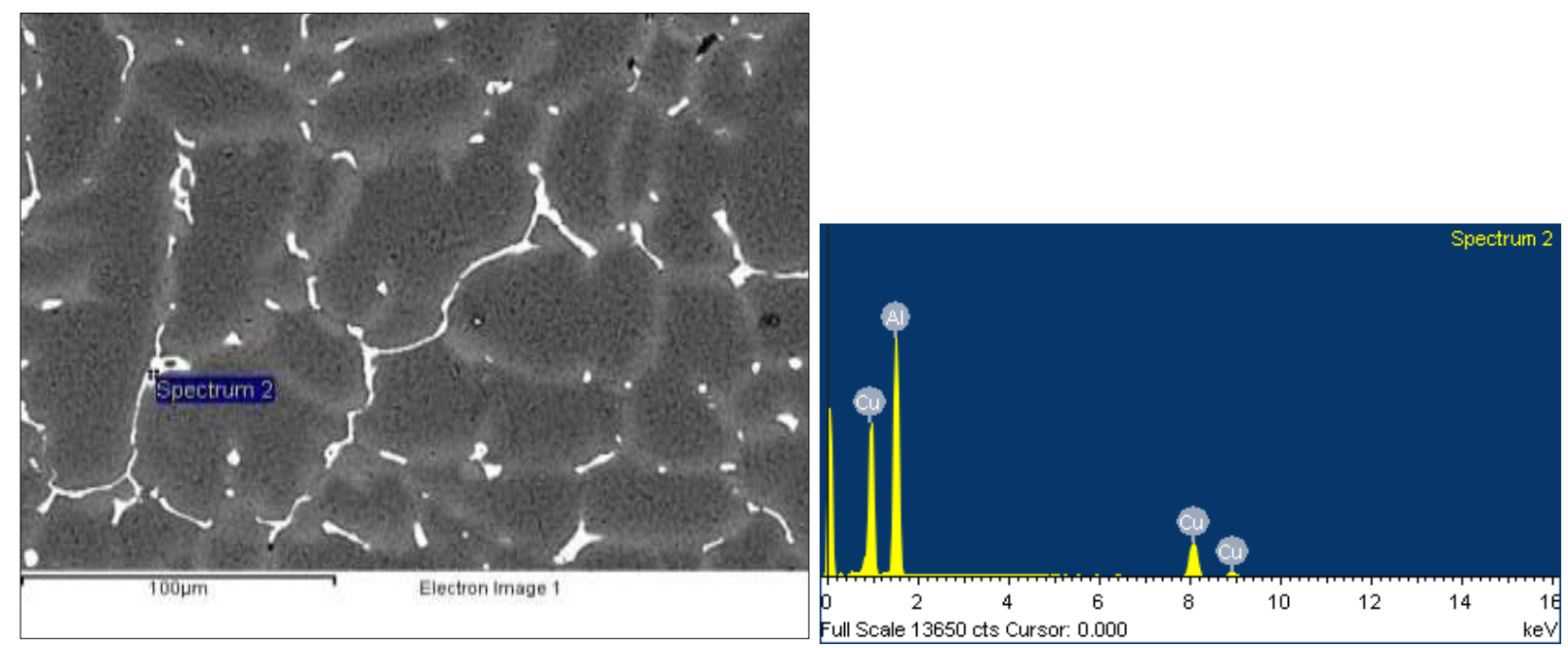

Figure 4-14. EDX analysis confirming $\mathrm{Al}_{2} \mathrm{Cu}$ intermetallic in $\mathrm{B206}$.
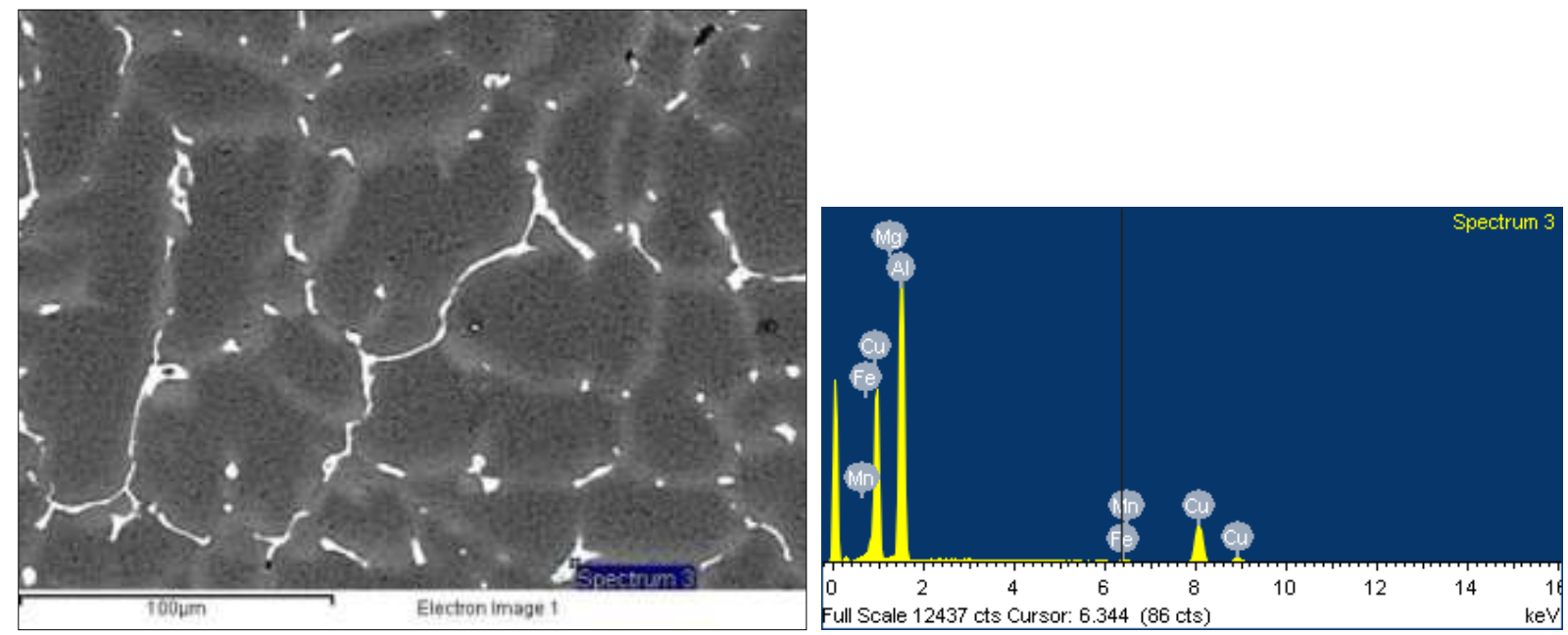

Figure 4-15. EDX analysis confirming $\mathrm{Fe}$ and Mn-bearing intermetallics in B206.

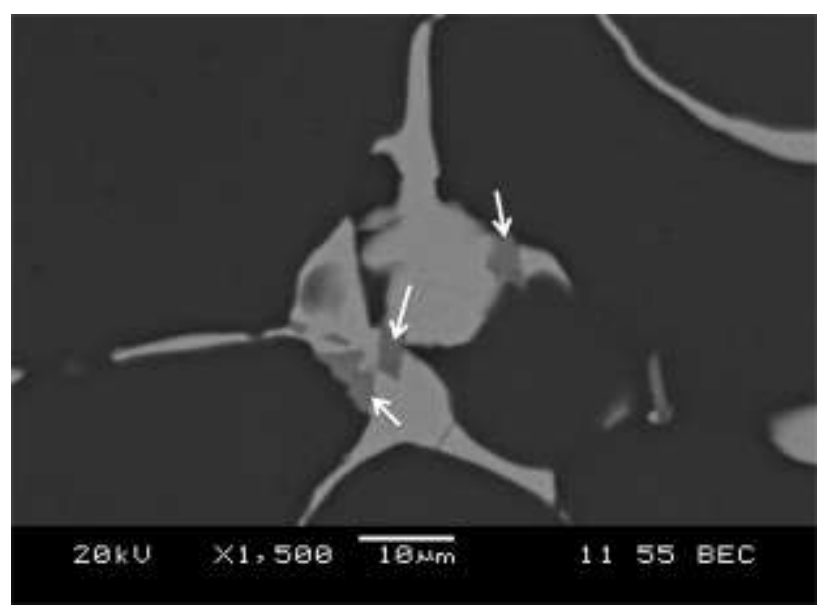

Figure 4-16. Mn and Fe-bearing particles in B206. 
There is much debate in literature as to whether $\mathrm{TiAl}_{3}$ or $\mathrm{TiB}_{2}$ particles act as nucleating particles for $\alpha$-Al grains when the Al-5Ti-1B master alloy is added to molten Al [69-75]. In this research, it was not possible to distinguish between the $\mathrm{TiAl}_{3}$ or $\mathrm{TiB}_{2}$ particle using EDX analysis due to the low atomic mass of B. Further, since this was beyond the scope of the study, no further analysis on the subject was carried out.
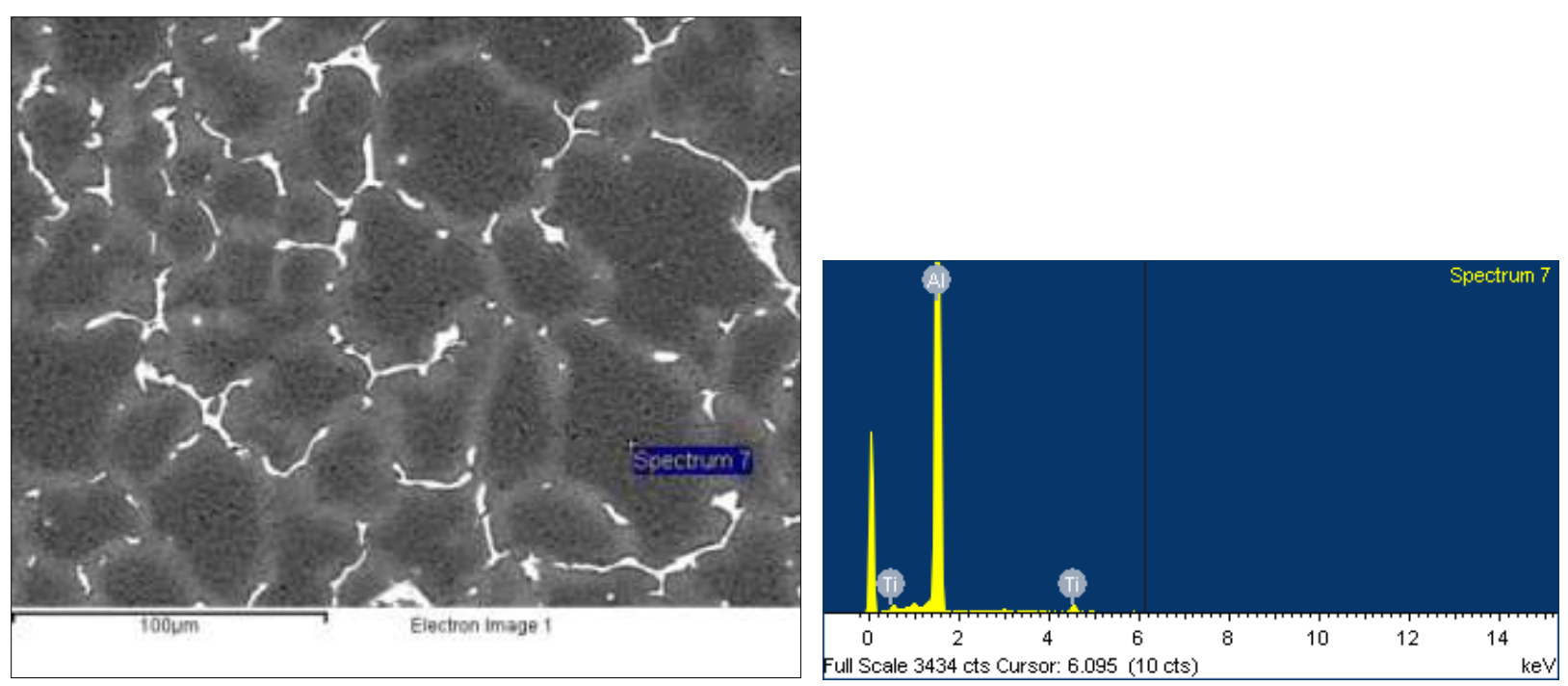

Figure 4-17. EDX analysis confirming the presence of Ti in the middle of Al grains.

\subsubsection{Analysis of Hot Tear Regions}

The images of surface hot tears on the three B206 conditions were shown and discussed in Section 4.2. Following inspection of the casting surfaces, representative samples were extracted from the castings at the $90^{\circ}$ junction between the downsprue and horizontal bar (denoted sprue-bar junction from hereon) to observe whether any internal tears were present at this location. In all, four samples were examined under a stereomicroscope: two (front and rear view) at the junction between the top edge of the horizontal bar and the downsprue and two (front and rear view) at the junction between the bottom edge of the horizontal bar and the downsprue. The location of the extracted samples is shown in Figure 4-18. Scanning electron microscopy was carried out along three (one per condition) of the 12 extracted samples and the results are presented hereunder. Further, results of SEM fractography are also presented for the unrefined alloy. The remaining stereomicrographs are given in Appendix 2. 

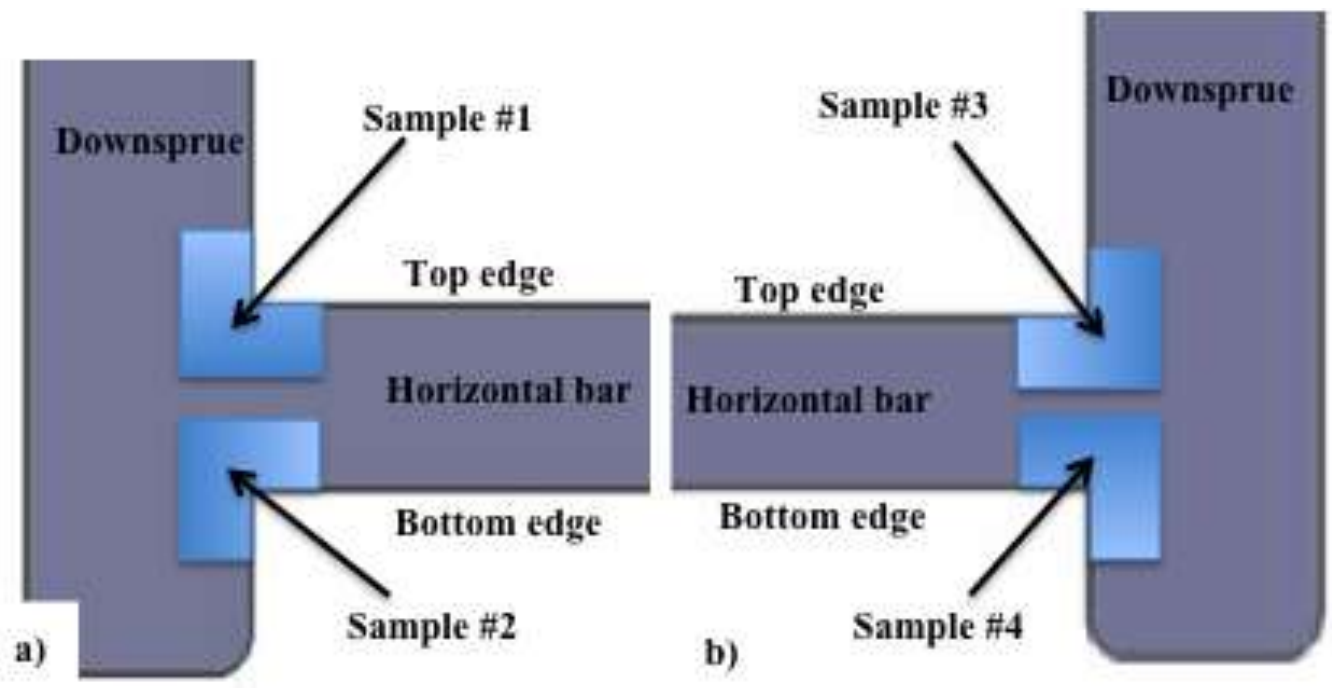

Figure 4-18. Location of extracted samples along a) front view and b) rear view of sprue-bar junction.

\subsubsection{Unrefined Alloy}

The microstructure along the sprue-bar junction for the unrefined B206 alloy is shown in Figure 4-19. The micrograph was taken from Sample \#1 in Figure 4-18. The coordinate axes corresponding to neutron diffraction analysis (see Figure 3-7) are also illustrated. Inspection of the micrograph revealed three small internal hot tears at this location. One tear was present in the downsprue portion, one in the horizontal bar and one at the junction (i.e. $90^{\circ}$ corner) $\sim 45^{\circ}$ relative to the downsprue and horizontal bar, as shown in Figure 4-19. The tears were seen to propagate into the casting along interdendritic regions. Figure 4-20 illustrates the path of the tears present in the downsprue and at the $90^{\circ}$ corner. Hot tears were expected in this region of the casting as a result of both the contraction of the downsprue and the horizontal bar. Under ideal conditions, once the molten metal fills the casting cavity and solidification begins, the downsprue would tend to contract towards the pouring cup (placed on top of the mold) (in the positive $y$-direction) while the horizontal bar would tend to contract towards the end restraint (in the positive $x$-direction). However, since both parts are attached to each other their contraction is mutually dependent on one another and in turn the likelihood of fracture becomes high at this location during casting. The measurements of residual strain presented in Section 4.5 will provide a better understanding of the strain generated along this region. 


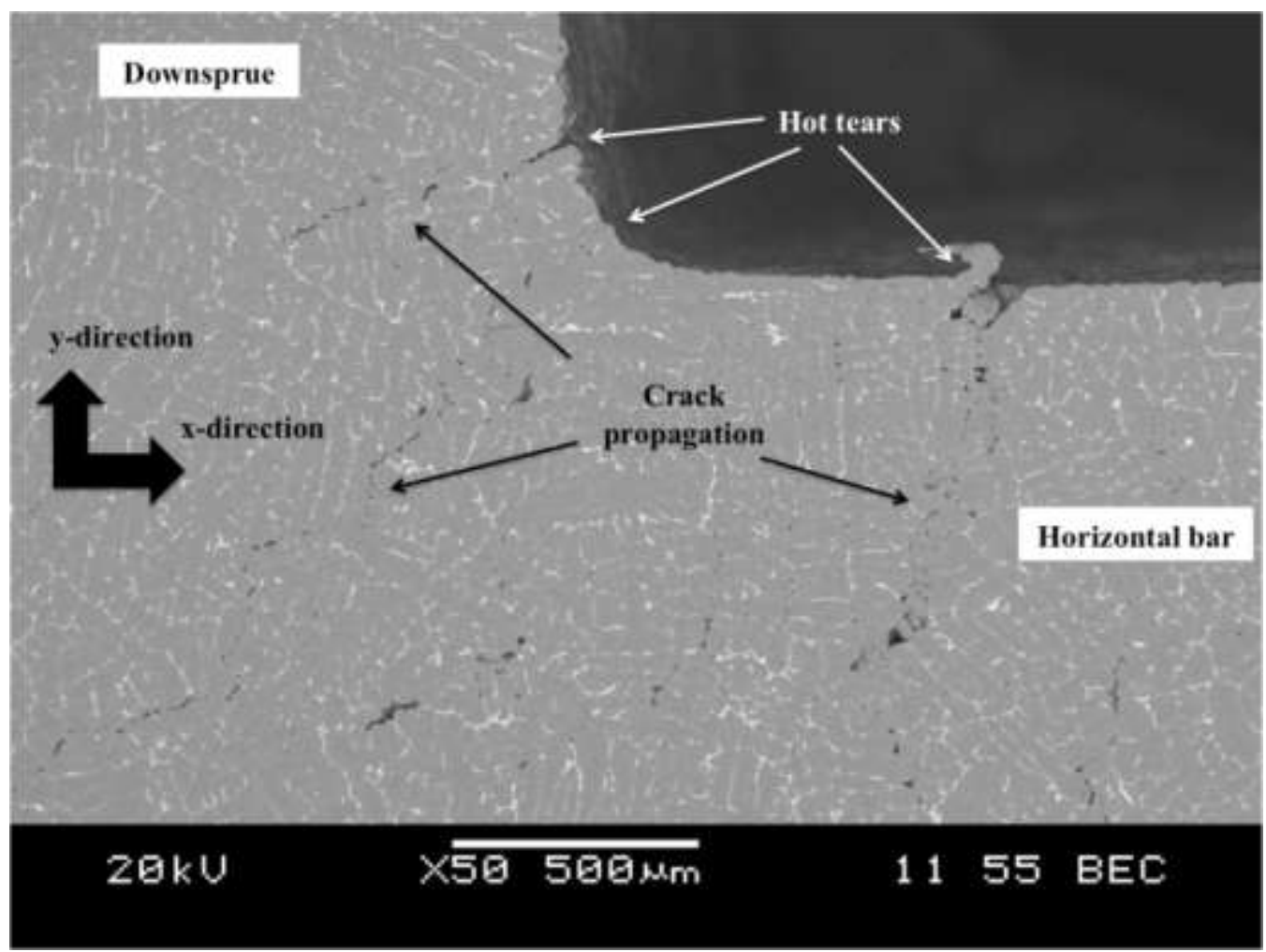

Figure 4-19. Sprue-bar junction in unrefined B206 depicting hot tears.

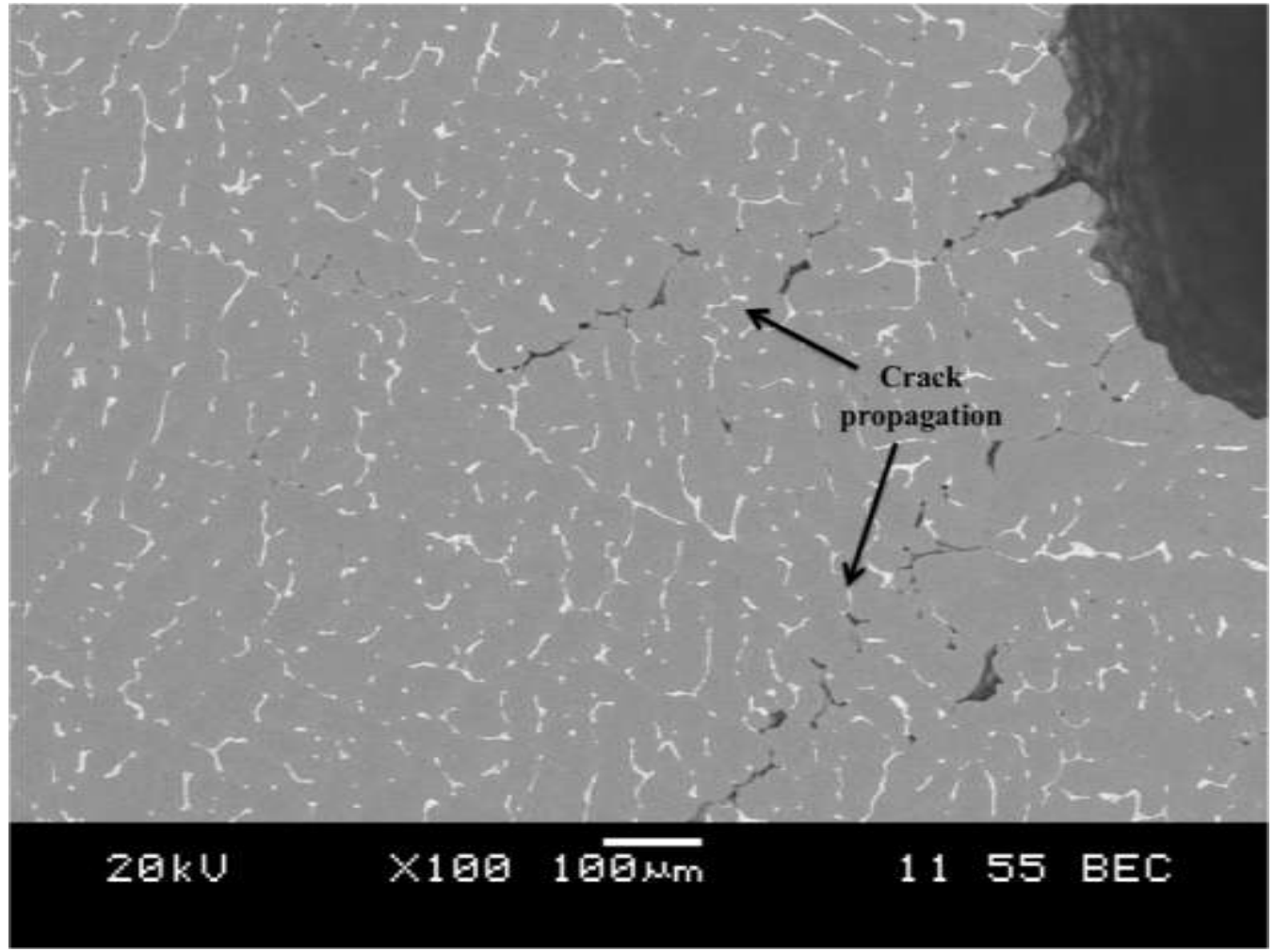

Figure 4-20. Propagation of cracks through interdendritic regions in unrefined B206. 
The fracture surface of the hot tear present at the middle of the horizontal bar for the unrefined alloy was examined using SEM. The micrograph in Figure 4-21 illustrates the dendritic morphology of the fracture surface. This confirms the interdendritic path of the hot tears. The fracture surface was examined at higher magnification and this revealed the presence of the intermetallic $\mathrm{Al}_{2} \mathrm{Cu}$ in between secondary dendrite arms and along fractured dendrites, as shown in Figure 4-22. The $\mathrm{Al}_{2} \mathrm{Cu}$ intermetallic is expected to precipitate after solidification of $\mathrm{B} 206$ is complete, as suggested by the Al-Cu phase diagram (Figure 4-7). Thus, this phase likely did not play a significant role in the formation and propagation of hot tears in B206, as it precipitated once hot tears were already present in the alloy. A further analysis on the in situ precipitation and growth of $\mathrm{Al}_{2} \mathrm{Cu}$ is presented in Chapter 5.

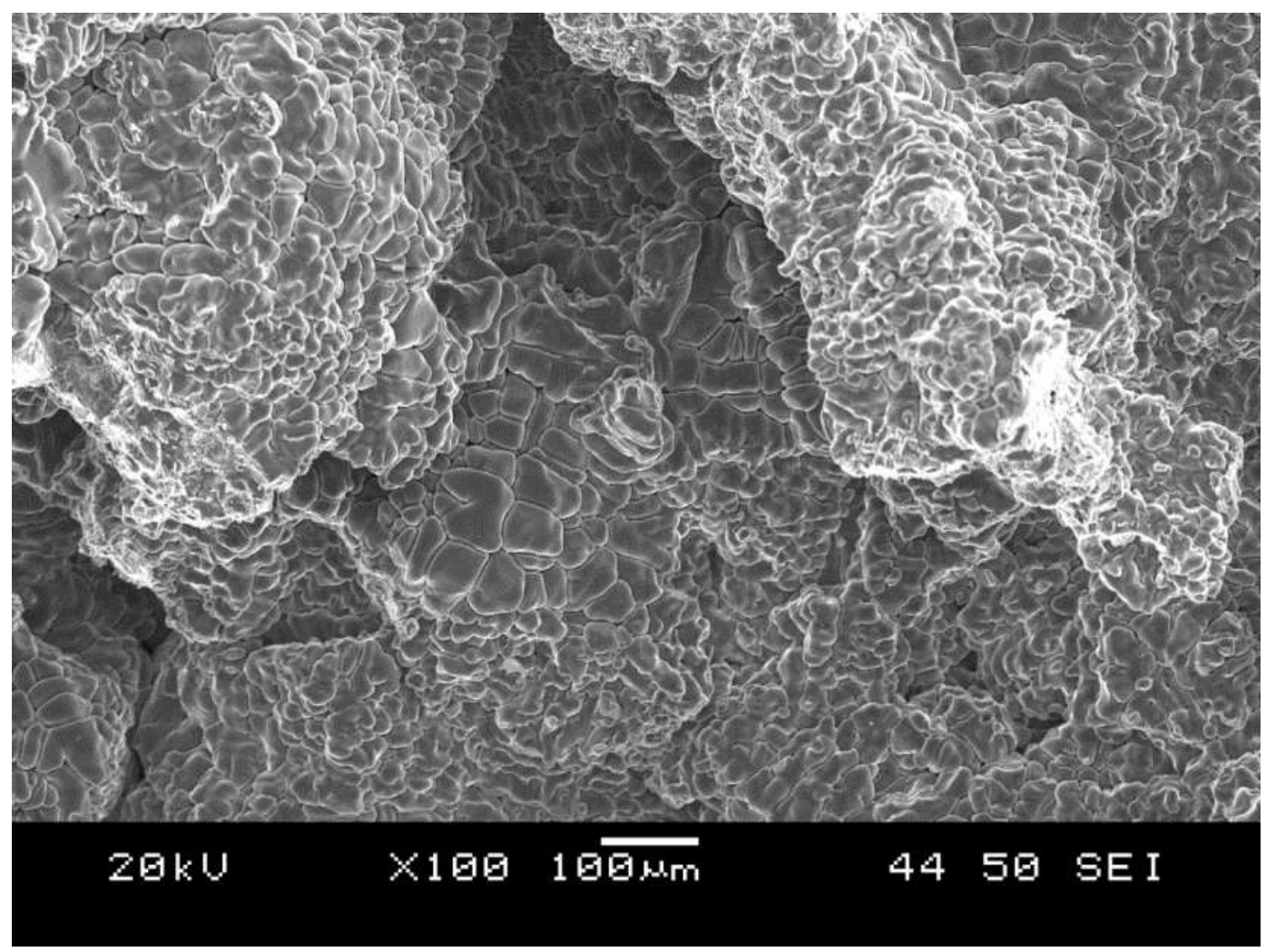

Figure 4-21. Dendritic morphology along hot tear fracture surface in unrefined B206. 


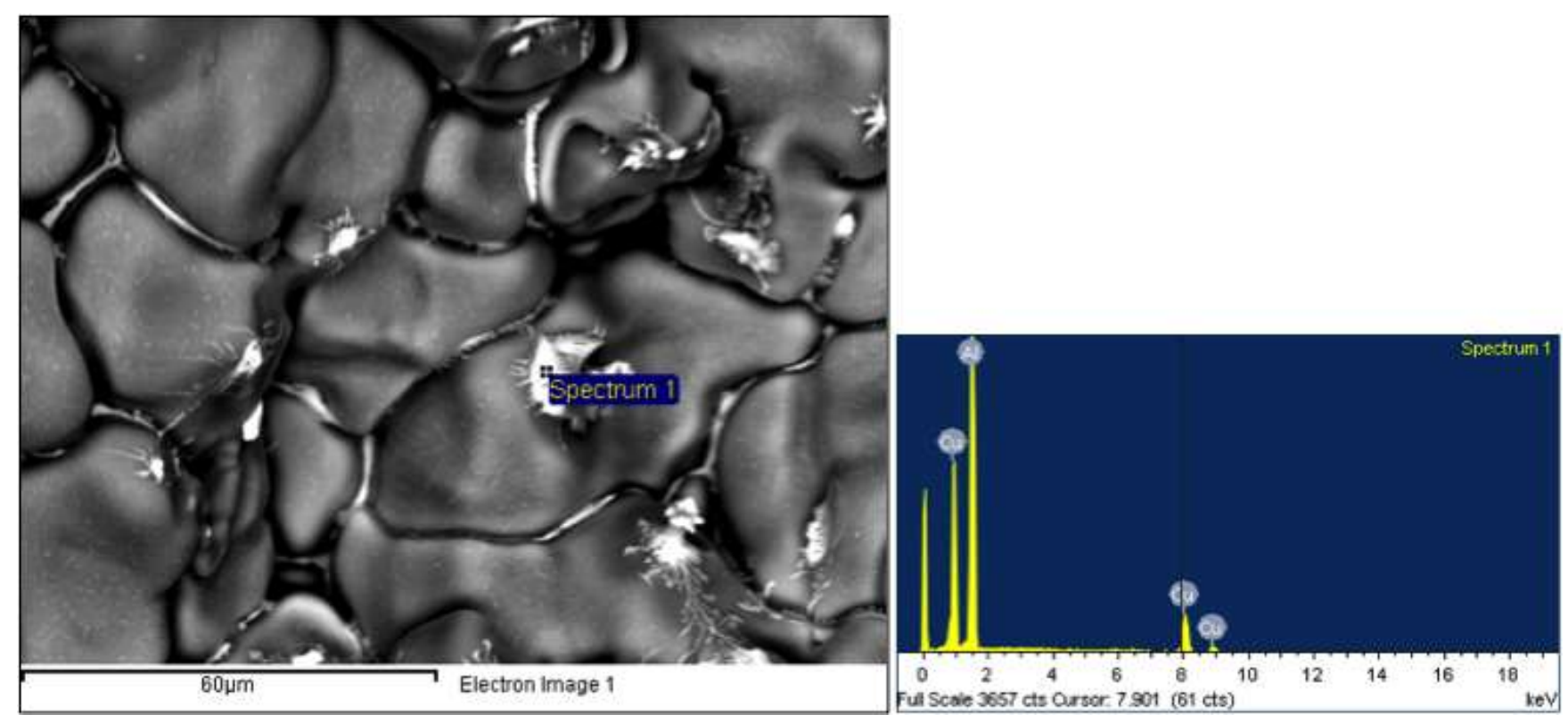

Figure 4-22. EDX analysis confirming $\mathrm{Al}_{2} \mathrm{Cu}$ along hot tear surface of unrefined $\mathrm{B} 206$.

\subsubsection{0.02wt\% Ti Alloy}

A surface hot tear was present along the top edge of the horizontal bar at the sprue-bar junction for the $0.02 \mathrm{wt} \% \mathrm{Ti}$ alloy (recall Figure 4-4). Scanning electron microscopy was carried out along this location (Sample \#1 from Figure 4-18), and the resulting micrograph is shown in Figure 4-23. The hot tear along the horizontal bar is clearly evident in the micrograph. Finer secondary tears are also seen propagating from the large tear. As was the case for the unrefined alloy, the tears propagated along grain boundary regions. Confirmation of this is given by the exposed fracture surface below the tear in Figure 4-23, as a dendritic morphology is clearly evident. A closer examination of the fracture surface in Figure 4-24 also illustrates the $\mathrm{Al}_{2} \mathrm{Cu}$ (indicated by the arrows) dispersed along the dendrites.

In comparison to the unrefined alloy, a significantly larger hot tear was present at the sprue-bar junction for the $0.02 \mathrm{wt} \% \mathrm{Ti}$ alloy. This disparity in crack size was likely attributed to the large hot tear present at the middle of the horizontal bar for the unrefined alloy. This large tear nearly separated the bar into two portions, which in turn reduced the overall (intact) length and hence 'mechanical strength' of the contracting horizontal bar. As a result, there was a lower amount of 'pulling' at the sprue-bar junction as the horizontal bar contracted towards the end restraint in the 
unrefined alloy. In contrast, the horizontal bar remained intact in the $0.02 \mathrm{wt} \% \mathrm{Ti}$ condition, and hence, a larger (intact) length increased the amount of 'pulling' at the sprue-bar junction as the horizontal bar contracted towards the end restraint. This in turn, resulted in a larger tear at this region for the $0.02 \mathrm{wt} \% \mathrm{Ti}$ alloy. Residual strain measurements presented in Section 4.5 will help to confirm this hypothesis.

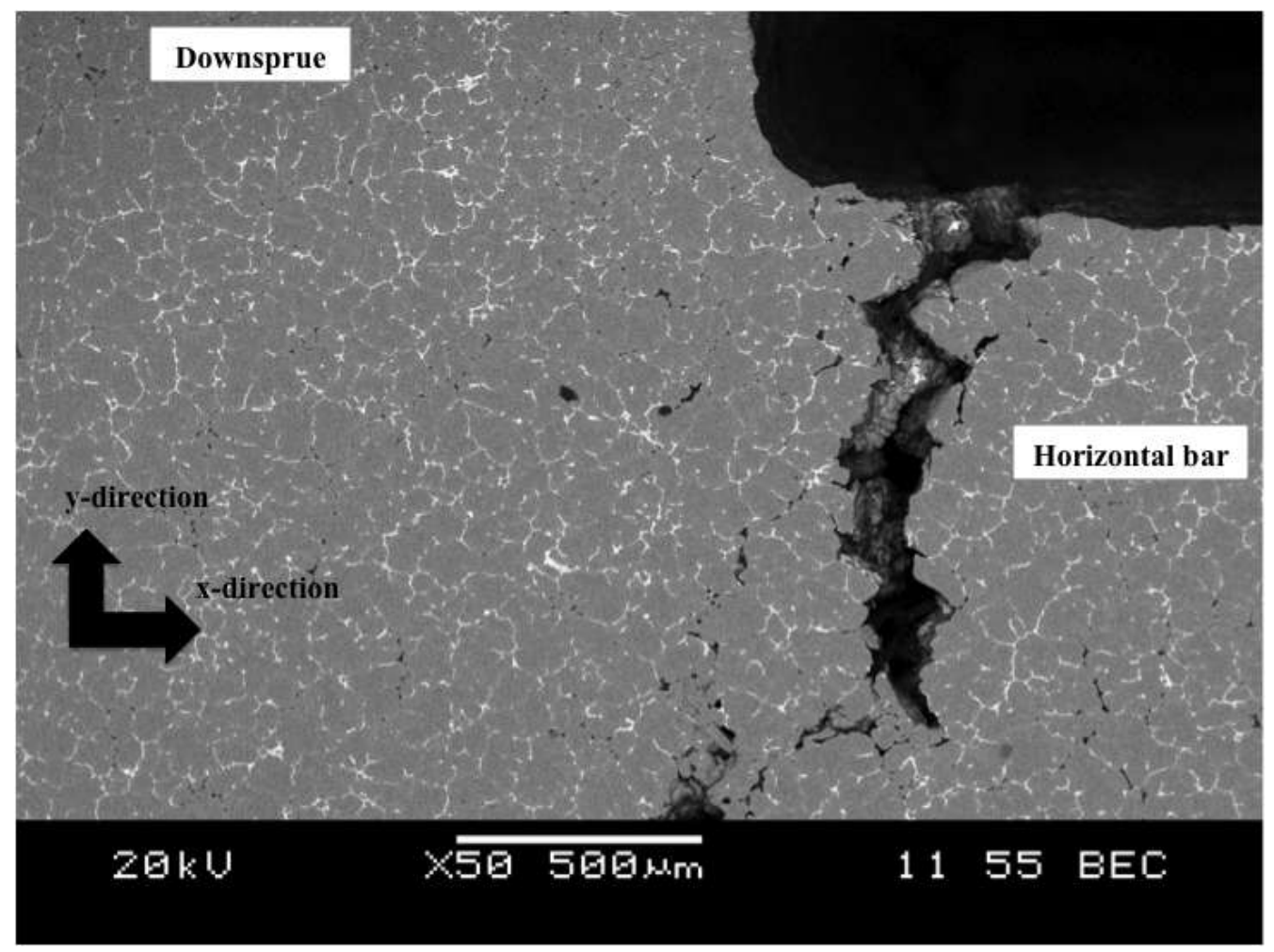

Figure 4-23. Presence of hot tear along sprue-bar junction in $0.02 \mathrm{wt} \%$ Ti alloy.

\subsubsection{0.05 wt\% Ti Alloy}

A hot tear was not seen along any of the four samples at the sprue-bar junction for the $0.05 \mathrm{wt} \% \mathrm{Ti}$ alloy. The stereomicrographs in Appendix 2 confirm this observation. Examination of the microstructure in Figure 4-25 using SEM (along Sample \#1 in Figure 4-18) reveals the presence of sporadically dispersed microcracks along regions of deformed grains. However, in contrast to the unrefined and $0.02 \mathrm{wt} \% \mathrm{Ti}$ alloys, these microcracks were not seen to develop into complete hot tears. A closer view of the deformed grains is shown in Figure 4-26. The presence of such grains suggests that the alloy's globular grain morphology resisted hot tear formation by 
effectively accommodating the strain induced by both the contracting downsprue and horizontal bar. This in turn, enhanced the ductility of the alloy and resisted the formation of hot tears. Such deformed grains were not visible on the micrographs of the unrefined and $0.02 \mathrm{wt} \% \mathrm{Ti}$ alloys, since the grains in these alloys were unable to resist deformation and subsequent fracture. A small fractured section is visible in the $0.05 \mathrm{wt} \% \mathrm{Ti}$ alloy, but this is not considered a hot tear, as there is no evident crack propagation in the tear vicinity.

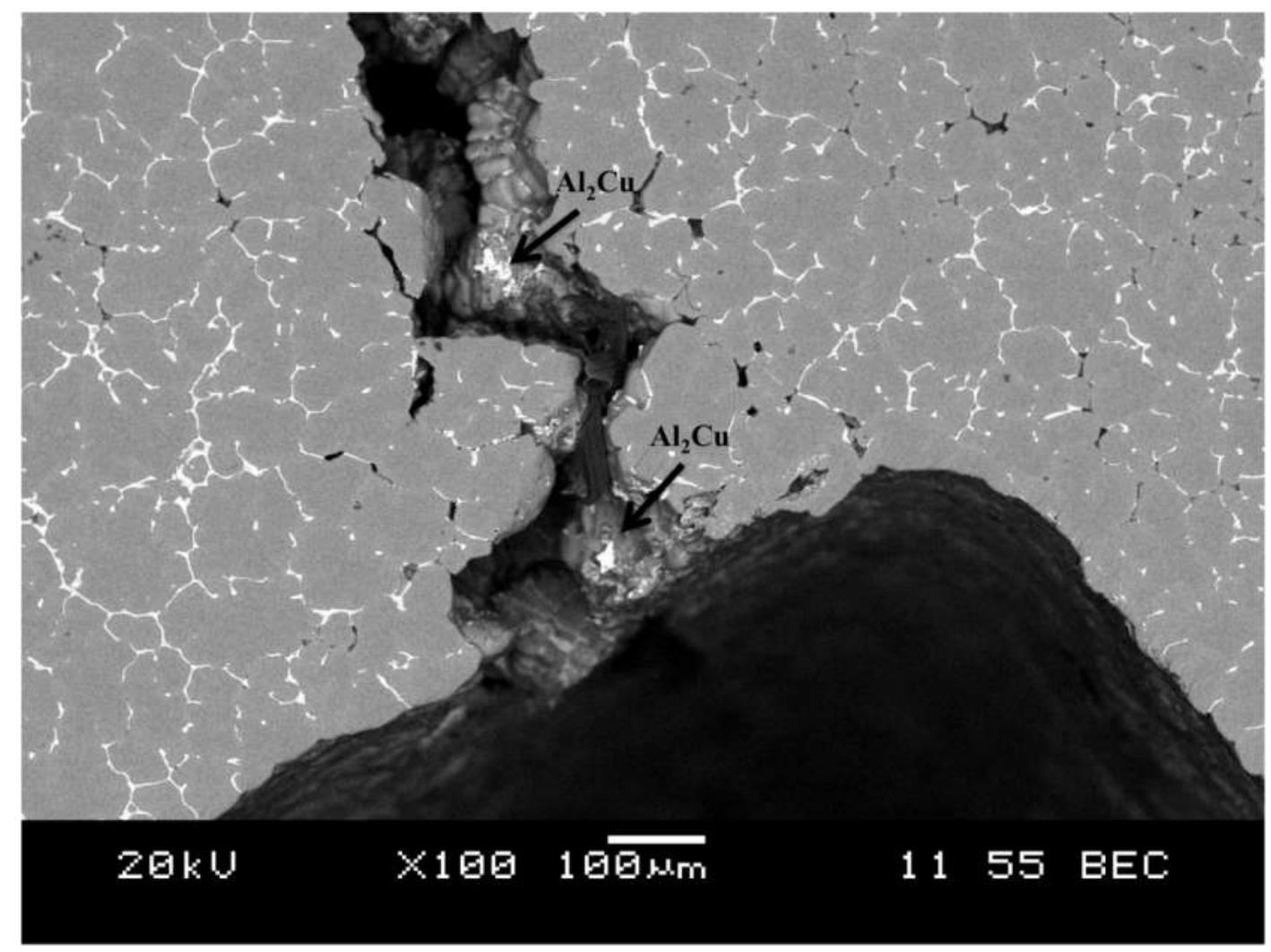

Figure 4-24. Dendritic hot tear fracture surface in $0.02 \mathrm{wt} \%$ Ti alloy. 


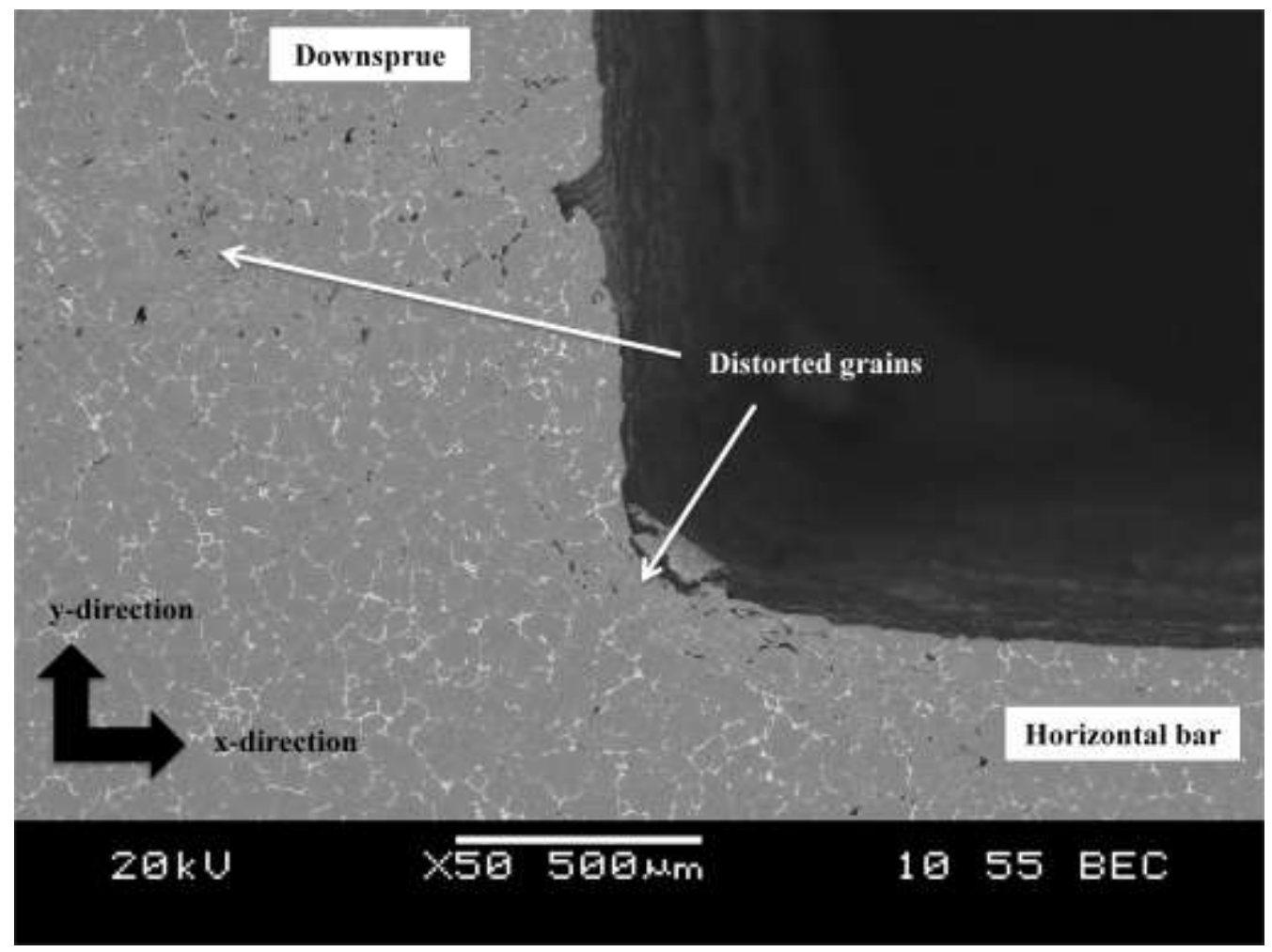

Figure 4-25. Evidence of grain deformation along sprue-bar junction in $0.05 \mathrm{wt} \%$ Ti alloy.

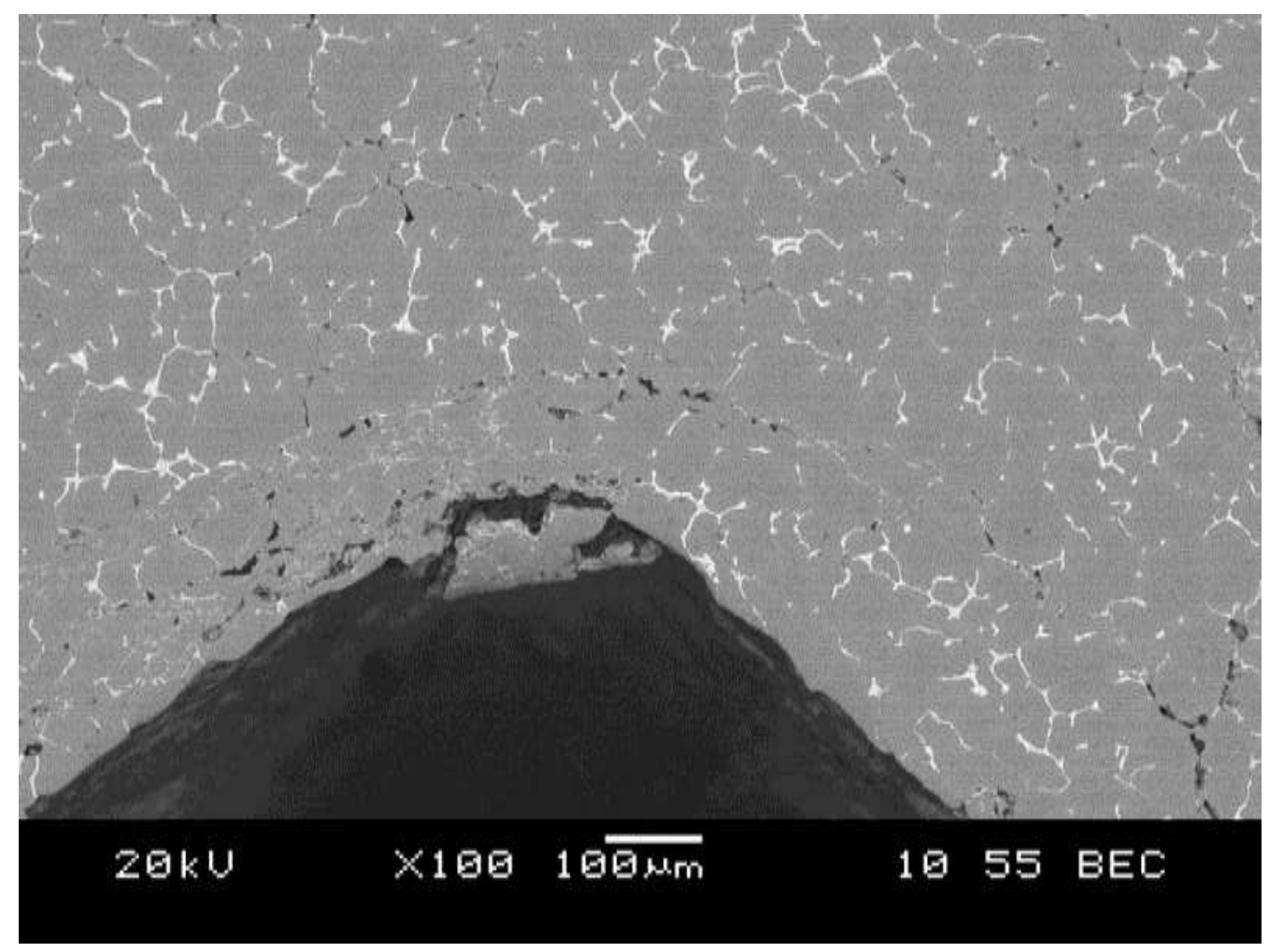

Figure 4-26. A closer view of the deformed grains in $0.05 \mathrm{wt} \% \mathrm{Ti}$ alloy. 


\subsubsection{Casting Porosity}

The percent area porosity was measured for the three B206 conditions using an image analysis software in conjunction with an optical microscope. The measurements were taken at three different locations along the horizontal bar for the three conditions. A minimum of 40 measurements was made on each sample following a square grid pattern. The results are given in Figure 4-27. Additions of Ti successfully reduced the amount of porosity in the B206 castings. This was likely a direct result of alloy grain morphology, as the coarse dendrites in the unrefined alloy limited liquid metal feeding to small regions between dendrite arms at late stages of solidification. As a result, many areas of shrinkage porosity were revealed from microstructure analysis. An example of this is illustrated in Figure 4-28. In contrast, the equiaxed grain morphology of the refined alloys promoted uniform feeding of liquid metal along grain boundary regions and improved the ability of the alloys to accommodate shrinkage during solidification and in turn, reduce the overall amount of shrinkage porosity.

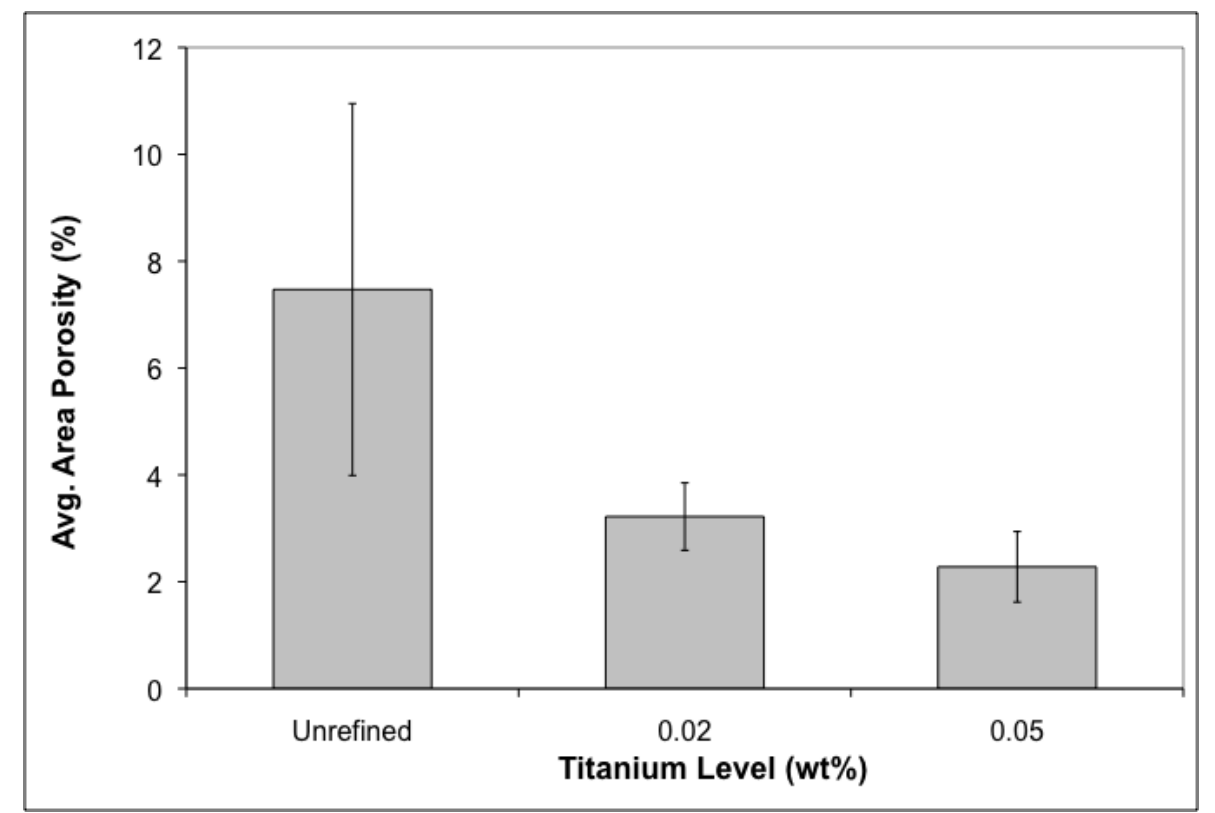

Figure 4-27. Percent area porosity measurements for B206 with various levels of Ti.

Inspection of the porosity measurements in Figure 4-27 also demonstrates a significantly greater variance (error bars) to mean ratio of porosity for the unrefined alloy in comparison to that of the refined alloys. This suggests that the distribution of porosity throughout the casting of the 
unrefined alloy was less uniform. Again, this was likely due to the respective grain morphologies of the unrefined and refined alloys. In the unrefined alloy, the coarse dendrites created both large and small regions (through dendrite arms) for late stage feeding of liquid metal to take place. In turn, liquid metal feeding was enhanced in some areas while limited in others. This resulted in a non-uniform distribution of porosity. In contrast, the equiaxed grain morphology of the refined alloys (more so the $0.05 \mathrm{wt} \% \mathrm{Ti}$ alloy) generated pockets of similar (or uniform) size along grain boundary regions and thereby promoted uniform liquid metal feeding and an improved distribution of porosity throughout the casting. This improved distribution of porosity was significant because it decreased the likelihood of hot tears propagating from regions of agglomerated porosity.

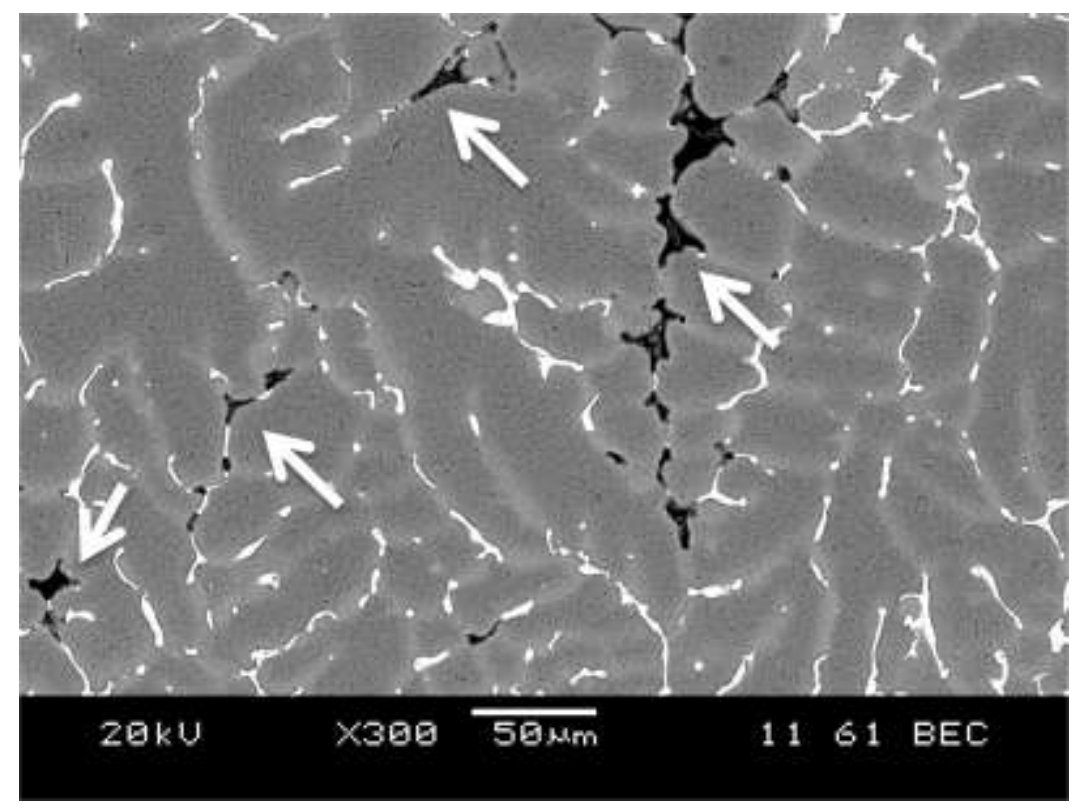

Figure 4-28. Shrinkage porosity in unrefined B206.

\section{$\underline{\text { Section Summary }}$}

Optical and scanning electron microscopy was carried out on the three B206 alloys. Additions of Ti were found to successfully reduce grain size and transform the grain morphology of B206 into a more globular structure. This resulted in a more uniform feeding of liquid metal through grain boundary regions and in turn promoted a more homogeneous distribution of $\mathrm{Al}_{2} \mathrm{Cu}$ along grain boundary regions for the refined alloys. Such improved feeding in the refined alloys enabled a reduction of overall shrinkage porosity in these alloys as well. Finally, examination of the 
sprue-bar junction demonstrated a good correlation between microstructure and hot tearing severity at this region.

\subsection{Thermal Analysis}

Thermal analysis was carried out for the three B206 casting conditions. Cooling curves (temperature vs. time) were generated for each condition along the horizontal bar of the casting (see Figure 3-4). During solidification, latent heat is released causing a change in the cooling curve of the alloy system. The evolution of heat establishes a thermal arrest point in the cooling curve [76]. An example of a cooling curve for B206 is shown in Figure 4-29. Inspection of the curve illustrates two locations where such a thermal arrest occurs: one corresponding to the start of the solidification process (point 1) and the second corresponding to the main eutectic reaction (point 2) under which intermetallic $\mathrm{Al}_{2} \mathrm{Cu}$ precipitates [77].

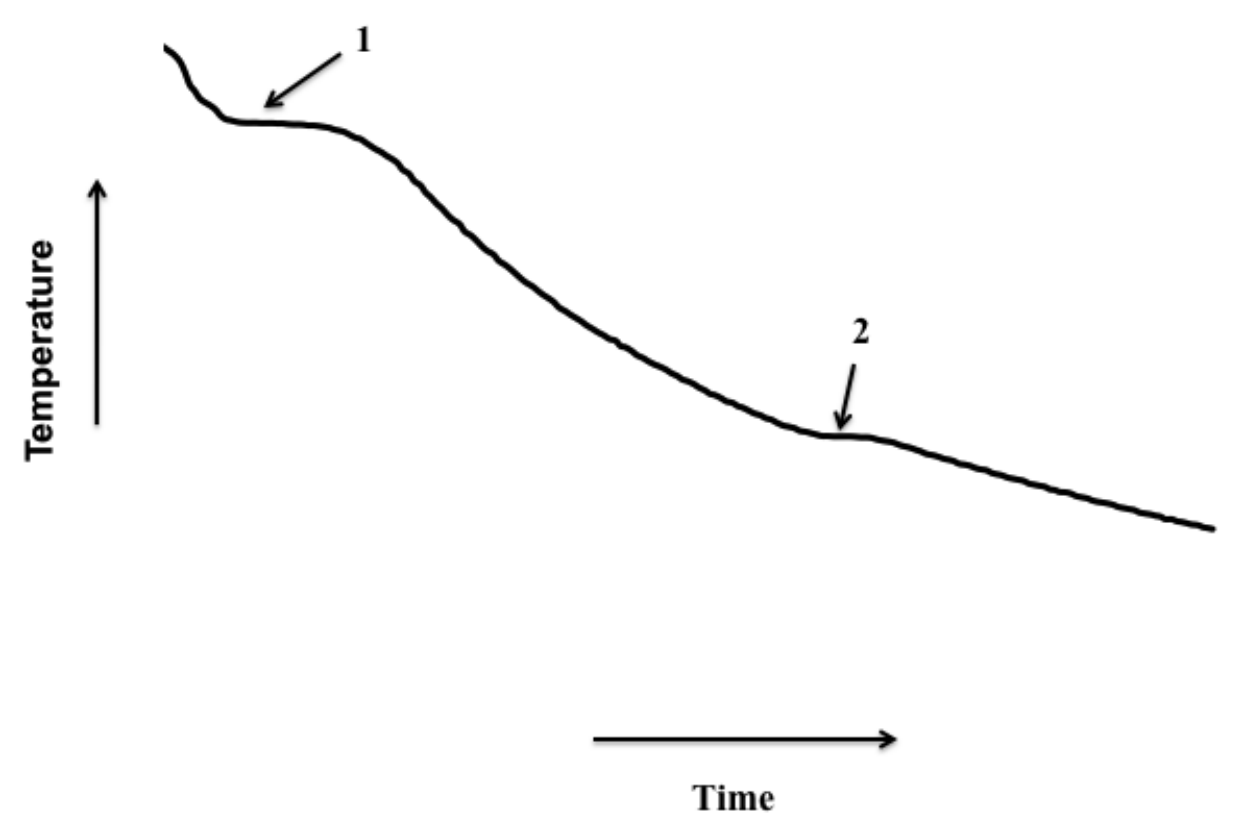

Figure 4-29. An example-cooling curve for B206. 
More detailed information on the solidification process may be obtained from the first derivative of the cooling curve. The derivative at each point of the curve is numerically equal to the slope of the cooling curve, and hence represents the rate of cooling of the alloy during solidification. An increase in the derivative signifies the occurrence of an event, which slows the rate of cooling and liberates latent heat, such as the appearance of a new phase [77]. The cooling curve and its first derivative of the three B206 casting conditions are presented and discussed hereunder. The results were generated from the thermocouple by the sprue (Figure 3-4).

\subsubsection{Unrefined B206}

The cooling curve (gray) and its first derivative (black) are shown in Figure 4-30 for the unrefined B206 alloy. Initially, the alloy was completely liquid and the maximum cooling rate was measured at $23.7^{\circ} \mathrm{C} / \mathrm{s}$. The derivative curve was then seen to climb rapidly (point 1 ) to a maximum of $+1.4{ }^{\circ} \mathrm{C} / \mathrm{s}$ before suddenly dropping again. This suggests that at the maximum derivative the alloy was actually heating up at a rate of $1.4{ }^{\circ} \mathrm{C} / \mathrm{s}$. A magnified image of this region (from -5 to $5{ }^{\circ} \mathrm{C} / \mathrm{s}$ ) of the cooling curve and first derivative curve in Figure 4-31 confirms the heating up of the alloy. This rapid heat release was likely attributed to the sudden nucleation of $\alpha$-Al grains in the alloy. In the unrefined alloy, it was necessary for the melt to undercool in order to nucleate $\mathrm{Al}$ grains. With time, the rate of nucleation subsided and growth of the $\alpha-\mathrm{Al}$ dendrites progressed from the cold mold wall towards the warm centre of the casting (region 2 of the curve). Regions 1 and 2 of the first derivative curve corresponded to the first thermal arrest point (point 1 in Figure 4-29) on the cooling curve. As solidification progressed, the growing dendrites filled the casting and began to impinge on one another, thereby limiting the remainder of dendrite growth to proceed laterally (region 3 of the curve). Recall from Section 2.1 that this is the coherency point and thus, the temperature at which this occurs (given in Table 4-2) is known as the coherency temperature. Immediately following the coherency point, a sharp peak was again observed along the first derivative curve (point 4). Literature suggests that this was likely a result of the formation of the $\mathrm{Fe} / \mathrm{Mn}$ phases $\left(\mathrm{Al}_{20} \mathrm{Mn}_{3} \mathrm{Cu}_{2}\right.$ and/or $\mathrm{Al}_{6}(\mathrm{MnFeCu})$ [77]. This phase was visible in the microstructure (see Figure 4-16). Such a point was not detectable on the cooling curve of the alloy. Beyond this point, the $\alpha$-Al dendrites continued to grow and thicken as depicted by region 5 of the first derivative curve. Finally, another rapid increase in the 
derivative was detected (point 6), which corresponded to the nucleation of $\mathrm{Al}_{2} \mathrm{Cu}$. The temperature at which this occurred is given in Table 4-2 and may be taken as the end of solidification (i.e. non-equilibrium solidus temperature) for B206.

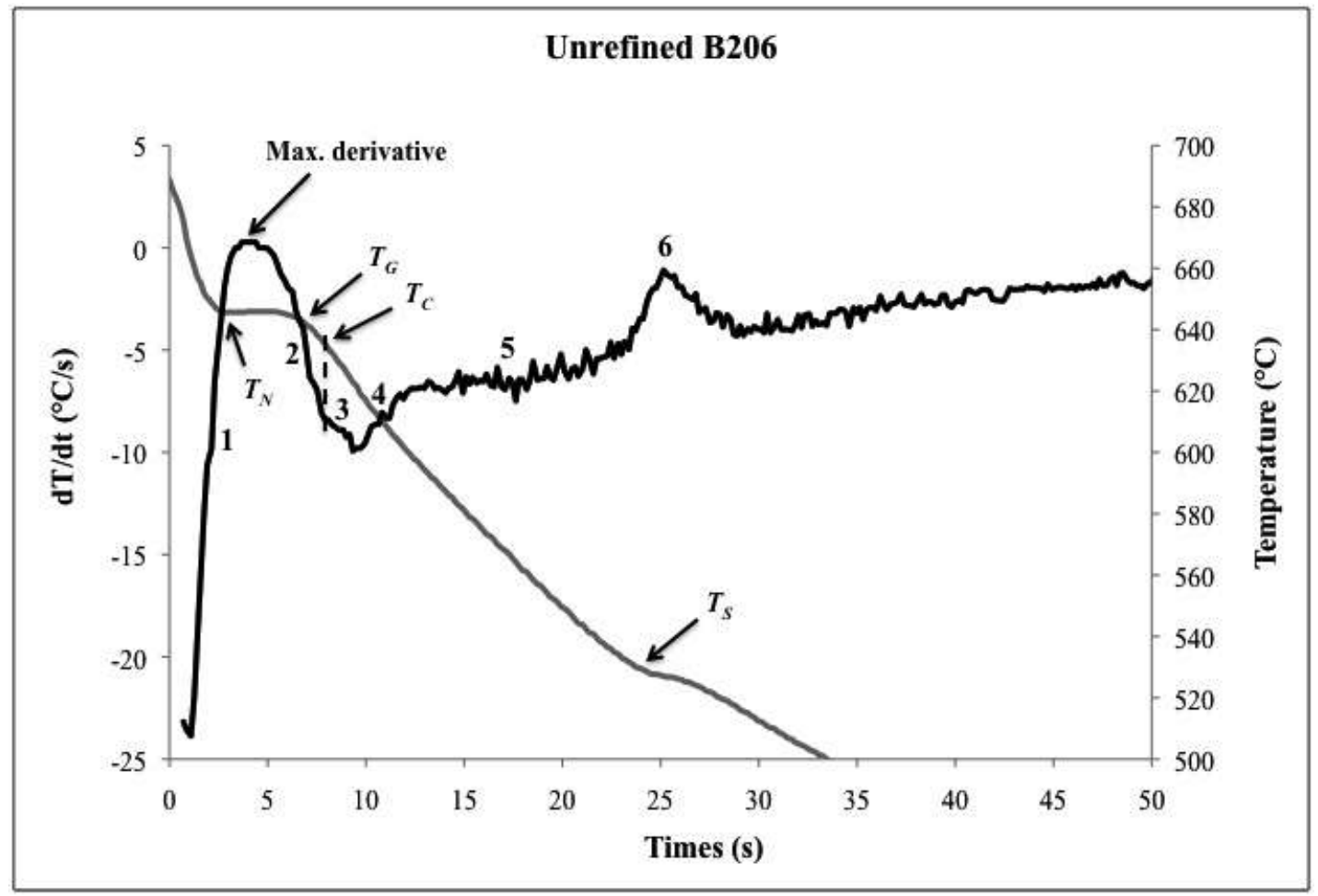

Figure 4-30. Cooling curve and its first derivative for the unrefined B206 alloy.

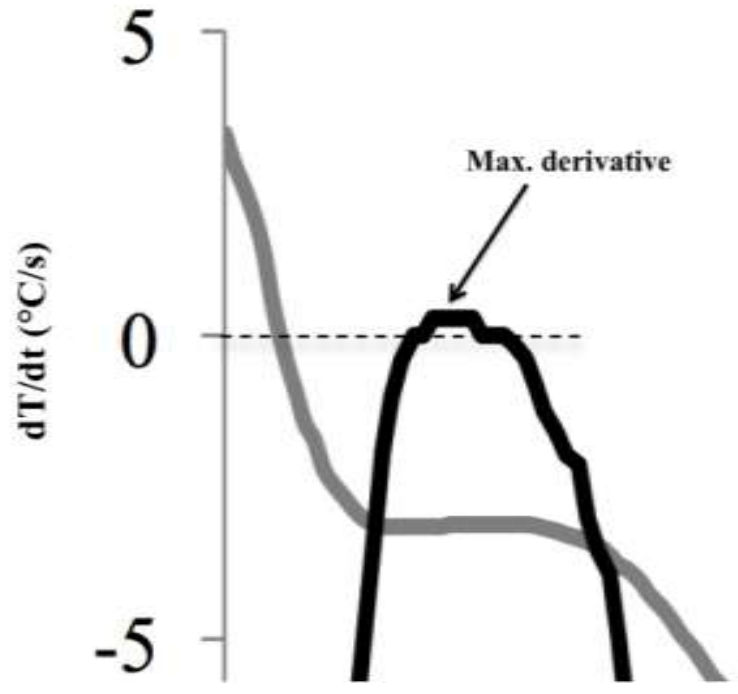

Figure 4-31. Evidence of undercooling in first derivative curve of unrefined B206. 


\subsubsection{Refined Alloys}

The cooling and first derivative curves of the two refined alloys are depicted in Figures 4-32 and 4-33. The maximum cooling rates were recorded as $21.4{ }^{\circ} \mathrm{C} / \mathrm{s}$ and $16.3{ }^{\circ} \mathrm{C} / \mathrm{s}$ for the $0.02 \mathrm{wt} \% \mathrm{Ti}$ and $0.05 \mathrm{wt} \%$ Ti alloys, respectively. The peak corresponding to the nucleation of $\mathrm{Al}$ grains was examined at high magnification for these alloys as well. Figure 4-34 shows that in contrast to the unrefined alloy, undercooling of the melt was not required to nucleate $\mathrm{Al}$ grains in the $0.02 \mathrm{wt} \% \mathrm{Ti}$ alloy, as the maximum of the first derivative curve did not exceed $0{ }^{\circ} \mathrm{C} / \mathrm{s}$. A similar result was found for the $0.05 \mathrm{wt} \% \mathrm{Ti}$ alloy as well. This suggests that additions of Ti particles (i.e. $\mathrm{TiB}_{2}$ and $\mathrm{TiAl}_{3}$ ) via the $\mathrm{Al}-5 \mathrm{Ti}-1 \mathrm{~B}$ master alloy were effective at nucleating $\alpha$-Al grains in B206, as confirmed by the presence of $\mathrm{Ti}$ in the middle of grains Figure 4-17 (Section 4.3.3). The remainder of the derivative curve (points 2 to 6) followed a similar pattern to that of the unrefined alloy. A summary of the temperatures, $T$ and time, $t$ recorded from these regions are presented and discussed in the following section.

\subsubsection{Solidification Characteristics of B206}

A summary of the results from thermal analysis is given in Tables 4-2 and 4-3 for the three B206 conditions. From Table 4-2, it was found that addition of $\mathrm{Ti}$ significantly increased the nucleating temperature, $T_{N}$ in the B206 alloy. Further, in the refined alloys, $T_{N}$ was found to be higher than the growth temperature, $T_{G}$. This was a direct result of the nucleating particles present in the Al-5Ti-1B master alloy, which effectively nucleated Al grains [4]. In contrast, undercooling of the liquid was necessary to nucleate $\mathrm{Al}$ grains in the unrefined alloy and in turn, $T_{N}$ was lower than $T_{G}$. The coherency temperature was also affected by Ti additions, as the temperature was seen to decrease with increasing Ti. Hence, grain refinement was successful in delaying coherency in B206. The remaining events (i.e. $\mathrm{Al}_{2} \mathrm{Cu}$ precipitation and the end of solidification, $T_{S}$ ) were found to occur at similar temperatures for the three conditions. The time intervals for which these events took place are presented in Table 4-3 and discussed hereunder. 


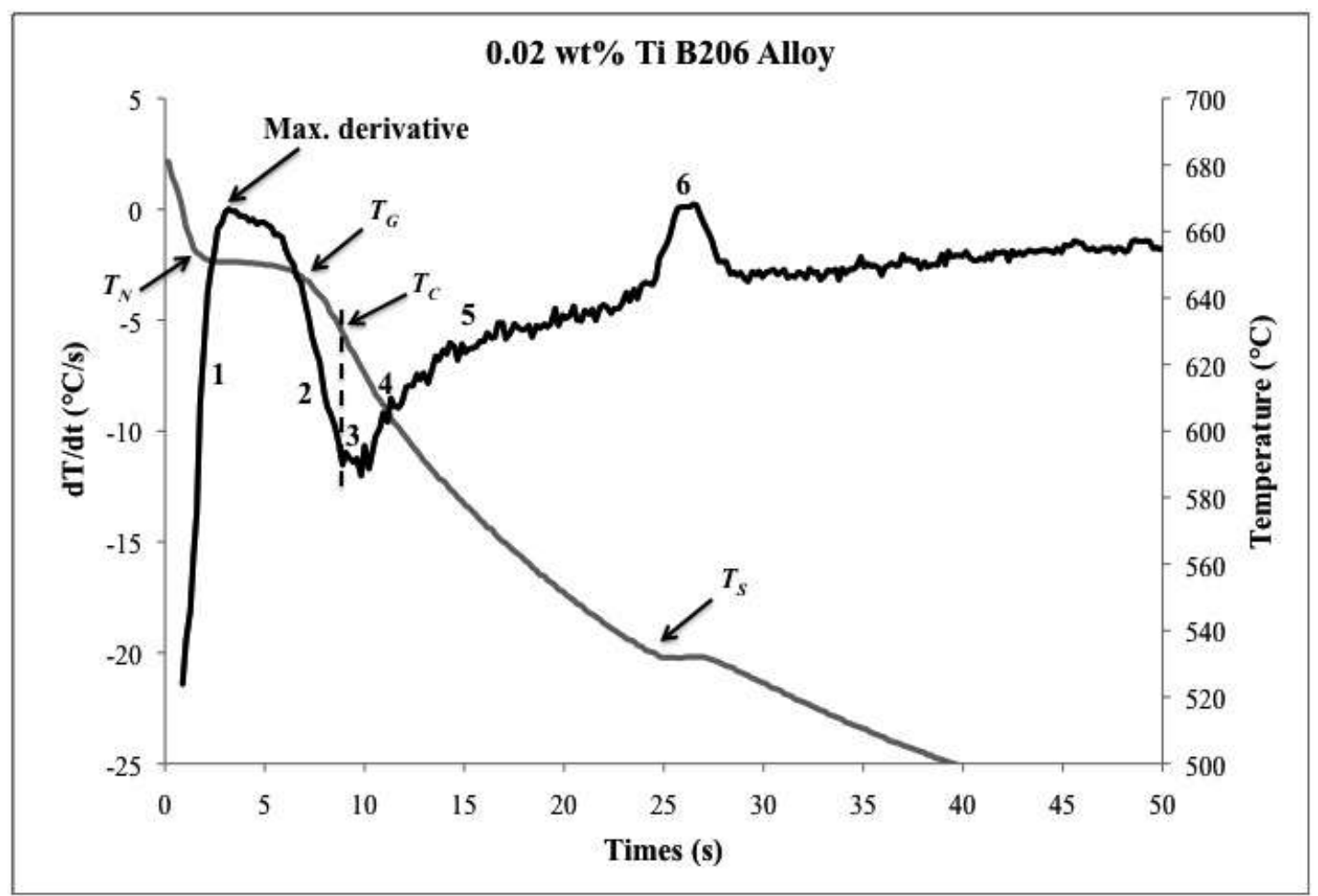

Figure 4-32. Cooling curve and its first derivative for the $0.02 \mathrm{wt} \%$ Ti alloy.

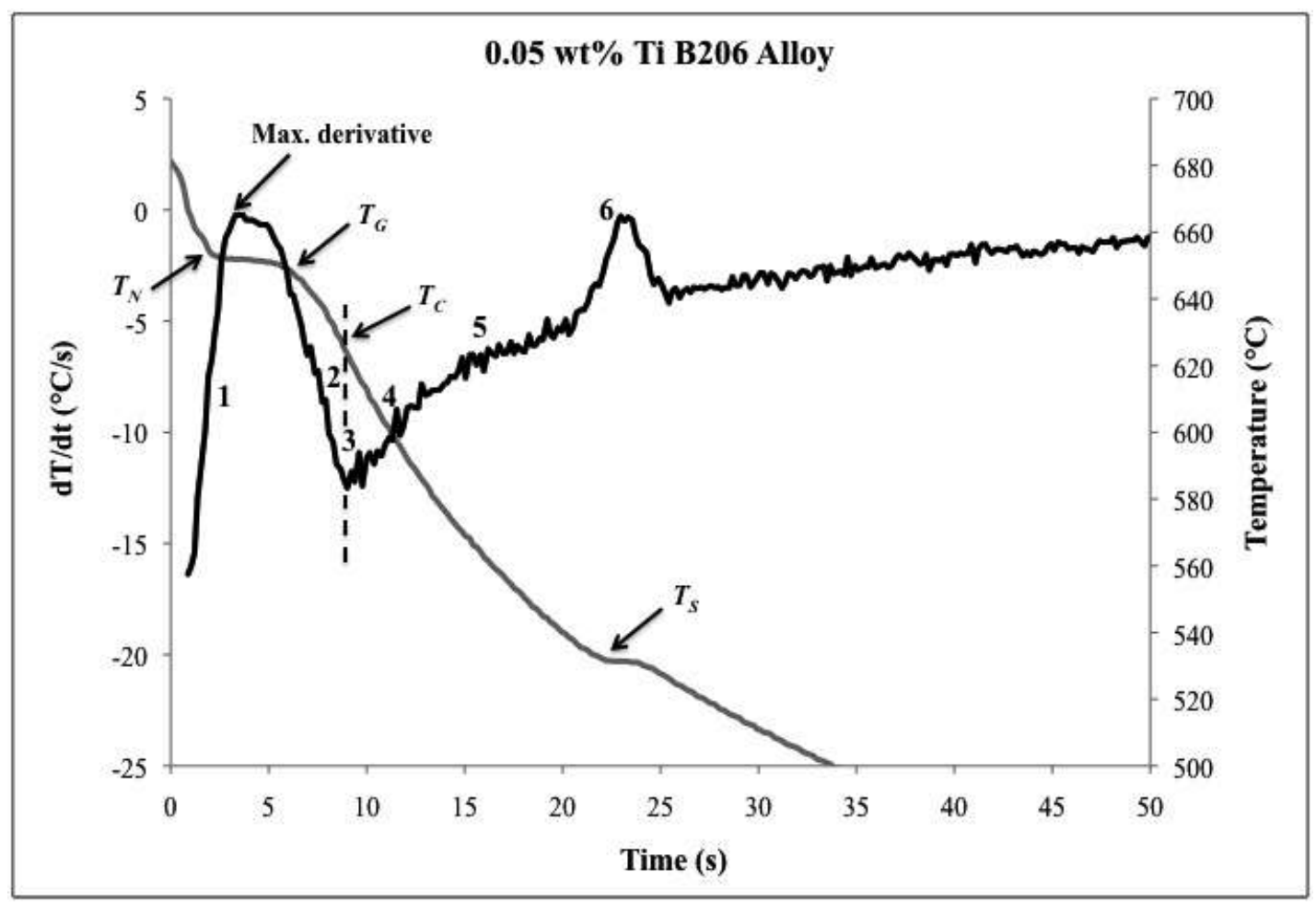

Figure 4-33. Cooling curve and its first derivative for the $0.05 \mathrm{wt} \%$ Ti alloy. 


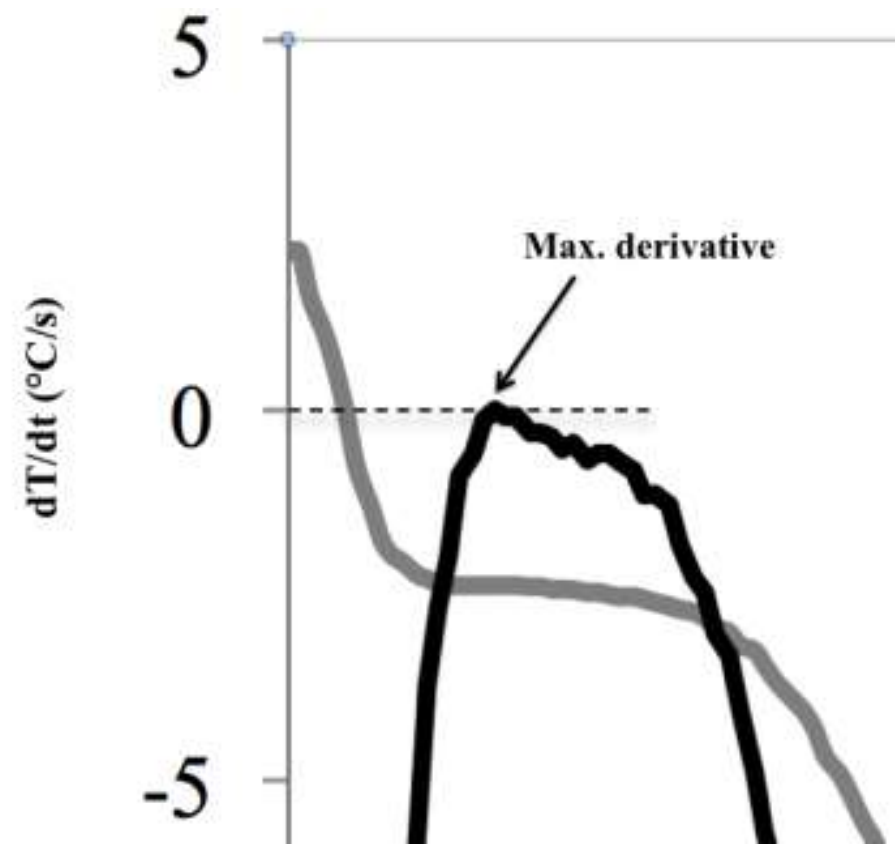

Figure 4-34. Evidence of no undercooling in first derivative curve of the $0.02 \mathrm{wt} \%$ Ti alloy.

Table 4-2. Thermal analysis data (by the sprue-bar junction).

\begin{tabular}{|c|c|c|c|c|c|c|c|c|}
\hline \multirow{2}{*}{ Condition } & \multicolumn{2}{|c|}{ Nucleation } & \multicolumn{2}{c|}{ Growth } & \multicolumn{2}{c|}{ Coherency } & \multicolumn{2}{c|}{ Solidification } \\
\cline { 2 - 9 } & $\begin{array}{c}\boldsymbol{T}_{\boldsymbol{N}} \\
\left({ }^{\circ} \mathbf{C}\right)\end{array}$ & $\begin{array}{c}\boldsymbol{t}_{\boldsymbol{N}} \\
(\mathbf{s})\end{array}$ & $\begin{array}{c}\boldsymbol{T}_{\boldsymbol{G}} \\
\left({ }^{\circ} \mathbf{C}\right)\end{array}$ & $\begin{array}{c}\boldsymbol{t}_{\boldsymbol{G}} \\
(\mathbf{s})\end{array}$ & $\begin{array}{c}\boldsymbol{T}_{\boldsymbol{C}} \\
\left({ }^{\circ} \mathbf{C}\right)\end{array}$ & $\begin{array}{c}\boldsymbol{t}_{C} \\
(\mathbf{s})\end{array}$ & $\begin{array}{c}\boldsymbol{T}_{\boldsymbol{S}} \\
\left({ }^{\circ} \mathbf{C}\right)\end{array}$ & $\begin{array}{c}\boldsymbol{t}_{\boldsymbol{S}} \\
(\mathbf{s})\end{array}$ \\
\hline Unrefined & 645.5 & 3.0 & 646.0 & 5.5 & 635.5 & 7.0 & 531.0 & 23.5 \\
\hline $0.02 \mathrm{wt} \% \mathrm{Ti}$ & 653.0 & 1.5 & 650.0 & 5.0 & 628.0 & 9.0 & 533.0 & 24.5 \\
\hline $0.05 \mathrm{wt} \% \mathrm{Ti}$ & 655.0 & 2.0 & 650.5 & 5.5 & 623.5 & 9.5 & 532.5 & 22.0 \\
\hline
\end{tabular}

Table 4-3. Differences in time at points of interest.

\begin{tabular}{|c|c|c|}
\hline \multirow{2}{*}{ Condition } & Mass feeding & LST \\
\cline { 2 - 3 } & $\boldsymbol{t}_{\boldsymbol{C}}-\boldsymbol{t}_{\boldsymbol{N}}(\mathbf{s})$ & $\boldsymbol{t}_{\boldsymbol{S}}-\boldsymbol{t}_{\boldsymbol{N}}(\mathbf{s})$ \\
\hline Unrefined & 4.0 & 20.5 \\
\hline $0.02 \mathrm{wt} \% \mathrm{Ti}$ & 7.5 & 23.0 \\
\hline $0.05 \mathrm{wt} \% \mathrm{Ti}$ & 7.5 & 20.0 \\
\hline
\end{tabular}


$\underline{t_{C}}-\underline{t_{N}}$

This time interval represents the duration of mass feeding of liquid metal during solidification of the alloys. Recall from Section 2.1 that mass feeding occurs when crystals flow freely in the melt. Hence, a higher value of $t_{C}-t_{N}$ is desirable as it suggests extended feeding of the casting by low viscosity liquid.

The addition of Ti was found to nearly double the duration of mass feeding in B206. This result confirms the findings in literature (recall Section 2.2.2.2), which suggest that grain refinement delays the onset of dendrite coherency [41-44]. Further, the increase in $t_{C}-t_{N}$ supports the observed improved castability of the refined alloys with respect to the unrefined alloy (Section 4.2). With an increased value of $t_{C}-t_{N}$, the refined alloys filled a greater portion of the casting before the grains became coherent. This improvement in bulk feeding likely delayed the onset of solidification stresses during solidification and in turn, may have decreased the overall magnitude of stress for the refined alloys. Moreover, in agreement with the theory proposed by Clyne and Davies [21], such increased time of mass feeding for the refined alloys would result in lower CSC values (Equation 2) for the refined alloys and thus improved hot tearing resistance.

The $t_{C}-t_{N}$ parameter was used to estimate the dendrite (or grain) growth velocity for the three conditions of B206. From the average grain sizes in Table 4-1 (Section 4.3.2), the average growth velocity was calculated. The results in Table 4-4 clearly illustrate the rapid dendrite growth in the unrefined alloy in comparison to that of the refined alloys. Such rapid growth in the unrefined alloy may have been due to the undercooling that occurred during solidification of the alloy. The immediate increase in temperature (and subsequent release of heat) upon nucleation of $\alpha-\mathrm{Al}$ grains (i.e. recalescence) likely spurred rapid growth of dendrites. In contrast, the addition of Ti to B206 was effective at slowing grain growth by generating many nucleating sites (i.e. no undercooling) and thereby increasing the number of grains within the casting.

$\underline{t_{S}} \underline{-} \underline{\underline{t}} \underline{\underline{N}}$

This time interval represents the local solidification time (LST) of the alloys. The results in Table 4-3 suggest that LST was unaffected by Ti additions, as similar values were seen for each condition. The LST was therefore likely solely influenced by mold temperature. 
Table 4-4. Average dendrite/grain growth velocity in B206.

\begin{tabular}{|c|c|}
\hline Condition & $\begin{array}{c}\text { Growth Velocity } \\
(\boldsymbol{\mu m} / \mathbf{s})\end{array}$ \\
\hline Unrefined & 266 \\
\hline $0.02 \mathrm{wt} \% \mathrm{Ti}$ & 14 \\
\hline $0.05 \mathrm{wt} \% \mathrm{Ti}$ & 10 \\
\hline
\end{tabular}

The LST was also measured at two other locations along the horizontal bar: at the middle and by the end restraint (Figure 3-4). The results are presented in Table 4-5 for the three conditions. The remaining thermal analysis data at these regions is presented in Appendix 3 (Tables A.3-1 to A.3-4). The purpose of these measurements was to examine whether any differences in solidification occurred along the horizontal bar for each condition in order to gain a better understanding of the solidification profile. The middle of the horizontal bar was seen to be the last region to solidify, as the LST was highest at this region for all conditions. As a result, this suggested that the middle of the horizontal bar remained the hot spot region during solidification and therefore was most prone to hot tear formation. This was in agreement to the location of the hot tear for the unrefined alloy (Figure 4-1).

Table 4-5. LST at middle and end of horizontal bar.

\begin{tabular}{|c|c|c|}
\hline \multirow{2}{*}{ Condition } & \multicolumn{2}{|c|}{$\mathbf{t}_{\mathbf{S}}-\mathbf{t}_{\mathbf{N}}(\mathbf{s})$} \\
\cline { 2 - 3 } & Middle & End \\
\hline Unrefined & 22.0 & 19.5 \\
\hline $0.02 \mathrm{wt} \% \mathrm{Ti}$ & 25.5 & 21.0 \\
\hline $0.05 \mathrm{wt} \% \mathrm{Ti}$ & 24.5 & 19.0 \\
\hline
\end{tabular}

\section{Section Summary}

The solidification characteristics of B206 were examined using thermal analysis. The results were in agreement with alloy microstructure, as it was confirmed that $\mathrm{Ti}$ was effective at nucleating $\alpha-\mathrm{Al}$ grains in B206, since no undercooling was observed during solidification of the refined alloys. Further, the onset temperature and time of coherency was delayed by grain 
refinement. This was significant in improving the overall bulk feeding of liquid metal during casting of the refined alloys and in turn, suggested that more time was available for stress accommodation by liquid feeding to occur during solidification of these alloys. Neutron diffraction strain mapping results presented in the following section illustrates the effect of such enhanced feeding on overall residual strain and stress. Lastly, thermal analysis along the horizontal bar confirmed the middle of the horizontal bar as the hot spot region. This was consistent with the location of the large hot tear on the casting surface of the unrefined alloy.

\subsection{Neutron Diffraction Residual Strain Mapping}

Additions of $\mathrm{Ti}$ effectively reduced hot tearing severity by transforming the alloy grain morphology into a finer and more globular structure. The effect of such grain refinement on the residual strain is presented for the unrefined, $0.02 \mathrm{wt} \%$ Ti and $0.05 \mathrm{wt} \%$ Ti B206 alloy castings. The residual strain profiles of each casting along the $x$-direction are presented in Section 4.5.1, while results of strain mapping for the $y$ and $z$-directions are presented in the following two sections, respectively. Lastly, the chapter concludes with the results of residual stress along the B206 castings.

\subsubsection{Residual Strain in the $x$-Direction}

The residual strain in the $x$-direction played a significant role in the formation of hot tears in B206. The strain in this direction was a result of the combined effect of the $90^{\circ}$ corner at the sprue-bar junction and end restraint, which both restricted free contraction of the horizontal bar. As a result, the profiles for the strain along this direction are divided into two separate sections: at the sprue-bar junction and along the remainder of the horizontal bar.

\subsubsection{Strain At the Sprue-Bar Junction}

The profiles of residual $x$-direction strain $\left(\varepsilon_{x}\right)$ at the sprue-bar junction are shown in Figures 4-35 to 4-37 for the three B206 casting conditions. The measurements are presented for the (111) reflection along the top and bottom edge and centreline of the horizontal bar. Strain 
measurements were also carried out along the top and bottom edge for the (311) reflection and the profiles are given in Appendix 4. The abscissa in the plots represents the distance (in $\mathrm{mm}$ ) along the horizontal bar. The $90^{\circ}$ corner at the sprue-bar junction corresponded to a location of $x=0 \mathrm{~mm}$. Residual strain measurements were initiated $\sim 20 \mathrm{~mm}$ into the downsprue (i.e. at $x \sim-20 \mathrm{~mm}$ ) and the results are presented up to $40 \mathrm{~mm}$ along the horizontal bar (i.e. at $x=40 \mathrm{~mm}$ ). The error bars in the plots represent the uncertainty in strain measurements. The methodology used to determine the uncertainty is provided in Appendix 4.

Inspection of the strain profiles along this region revealed a similar trend of strain discontinuity for the three B206 castings. Upon crossing the $90^{\circ}$ corner (at $x=0 \mathrm{~mm}$ ), the strain along the bottom edge was seen to increase to a tensile state, while the strain along the top edge decreased further into compression (or lower magnitude tension). The magnitude of strain along the centreline was generally found to lie in between those of the top and bottom edges for each alloy. The discontinuity in strain between these locations along the horizontal bar can be attributed to thermal strains generated from the expected filling sequence of the horizontal bar, along with mechanical strains generated from the shrinking downsprue and horizontal bar.

The horizontal bar was expected to solidify from the bottom edge upwards towards the top edge. This was confirmed by MAGMASOFT ® simulation for the AZ91 alloy in a previous study [57]. As the molten alloy filled the horizontal bar, the temperature difference between the mold wall and the molten B206 (i.e. $380{ }^{\circ} \mathrm{C}$ vs. $720{ }^{\circ} \mathrm{C}$ ) created a 'chilling effect', thereby forming a thin skin along the bottom edge. As this bottom edge skin cooled, it began to contract and press against the centreline regions. In order to maintain force equilibrium, the centreline region resisted the compression of the bottom edge and therefore, induced tensile strain along the bottom edge. This process repeated itself for the contracting centreline region and top edge region. As a result, the highest tensile strain was generated along the bottom edge. In contrast, the top edge was the last region to solidify and was free to contract, thereby generating compressive strains.

Mechanical strains were generated at the $90^{\circ}$ junction between the horizontal bar and downsprue. These mechanical strains also likely played a role in the strain discontinuity at the $90^{\circ}$ junction. 
Under ideal conditions, once the casting cavity was filled and solidification commenced, the downsprue would tend to contract towards the pouring cup (placed on top of the mold). In contrast, the horizontal bar would contract towards the end restraint. However, the downsprue and horizontal bar were connected at the $90^{\circ}$ corner and therefore mutually influenced each others' contraction. The tendency of the downsprue to contract towards the top of the mold and the horizontal bar to contract towards the end restraint, created a bending moment at the $90^{\circ}$ corner. This bending moment pulled the bottom edge of the horizontal bar towards the top edge, resulting in tensile strain at this location. In contrast, the top edge of the horizontal bar was pushed against the mold wall. Consequently, the reaction of the mold wall towards the top edge resulted in compressive (or lower magnitude tensile) strains at this location.

The profiles of $\varepsilon_{x}$ showed good agreement to the microstructure of the three castings at this location (Section 4.3.4). Comparison of the profiles for the unrefined alloy (Figure 4-35) and $0.02 \mathrm{wt} \% \mathrm{Ti}$ alloy (Figure 4-36) reveal higher magnitude of $\varepsilon_{x}$ along the $0.02 \mathrm{wt} \%$ Ti alloy for all three locations. This was consistent with the severity of hot tearing at this location. Further, the higher tensile strain confirms the hypothesis proposed in Section 4.3.4, as such tension likely resulted from the 'pulling' of the greater intact length of the horizontal bar for the $0.02 \mathrm{wt} \% \mathrm{Ti}$ alloy. In turn, this resulted in the formation of a more severe hot tear for the $0.02 \mathrm{wt} \% \mathrm{Ti}$ alloy. Moreover, the greater extent of cracking in the $0.02 \mathrm{wt} \% \mathrm{Ti}$ alloy, also generated a higher magnitude of strain relief along the top edge, as confirmed in Figure 4-36a. In the case of the unrefined alloy, the shorter intact length of the horizontal bar reduced the amount of 'pulling' at the sprue-bar junction and the strain remained in compression along the top edge. Nevertheless, despite such compression, microcracks formed at this region for the unrefined alloy. However, the cracks were present along the downsprue portion of the horizontal bar (i.e. at $x<0 \mathrm{~mm}$ in Figure 4-35a) which was in tension.

In the case of the refined alloys, similar profiles of tensile $\varepsilon_{x}$ were seen along the three locations (i.e. top edge, bottom edge and centreline) for both alloys. Such tension further confirms the greater magnitude of 'pulling' initiated by the intact contracting horizontal bar for the refined alloys. However, as demonstrated in Section 4.3.4, the globular grain morphology of the $0.05 \mathrm{wt} \%$ Ti alloy likely increased the strength of the alloy which in turn, enhanced its ability to 
resist such high tension. As a result, the $0.05 \mathrm{wt} \% \mathrm{Ti}$ alloy showed higher resistance to hot tearing than the $0.02 \mathrm{wt} \% \mathrm{Ti}$ alloy. Further, the larger hot tear along the top edge of the $0.02 \mathrm{wt} \%$ Ti alloy was consistent with the extent of strain relief observed along the alloy's strain profile (Figure 4-36a). In contrast, less relief was observed at the same location along the profile of the $0.05 \mathrm{wt} \%$ Ti alloy (Figure 4-37a).

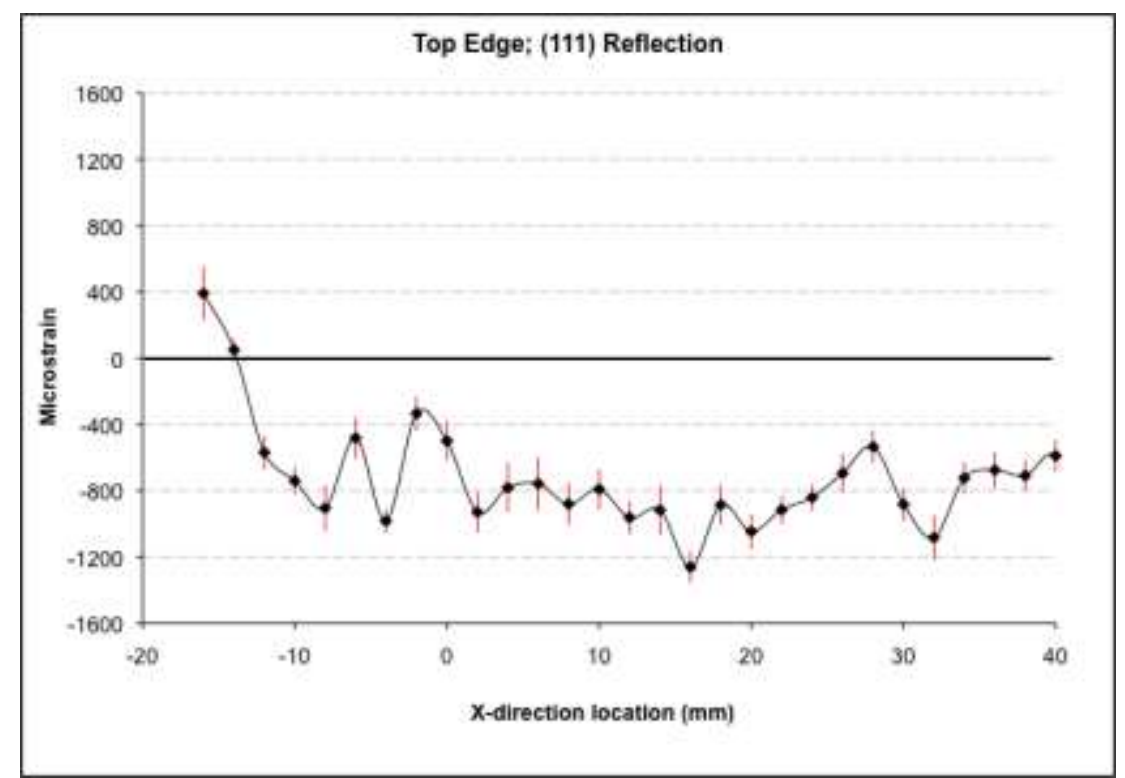

a)

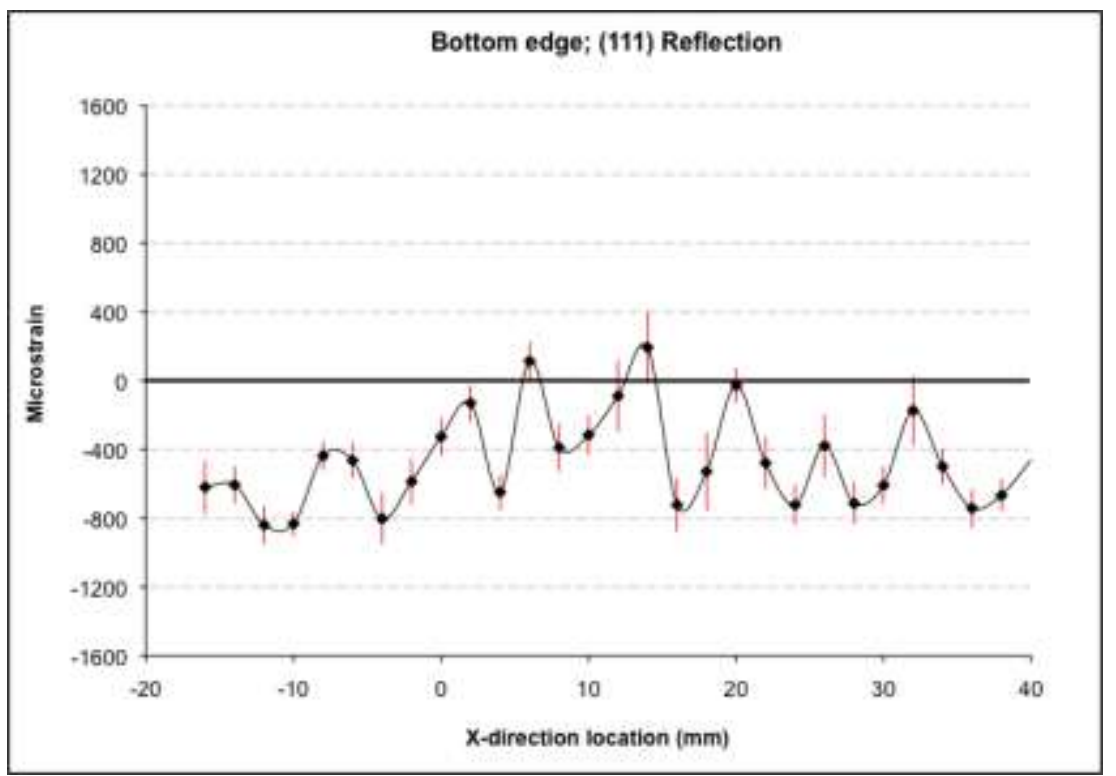

b) 


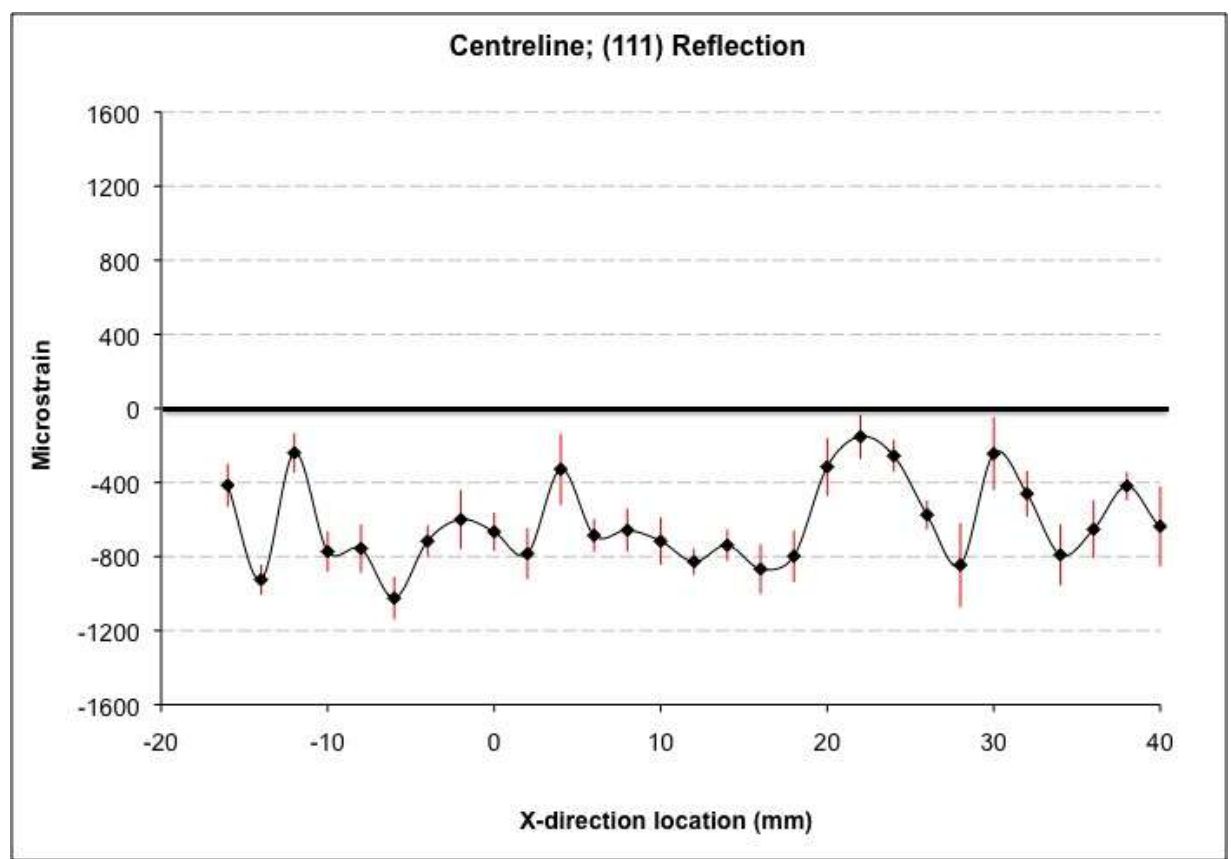

c)

Figure 4-35. Profiles of $\varepsilon_{x}$ at the sprue-bar junction along the a) top edge; b)bottom edge and c) centreline of the unrefined alloy's horizontal bar for the (111) reflection.

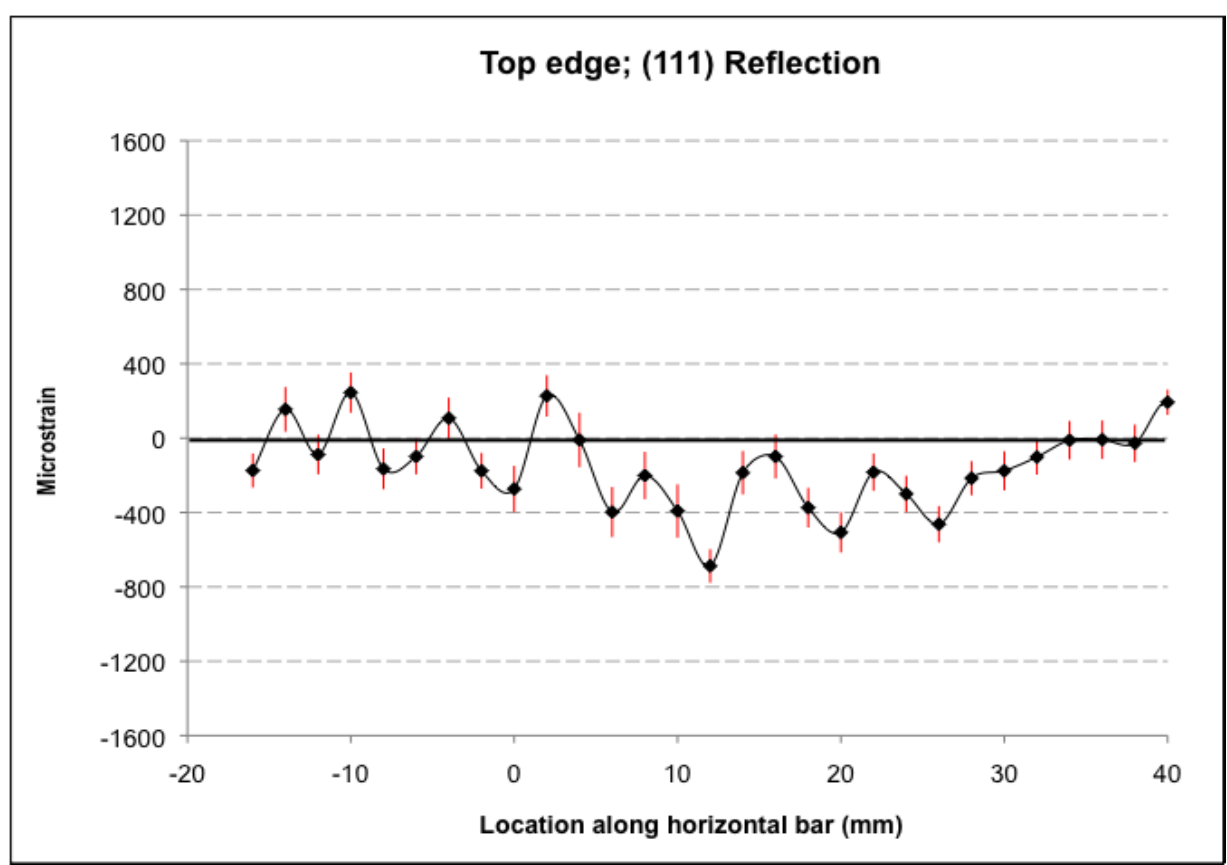

a) 


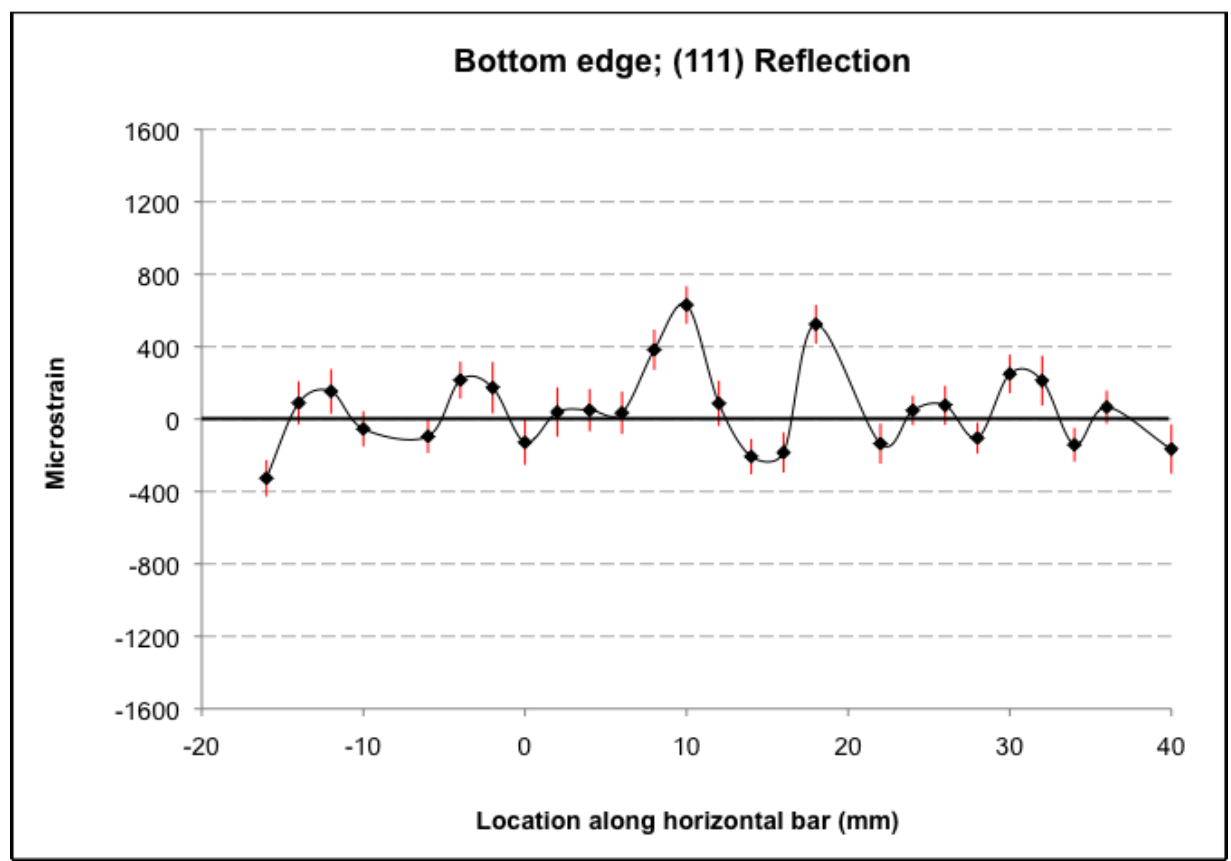

b)

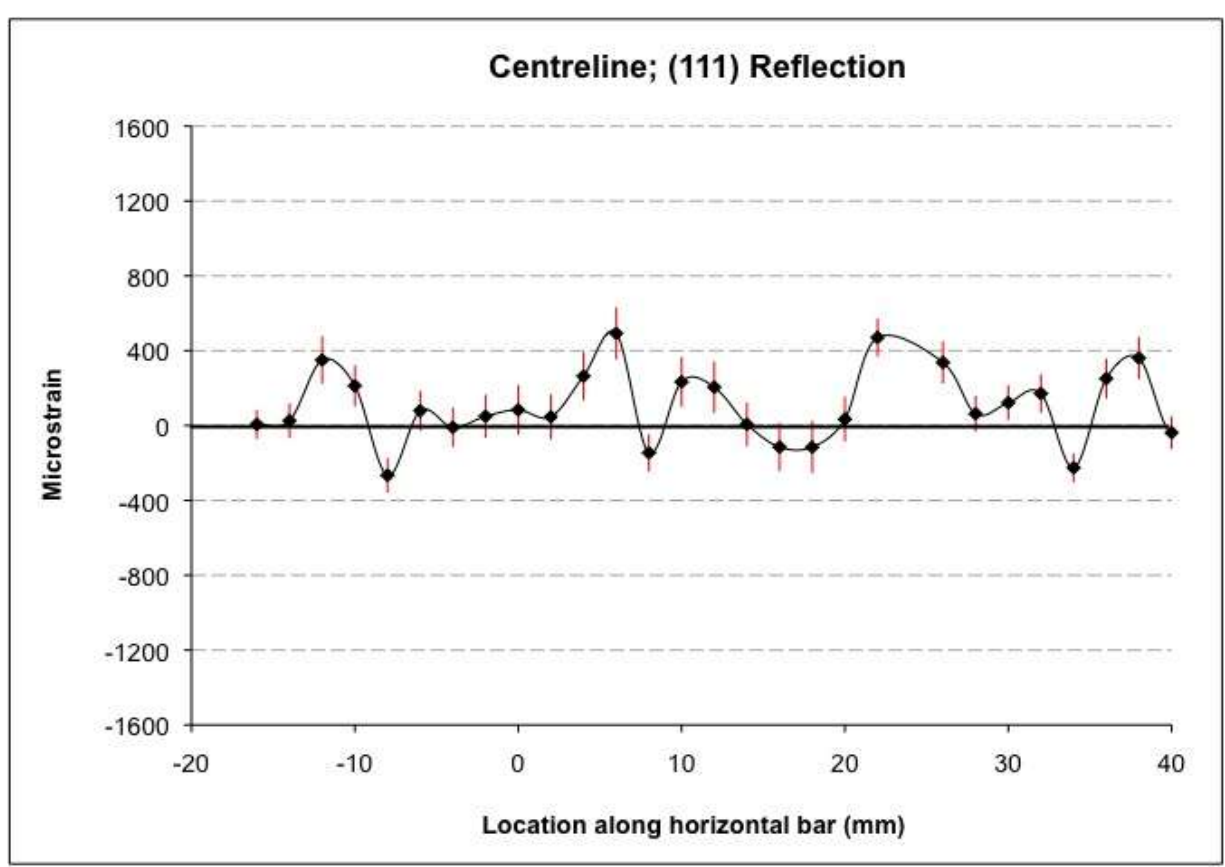

c)

Figure 4-36. Profiles of $\varepsilon_{x}$ at the sprue-bar junction along the a) top edge; b)bottom edge and c) centreline of the $0.02 \mathrm{wt} \%$ Ti alloy's horizontal bar for the (111) reflection. 


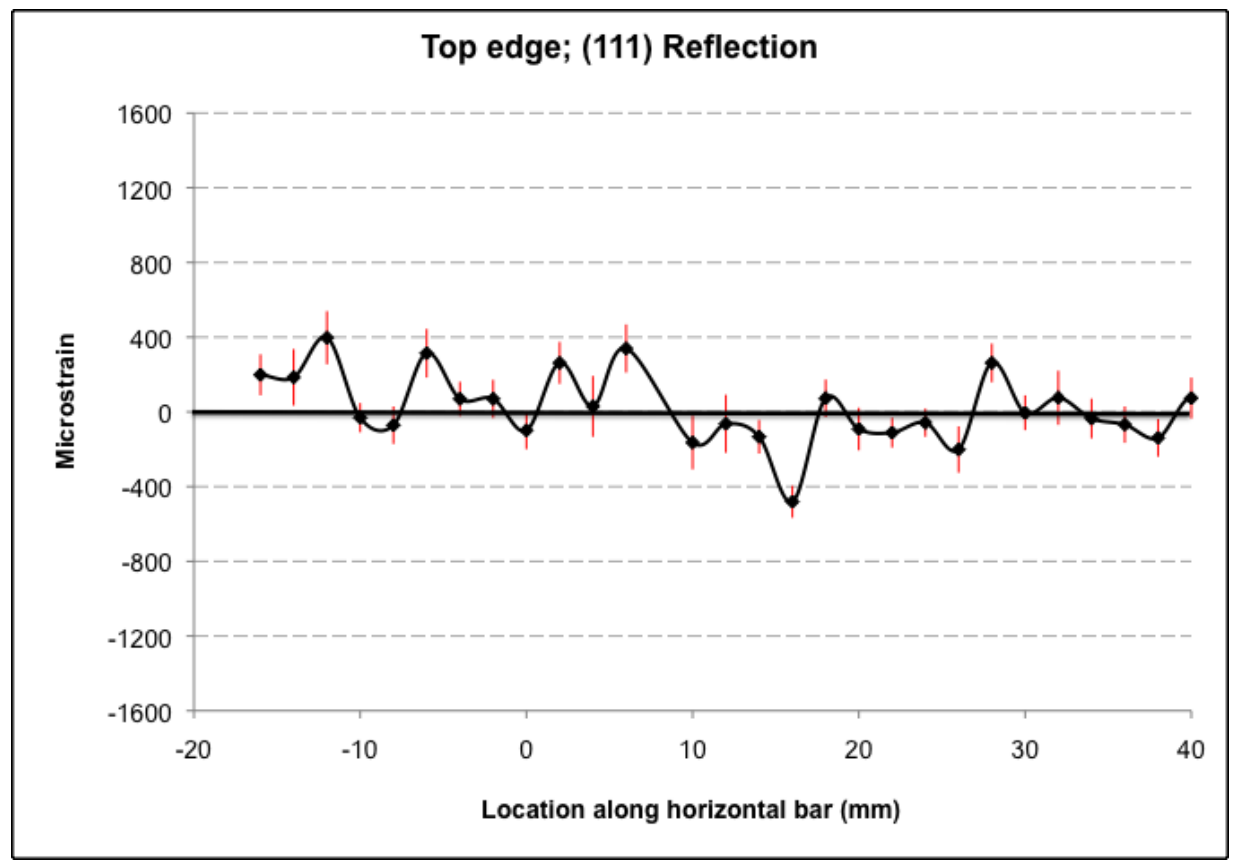

a)

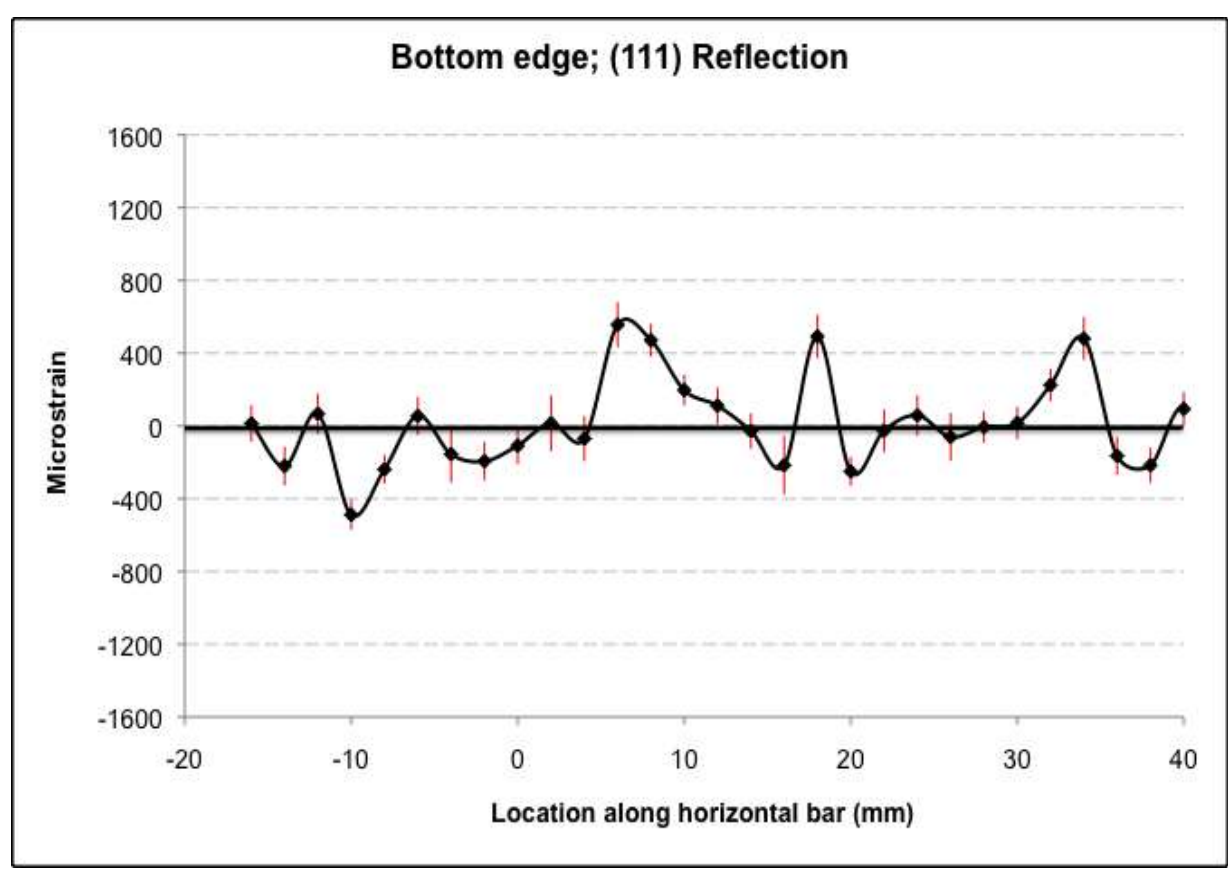

b) 


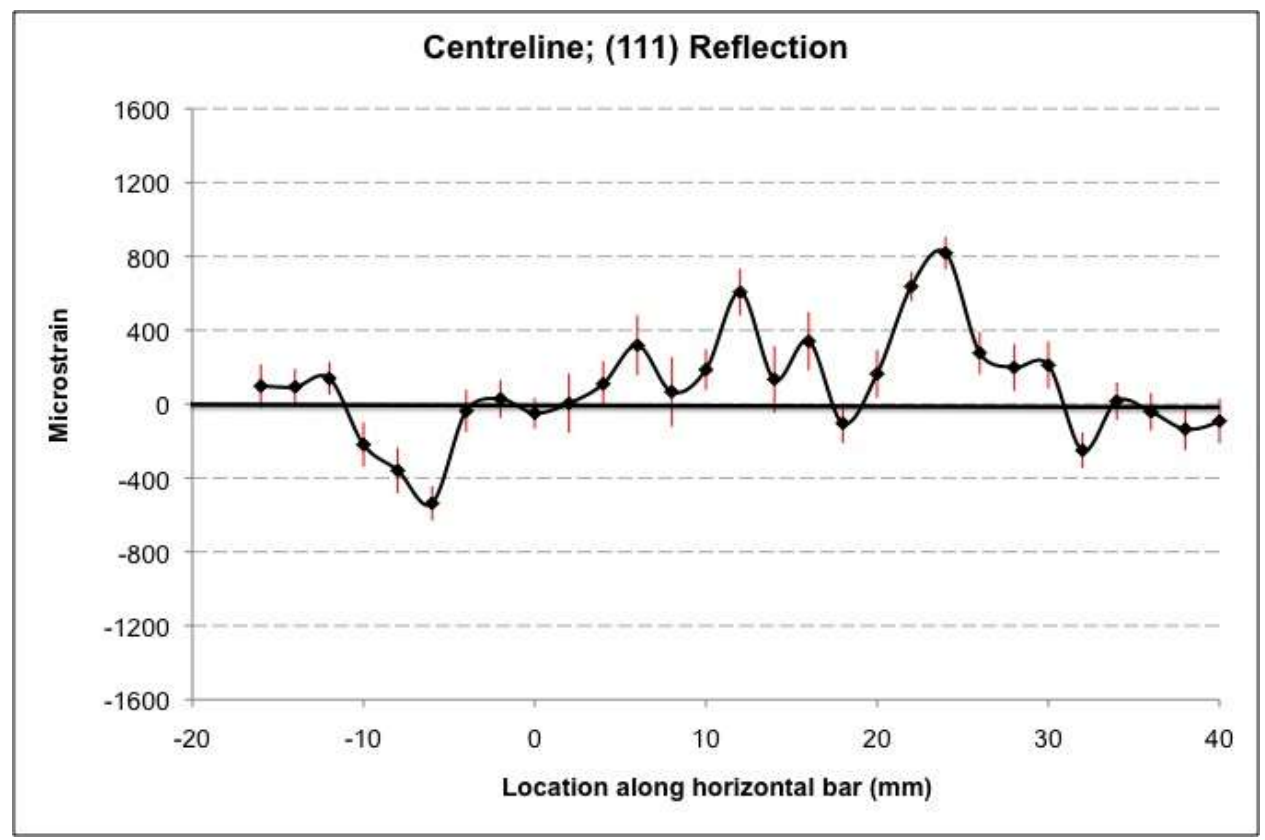

c)

Figure 4-37. Profiles of $\varepsilon_{x}$ at the sprue-bar junction along the a) top edge; b) bottom edge and c) centreline of the $0.05 \mathrm{wt} \%$ Ti alloy's horizontal bar for the (111) reflection.

The residual strain profiles at the sprue-bar junction showed good correlation between microstructure and hot tearing severity for the three B206 casting conditions. Residual strain was also measured along the remainder of the horizontal bar for the three castings and the results are presented in the next section.

\subsubsection{Strain Along the Horizontal Bar}

The profiles of $\varepsilon_{x}$ across the remainder of the horizontal bar (i.e. $x>40 \mathrm{~mm}$ ) are given in Figures 4-38 to 4-40 for the three B206 casting conditions. The measurements of strain were carried out along the (311) reflection for all alloys. In the case of the unrefined alloy, the strain was also measured along the (111) reflection, and these profiles are shown in Appendix 4. Again, the abscissa in the plots represents the distance along the horizontal bar. Strain measurements were carried out $160 \mathrm{~mm}$ along the length of the horizontal bar (i.e. at $x=160 \mathrm{~mm}$ ).

The strain profiles along the remainder of the horizontal bar $(40<x<160 \mathrm{~mm}$ ) revealed compressive $\varepsilon_{x}$ along both the top and bottom edge for the two refined castings. In the case of the unrefined casting, however, initial compression (between $40<x<110 \mathrm{~mm}$ ) followed by a small 
region (between $115<x<135 \mathrm{~mm}$ ) of tensile strain was observed. This region of tensile strain corresponded to the location of the hot tear (recall Figure 4-1). This suggests that the tensile $\varepsilon_{x}$ was sufficient to form a hot tear for this casting condition.

Tensile strain was expected along the full length of the horizontal bar due its restricted contraction by the end restraint and $90^{\circ}$ corner at the sprue-bar junction. In addition, previous research on $\mathrm{Mg}$ alloys [61,78] found that (using the same permanent mold) $x$-direction contraction induced tensile strain in the horizontal bar. Such was not the case for the B206 alloy, however. One possibility was that the end restraint might not have been effective at hindering the free contraction of the horizontal bar during solidification. To test this hypothesis, the unrefined B206 alloy was cast with a steel block (20 x $20 \mathrm{~mm}$ cross section) inserted into the casting cavity of the mold in order to prevent the molten alloy from flowing into the end restraint. The resulting 'unrestrained' B206 casting in Figure 4-41 shows that despite the 'elimination' of the end restraint, a hot tear formed at the middle of the horizontal bar. Hence, this confirmed the ineffectiveness of the end restraint at restricting contraction of the horizontal bar and inducing tensile strain. One possibility was that the end restraint's mechanical strength was not sufficient to do so. The results of thermal analysis and microscopy help provide reasoning for the formation of the hot tear.

Thermal analysis confirmed the middle of the horizontal bar as the last region along the horizontal bar to solidify (i.e. hot spot region). Thus, this suggests that once molten metal filled the casting cavity, the sections of the horizontal bar adjacent to the hot spot region solidified first (due to faster solidification times) and contracted in opposite directions (towards the colder regions of the mold). The free (unrestrained) contraction of these adjacent regions generated compressive strain, as confirmed in Figures 4-38a and b (for $40<x<115 \mathrm{~mm}$ and $x>135 \mathrm{~mm}$ ). In contrast, tensile strain was generated along the hot spot region (at $115<x<135 \mathrm{~mm}$ ) as interlocking dendrite arms attempted to accommodate such shrinkage. Further, due to the coarse dendritic morphology of unrefined B206, the remaining liquid metal was limited to small interdendritic regions and could not readily alleviate the induced strain along the hot spot. As a result, a hot tear formed along this region of the horizontal bar. Such was not the case for the refined alloys, however. 


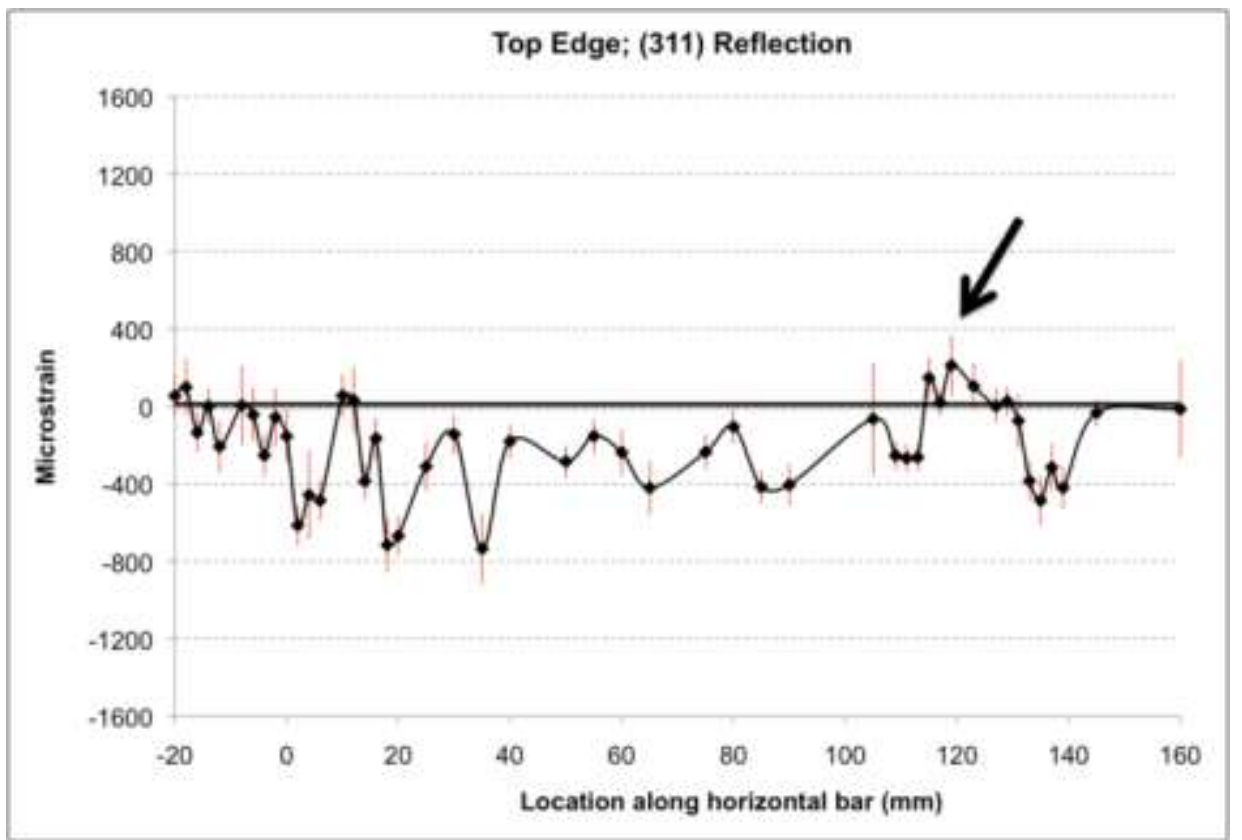

a)

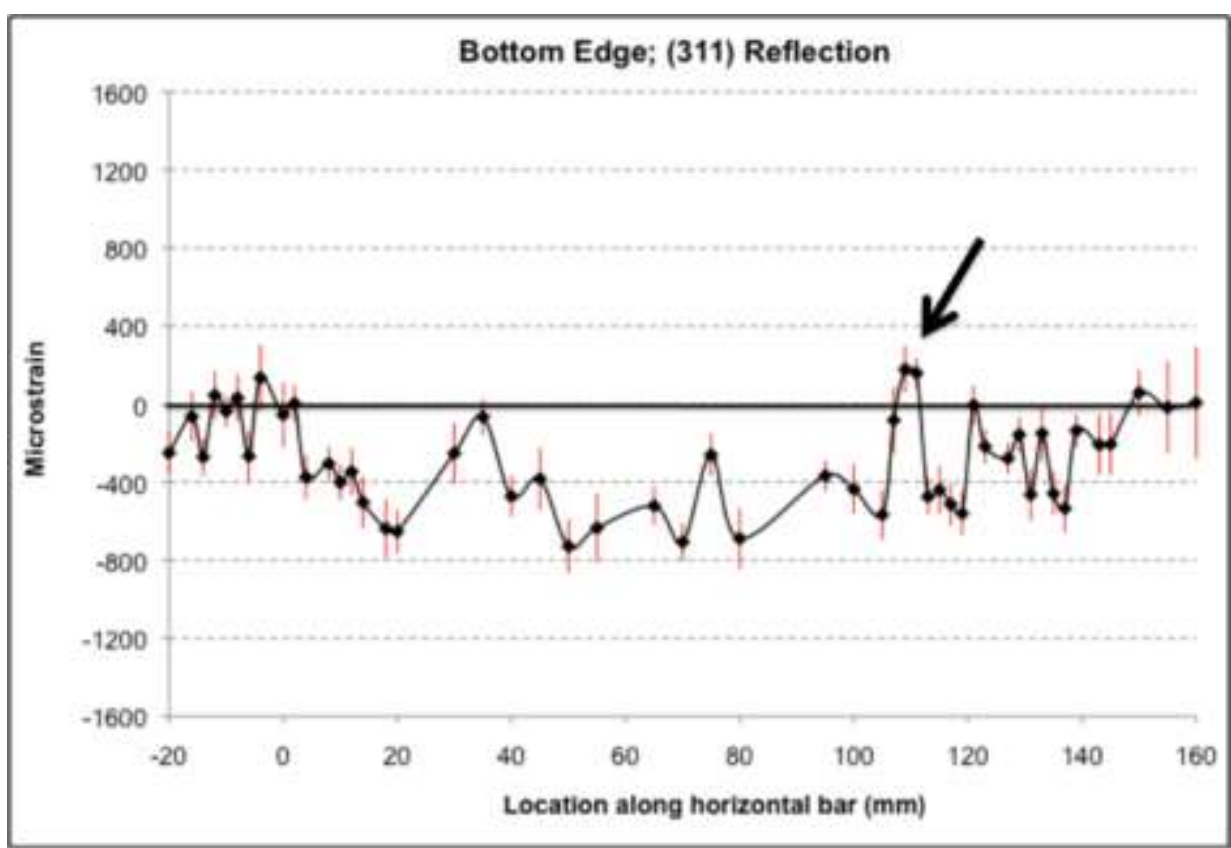

b)

Figure 4-38. Profiles of $\varepsilon_{x}$ along the a) top edge and b) bottom edge of the horizontal bar for the (311) reflection of the unrefined alloy. 


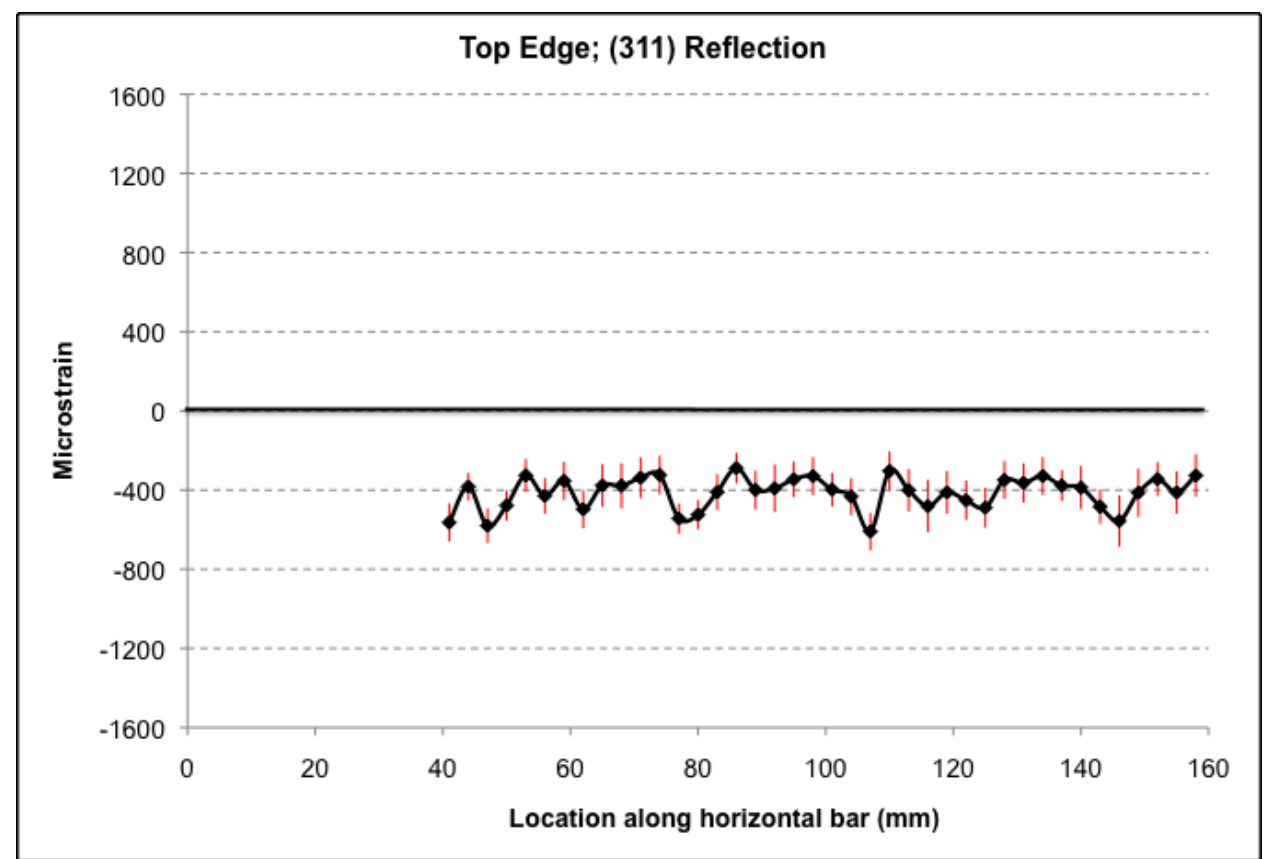

a)

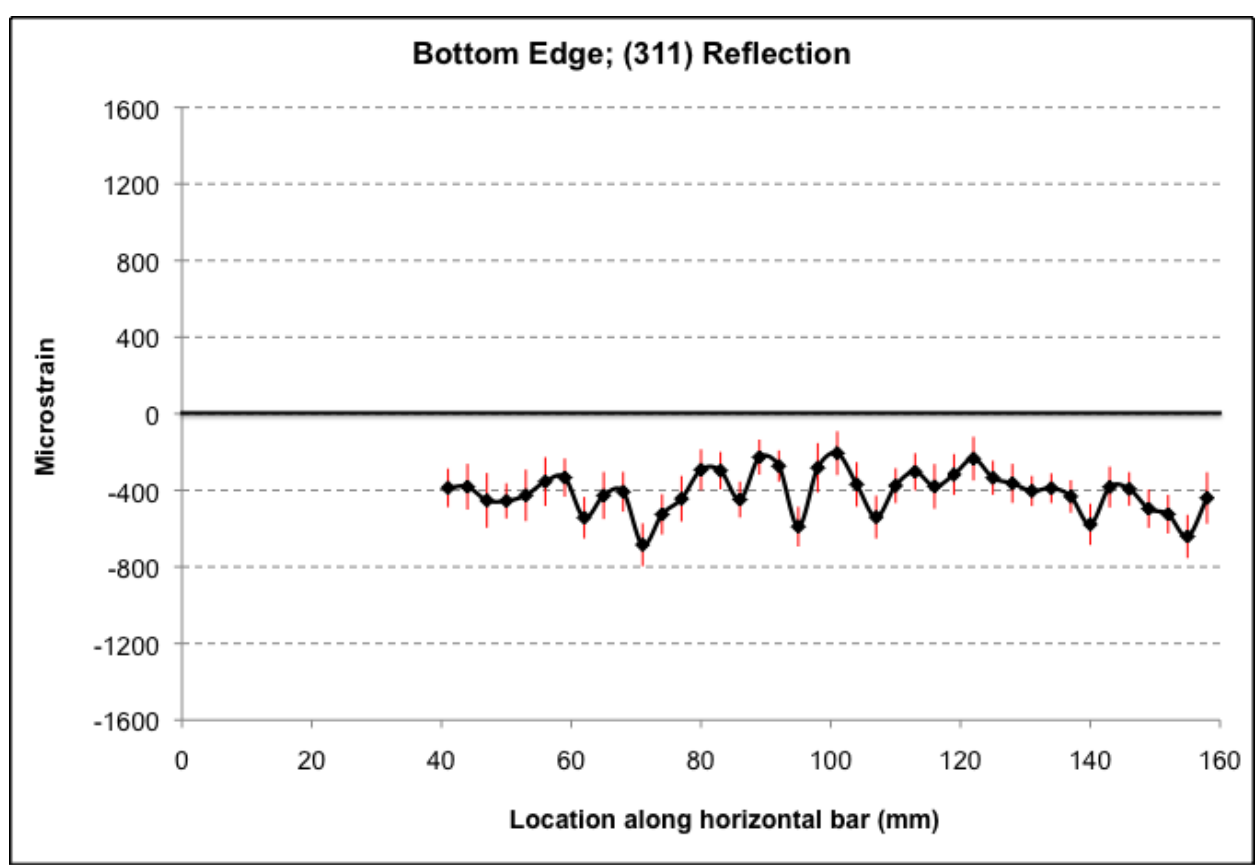

b)

Figure 4-39. Profiles of $\varepsilon_{x}$ along the a) top edge and b) bottom edge of the horizontal bar for the (311) reflection of the $0.02 \mathrm{wt} \% \mathrm{Ti}$ alloy. 


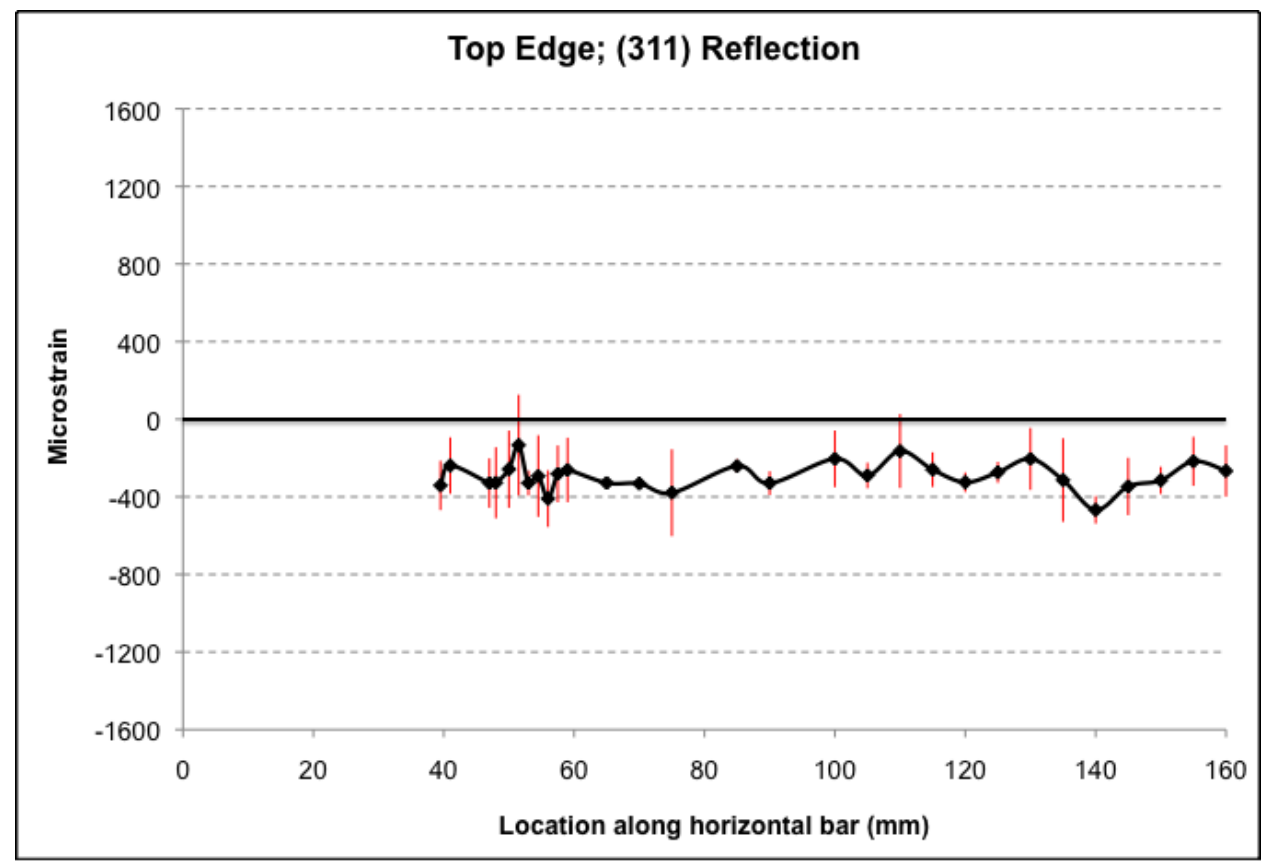

a)

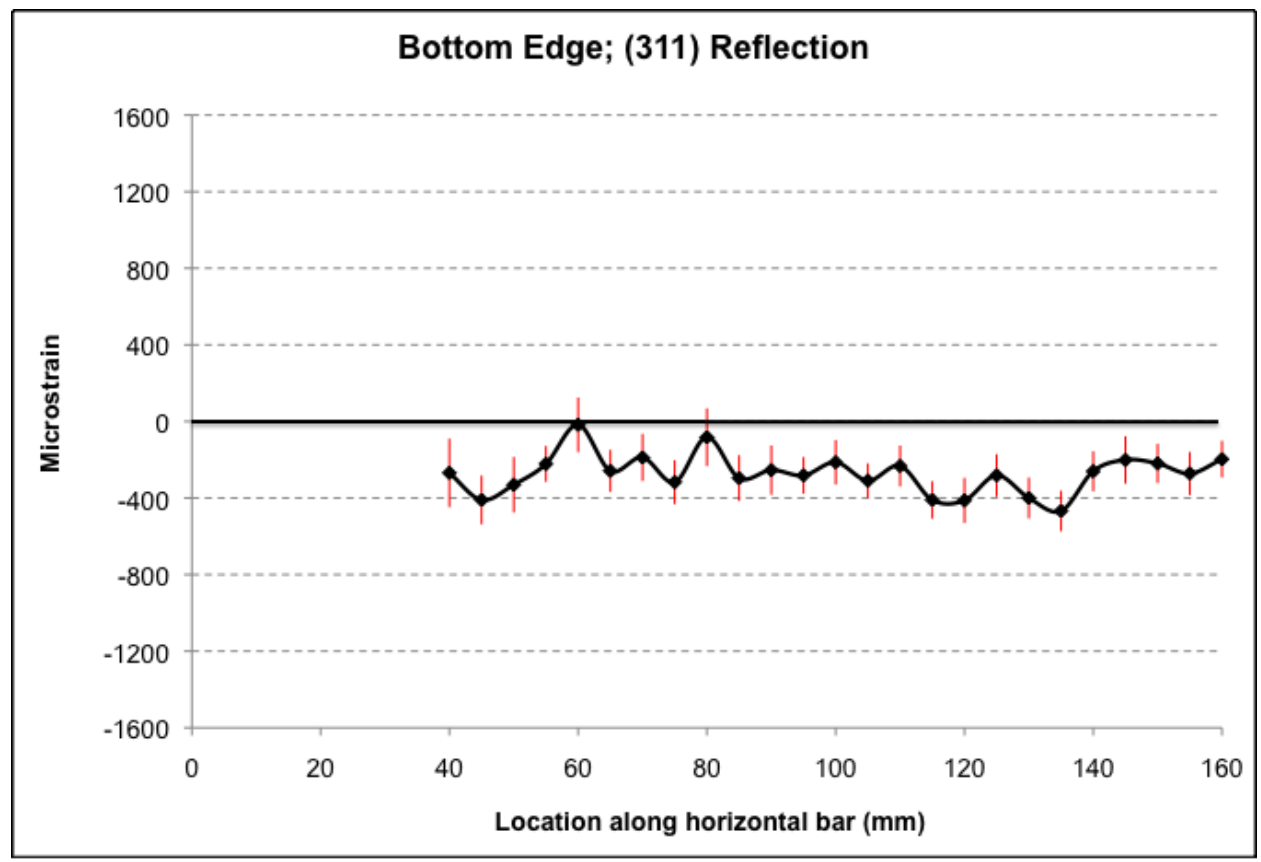

b)

Figure 4-40. Profiles of $\varepsilon_{x}$ along the a) top edge and b) bottom edge of the horizontal bar for the (311) reflection of the $0.05 \mathrm{wt} \% \mathrm{Ti}$ alloy. 


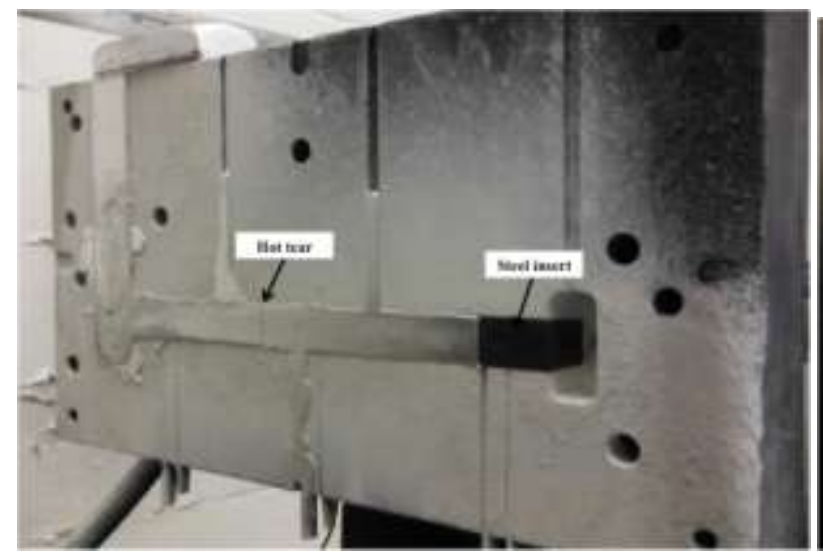

a)

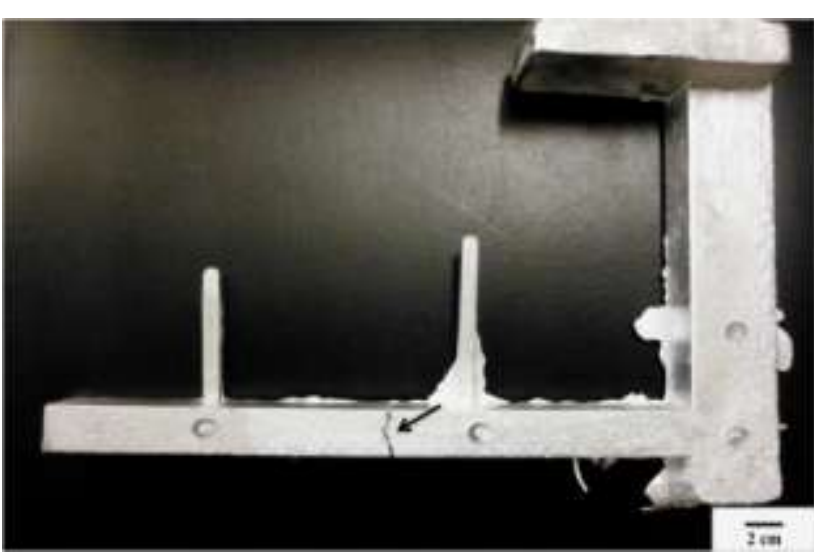

b)

Figure 4-41. Unrestrained B206 casting illustrating a) a front view of the hot tear (while inside the mold) and b) a rear view of the hot tear along the horizontal bar.

A more uniform distribution of compressive strain was observed along the top and bottom edge of the horizontal bar (Figures 4-39 and 4-40) for the refined casting conditions. Such uniform compression suggests that these castings were able to contract freely (i.e. without restriction) during solidification. As a result, hot tears did not initiate along the horizontal bar for either casting.

The variance in strain magnitude along the horizontal bar was calculated for the three casting conditions. The results in Figure 4-42 indicate that the distribution of strain was nearly twice as uniform along the refined castings as compared to that along the unrefined casting. These results are ascribed to the differences in alloy microstructure and solidification characteristics between the casting conditions. Thermal analysis confirmed the middle of the horizontal bar as the hot spot region in the refined castings as well. However, the strain at this region was effectively alleviated by the finer equiaxed grain morphologies of the refined castings. Specifically, in reference to the results of Bishop et al. [14] for a similar magnitude of strain, the strain concentrated at each of the more numerous small grain boundaries is less than the strain concentrated at each of the fewer boundaries of the large grains. Further, as suggested by the more uniform distribution of $\mathrm{Al}_{2} \mathrm{Cu}$ (Figures 4-12 and 4-13), the finer grains in the refined castings likely enabled uniform feeding of liquid at late stages of solidification. Lastly, the time available for mass feeding was enhanced in the refined castings due to the delay in onset temperature and time of coherency. Thus, such factors were crucial to improving the overall 
distribution of compressive strain and thereby increasing the resistance to hot tearing for the refined castings.

The strain profiles along the horizontal bar showed good agreement to the solidification characteristics and microstructure of unrefined and refined B206. In contrast to the sprue-bar junction, strain along this region was not influenced by the contracting downsprue. Moreover, the end restraint proved ineffective at restricting contraction of the horizontal bar. As a result, the required "mechanical factors" necessary for hot tearing (e.g. $90^{\circ}$ corner, geometrical constraint) as described by Pellini [13], were minimal along the horizontal bar. Nevertheless, the "metal factors" (e.g. microstructure, grain size) of unrefined B206 were incapable of alleviating the induced strain from shrinkage and thus a hot tear inevitably occurred. In contrast, the microstructure of the refined B206 castings enabled enhanced liquid metal feeding and thereby improved $x$-direction strain distribution and the ability of the alloy to resist hot tear formation. Residual strain measurements were also carried out along the $y$ and $z$-directions and the results are discussed next.

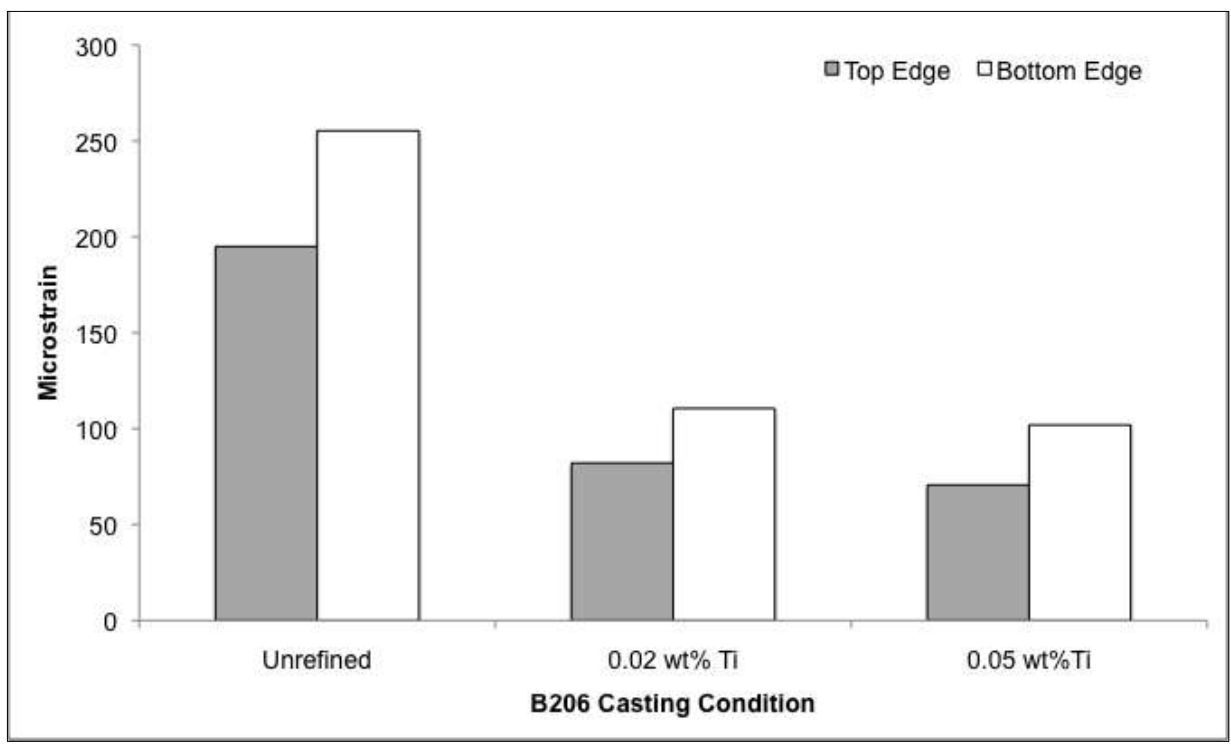

Figure 4-42. Variance in $x$-direction strain magnitude along the horizontal bar for the three B206 castings. 


\subsubsection{Residual Strain in the $y$-Direction}

Residual strain measurement in the $y$-direction $\left(\varepsilon_{y}\right)$ was carried out for the three B206 castings. Strain was measured along the length of the downsprue. This scan provided a depiction of the downsprue's axial deformation. Further, $\varepsilon_{y}$ was also measured along the top and bottom edge of the horizontal bar, which later enabled computation of residual stress along these linescans.

The results of $\varepsilon_{y}$ strain measurements along the top and bottom edge are presented in Figures 4-43 to 4-45. The strain along these regions was measured solely for the (311) crystallographic reflection. The abscissa in the plots represents the distance along the horizontal bar. Inspection of the plots reveals regions of high tension for the unrefined alloy. Specifically, the tensile $\varepsilon_{y}$ was observed at the sprue-bar junction and at the middle of the horizontal bar (i.e. location of the hot tear). In contrast, the profiles of $\varepsilon_{y}$ for the refined alloys demonstrate uniform compressive strain along the horizontal bar for both alloys.

The uniform compressive strain in the refined castings suggests that the contraction of the horizontal bar was unrestricted in the $y$-direction. The free contraction was expected, since there were no geometrical constraints imposed by the mold on the casting. As a result, strain along these directions did not affect the hot tearing tendency of the refined B206 castings. Nevertheless, despite the absence of such constraints, tensile $\varepsilon_{y}$ was observed for the unrefined casting. This was similar to the occurrence of tensile $\varepsilon_{x}$ along the horizontal bar despite the inability of the end restraint to restrict contraction (Figure 4-41). This suggests that the coarse dendrites in unrefined B206 were also not capable of accommodating casting contraction in the $y$-direction, particularly at critical regions (i.e. $90^{\circ}$ corner and hot spot). Thus, a high discontinuity in strain resulted along the unrefined casting. The discontinuity in strain is confirmed by the measurements of strain variance in Figure 4-46, which illustrate significant differences between the unrefined and refined castings. Consequently, it can be concluded that $\varepsilon_{y}$ contributed to the hot tearing tendency of unrefined B206. 


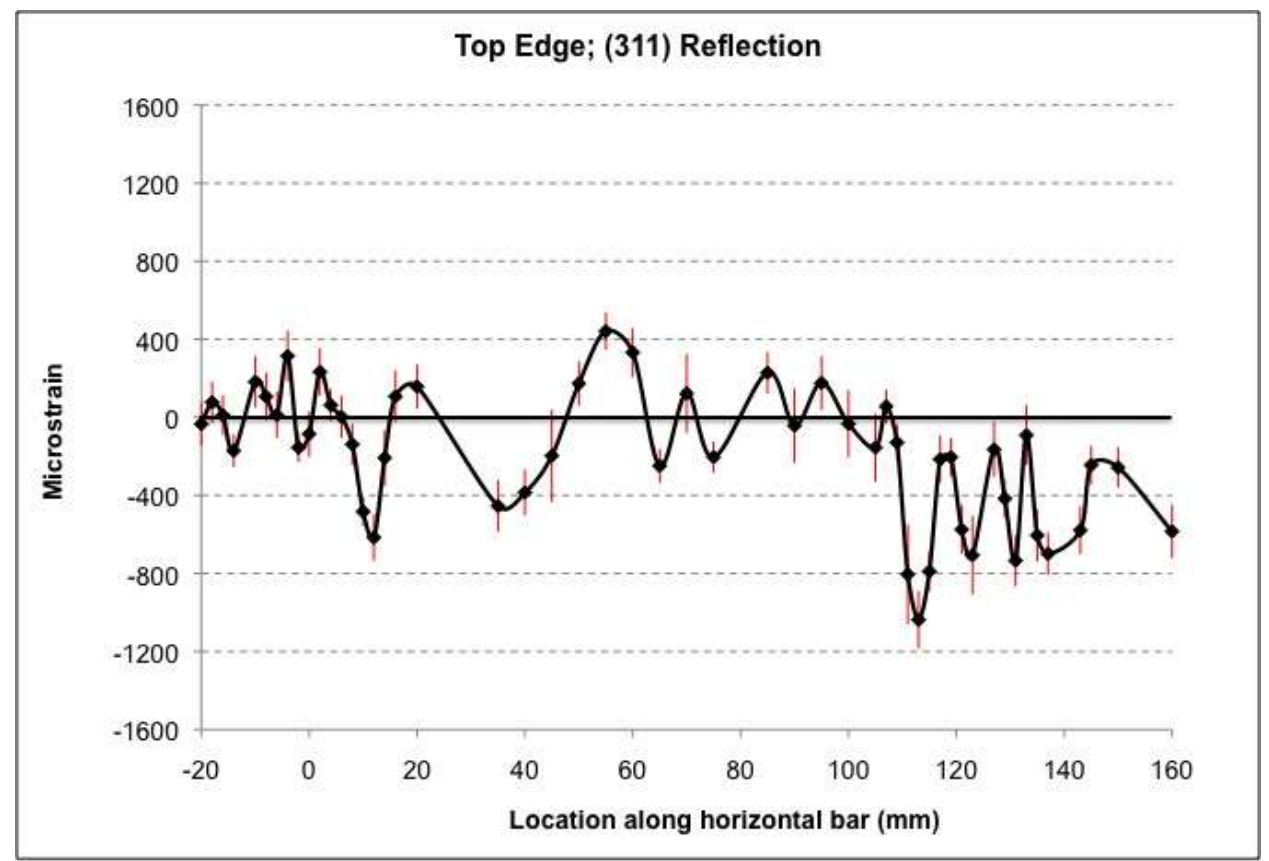

a)

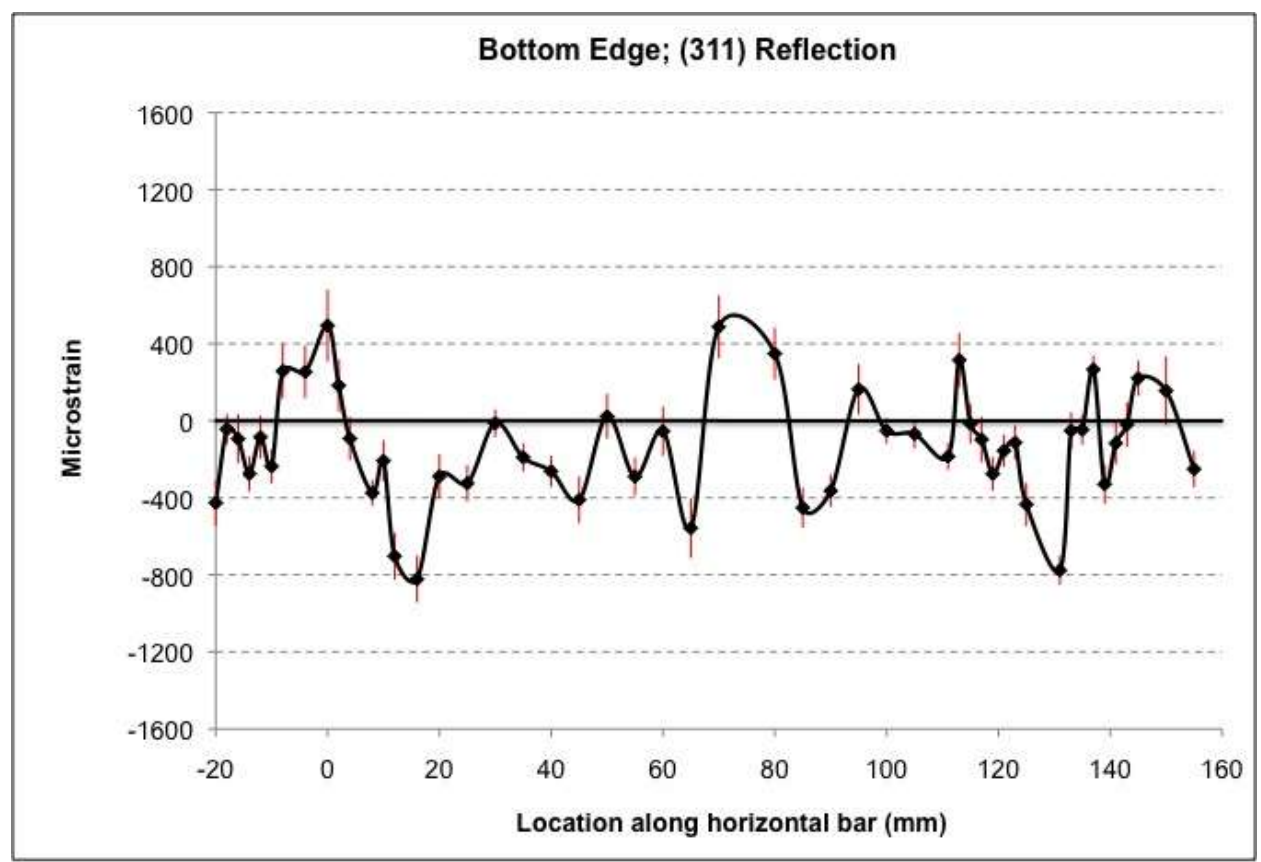

b)

Figure 4-43. Profiles of $\varepsilon_{y}$ along the a) top edge and b) bottom edge of the horizontal bar for the (311) reflection of the unrefined alloy. 


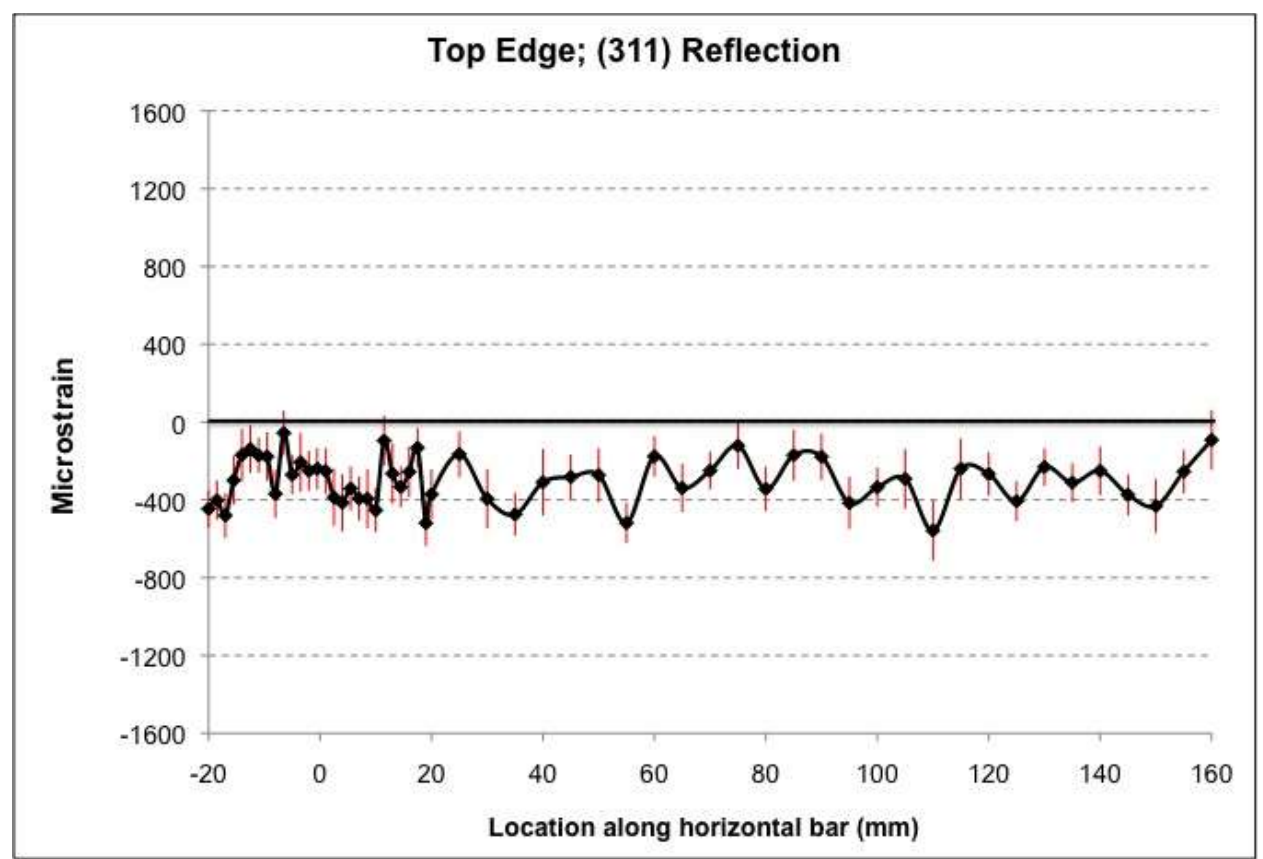

a)

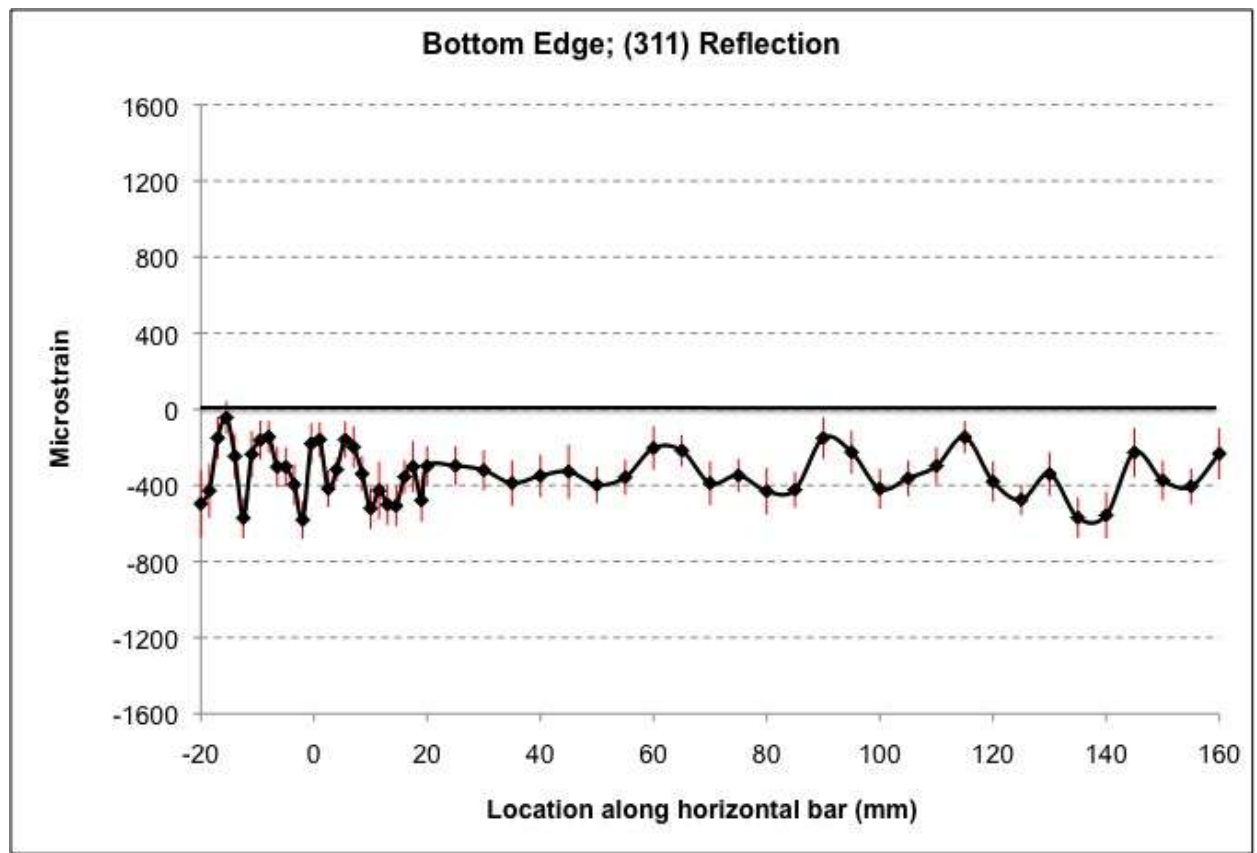

b)

Figure 4-44. Profiles of $\varepsilon_{y}$ along the a) top edge and b) bottom edge of the horizontal bar for the (311) reflection of the $0.02 \mathrm{wt} \% \mathrm{Ti}$ alloy. 


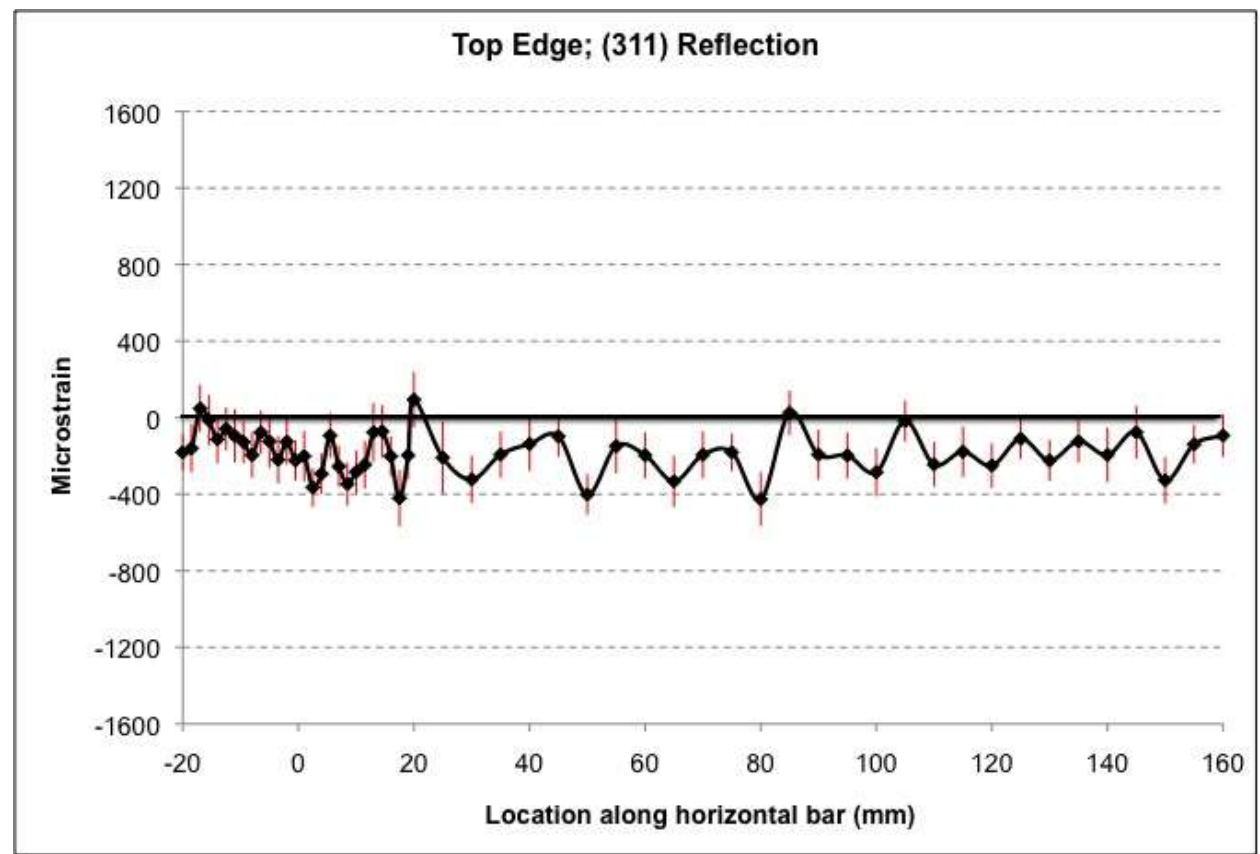

a)

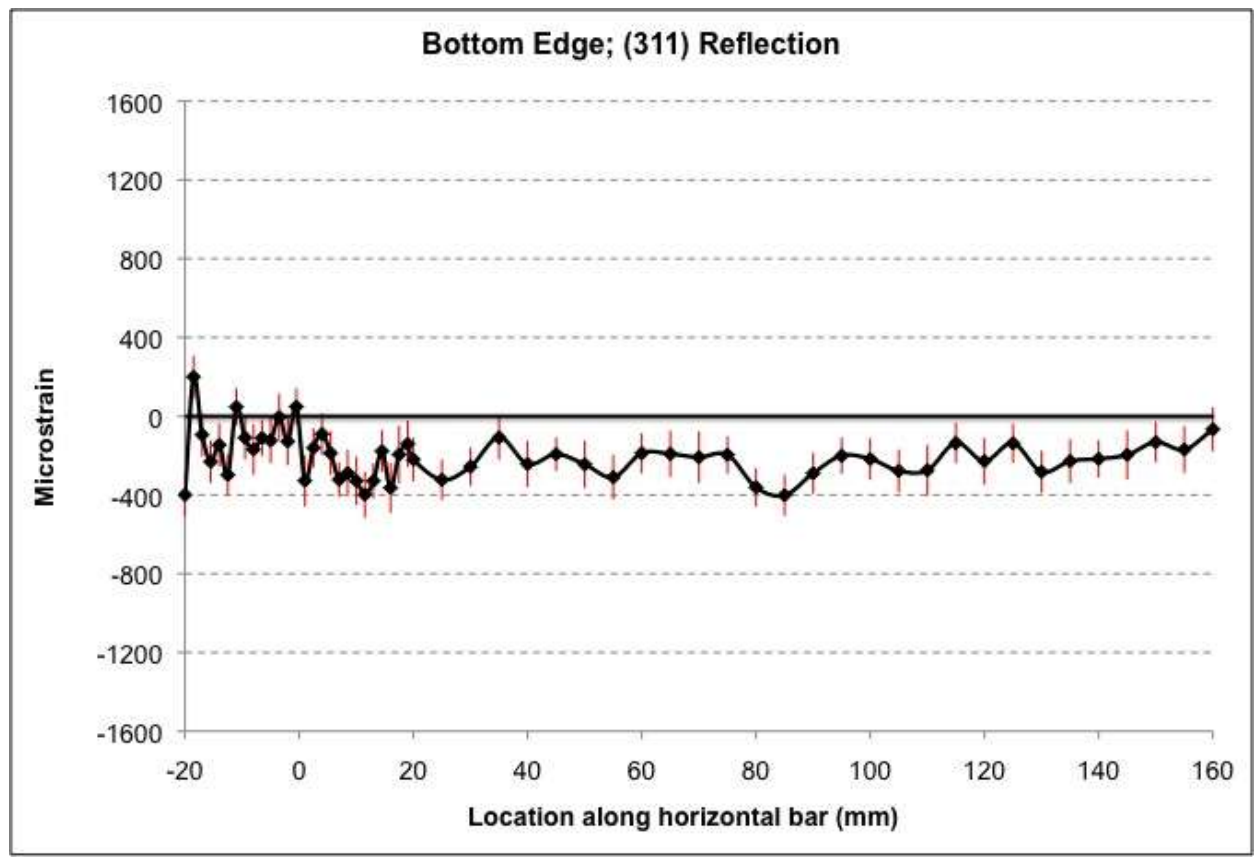

b)

Figure 4-45. Profiles of $\varepsilon_{y}$ along the (a) top edge and (b) bottom edge of the horizontal bar for the (311) reflection of the $0.05 \mathrm{wt} \% \mathrm{Ti}$ alloy. 


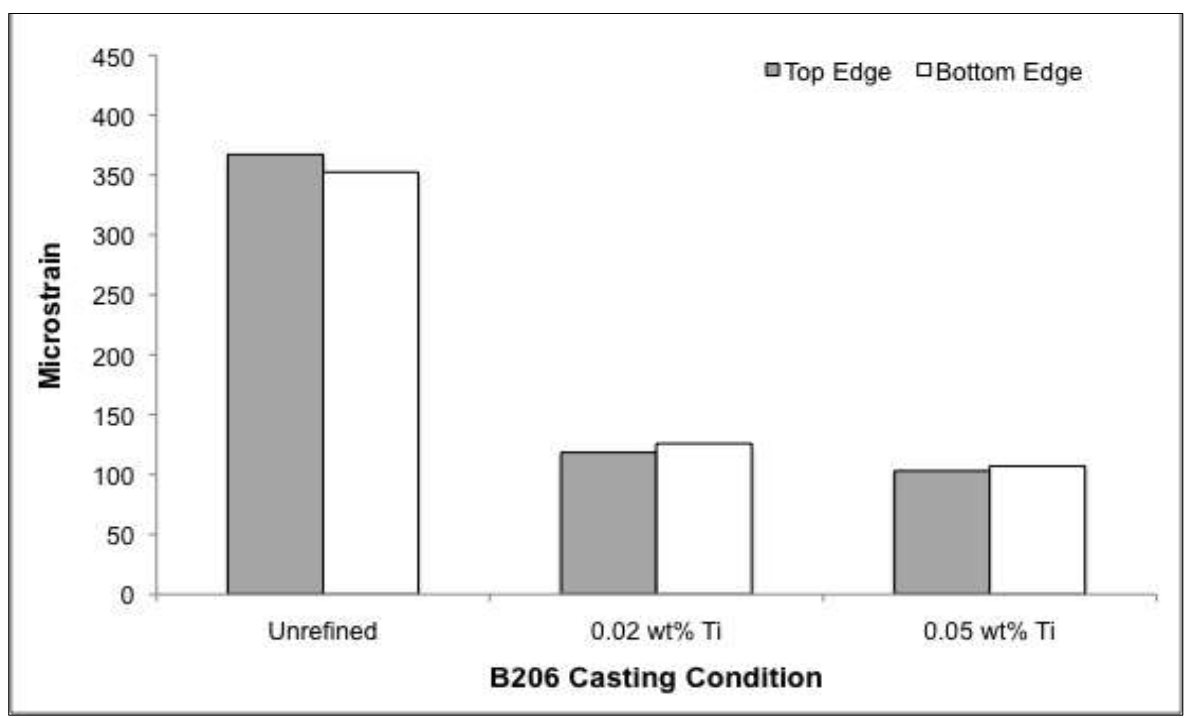

Figure 4-46. Variance in $y$-direction strain magnitude along the horizontal bar for the three B206 castings.

\subsubsection{Residual Strain Along the Downsprue}

The neutron scans along the downsprues are shown in Figure 4-47. Strain mapping was carried out only along the (111) reflection for the downsprue. Here, the abscissa in the plots represents the distance along the downsprue (i.e. $+y$-direction). The scans were carried out from the bottom of the downsprue towards the top (i.e. towards the pouring cup) and were started at approximately $10 \mathrm{~mm}$ below the bottom of the horizontal bar (Figure 3-11). The intersection of the downsprue with the bottom edge of the horizontal bar corresponds to a location of $y=0 \mathrm{~mm}$, while the intersection with the top edge corresponds to a location of $y=20 \mathrm{~mm}$.

The strain along the downsprue for the unrefined alloy is shown in Figure 4-47a. Initially, along the bottom of the downsprue $(-10<y<0 \mathrm{~mm})$, high compressive strain was observed. This suggests that this region underwent free (unrestrained) contraction. Upon reaching the junction with the horizontal bar $(y=0 \mathrm{~mm})$, however, the strain increased to a nearly tensile state. This increase in strain was likely attributed to the 'bending moment' that occurred at this region. The mutual influence of the horizontal bar and downsprue's contraction was therefore also visible in the scan along the downsprue. The remainder of the downsprue $(y>25 \mathrm{~mm})$ was in compression, thereby suggesting free contraction of the downsprue towards the pouring cup. 


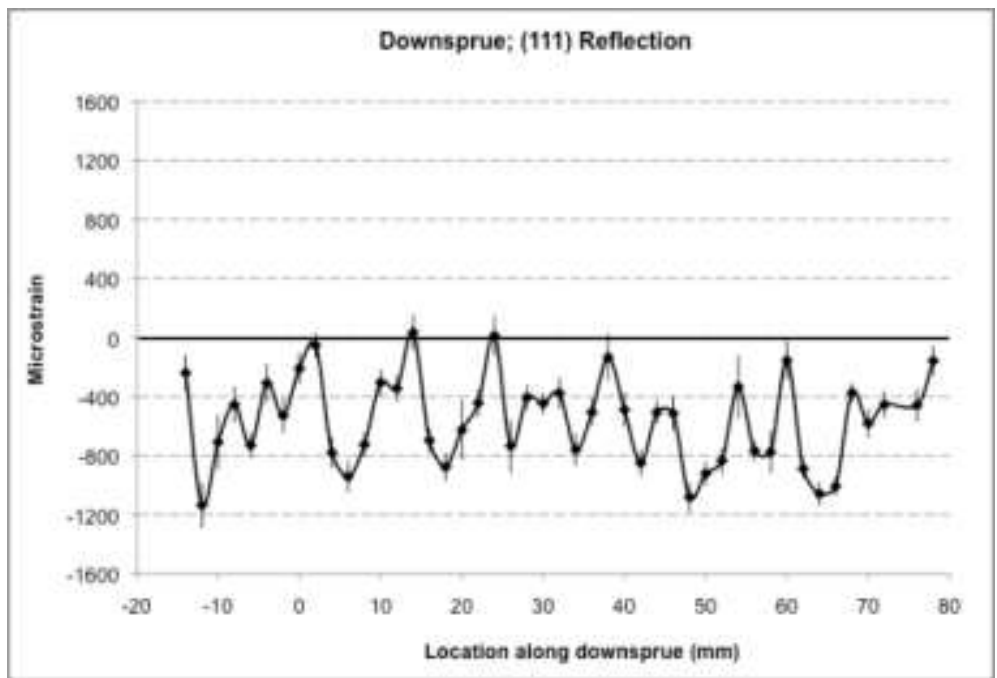

a)

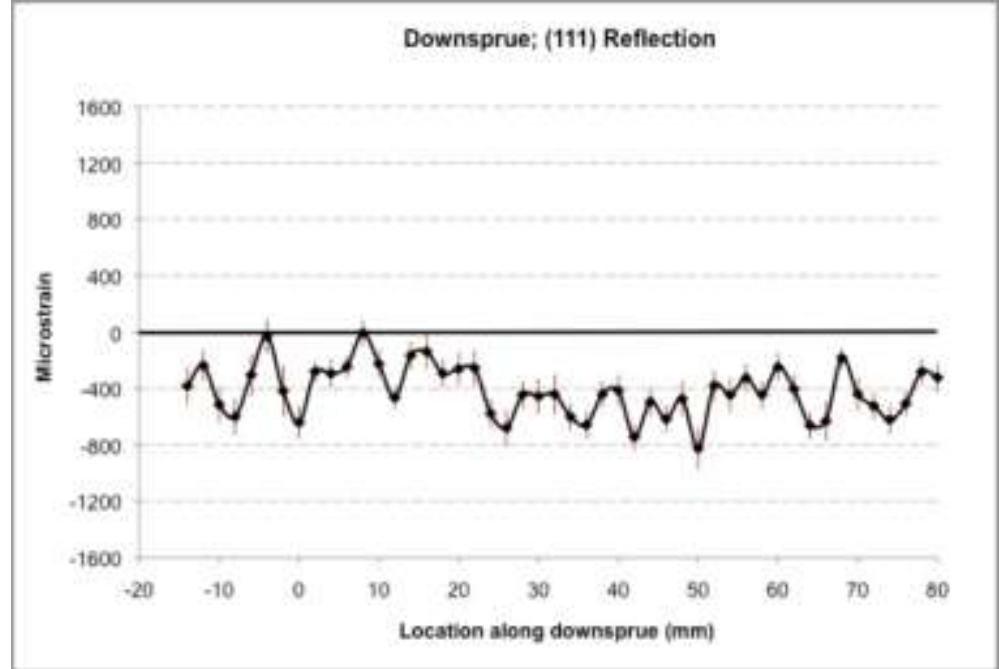

b)

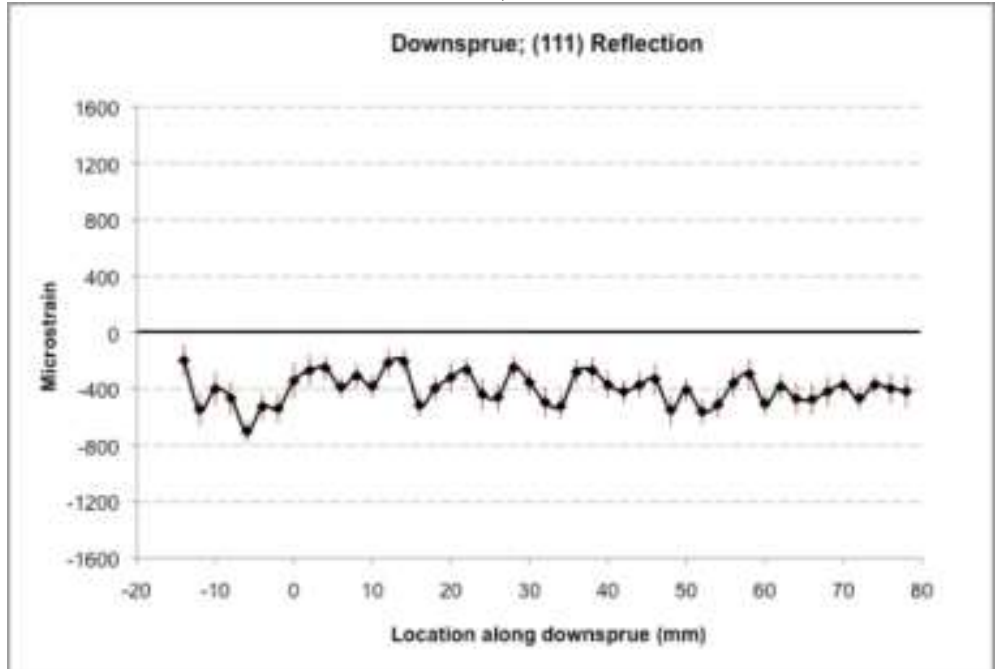

c)

Figure 4-47. Profiles of $\varepsilon_{y}$ along the downsprue of the a) unrefined B206; b) $0.02 \mathrm{wt} \%$ Ti and c) $0.05 \mathrm{wt} \% \mathrm{Ti}$ alloys for the (111) reflection. 
The residual strain profiles along the downsprue of the refined alloys are shown in Figure 4-47b and c. A similar profile to the unrefined alloy was observed for the refined alloys as well. However, the discontinuity in relative strain magnitude between $0<y<20 \mathrm{~mm}$ (i.e. the intersection with the horizontal bar) was less pronounced for the refined alloys. Comparing Figures 4-47b and 4-47c suggests that the discontinuity at the intersection with the horizontal bar was greater for the $0.02 \mathrm{wt} \% \mathrm{Ti}$ alloy than for the $0.05 \mathrm{wt} \% \mathrm{Ti}$ alloy. This was in agreement to both the profiles of axial strain $\left(\varepsilon_{x}\right)$ along the horizontal bar and the resulting hot tearing severity of these alloys at this region. The strain profile along the downsprue of the $0.05 \mathrm{wt} \% \mathrm{Ti}$ alloy suggests that the strain was not significant enough to form a hot tear in this alloy. In contrast, the strain along this region of the $0.02 \mathrm{wt} \% \mathrm{Ti}$ alloy promoted the formation of a hot tear.

\subsubsection{Residual Strain in the $z$-Direction}

The residual strain in the $z$-direction $\left(\varepsilon_{z}\right)$ was measured along the top and bottom edge of the horizontal bar for the three B206 castings. The $\varepsilon_{z}$ measurements were performed to enable calculation of residual stress along critical regions in the B206 castings. As was the case for the $y$-direction scan along these regions, the measurements were carried out solely for the (311) crystallographic reflection.

The results of the $\varepsilon_{z}$ linescans are presented in Figures 4-48 to 4-50. The unrefined alloy was scanned up to a distance of $160 \mathrm{~mm}$ from the $90^{\circ}$ corner, while the refined alloys were scanned up to a distance of $65 \mathrm{~mm}$ from the $90^{\circ}$ corner. The scans were limited to $65 \mathrm{~mm}$ due to limitations in the length of travel of the $z$-direction motor for the sample stage. However, in the case of the unrefined alloy, it was desired to scan the bar beyond $65 \mathrm{~mm}$ so as to include the region where the hot tear formed. In doing so, it was necessary to slice the bar and carry out two separate scans along the two portions of the horizontal bar. As a result, a gap is observed (between $65<x<90 \mathrm{~mm}$ ) in the profile for the unrefined alloy. The scan was not continued from $x=65 \mathrm{~mm}$ to ensure that the strain relief which occurred as a result of the cut was not included in the profile. This was not carried out for the refined alloys, since there was no hot tear present along this region for these conditions. 


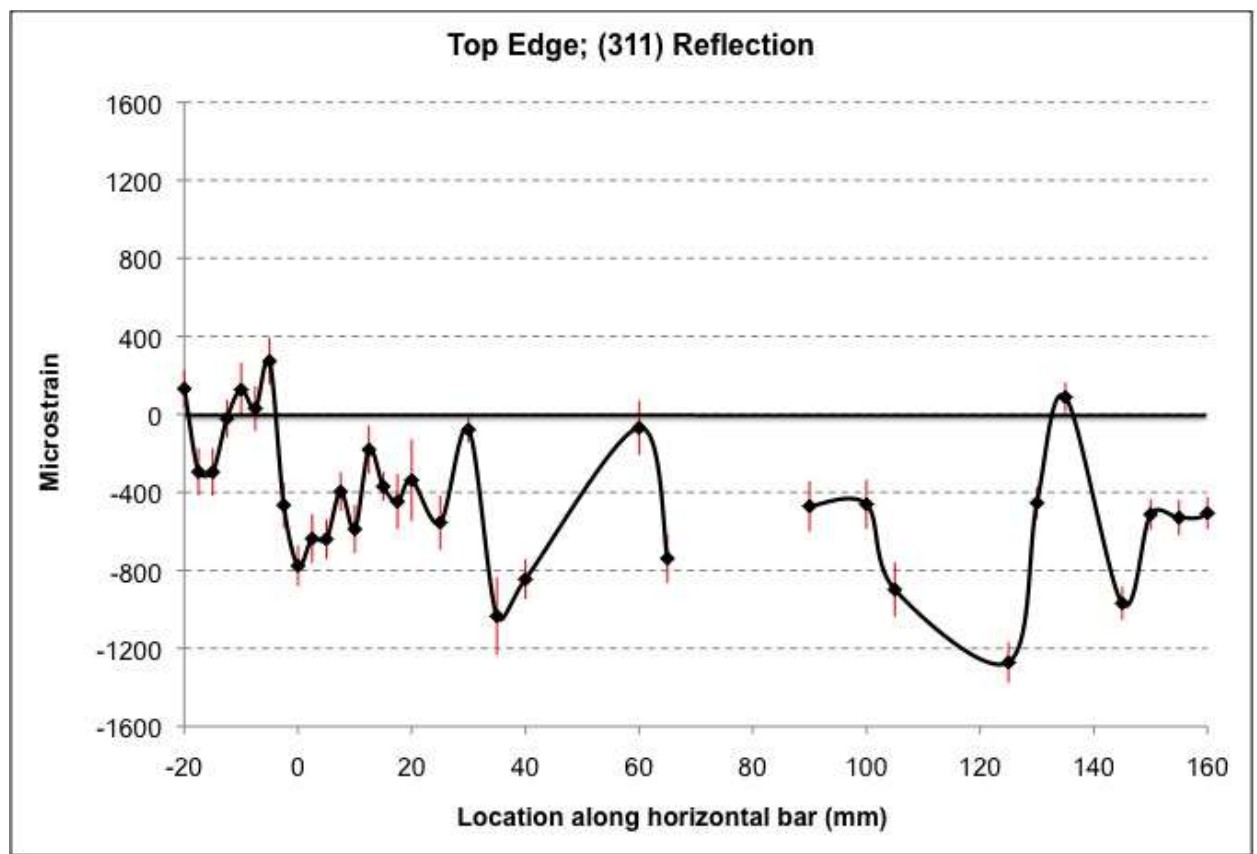

a)

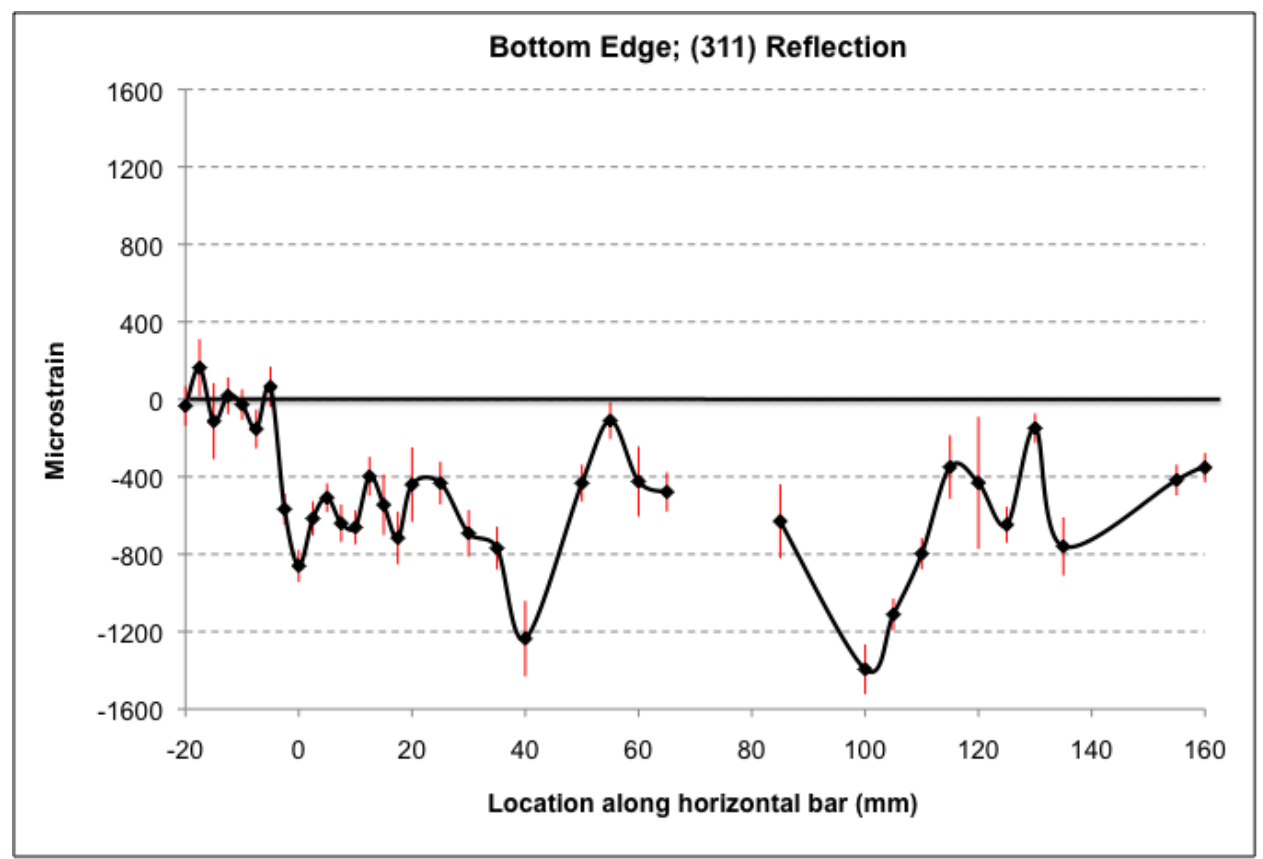

b)

Figure 4-48. Profiles of $\varepsilon_{z}$ along the a) top edge and b) bottom edge of the horizontal bar for the (311) reflection of the unrefined alloy. 


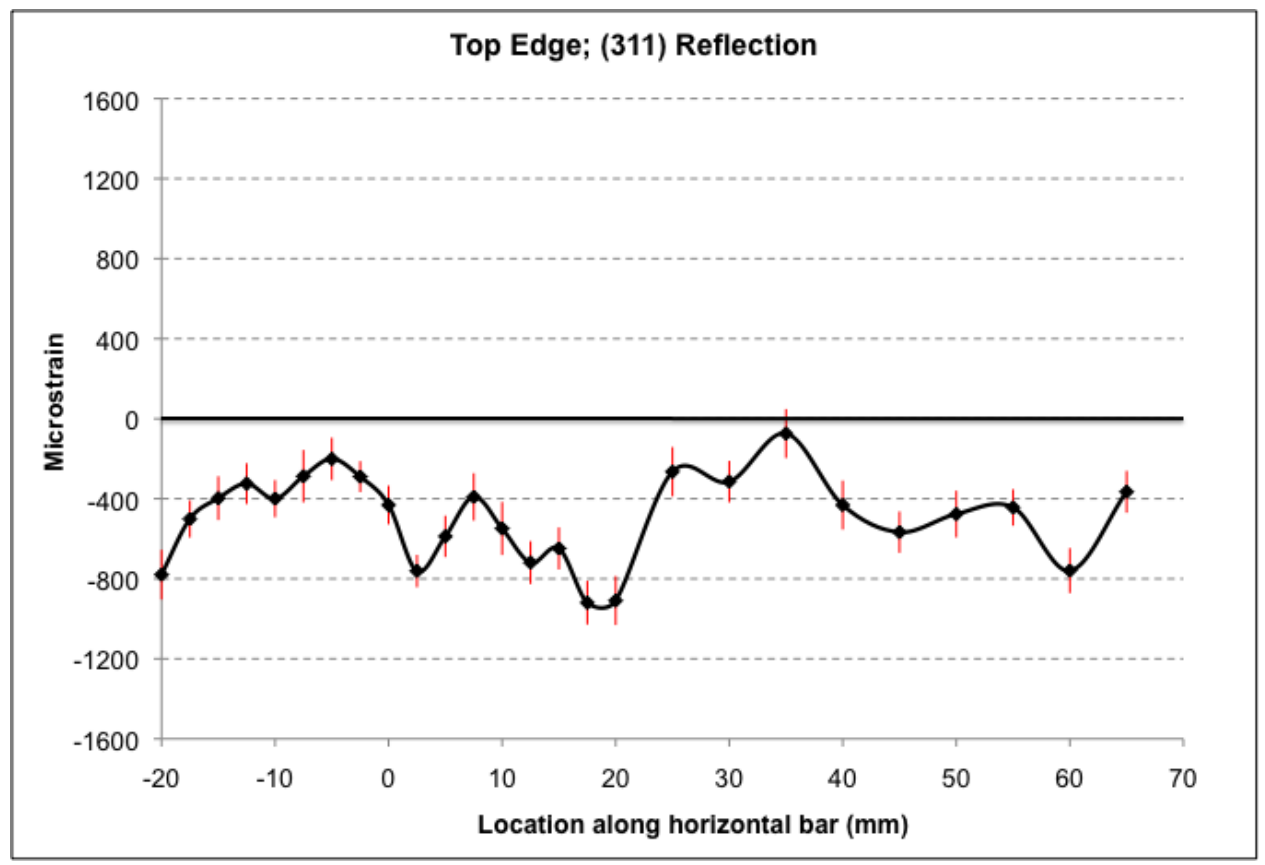

a)

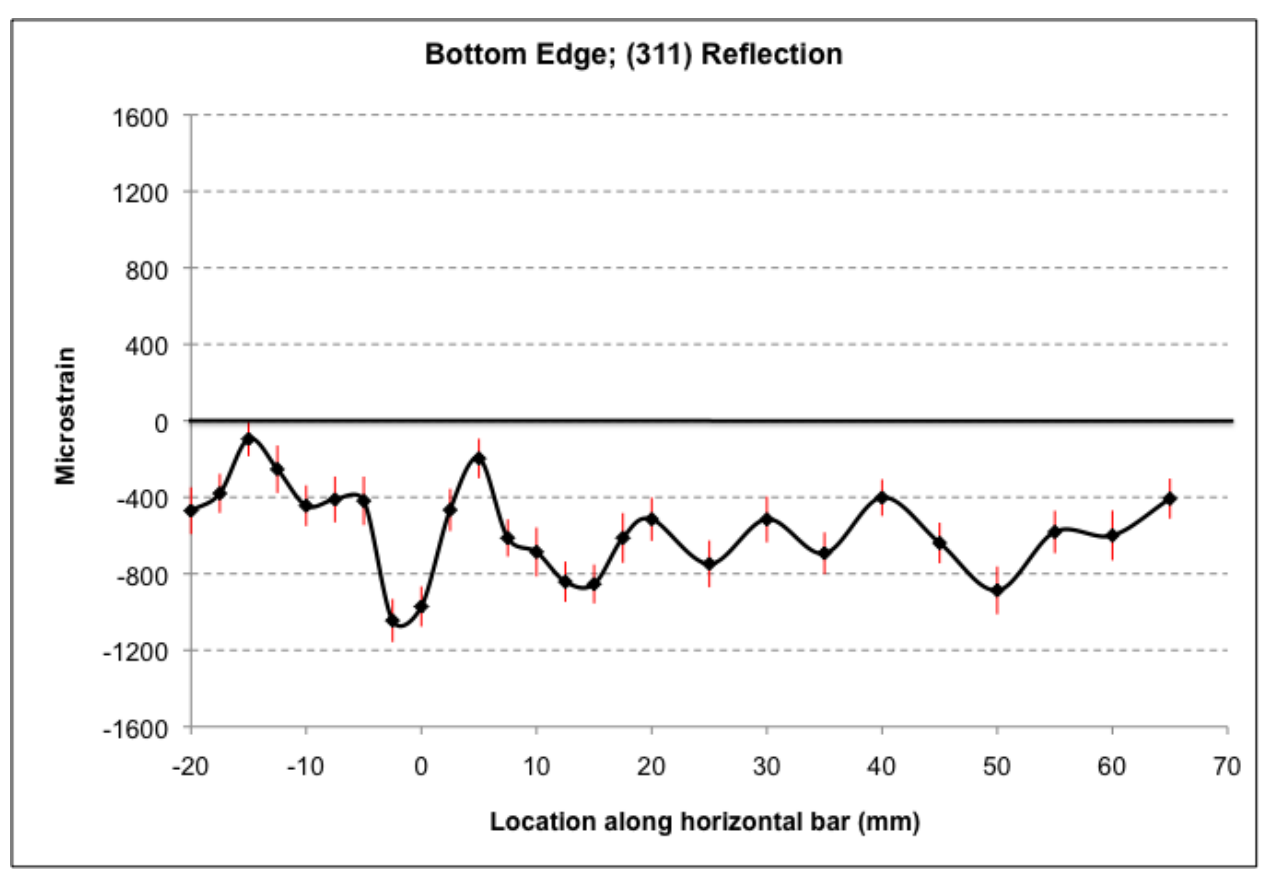

b)

Figure 4-49. Profiles of $\varepsilon_{z}$ along the a) top edge and b) bottom edge of the horizontal bar for the (311) reflection of the $0.02 \mathrm{wt} \% \mathrm{Ti}$ alloy. 


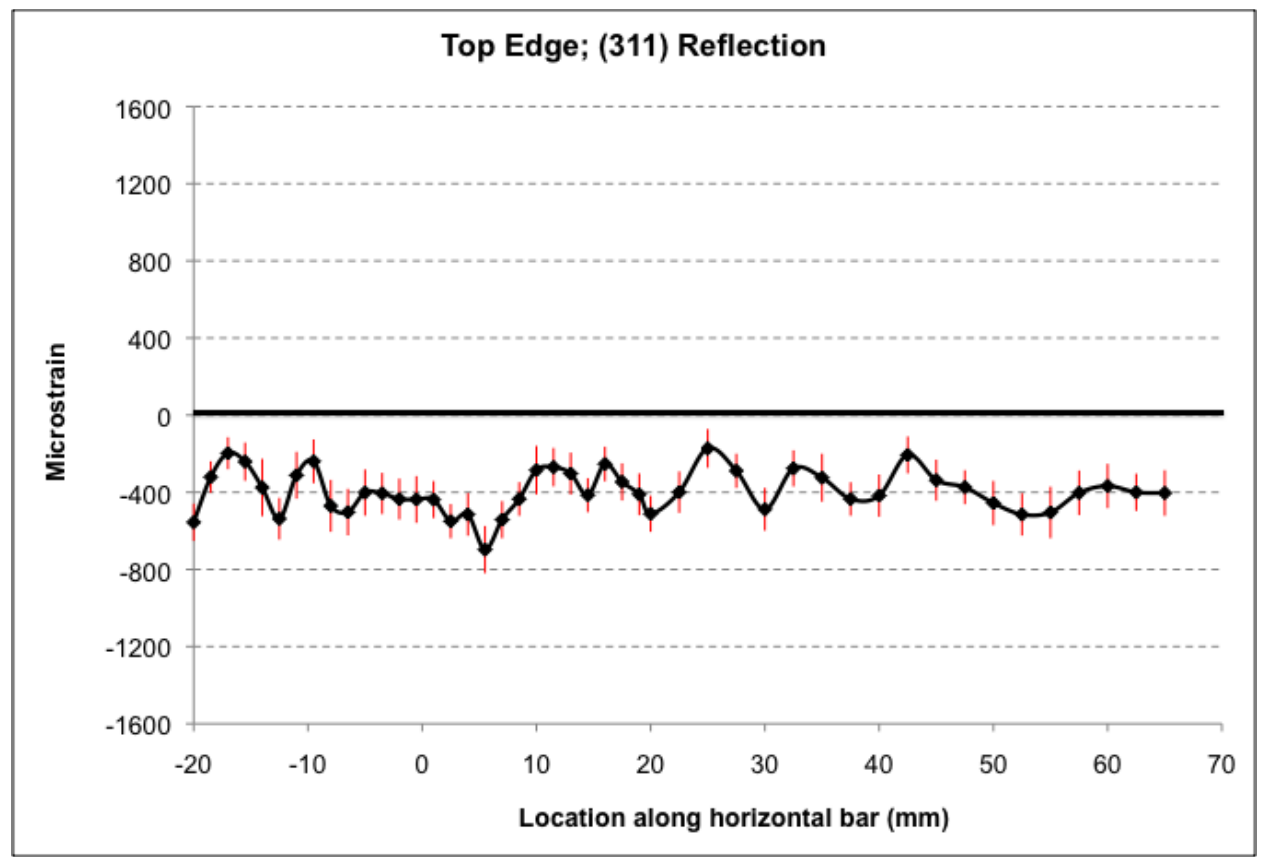

a)

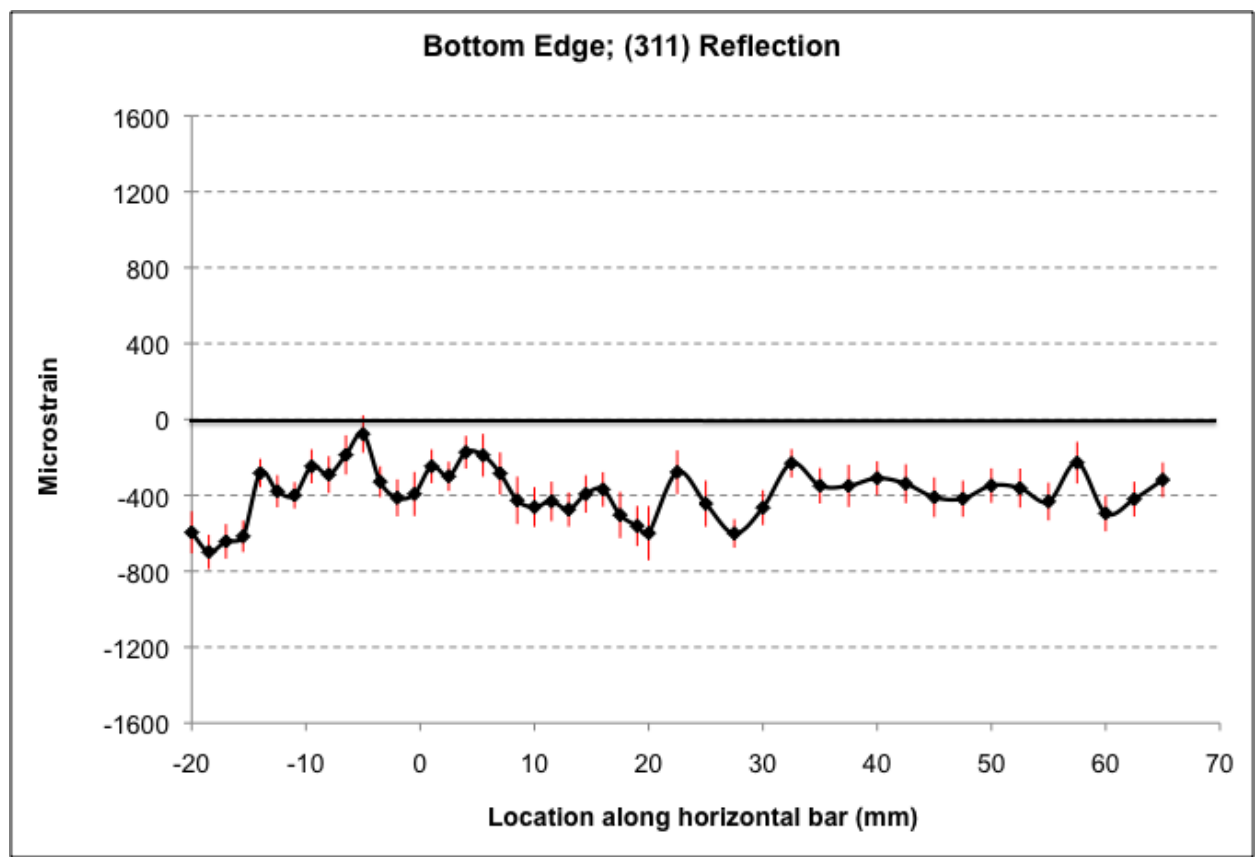

b)

Figure 4-50. Profiles of $\varepsilon_{z}$ along the a) top edge and b) bottom edge of the horizontal bar for the (311) reflection of the $0.05 \mathrm{wt} \% \mathrm{Ti}$ alloy.

As Figure 4-49 and 4-50 indicate, $\varepsilon_{z}$ remained compressive along the top and bottom edge for the refined alloys. A minor intensification in strain was observed at the sprue-bar junction (more so for the $0.02 \mathrm{wt} \% \mathrm{Ti}$ alloy) but the strain remained compressive. This was in agreement to the $\varepsilon_{y}$ 
profiles for these alloys and suggests that the refined castings were free (i.e. without restriction) to contract in the $z$-direction upon solidification. Similar to the $y$-direction, this was expected since there were no geometrical restrictions imposed by the mold on the castings. Hence, $\varepsilon_{z}$ likely did not contribute to the initiation and propagation of hot tears in the refined B206 castings.

In the case of the unrefined alloy, Figure 4-48 demonstrates that despite the absence of geometrical restrictions, tensile $\varepsilon_{z}$ was observed at both the sprue-bar junction and at the location of the hot tear (at $x \sim 135 \mathrm{~mm}$ ). This is further confirmation of the inability of the coarse dendrites in unrefined B206 to accommodate volumetric contraction of the casting. As the casting contracted in the $z$-direction, interlocking of large dendrite arms in the unrefined casting generated high tensile strain at critical regions. Such locations of tensile $\varepsilon_{z}$ resulted in nonuniform distribution of strain along the casting's horizontal bar, as depicted in Figure 4-51. In contrast, as the grain size reduced and the grain morphology transformed to a more globular structure, the variance in strain along the $z$-direction was found to reduce, thereby suggesting a more uniform distribution of $\varepsilon_{z}$. Thus, the strain variance along the $z$-direction further confirms the ability of the refined alloys to contract uniformly along the horizontal bar in this direction.

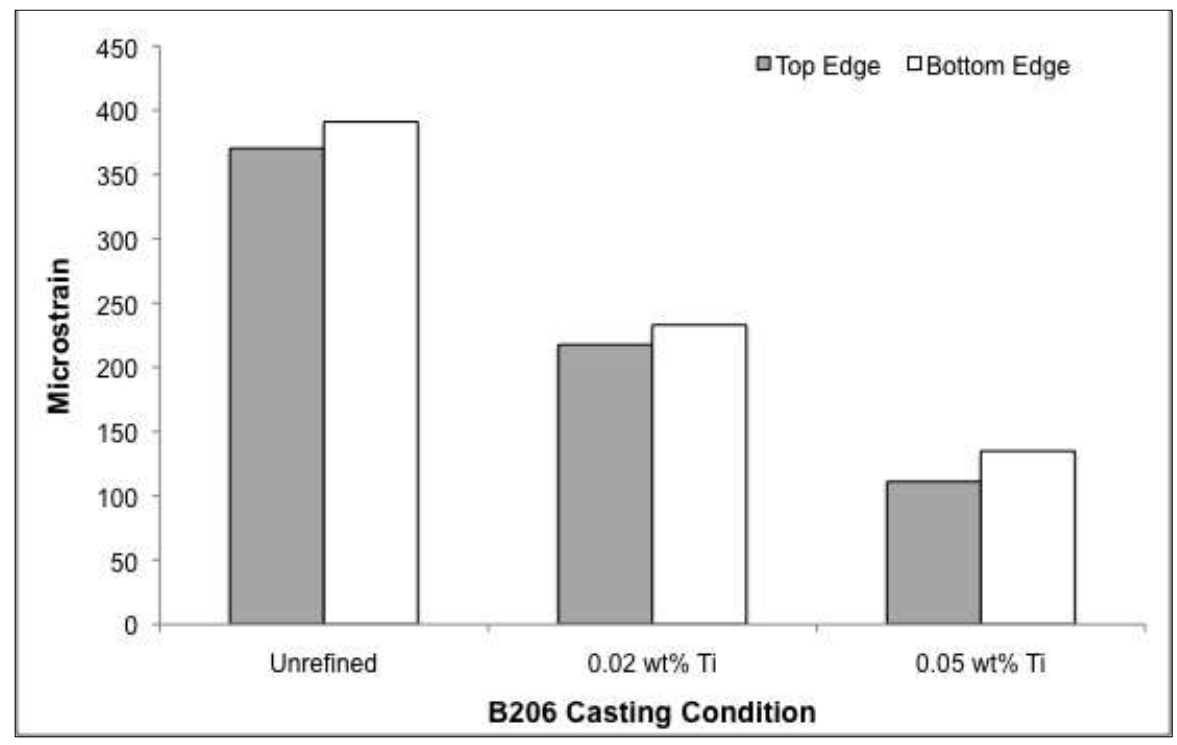

Figure 4-51. Variance in $z$-direction strain magnitude along the horizontal bar for the three B206 castings. 


\subsubsection{Residual Stress in B206 Castings}

The $\varepsilon_{x}, \varepsilon_{y}$ and $\varepsilon_{z}$ residual strains measured in the three B206 castings along the top and bottom edge of the horizontal bar were used in Equation 5 to calculate the principal stresses $\sigma_{x}, \sigma_{y}$ and $\sigma_{z}$. The elastic constants $E$ and $v$ were obtained from available literature [79]. The stress was calculated for the (311) crystallographic reflection for all directions. The bulk constants (i.e. $E$ and $v$ ) were deemed acceptable for stress calculations since only minor differences are observed in the degree of elastic anisotropy in Al (in particular for the (311) reflection) [67,80]. The profiles of residual stress are presented and discussed hereunder.

\subsubsection{Residual Stress in the $x$-Direction}

The strain measurements along the three B206 castings suggest that $\varepsilon_{x}, \varepsilon_{y}$ and $\varepsilon_{z}$ were mainly compressive. The residual stress calculated from these strains was expected to follow similar trends. The profiles of $\sigma_{x}$ are presented in Figures 4-52 and 4-53 for the horizontal bar top edge and bottom edge of the three B206 alloys. As was the case for the measurements of $\varepsilon_{z}$, a gap between $65<x<90 \mathrm{~mm}$ is observed in the profiles along the horizontal bar of the unrefined alloy. This enabled the $\sigma_{x}$ to be presented up to $160 \mathrm{~mm}$ along the horizontal bar from the $90^{\circ}$ corner (i.e. up to $x=160 \mathrm{~mm}$ ). In contrast, the stress was calculated up to a length of $65 \mathrm{~mm}$ from the $90^{\circ}$ corner (i.e. up to $x=65 \mathrm{~mm}$ ) along the horizontal bar for the refined alloys.

Inspection of the profiles demonstrate a good agreement with the profiles of $\varepsilon_{x}$, as compression was observed beyond the $90^{\circ}$ corner (i.e. $x=0 \mathrm{~mm}$ ) along the top edge, while a relative tension was seen along the bottom edge. In the case of the unrefined alloy (Figure 4-52), the tensile $\varepsilon_{x}, \varepsilon_{y}$ and $\varepsilon_{z}$ measured along the sprue-bar junction resulted in a mix of tensile and compressive $\sigma_{x}$ at this region. Beyond the sprue-bar junction, the $\sigma_{x}$ was then seen to significantly decrease further into compression along both the top and bottom edge before increasing again. Thus, a nonuniform distribution of $\sigma_{x}$ was observed along the horizontal bar of the unrefined alloy.

In contrast, the distribution of $\sigma_{x}$ was found to be more uniform along the horizontal bar of the refined castings (Figures 4-53a and b). This was in agreement to the strain profiles for these castings (Figures 4-39 and 4-40). Minor discontinuity in stress was observed at the sprue-bar 
junction. However, the tensile $\varepsilon_{x}$ measured along this region for the refined castings was exceeded by the compressive contributions of $\varepsilon_{\mathrm{y}}$ and $\varepsilon_{\mathrm{z}}$, thereby resulting in compressive $\sigma_{x}$. Along the bottom edge, a greater increase in compressive stress was observed along the profile of the $0.02 \mathrm{wt} \% \mathrm{Ti}$ alloy. This difference was likely attributed to the increased discontinuity observed in the strain profiles of the $0.02 \mathrm{wt} \%$ Ti alloy. Nevertheless despite such discontinuity, the stress remained compressive for the $0.02 \mathrm{wt} \% \mathrm{Ti}$ alloy. This suggests that tensile stress was not required to generate hot tears in B206, since only tensile $\varepsilon_{x}$ along the sprue-bar junction region was sufficient to form a hot tear along the 0.02 wt\% Ti alloy. Such results are in agreement with the arguments of Pellini [13] and Campbell [1] who claimed that strain (and strain rate) is more critical for hot tearing than stress.

\subsubsection{Residual Stress in the $y$ and z-Direction}

The profiles of $\sigma_{y}$ and $\sigma_{z}$ along the top and bottom edge of the horizontal bar are shown in Figures 4-54 to 4-56 for the three B206 alloys. Similar profiles of $\sigma_{y}$ and $\sigma_{z}$ were observed for each alloy. As was the case for $\sigma_{x}$, tensile stress along the $y$ and $z$-direction was calculated at the sprue-bar junction for the unrefined alloy. The remainder of the profile along the horizontal bar showed similar regions of discontinuity as observed in the $x$-direction.

In the case of the refined alloys, the distribution of stress across the horizontal bar was again more uniform and remained compressive. This was in agreement to the strain profiles for the refined alloys. An increase in compressive stress was again observed along the bottom edge for the $0.02 \mathrm{wt} \% \mathrm{Ti}$ alloy, in agreement to the profile of $\sigma_{x}$. In contrast, the stress at this region for the $0.05 \mathrm{wt} \% \mathrm{Ti}$ alloy was fairly uniform. Beyond the sprue-bar junction region, the remainder of the profile suggests that the stress remained uniformly compressive for both refined alloys. As a result, the stress in these directions was not significant in initiating and propagating hot tears in the refined B206 alloys. 


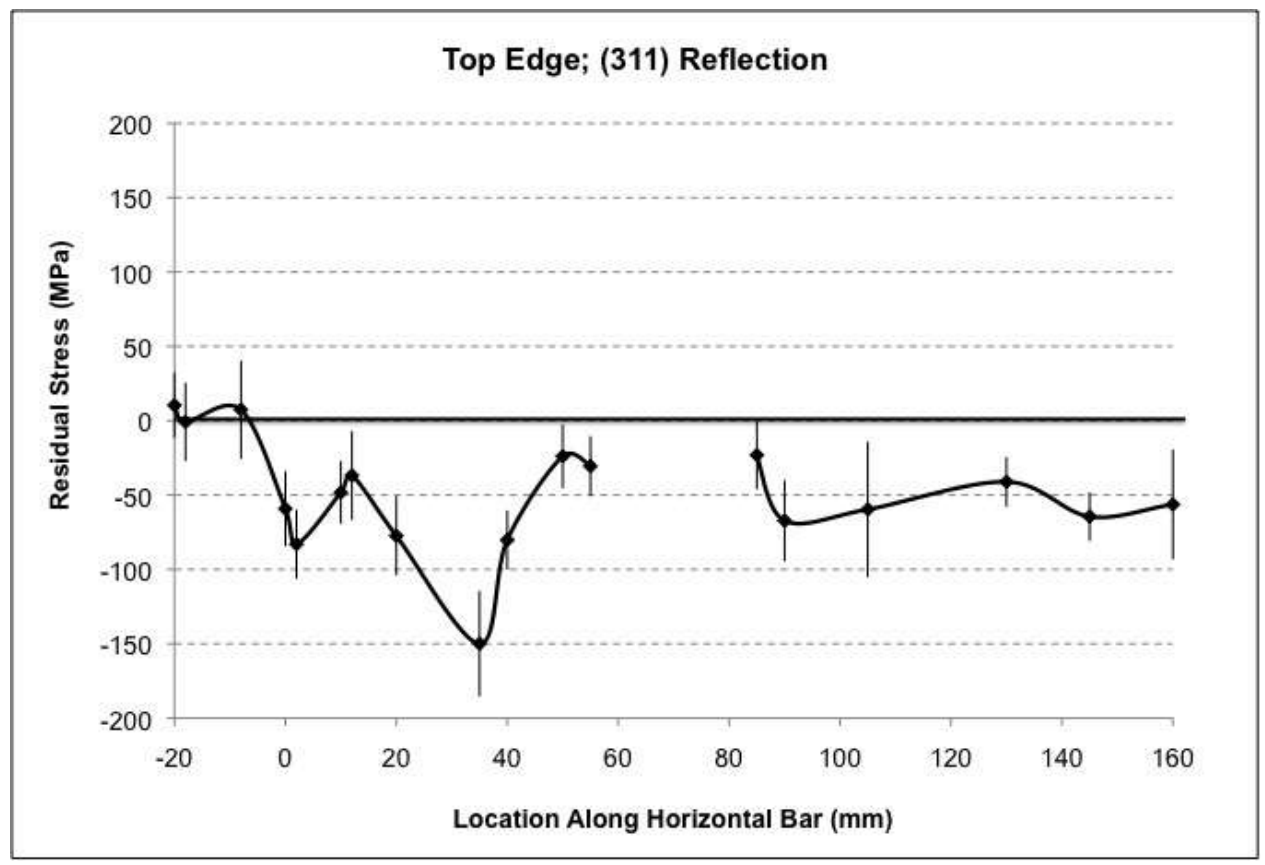

a)

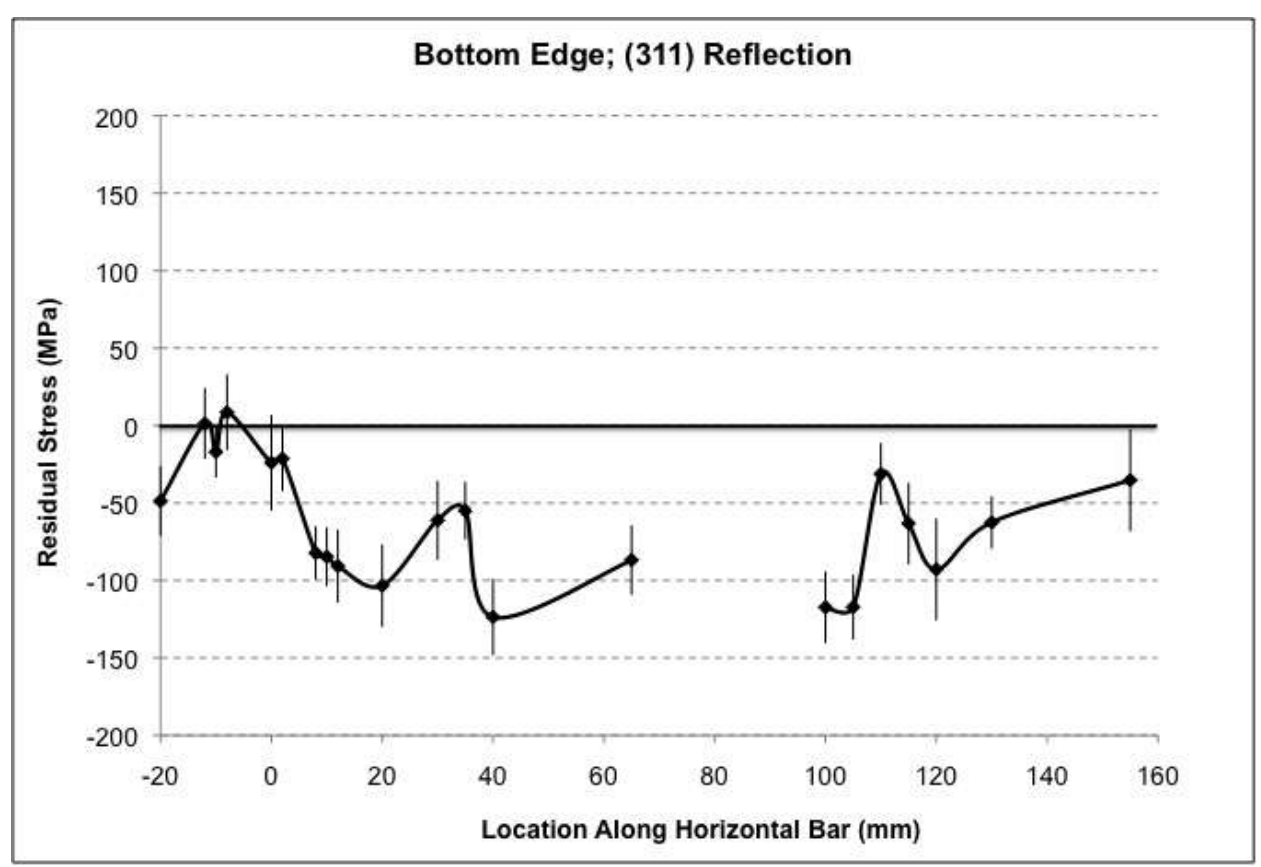

b)

Figure 4-52. Profiles of $\sigma_{x}$ along the (a) top edge and (b) bottom edge of the horizontal bar for the (311) reflection of the unrefined $\mathrm{B206}$ alloy. 


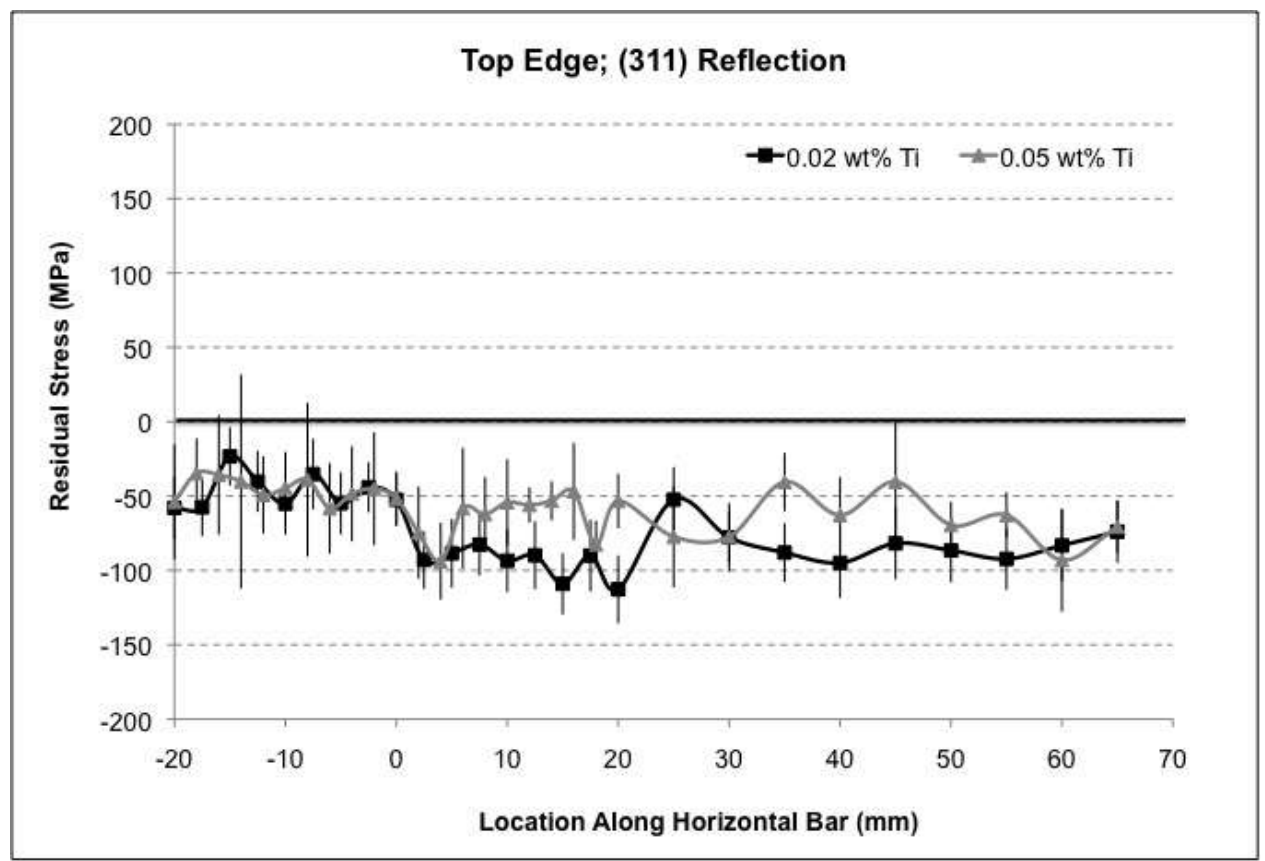

a)

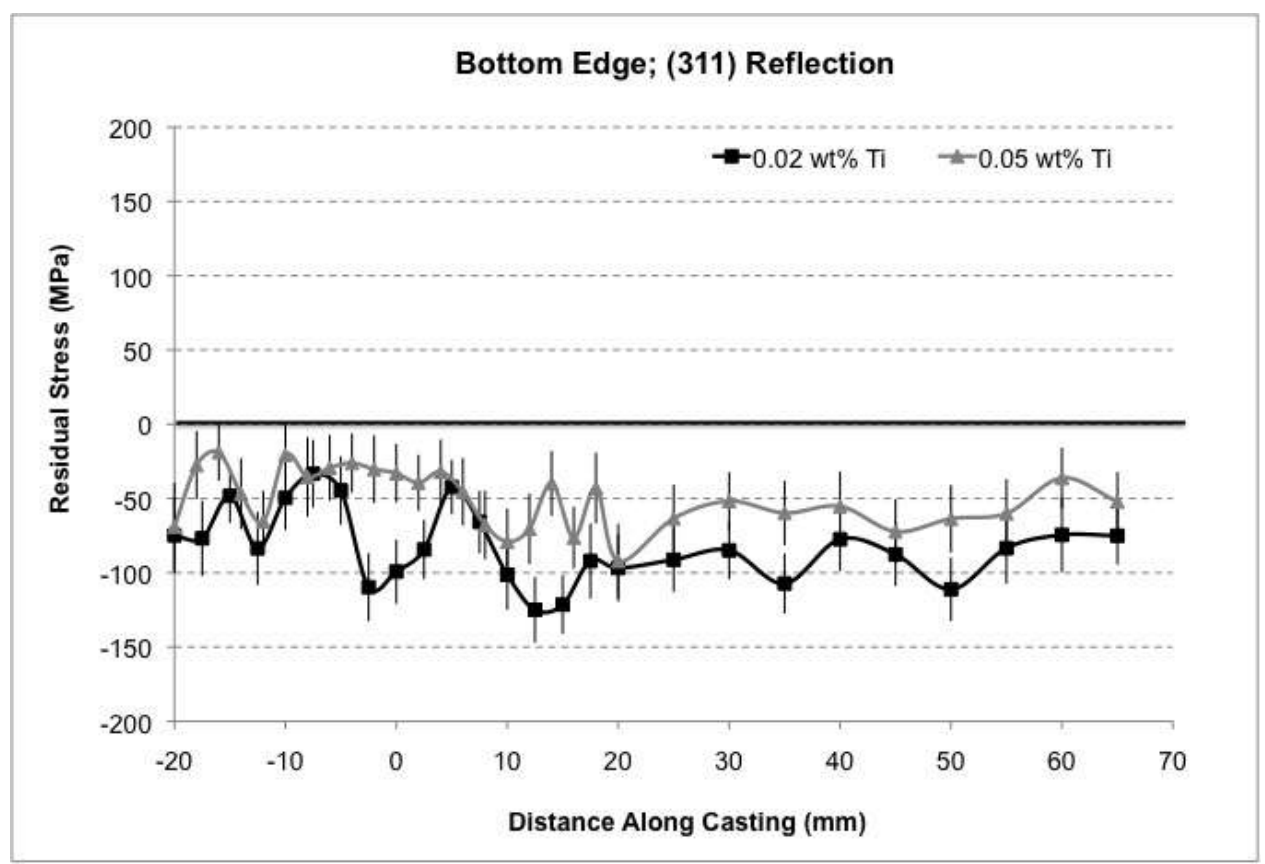

b)

Figure 4-53. Profiles of $\sigma_{x}$ along the a) top edge and b) bottom edge of the horizontal bar for the (311) reflection of the $0.02 \mathrm{wt} \% \mathrm{Ti}$ and $0.05 \mathrm{wt} \% \mathrm{Ti}$ alloys. 


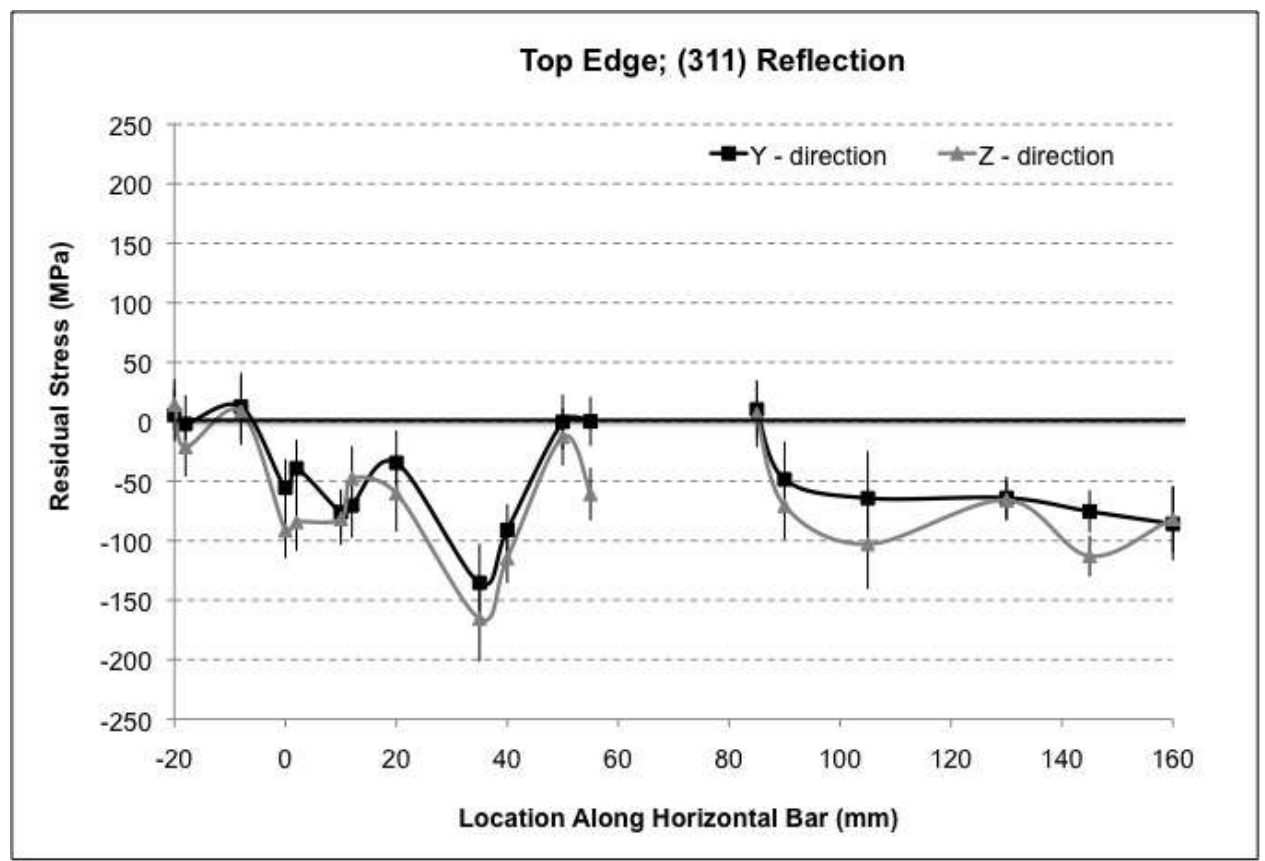

a)

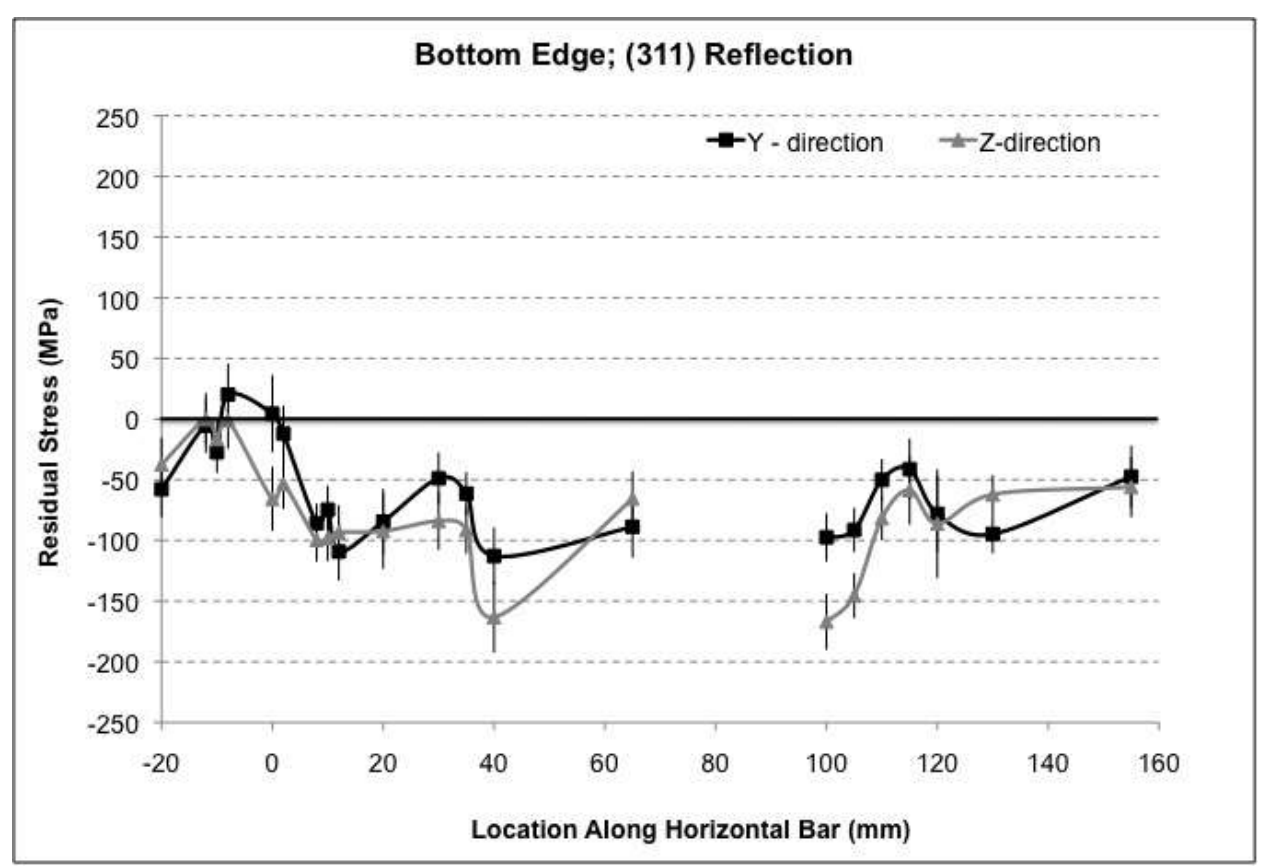

b)

Figure 4-54. Profiles of $\sigma_{y}$ and $\sigma_{z}$ along the a) top edge and b) bottom edge of the horizontal bar for the (311) reflection of the unrefined B206 alloy. 


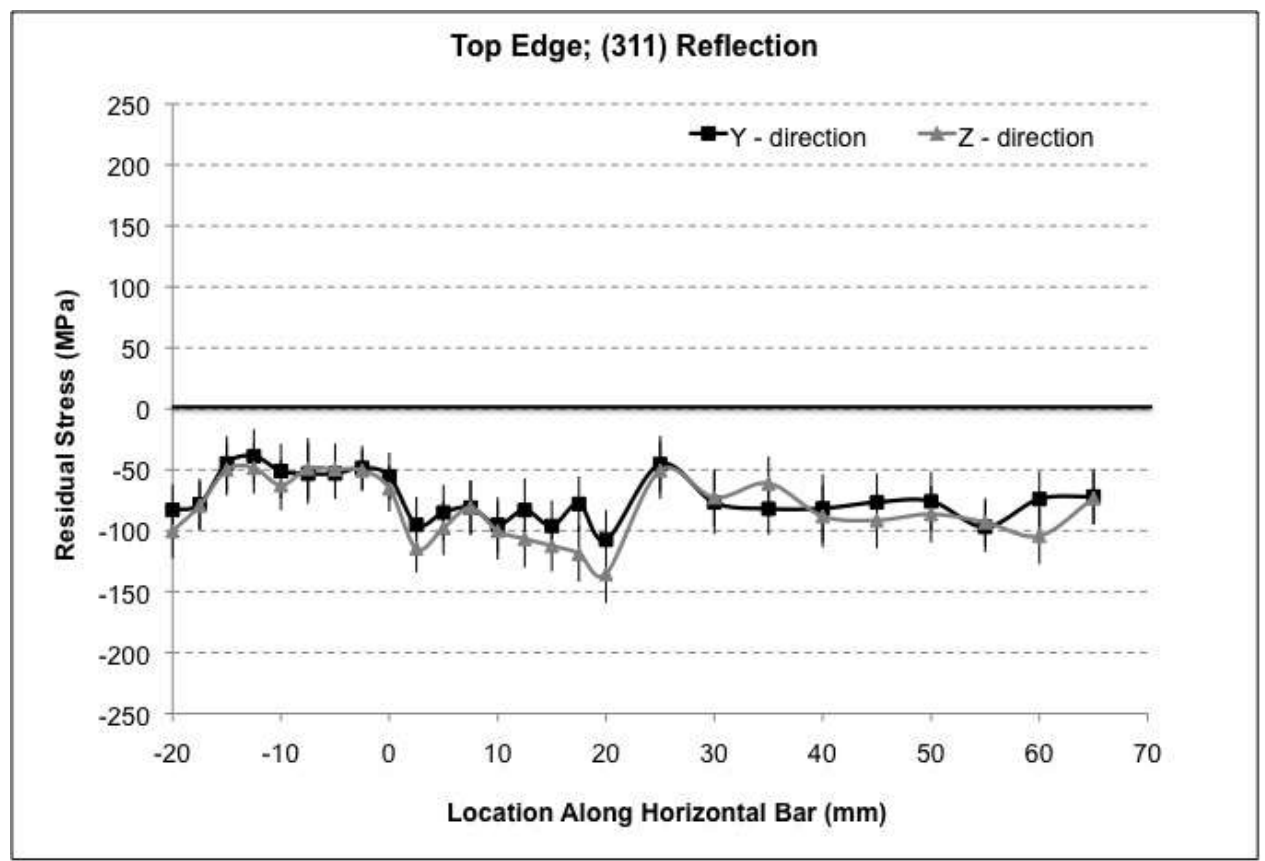

a)

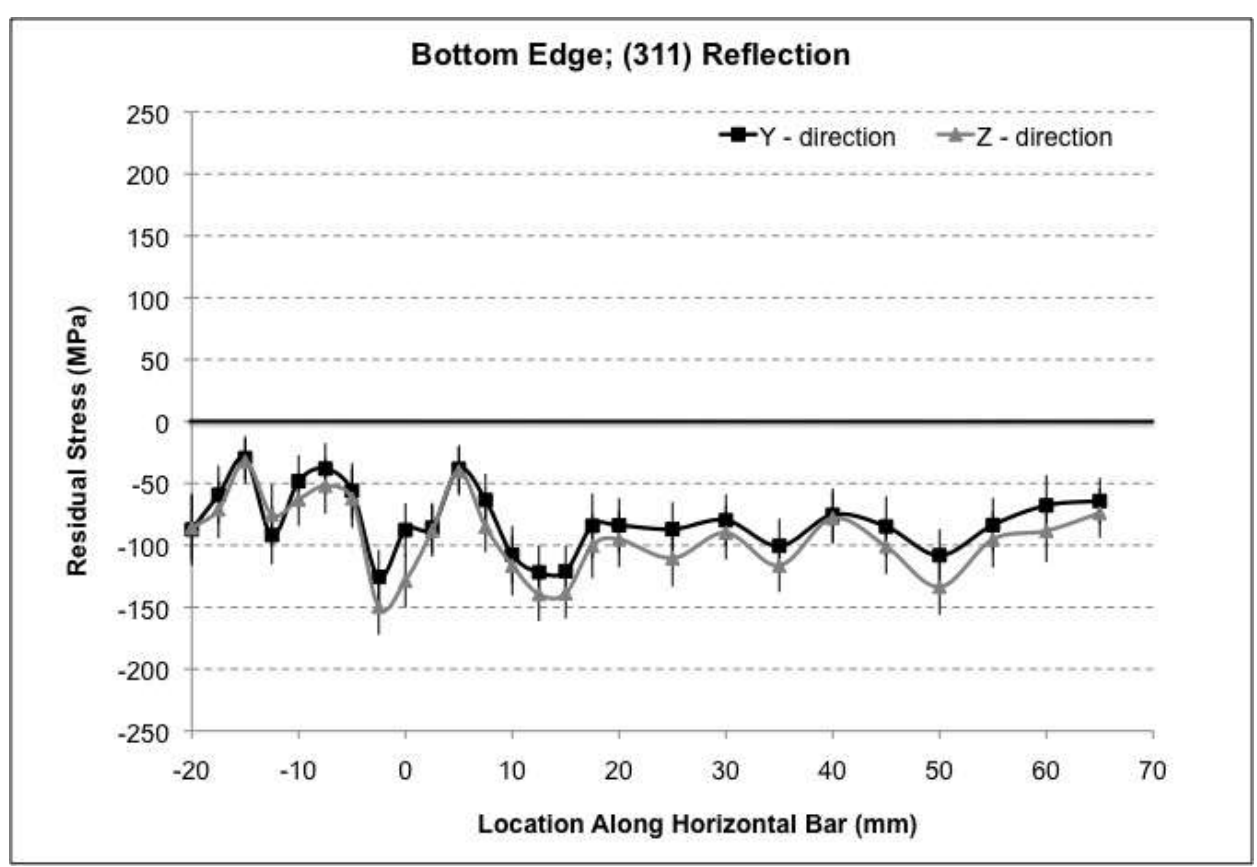

b)

Figure 4-55. Profiles of $\sigma_{y}$ and $\sigma_{z}$ along the a) top edge and b) bottom edge of the horizontal bar for the (311) reflection of the $0.02 \mathrm{wt} \% \mathrm{Ti}$ alloy. 


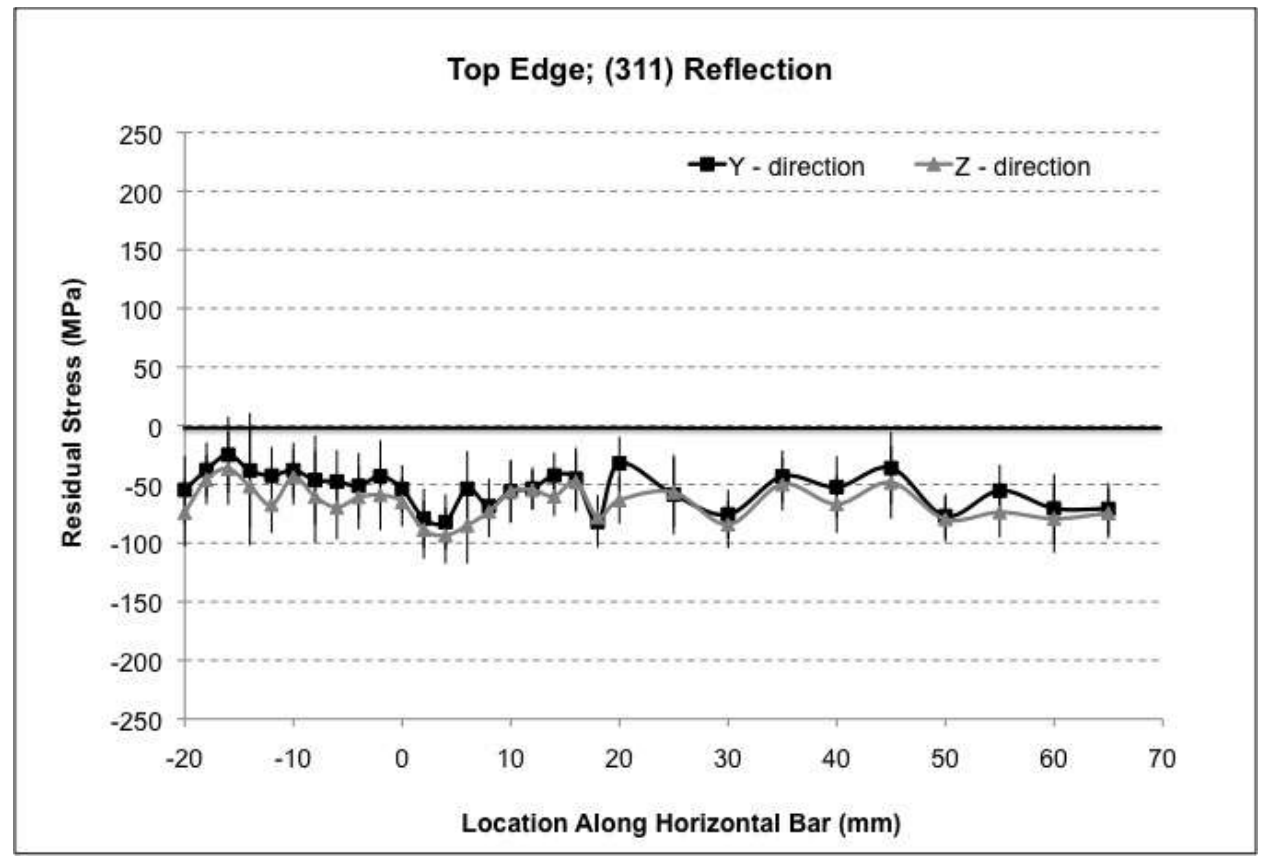

a)

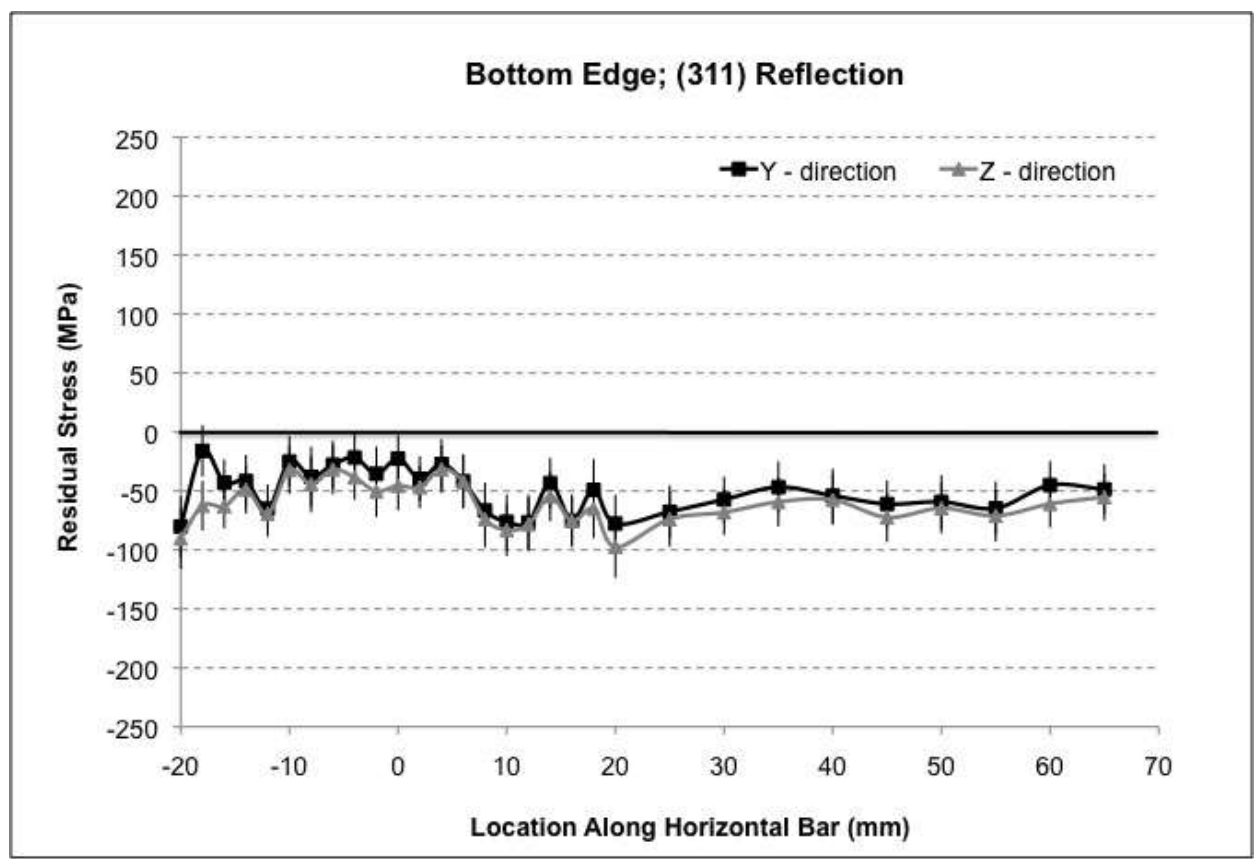

b)

Figure 4-56. Profiles of $\sigma_{y}$ and $\sigma_{z}$ along the a) top edge and b) bottom edge of the horizontal bar for the (311) reflection of the $0.05 \mathrm{wt} \% \mathrm{Ti}$ alloy. 


\section{Chapter 5 - In Situ Analysis of Hot Tearing in B206}

The results of the in situ casting experiments carried out in this research are presented in this chapter. The severity of hot tearing present on the castings is illustrated in Section 5.1. The results of thermal analysis and the onset temperatures of hot tearing of each B206 casting condition are presented in Section 5.2. Finally, casting microstructure analysis and the results of in situ neutron diffraction solidification analysis are presented in the following two sections, respectively.

\subsection{Observation of Hot Tears on Casting Surfaces}

The casting surfaces of the B206 alloy are shown in Figure 5-1 for each casting condition. Figures 5-1a-c illustrate the effect of mold temperature on hot tearing in the unrefined B206. A hot tear was present at the sprue-rod junction for all mold temperatures (i.e. $250{ }^{\circ} \mathrm{C}, 325{ }^{\circ} \mathrm{C}$ and $400{ }^{\circ} \mathrm{C}$ ). As the mold temperature increased to $400{ }^{\circ} \mathrm{C}$, however, the severity of hot tearing was significantly reduced, as shown in Figure 5-1c. Additions of $\mathrm{Ti}$ at this mold temperature effectively eliminated surface hot tears in B206, as no tears were present on the casting surface of the $0.02 \mathrm{wt} \% \mathrm{Ti}$ (Figure 5-1d) and $0.05 \mathrm{wt} \%$ Ti alloys (Figure 5-1e).

\subsection{Force-Temperature-Time Curves}

The force-time and temperature-time curves for each casting condition are presented in Figures 5-2 and 5-3 for the unrefined and refined alloys, respectively. The temperature-time curves were used to calculate the cooling rate and solidification time for each condition. The $250{ }^{\circ} \mathrm{C}, 325^{\circ} \mathrm{C}$ and $400{ }^{\circ} \mathrm{C}$ mold temperatures corresponded to cooling rates of $9.0{ }^{\circ} \mathrm{C} / \mathrm{s}, 7.8^{\circ} \mathrm{C} / \mathrm{s}$ and $5.7{ }^{\circ} \mathrm{C} / \mathrm{s}$, respectively for the unrefined B206 alloy. In the case of the refined alloys, the addition of Ti did not significantly impact the cooling rate (at $400{ }^{\circ} \mathrm{C}$ mold temperature) as they were measured as $5.9^{\circ} \mathrm{C} / \mathrm{s}$ and $6.2^{\circ} \mathrm{C} / \mathrm{s}$ for the $0.02 \mathrm{wt} \% \mathrm{Ti}$ and $0.05 \mathrm{wt} \%$ Ti alloys, respectively.

\footnotetext{
$\$$ Much of the data presented in this chapter has been published in: D’Elia, F., Ravindran, C., Sediako, D., Kainer, K.U. and Hort, N., "Hot Tearing Mechanisms of B206 Aluminum-Copper Alloy." Materials and Design. Vol. 64 (2014): 44-55; D'Elia, F., Ravindran, C., Sediako, D. and Donaberger, R., "Solidification Analysis of an Al-5 wt.-\% Cu Alloy Using In Situ Neutron Diffraction.” Canadian Metallurgical Quarterly. (accepted: July 2014).
} 


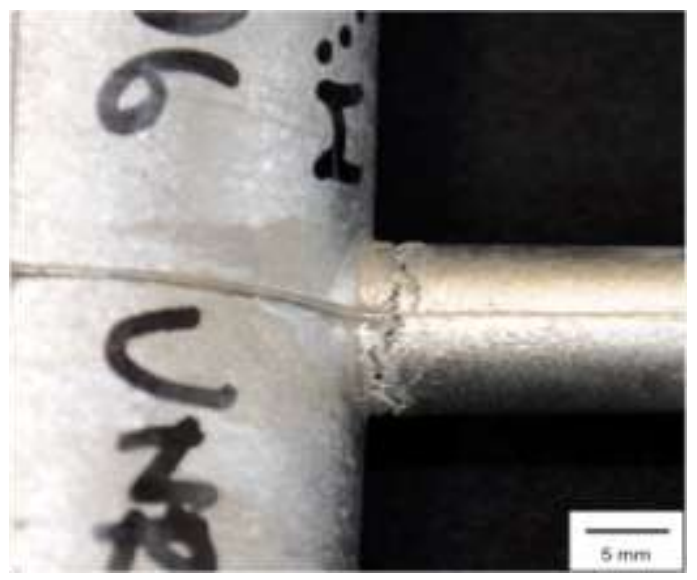

a)

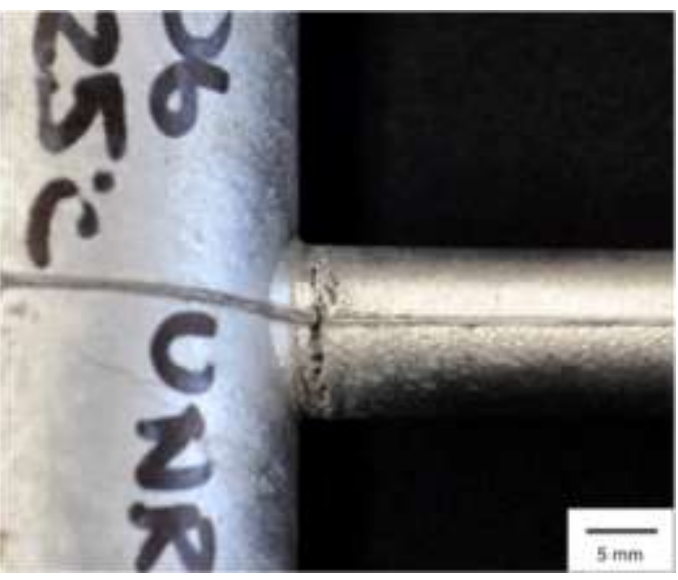

b)

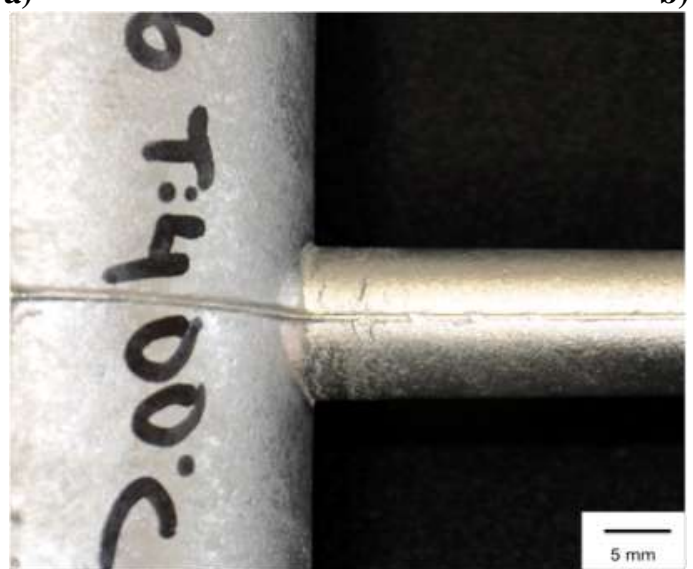

c)

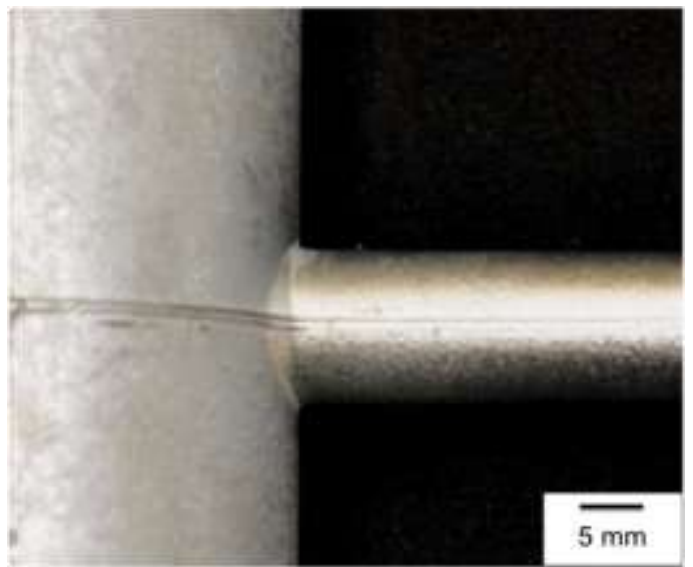

d)

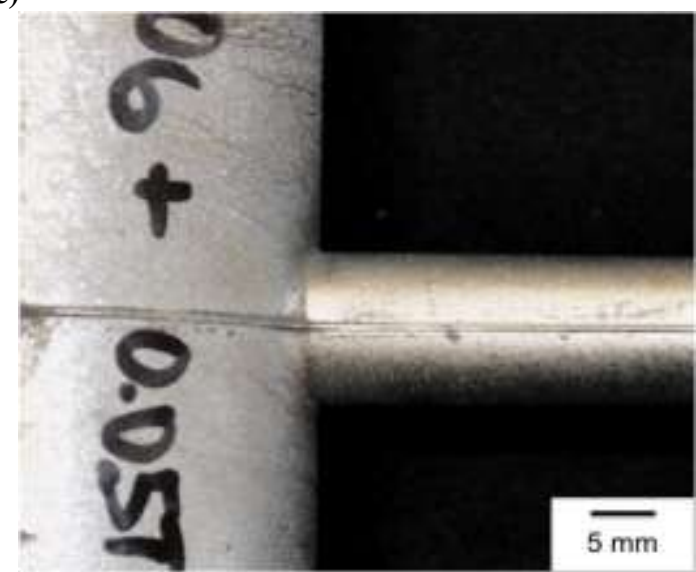

e)

Figure 5-1. The casting surfaces of a) unrefined $\mathrm{B} 206$ at $250{ }^{\circ} \mathrm{C}$ mold temperature; b) unrefined $\mathrm{B206}$ at $325{ }^{\circ} \mathrm{C}$ mold temperature; c) unrefined $\mathrm{B} 206$ at $400{ }^{\circ} \mathrm{C}$ mold temperature; d) $0.02 \mathrm{wt} \%$ Ti alloy at $400{ }^{\circ} \mathrm{C}$ mold temperature and e) $0.05 \mathrm{wt} \% \mathrm{Ti}$ alloy at $400{ }^{\circ} \mathrm{C}$ mold temperature.

The solidification time significantly increased in the unrefined alloy with higher mold temperatures, as expected. The solidification time was measured at $103 \mathrm{~s}, 199 \mathrm{~s}$ and $398 \mathrm{~s}$ for the 
three mold temperatures, respectively. On the other hand, the addition of $\mathrm{Ti}$ was not seen to significantly influence the solidification time in B206.

The hot tears present in the unrefined B206 alloys were confirmed by the dip in the force curves, as shown in Figures 5-2. Section 3.3.3 described the methodology for which the onset temperature of hot tearing was determined. The corresponding hot tear initiation temperatures for the $250{ }^{\circ} \mathrm{C}, 325{ }^{\circ} \mathrm{C}$ and $400{ }^{\circ} \mathrm{C}$ mold temperatures were measured at $619{ }^{\circ} \mathrm{C}, 602{ }^{\circ} \mathrm{C}$ and $637^{\circ} \mathrm{C}$, respectively. The associated fraction solid was determined by neutron diffraction and is presented later (Section 5.4.4). Further inspection of the force curve in Figure 5-2c for the $400{ }^{\circ} \mathrm{C}$ mold temperature, suggests that a secondary crack may have occurred in the alloy, as a second relief in the force curve is observed. The temperature corresponding to this secondary crack was $610^{\circ} \mathrm{C}$. Inspection of the casting surface in Figure 5-1c confirms the presence of two separate fine tears. The recorded force curves also reflect the hot tear propagation. Since crack propagation is a continuous stress releasing process, the faster the crack propagates the sharper the force drops [32]. This was the case for Figure 5-2a and b with mold temperatures of $250{ }^{\circ} \mathrm{C}$ and $325^{\circ} \mathrm{C}$, respectively. However, for Figure 5-2c the force drop was significantly shorter. Such observations on the force-time curves were in agreement to the hot tearing severity of the alloys observed in Figure 5-1.

The force-temperature-time curves for the $0.02 \mathrm{wt} \% \mathrm{Ti}$ and $0.05 \mathrm{wt} \% \mathrm{Ti}$ alloys are shown in Figure 5-3a and b, respectively. A hot tear was not present on the casting surface of either alloy (Figure 5-1). As a result, no relief in contraction force was visible on the force-time curve for either alloy. Instead, the curves illustrate a continuous increase in relative tension as the casting solidified. Thus, the force-time curves showed good correlation to the hot tearing susceptibility of the refined alloys as well. 


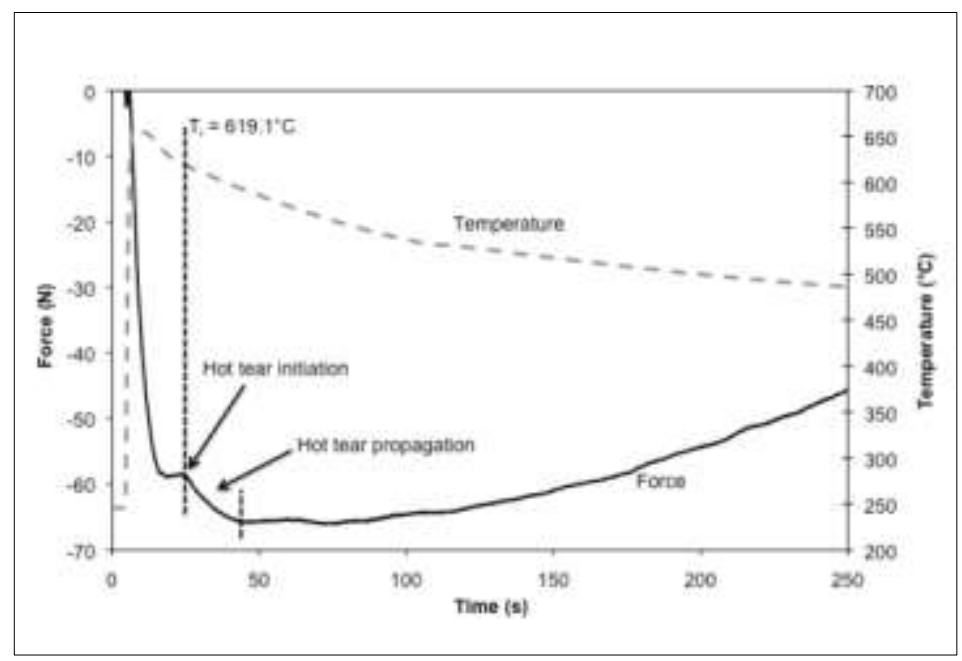

a)

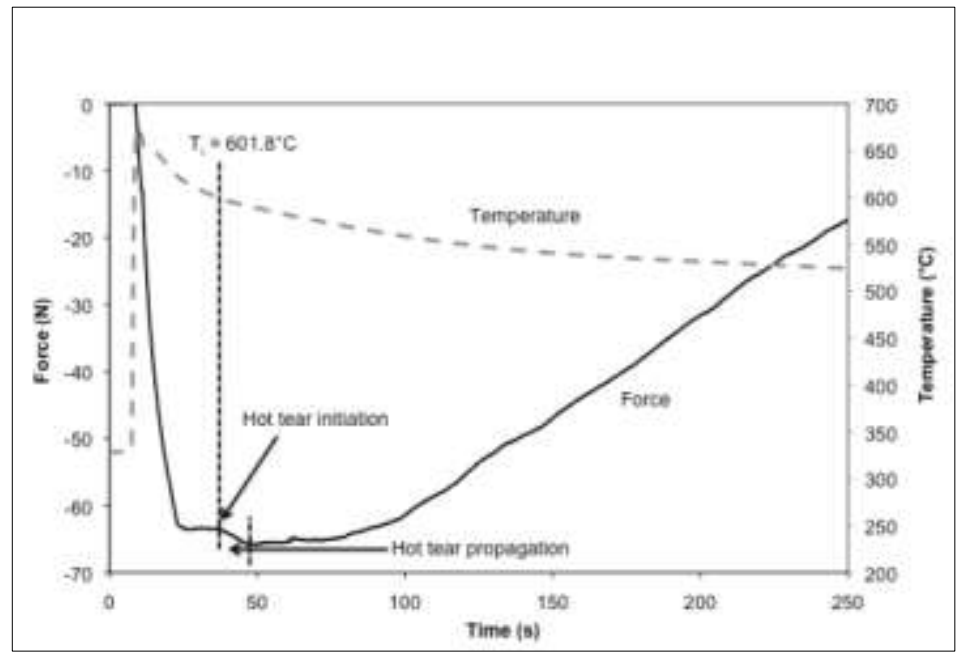

b)

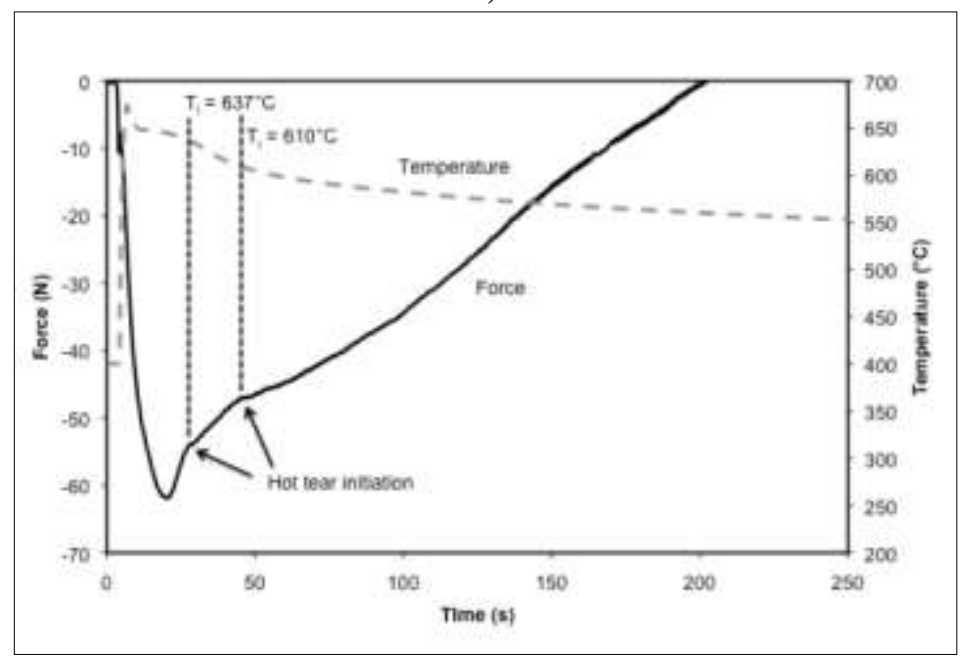

c)

Figure 5-2. Force-temperature-time curves for unrefined $\mathrm{B206}$ at a) $250{ }^{\circ} \mathrm{C}$; b) $325{ }^{\circ} \mathrm{C}$ and c) $400{ }^{\circ} \mathrm{C}$ mold temperatures. 


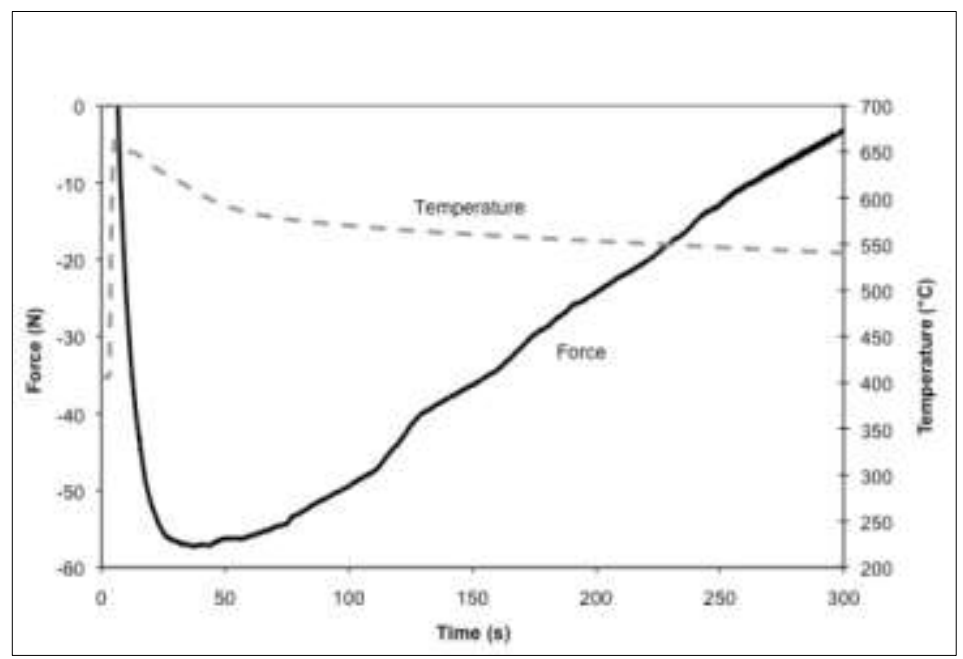

a)

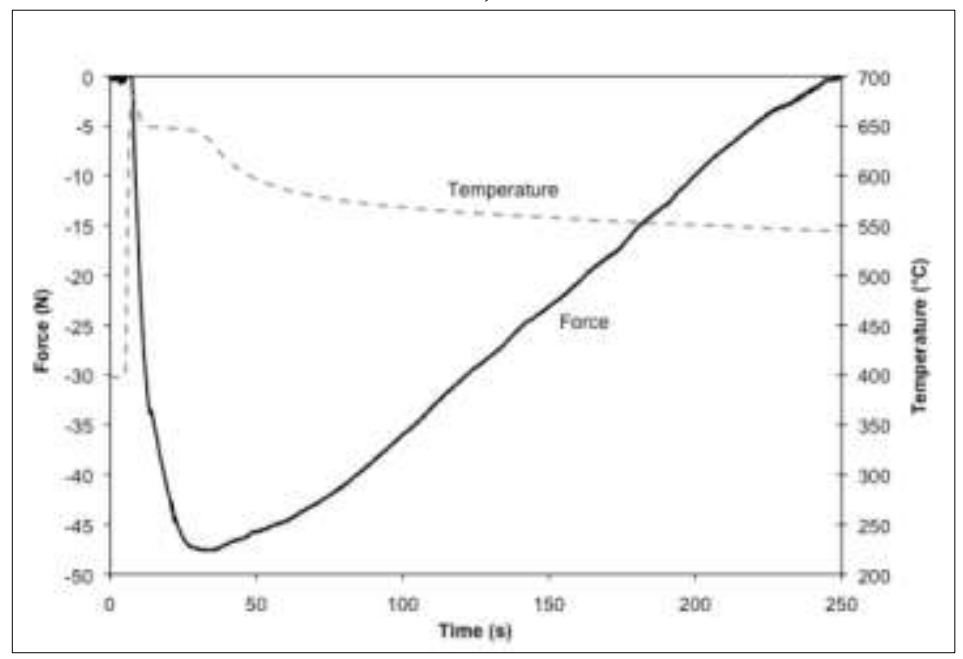

b)

Figure 5-3. Force-temperature-time curves for a) 0.02 wt $\%$ Ti alloy and b) 0.05 wt $\%$ Ti alloy.

Repeat trials were carried out and are presented in Appendix 5 (Figures A.5-1 to A.5-5). The trends of the force curves were similar to those presented in this section for the respective conditions. However, minor variations in onset temperature of hot tearing were recorded. For instance, in the unrefined alloy, the hot tears were found to initiate at $638{ }^{\circ} \mathrm{C}, 611^{\circ} \mathrm{C}$ and $637{ }^{\circ} \mathrm{C}$ for the $250{ }^{\circ} \mathrm{C}, 325{ }^{\circ} \mathrm{C}$ and $400{ }^{\circ} \mathrm{C}$ mold temperatures, respectively. Such differences in temperature were expected due to the unpredictable nature of hot tearing. In the case of the refined alloys, again, no hot tears were present and thus, no relief in contraction force was seen on the force curve for either alloy. 


\subsection{Casting Microstructure Analysis}

Optical and scanning electron microscopy were carried out for the in situ B206 castings in order to confirm the effect of Ti additions on grain size and microstructure observed for the ex situ castings. The grain size measurements and resulting grain morphology of the five conditions (presented in Section 5.1) are presented in Section 5.3.1, while the distribution and morphology of secondary phases are presented in the following section.

\subsubsection{Grain Size and Morphology}

The grain size measurements for the B206 alloy are presented in Figure 5-4 for each casting condition. An increase in average grain size was evident for higher mold temperatures. The average grain size was seen to steadily increase from $716 \pm 117 \mu \mathrm{m}$ to $935 \pm 159 \mu \mathrm{m}$ and finally to $1167 \pm 245 \mu \mathrm{m}$ for the three $\left(250^{\circ} \mathrm{C}, 325^{\circ} \mathrm{C}\right.$ and $\left.400{ }^{\circ} \mathrm{C}\right)$ mold temperatures, respectively. This increase in grain size was likely attributed to the lower cooling rates associated with higher mold temperatures. In contrast, addition of Ti resulted in a significant reduction in average grain size, as shown in Figure 5-4. With the addition of $0.02 \mathrm{wt} \% \mathrm{Ti}$, the average alloy grain size reduced from $1167 \pm 245 \mu \mathrm{m}$ to $97 \pm 19 \mu \mathrm{m}$. Further additions of Ti to $0.05 \mathrm{wt} \%$ decreased average alloy grain size to $72 \pm 7 \mu \mathrm{m}$. Hence, the results of grain size measurements for the in situ castings showed good agreement to those of the ex situ castings.

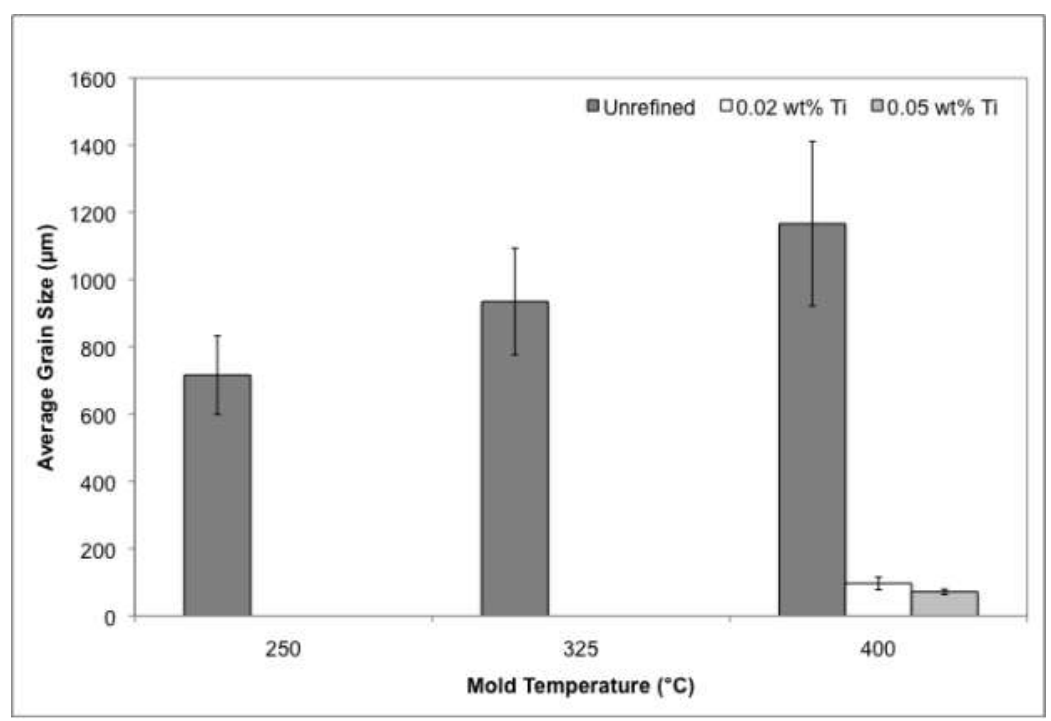

Figure 5-4. Effect of mold temperature and Ti level on grain size in B206. 
Opposite trends were observed in the hot tearing susceptibility of B206 with respect to the effect of mold temperature and Ti addition levels. Higher mold temperatures resulted in reduced hot tearing severity but larger grain sizes. In contrast, the addition of Ti resulted in both a significant reduction in grain size and hot tearing severity. The grain morphology of each alloy was examined to help develop reasoning for this conflicting outcome.

The effect of mold temperature on the grain morphology of B206 is shown in Figure 5-5. A finer grain size is evident with lower mold temperatures. However, despite the decrease in grain size, the grain morphology for all three conditions remained dendritic. The micrographs also illustrate the presence of columnar dendrites, which propagated from the mold wall towards the centre of the casting. Columnar grain growth was more pronounced at higher mold temperatures, as slower cooling and enhanced solidification time enabled more time for grain growth.

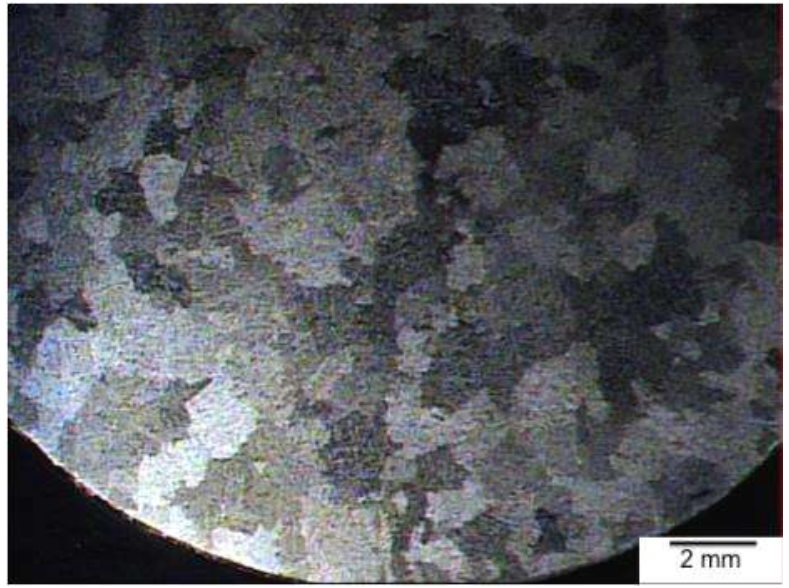

a)

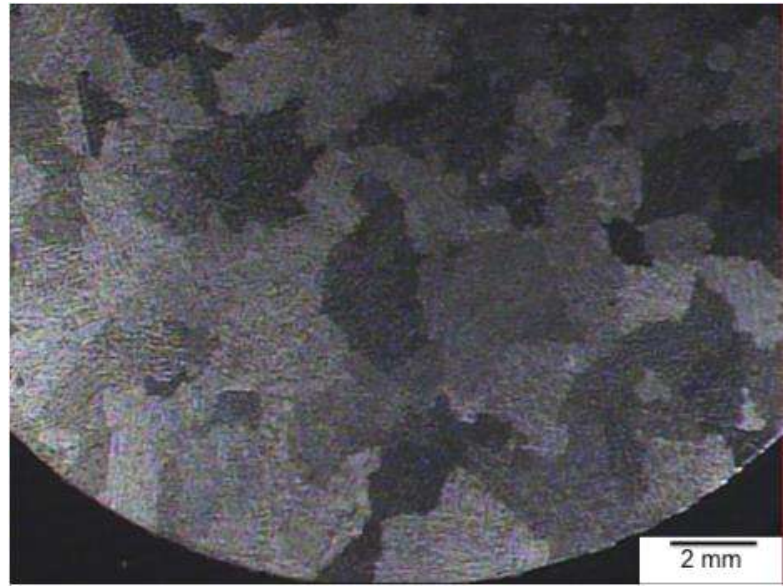

b)

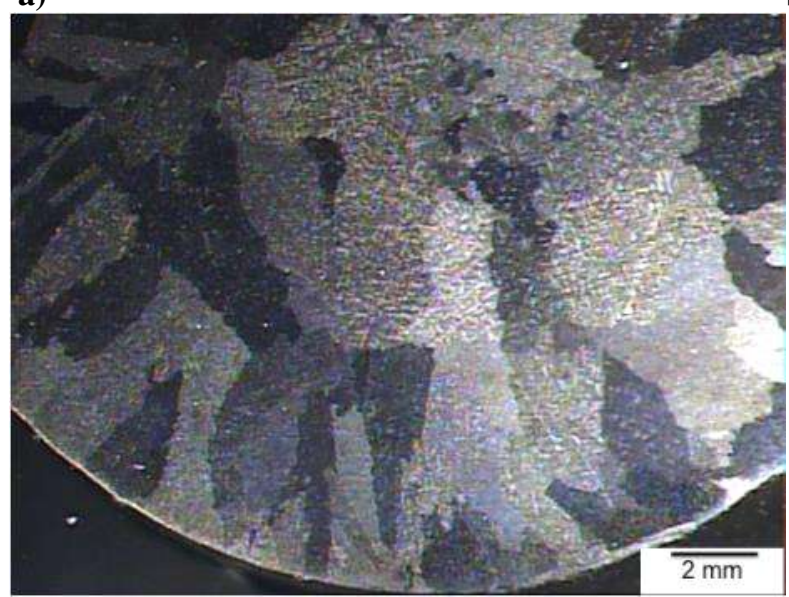

c)

Figure 5-5. Grain morphology of unrefined B206 at mold temperatures of a) $250{ }^{\circ} \mathrm{C}$; b) $325{ }^{\circ} \mathrm{C}$ and c) $400{ }^{\circ} \mathrm{C}$. 
The effect of Ti on grain structure is illustrated in Figure 5-6. Titanium additions were not only effective at reducing alloy grain size but also at transforming the alloy grain morphology. The coarse dendritic structure of the unrefined B206 alloy is shown in Figure 5-6a. Addition of $0.02 \mathrm{wt} \% \mathrm{Ti}$ resulted in a significant transformation to a finer and more equiaxed structure, as shown in Figure 5-6b. However, the grain structure was not completely globular for the $0.02 \mathrm{wt} \% \mathrm{Ti}$ alloy, as there was evidence of small dendrite arms. With addition of $0.05 \mathrm{wt} \% \mathrm{Ti}$, the grain morphology transformed to a more globular structure (Figure 5-6c), as significantly less branching between grains was observed on the micrograph. Thus, the grain morphologies of the in situ castings also showed good agreement to those of the ex situ casting experiments.

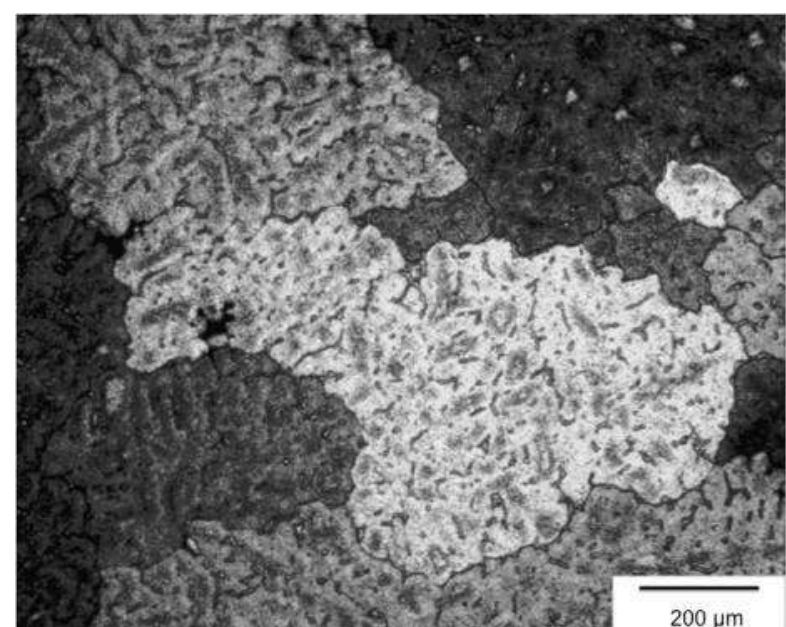

a)
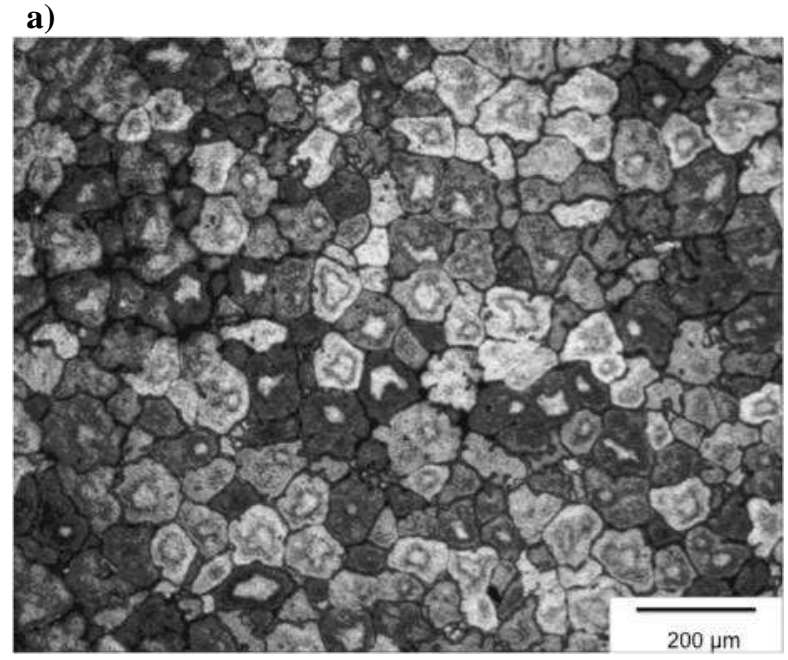

c)

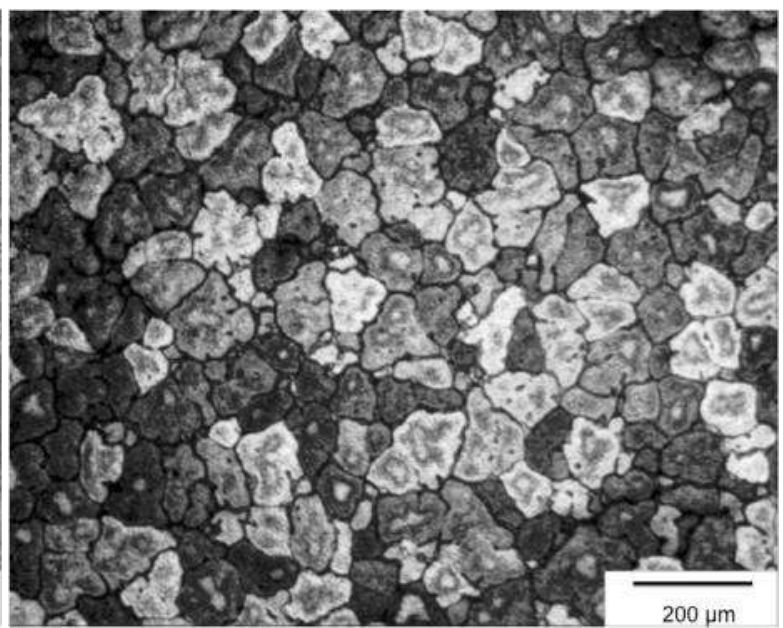

b)

Figure 5-6. Grain morphology of the a) unrefined B206 alloy; b) $0.02 \mathrm{wt} \% \mathrm{Ti}$ alloy and c) $0.05 \mathrm{wt} \% \mathrm{Ti}$ alloy. 


\subsubsection{General Alloy Microstructure}

Scanning electron microscopy was carried out to examine the effect of mold temperature on the distribution and morphology of the $\mathrm{Al}_{2} \mathrm{Cu}$ intermetallic. Figure 5-7 illustrates the microstructure for the three mold temperatures investigated. EDX analysis in Figure 5-8 confirms the presence of $\mathrm{Al}_{2} \mathrm{Cu}$ intermetallic. Inspection of the micrographs suggests that higher mold temperatures did not have a significant effect on the distribution and morphology of $\mathrm{Al}_{2} \mathrm{Cu}$. Results of image analysis, shown in Figure 5-9, also confirm that the average amount of intermetallic did not significantly change for different mold temperatures. Therefore, the reduction of hot tearing severity with higher mold temperatures in the unrefined B206 alloy was likely attributed solely to the enhanced time available for liquid feeding, as a result of the increase in solidification time.

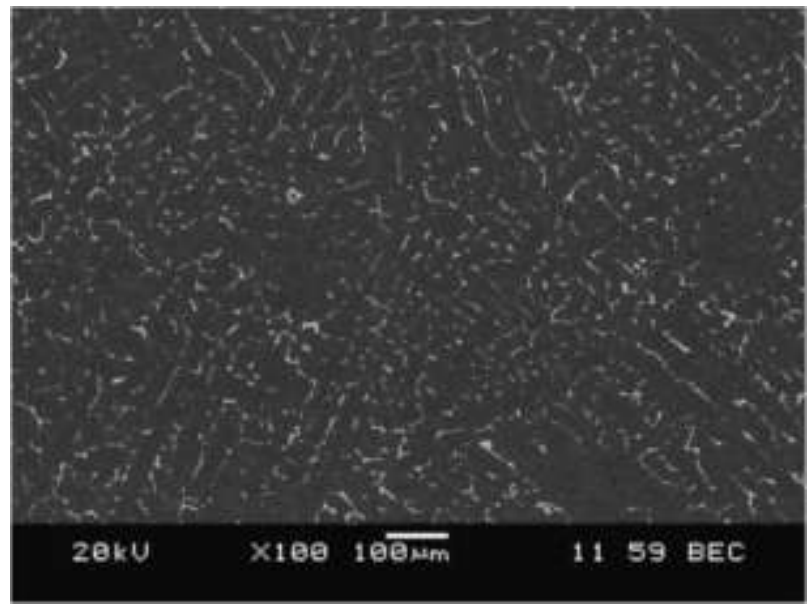

a)

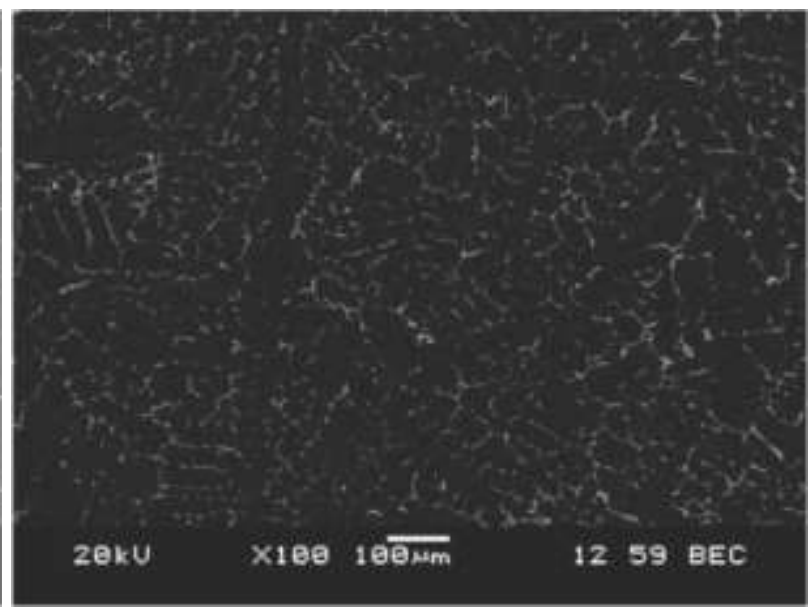

b)

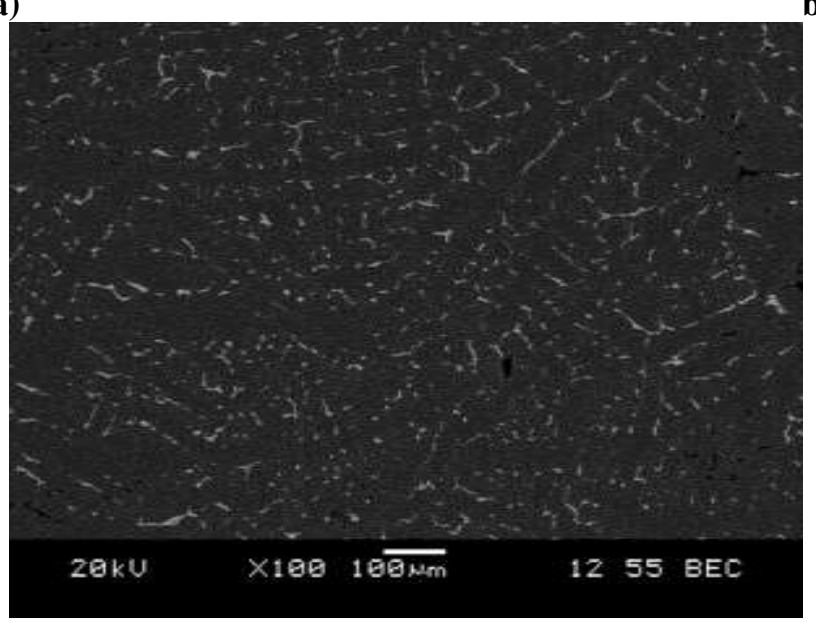

c)

Figure 5-7. Effect of a) $250{ }^{\circ} \mathrm{C}$; b) $325{ }^{\circ} \mathrm{C}$ and c) $400{ }^{\circ} \mathrm{C}$ mold temperature on $\mathrm{Al}_{2} \mathrm{Cu}$ in unrefined $\mathrm{B206}$. 


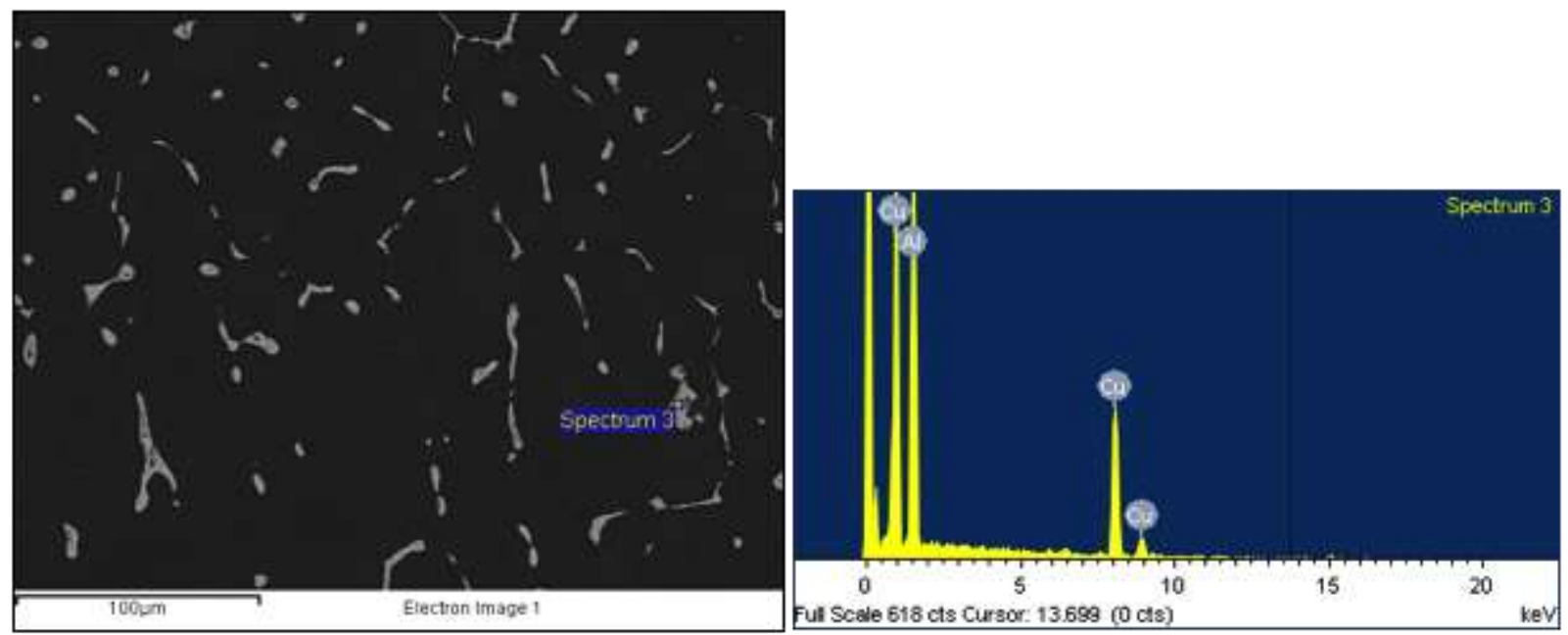

Figure 5-8. EDX analysis confirming the $\mathrm{Al}_{2} \mathrm{Cu}$ intermetallic.

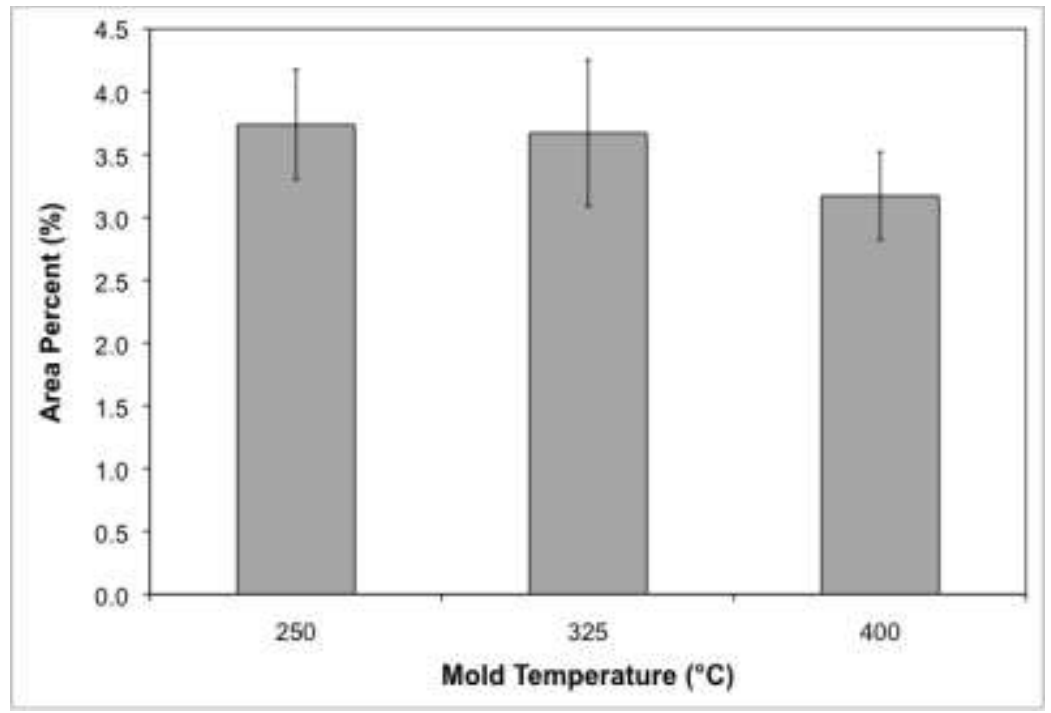

Figure 5-9. Effect of mold temperature on percent area of $\mathrm{Al}_{2} \mathrm{Cu}$.

The effect of $\mathrm{Ti}$ additions on the distribution of $\mathrm{Al}_{2} \mathrm{Cu}$ is illustrated in Figure 5-10. With additions of $\mathrm{Ti}$, the subsequent decrease in grain size resulted in increased grain boundary area for the refined alloys. This enabled a more uniform distribution of $\mathrm{Al}_{2} \mathrm{Cu}$ intermetallic along the grain boundary regions, which is depicted in Figure 5-10a and b for the $0.02 \mathrm{wt} \% \mathrm{Ti}$ and $0.05 \mathrm{wt} \% \mathrm{Ti}$ alloys, respectively. In contrast, the coarse columnar grains observed in the unrefined alloy, resulted in a less homogeneous distribution, as shown in Figure 5-7. Image analysis confirmed that the amount of intermetallic was virtually unaffected by additions of Ti. A large disparity in the size of the regions containing intermetallic were seen in the unrefined alloy, as large pockets of intermetallic were visible between coarse primary dendrites arms, while 
smaller pockets of intermetallic were found between finer secondary arms. Such variance in $\mathrm{Al}_{2} \mathrm{Cu}$ distribution between the unrefined and refined alloys suggests that late stage feeding of liquid varied between the alloys as well. In turn, shrinkage was likely more accommodated by uniform liquid feeding in the refined grain structures making them less prone to hot tear formation.

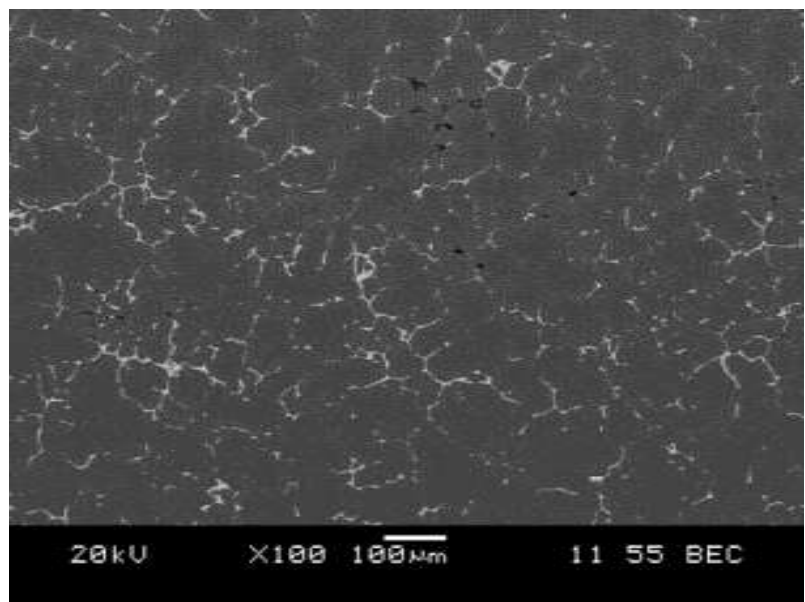

a)

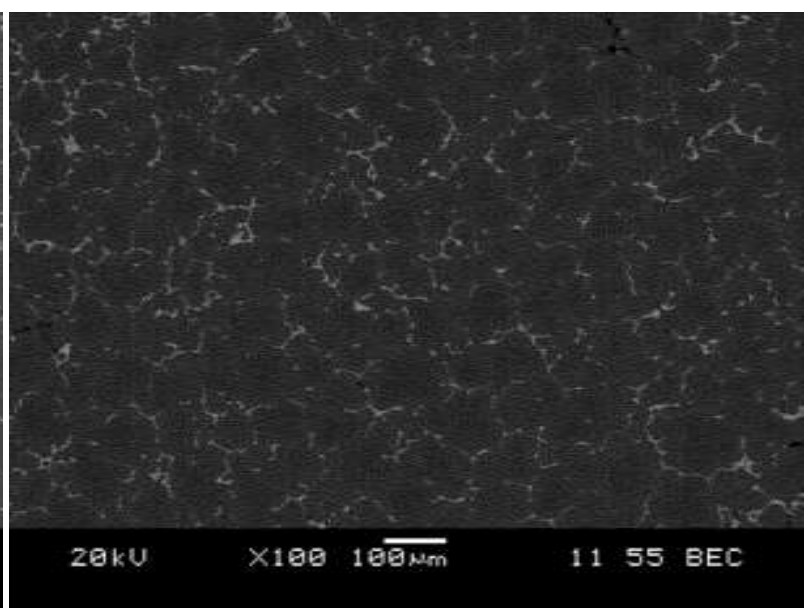

b)

Figure 5-10. Effect of $\mathrm{Ti}$ on $\mathrm{Al}_{2} \mathrm{Cu}$ intermetallic in a) $0.02 \mathrm{wt} \% \mathrm{Ti}$ alloy and b) $0.05 \mathrm{wt} \% \mathrm{Ti}$ alloy.

Image analysis was also carried out to determine the effect of grain refinement on the amount of $\mathrm{Al}_{2} \mathrm{Cu}$ intermetallic. The results in Figure 5-11 confirm that the area percent of $\mathrm{Al}_{2} \mathrm{Cu}$ was virtually unaffected by additions of Ti.

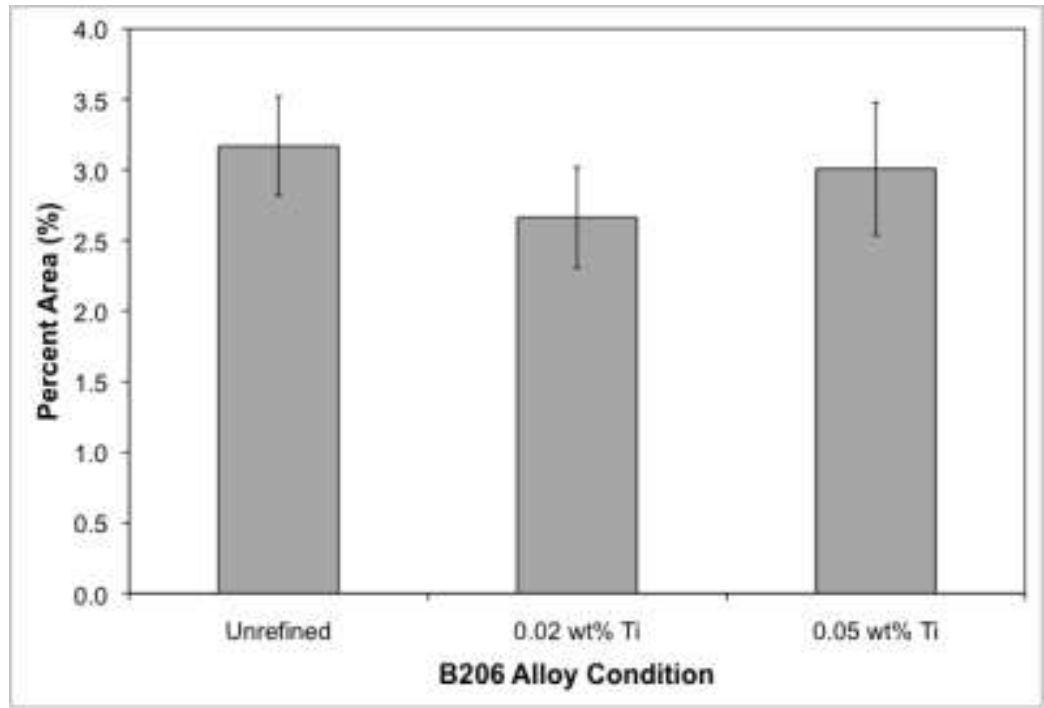

Figure 5-11. Effect of Ti on percent area of $\mathrm{Al}_{2} \mathrm{Cu}$. 


\subsection{In Situ Neutron Diffraction Solidification Analysis}

In situ neutron diffraction solidification analysis was carried out to gain an understanding on the solidification kinetics and the development of microstructure in B206. The in situ neutron diffraction experiments began with a scan of an empty (i.e. without sample material) graphite crucible. The crucible was attached to the steel holder that was a part of the solidification cell, as shown in Figure 3-18 (Section 3.4.3). The control K-type thermocouple cladded in stainless steel sheath was placed inside the protective graphite sleeve and inserted into the crucible as per usual experiment practice. This no-sample scan enabled clear delineation of the 'background' that contributed to the diffraction pattern by the graphite and steel. The 'no-sample background' was then subtracted from the diffraction pattern generated in the actual sample scan. Thus, clear 'sample-only' diffraction patterns were used for the peaks' intensity analysis [81].

In this section, the FactSage simulations are first presented in Section 5.4.1, followed by the results of in situ neutron diffraction experiments in Section 5.4.2 and 5.4.3. Finally, the section concludes with a discussion on the role of microstructure on hot tear formation in B206.

\subsubsection{FactSage Simulation}

FactSage simulation enabled calculation of the cooling path for the binary Al-5 wt $\% \mathrm{Cu}$ alloy. The thermodynamic calculations are presented in Figure 5-12 for equilibrium solidification mode. The results are presented as a plot of composition, in wt $\%$, vs. temperature, in ${ }^{\circ} \mathrm{C}$. The calculation was carried out between the temperature interval of $700-420{ }^{\circ} \mathrm{C}$. Initially, in the range of $700-650{ }^{\circ} \mathrm{C}$ the liquid is a mixture of $5 \mathrm{wt} \% \mathrm{Cu}$ and $95 \mathrm{wt} \%$ Al. At $650{ }^{\circ} \mathrm{C}$ (i.e. the liquidus temperature) the $\alpha$-Al solid solution begins to precipitate. The FactSage calculations depict the FCC $\mathrm{Al}$ and $\mathrm{Cu}$ crystals in solid solution within the $\alpha$-Al grains. The growth of $\alpha-\mathrm{Al}$ grains is rapid from $650-610{ }^{\circ} \mathrm{C}$ and then continues steadily until the solidus temperature is reached at $\sim 555{ }^{\circ} \mathrm{C}$. As the temperature decreases, $\mathrm{Al}_{2} \mathrm{Cu}$ begins to precipitate at $\sim 530{ }^{\circ} \mathrm{C}$. The amount of $\mathrm{Al}_{2} \mathrm{Cu}$ continuously increases as the temperature decreases, while the amount of $\mathrm{Al}$ and $\mathrm{Cu}$ in solid solution decreases. At the final temperature of the calculation (i.e. $420{ }^{\circ} \mathrm{C}$ ), the estimated amounts of $\mathrm{Al}_{2} \mathrm{Cu}$ and $\alpha-\mathrm{Al}$ solid solution are $\sim 6 \mathrm{wt} \%$ and $94 \mathrm{wt} \%$, respectively. 
Construction of a tie line and application of the lever rule at $420{ }^{\circ} \mathrm{C}$ for the binary $\mathrm{Al}-5 \mathrm{wt} \% \mathrm{Cu}$ alloy on the $\mathrm{Al}-\mathrm{Cu}$ phase diagram (Figure 4-7) results in $\sim 6 \mathrm{wt} \%$ of $\mathrm{Al}_{2} \mathrm{Cu}$ and $\sim 94 \mathrm{wt} \%$ of $\alpha-\mathrm{Al}$ solid solution.

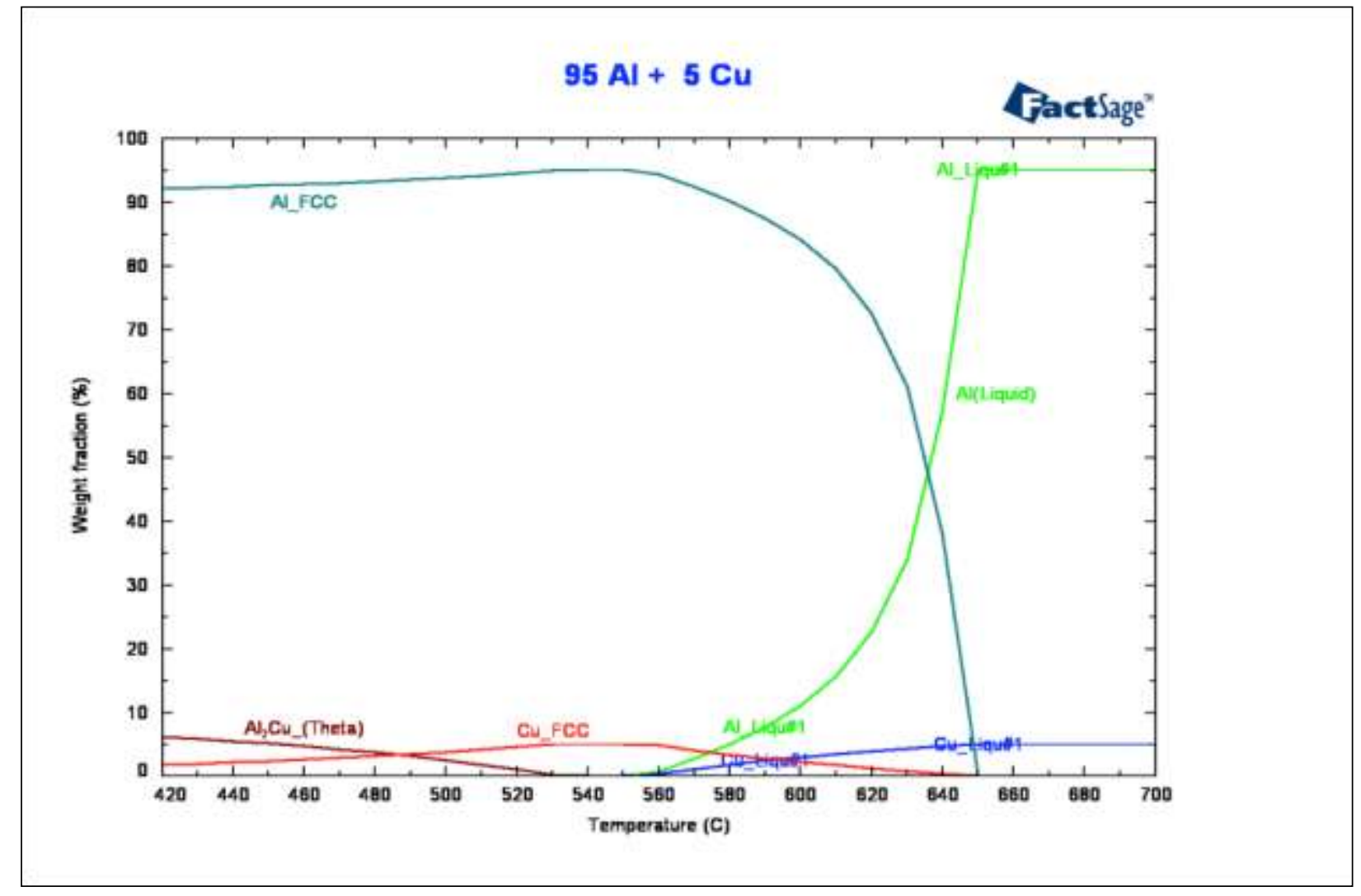

Figure 5-12. FactSage calculation of Al-5 wt\% $\mathrm{Cu}$ alloy for equilibrium solidification.

FactSage simulation was also carried out for the non-equilibrium solidification mode. The calculations using Scheil approach are shown in Figure 5-13. The results are presented for the temperature interval of $700-460{ }^{\circ} \mathrm{C}$. It can be seen from the plot (composition vs. temperature) that the nucleating temperature for the $\alpha$-Al solid solution crystals (i.e. liquidus temperature) is calculated at $\sim 646{ }^{\circ} \mathrm{C}$. The growth trend of $\alpha$-Al solid solution is in agreement to that of the equilibrium calculations (Figure 5-12), as rapid growth is first observed between the temperature interval of $646-610^{\circ} \mathrm{C}$ followed by a more steady growth until solidus. In this case, the solidus temperature is calculated at $\sim 548{ }^{\circ} \mathrm{C}$, in conjunction with the nucleation of the $\mathrm{Al}_{2} \mathrm{Cu}$ intermetallic. This temperature of $\mathrm{Al}_{2} \mathrm{Cu}$ nucleation is in agreement with the eutectic temperature according to the Al-Cu phase diagram (Figure 4-7). The use of the Scheil approach does not 
enable calculations beyond the precipitation of $\mathrm{Al}_{2} \mathrm{Cu}$. Thus the amount of $\mathrm{Al}_{2} \mathrm{Cu}$ could not be determined. However, the amount of $\alpha$-Al solid solution is estimated at $\sim 93 \mathrm{wt} \%$ at the end of solidification, also in good agreement with the equilibrium calculations.

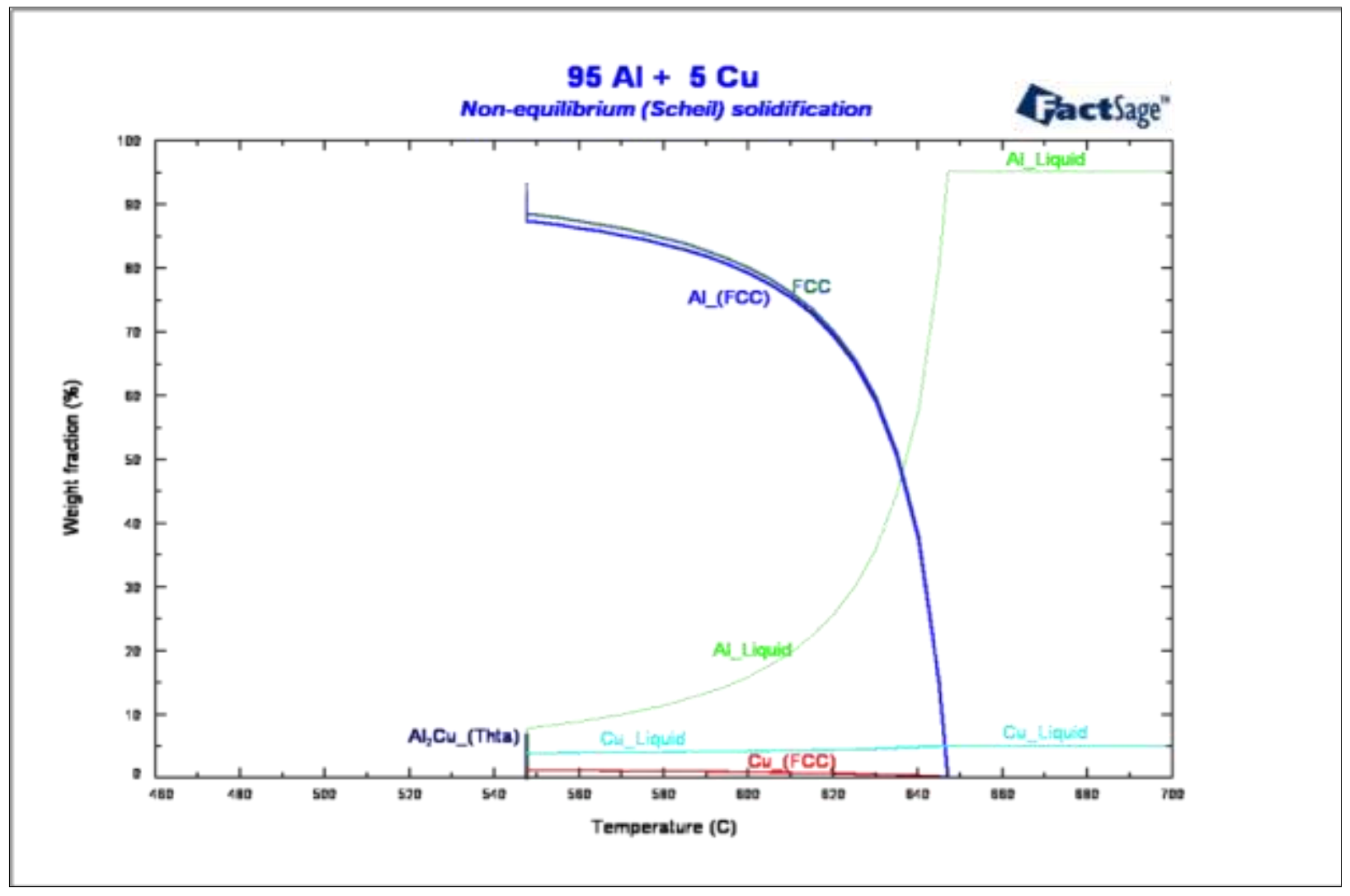

Figure 5-13. FactSage calculation of Al-5 wt \% $\mathrm{Cu}$ alloy for non-equilibrium solidification.

FactSage calculations were carried out as an approximation of the solidification process of the Al-5 wt\% $\mathrm{Cu}$ alloy. These calculations provided the basis for in situ neutron diffraction experiments, particularly in terms of temperature ranges and rates of phase evolution. The results of in situ neutron diffraction analysis are discussed in the following section.

\subsubsection{Growth of Primary Aluminum Phase}

The growth of the $\alpha-\mathrm{Al}$ solid solution phase for the three Al-5 wt $\% \mathrm{Cu}$ samples is presented in this section. A comparison of $\alpha-\mathrm{Al}$ solid solution grain growth between the three conditions is discussed with relation to the grain morphology of each alloy condition. 


\subsubsection{Grain Morphology of Samples}

The grain morphology of the binary Al-5 wt\% Cu samples was analyzed to confirm the effect of grain refinement with addition of Ti. The results are presented in Figures 5-14 and 5-15 for both the as-cast (i.e. prior to neutron diffraction) and post-neutron diffraction conditions, respectively. Figure 5-14 confirms the findings of Sections 4.3.2 and 5.3.1 as additions of Ti resulted in a significant decrease in grain size and a transformation of grain morphology from a coarse dendritic structure to a more globular one.
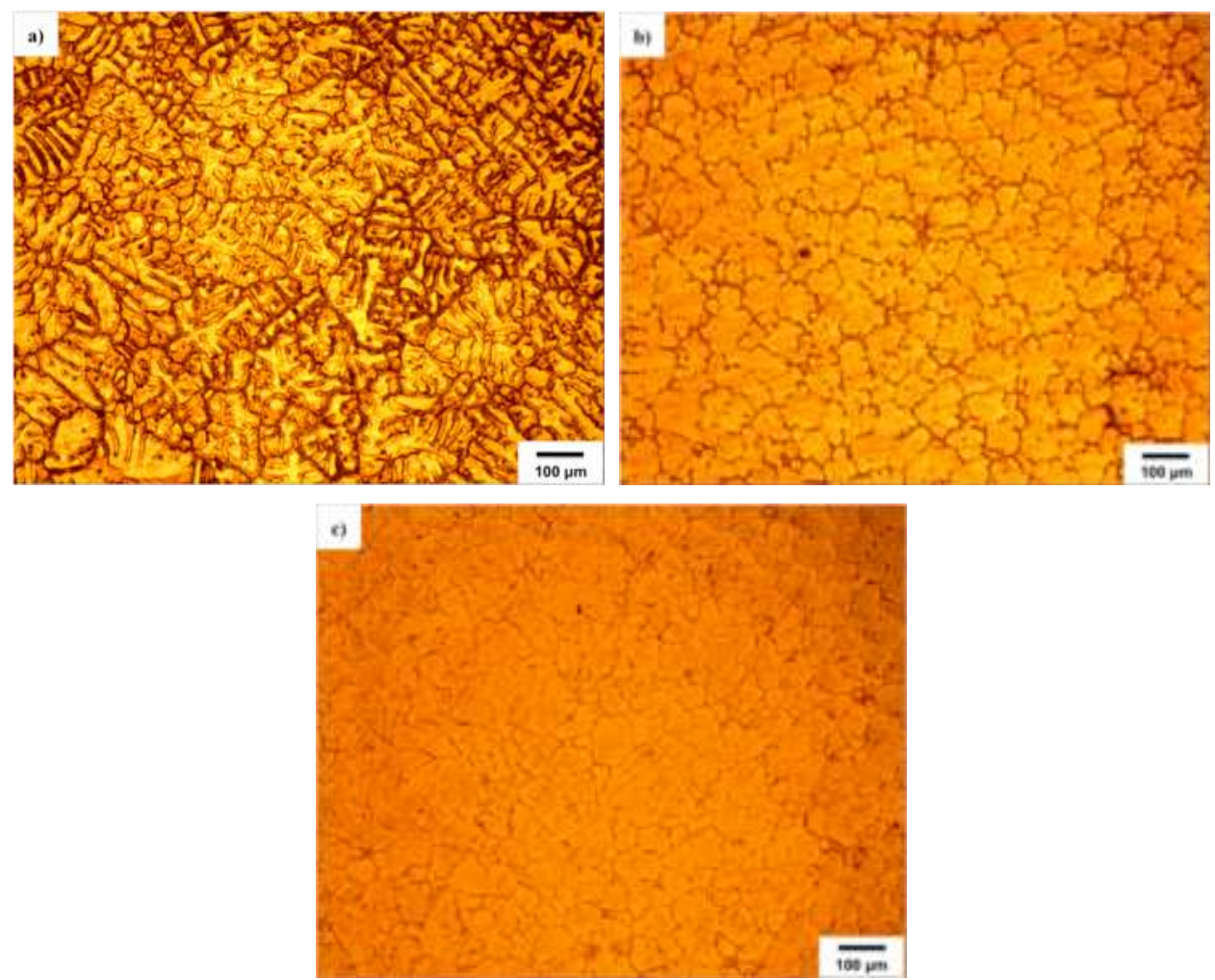

Figure 5-14. Grain morphology of a) unrefined; b) $0.02 \mathrm{wt} \% \mathrm{Ti}$ and c) $0.05 \mathrm{wt} \% \mathrm{Ti}$ binary alloy prior to neutron diffraction solidification analysis. 

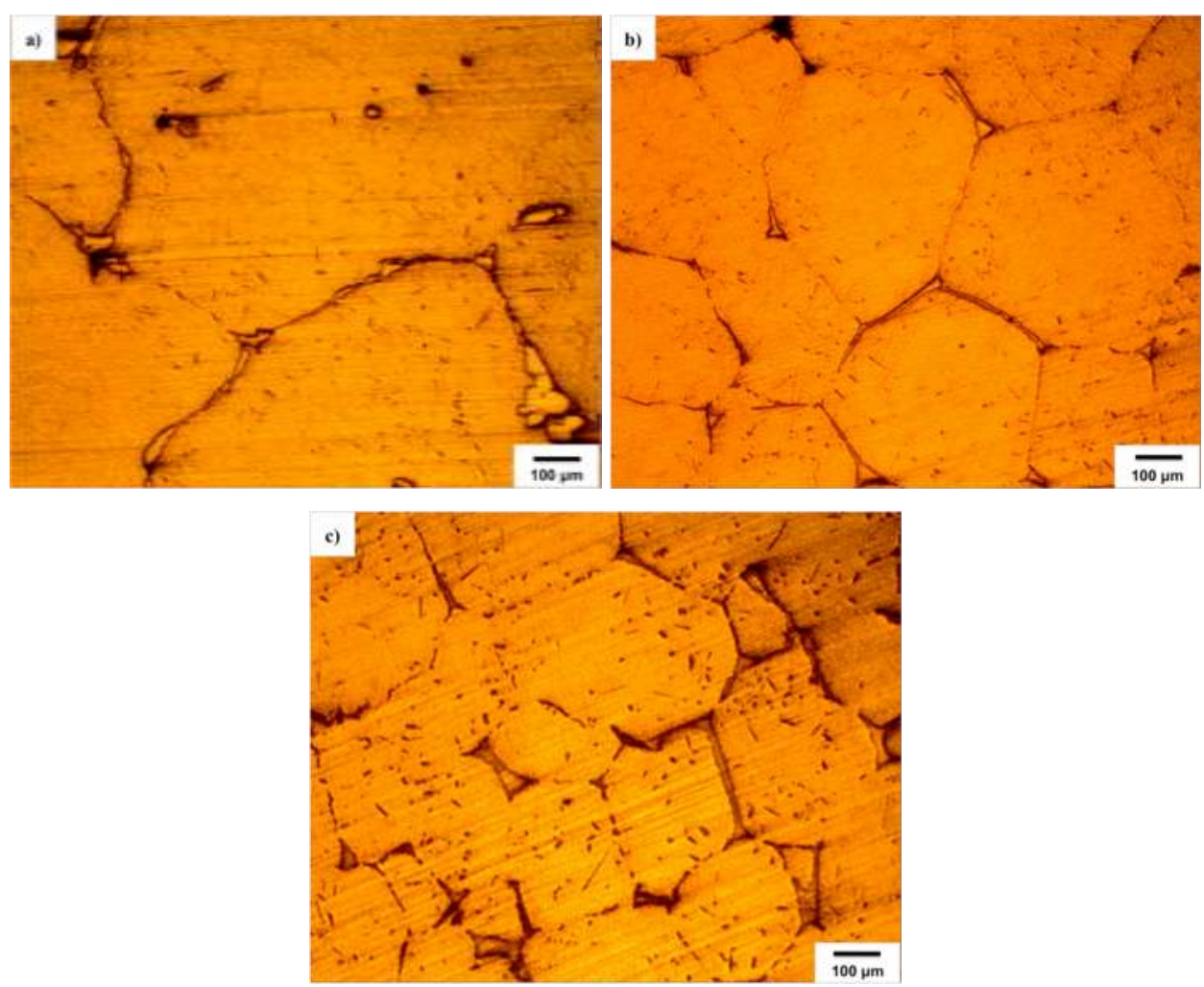

Figure 5-15. Grain morphology of a) unrefined; b) $0.02 \mathrm{wt} \%$ Ti and c) $0.05 \mathrm{wt} \%$ Ti binary alloy following neutron diffraction solidification analysis.

Solidification analysis via neutron diffraction was seen to increase the overall grain size of the samples, as shown in Figure 5-15. This was likely due to the slower cooling rates experienced by the samples during neutron diffraction (in comparison to those during permanent mold casting). Nevertheless, grain refinement with additions of Ti was confirmed in these samples as well, as the coarse dendrites seen in the unrefined alloy (Figure 5-15a) were transformed to finer equiaxed grains for the refined alloys (Figures 5-15b and c).

\subsubsection{Primary Aluminum Peak Evolution}

Two solid Al-rich peaks (i.e. (111) and (200)) were visible within the angular range of diffraction angles (i.e. 37 to 117 degrees). A representative plot of the peak evolution of the Al-rich phase for the (111) reflection (denoted as $\mathrm{Al}_{(111)}$ from hereon) is shown in Figure 5-16 for the solidification interval between $650{ }^{\circ} \mathrm{C}$ and $530{ }^{\circ} \mathrm{C}$. The remaining plots of peak evolution for the additional (200) reflection are shown in Appendix 6. The $\mathrm{Cu}$ in the $\mathrm{Al}-5 \mathrm{wt} \% \mathrm{Cu}$ binary alloy 
was present in solid solution within $\alpha-\mathrm{Al}$ grains and therefore was part of the $\alpha$-Al solid solution pattern.

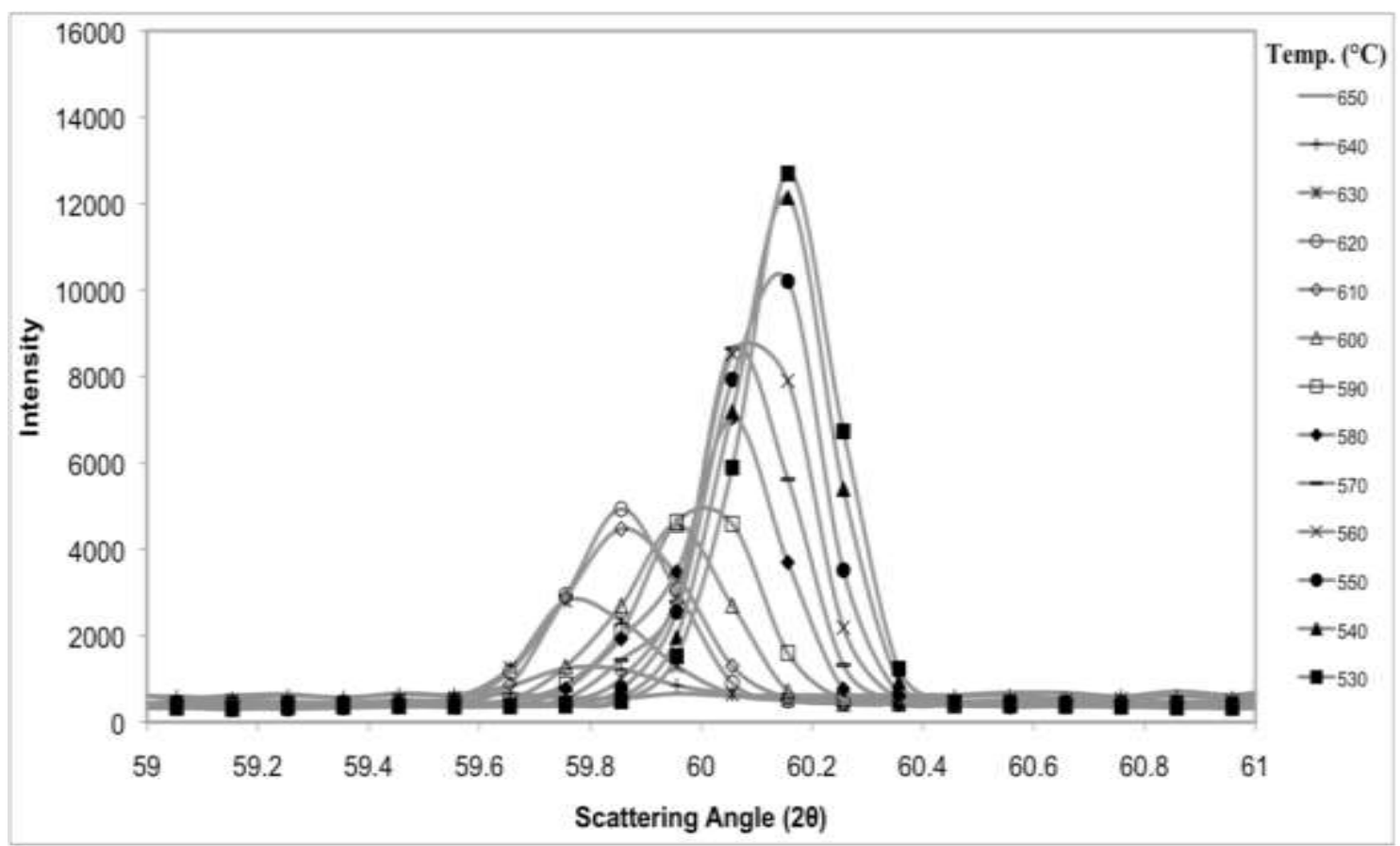

Figure 5-16. Peak evolution of the $\alpha-\mathrm{Al}_{(111)}$ phase.

Initially, at temperatures where solid crystals were beginning to evolve, a high 'background' was observed as a result of diffuse scattering. As the temperature of the alloy decreased however, the 'background' was lowered and peaks began to develop, as elastic scattering of the developing solid commenced (Figure 5-16). Generally, as the alloy temperature decreased further, the area beneath the peaks was found to increase. Also, the angular position of the peaks shifted, corresponding to thermal contraction of the solid alloy (i.e. a reduction in lattice spacing reflected by the shift in Bragg's peak position) [81]. Minor lattice spacing contraction may have also resulted from the enrichment of $\mathrm{Al}$ solid solution with $\mathrm{Cu}$, but such shifts are minimal in comparison to those due to thermal contraction $[82,83]$. Integration of the peak's normalized intensity over the angular range covering the peak's width was carried out. This enabled the retrieval of relative solid fraction (or fraction of solid phase) for the selected temperatures within the solidification interval of the alloy. The results are presented in the following section. 


\subsubsection{Primary Aluminum Phase Evolution}

The phase evolution of $\alpha-\mathrm{Al}_{(111)}$ solid solution is shown in Figure 5-17 for the unrefined and refined Al-5 wt $\% \mathrm{Cu}$ binary alloys. In the case of the unrefined alloy, the evolution of $\alpha$-Al solid solution was first detected at $650{ }^{\circ} \mathrm{C}$. As the temperature decreased, the $\alpha$-Al evolved rapidly, as at $630{ }^{\circ} \mathrm{C}$, the fraction of solid phase was $\sim 0.8$. Beyond $630{ }^{\circ} \mathrm{C}$, the growth in $\alpha$ - $\mathrm{Al}$ dendrites was more gradual and finally reached $100 \%$ solid at $530{ }^{\circ} \mathrm{C}$. Such growth of the $\alpha$-Al solid solution was in close agreement to the FactSage calculations (Figure 5-12 and 5-13).

It is difficult to obtain the exact solidus temperature of the $\mathrm{Al}-5 \mathrm{wt} \% \mathrm{Cu}$ alloy, as the neutron diffraction data was detected in increments of temperature over a solidification range. However, the integrated diffraction intensity obtained at $530{ }^{\circ} \mathrm{C}$ remained the same as the temperature was decreased further. As a result, this suggests that at this temperature, the $\alpha$-Al was completely developed for this alloy.

The growth of $\alpha-\mathrm{Al}$ in the $0.02 \mathrm{wt} \% \mathrm{Ti}$ and $0.05 \mathrm{wt} \% \mathrm{Ti}$ alloys was significantly more gradual with respect to that in the unrefined alloy. The first evidence of $\alpha$-Al solid solution nucleation $\left(<1 \%\right.$ ) was detected at $650{ }^{\circ} \mathrm{C}$ for both alloys. The $\alpha-\mathrm{Al}$ in the $0.05 \mathrm{wt} \% \mathrm{Ti}$ alloy then continued to evolve to $\sim 0.38$ at $620{ }^{\circ} \mathrm{C}$, where it then became almost stagnant until $590{ }^{\circ} \mathrm{C}$. In the case of the $0.02 \mathrm{wt} \% \mathrm{Ti}$ alloy, the $\alpha-\mathrm{Al}$ increased rapidly (with respect to the $0.05 \mathrm{wt} \% \mathrm{Ti}$ alloy) between the temperatures of $620{ }^{\circ} \mathrm{C}$ and $610{ }^{\circ} \mathrm{C}$. At this point, the growth of $\alpha$-Al became stagnant for this alloy as well until $580{ }^{\circ} \mathrm{C}$. The regions of stagnant growth in both alloys were likely attributed to the onset of dendrite (or grain) coherency. Once the $\alpha$-Al grains began to impinge on one another, the growth likely stalled, as evidenced by the curves in Figure 5-17. A similar result was also seen for the unrefined alloy at $630{ }^{\circ} \mathrm{C}$, which confirms the delay in coherency temperature for the refined alloys observed in Section 4.4.3. Following coherency, the $\alpha$-Al grains in both refined alloys continued to grow almost linearly to $100 \%$ solid at $530{ }^{\circ} \mathrm{C}$. As was

the case for the unrefined alloy, the exact solidus temperature could not be detected for the refined alloys. 
The variation observed in the development of $\alpha$-Al solid solution for the unrefined and refined alloys was directly attributed to their differences in alloy grain morphology. The unrefined alloy microstructure was characterized with coarse dendrites that likely nucleated from the cooler mold wall and propagated rapidly towards the warmer centre of the casting. Such rapid growth of the dendrites was in agreement with the development of $\alpha$-Al solid solution illustrated in Figure 5-17. In the case of the refined alloys, however, it was not required for nucleation to originate from the mold wall, as Ti-based nucleating particles were added to the melt via the Al-5Ti-1B master alloy. These nucleating particles subsequently promoted heterogeneous nucleation, which thereby enabled uniform grain growth resulting in equiaxed grain morphologies. This is confirmed by the quasi-linear development of $\alpha$-Al solid solution in Figure 5-17 for both refined alloys. The slight differences observed between the two refined alloys could also be attributed to their respective grain size and morphology. The slightly larger and less globular grains of the $0.02 \mathrm{wt} \% \mathrm{Ti}$ alloy were likely the result of a faster initial growth of $\alpha$-Al due to the presence of fewer nucleating particles.

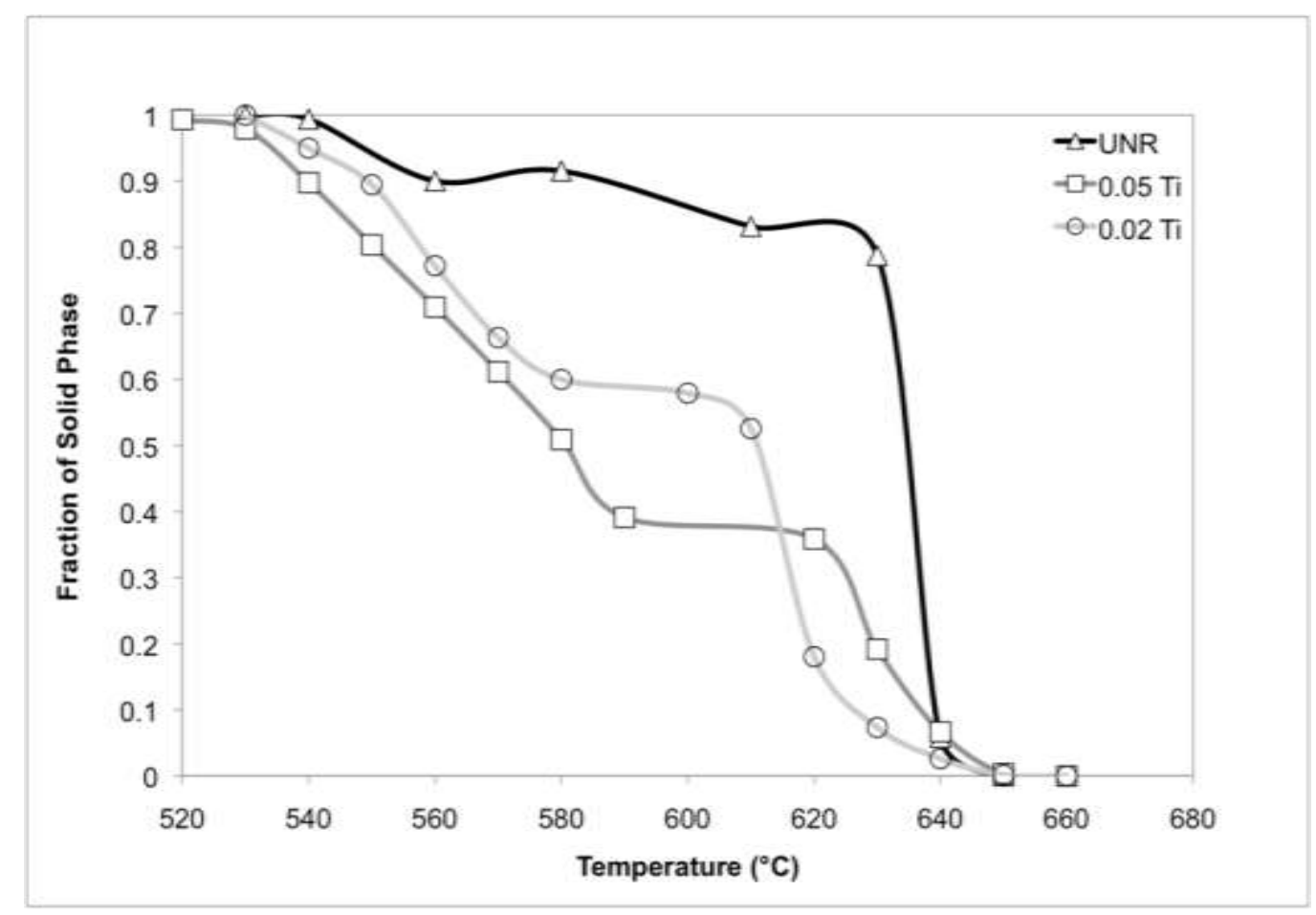

Figure 5-17. Phase evolution of $\alpha-\mathrm{Al}_{(111)}$ solid solution in the unrefined (UNR), $0.02 \mathrm{wt} \% \mathrm{Ti}$ and 0.05 wt \% Ti binary Al-5 wt\% Cu alloys. 
The growth of $\alpha-\mathrm{Al}$ for the (200) plane (i.e. $\mathrm{Al}_{(200)}$ ) is presented in Appendix 6 (Figure A.6-5) for only the refined alloys (i.e. $0.02 \mathrm{wt} \% \mathrm{Ti}$ and $0.05 \mathrm{wt} \% \mathrm{Ti}$ ), since the $\mathrm{Al}_{(200)}$ peak was not detected during neutron diffraction for the unrefined alloy. Similar profiles were seen for the growth of $\mathrm{Al}_{(200)}$ for both alloys. The $\alpha$ - $\mathrm{Al}$ solid solution was first detected $(\sim 12 \%)$ at $650{ }^{\circ} \mathrm{C}$ and developed steadily until $620^{\circ} \mathrm{C}$. At this temperature, the onset of grain coherency was likely obtained, as the development of $\alpha$-Al was seen to stall in both alloys until $600{ }^{\circ} \mathrm{C}$. Beyond $600{ }^{\circ} \mathrm{C}$, the $\alpha-\mathrm{Al}_{(200)}$ continued to increase to $100 \%$ solid at $530{ }^{\circ} \mathrm{C}$ for both alloys. The region of stagnant growth in the alloys showed close agreement to that of the $\alpha-\mathrm{Al}_{(111)}$ in Figure 5-17.

\subsubsection{Growth of Intermetallic $\mathrm{Al}_{2} \mathrm{Cu}$}

\subsubsection{Intermetallic $\mathrm{Al}_{2} \mathrm{Cu}$ Peak Evolution}

Figure 5-18 illustrates the peak evolution of the $\mathrm{Al}_{2} \mathrm{Cu}$ intermetallic phase for the (310) reflection (hereafter denoted as $\mathrm{Al}_{2} \mathrm{Cu}_{(310)}$ ). The (310) reflection was the only reflection for which the $\mathrm{Al}_{2} \mathrm{Cu}$ was detected for the three alloys. The peak evolution for $\mathrm{Al}_{2} \mathrm{Cu}_{(310)}$ is shown between $560{ }^{\circ} \mathrm{C}$ and $440{ }^{\circ} \mathrm{C}$. The peak evolution of $\mathrm{Al}_{2} \mathrm{Cu}_{(310)}$ for the remaining two samples is given in Appendix 6 (Figures A.6-6 and A.6-7). The peak-to-background ratio was significantly lower for the $\mathrm{Al}_{2} \mathrm{Cu}$ phase in comparison to that of $\alpha-\mathrm{Al}$ (Figure 5-16) due to its lower concentration in the

alloy. Inspection of Figure 5-18 also reveals both the increase in area beneath the peaks with decrease in temperature, as well as the shift in angular position of the peaks due to thermal contraction of the solid. Integration of the peak's normalized intensity over the angular range covering the peak's width was carried out. This enabled calculation of the amount of intermetallic $\mathrm{Al}_{2} \mathrm{Cu}$ for the selected temperature range of neutron diffraction analysis (i.e. $\left.560-440{ }^{\circ} \mathrm{C}\right)$. The results are presented in the following section. 


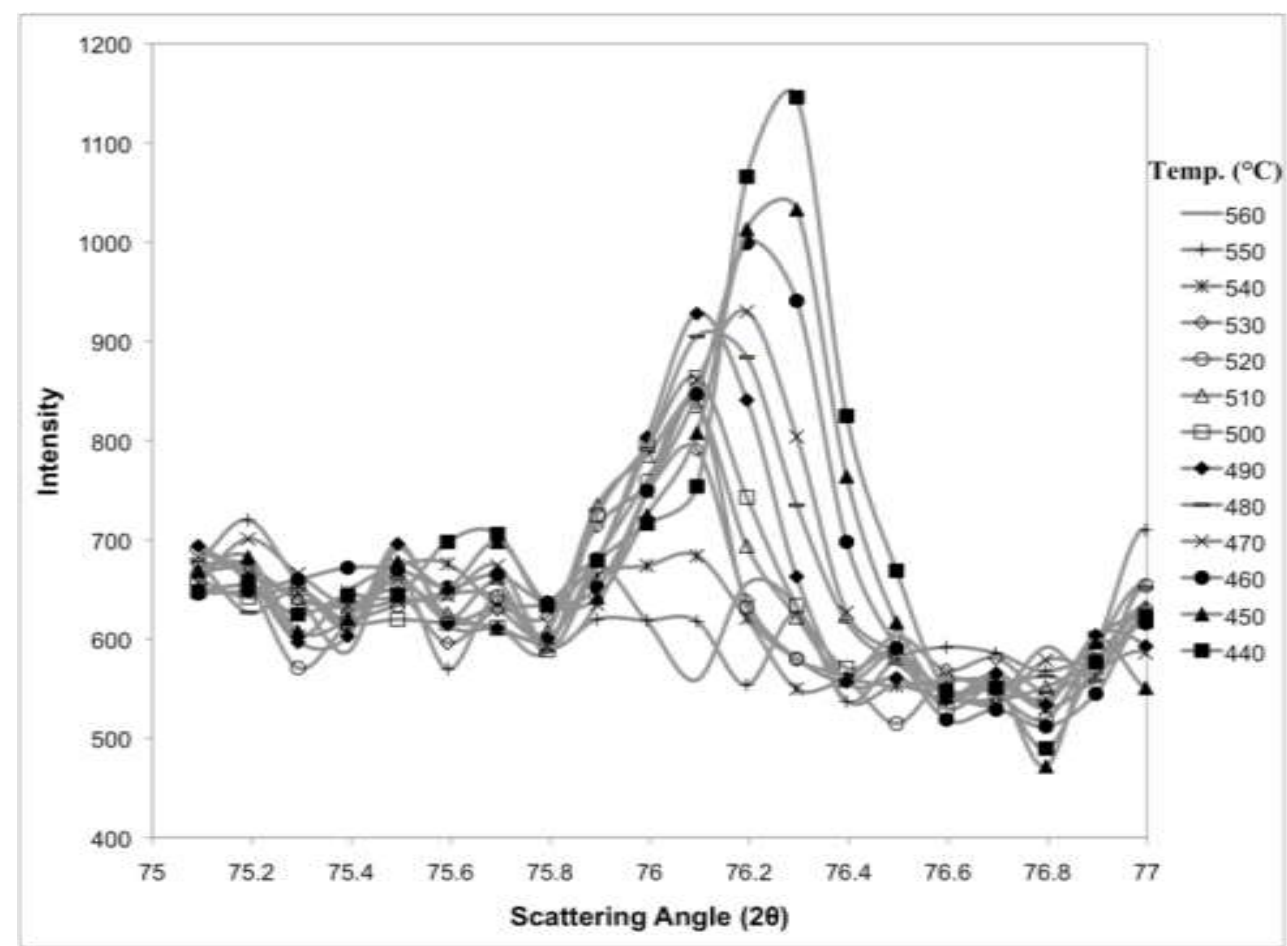

Figure 5-18. Peak evolution of the $\mathrm{Al}_{2} \mathrm{Cu}_{(310)}$ intermetallic phase for the $0.05 \mathrm{wt} \% \mathrm{Ti}$ alloy.

\subsubsection{Intermetallic $\mathrm{Al}_{2} \mathrm{Cu}$ Phase Evolution}

The development of intermetallic $\mathrm{Al}_{2} \mathrm{Cu}$ for the three binary alloy conditions is illustrated in Figure 5-19. The amount of $\mathrm{Al}_{2} \mathrm{Cu}$ is presented in wt\% as a function of temperature. The final temperature of analysis was $440{ }^{\circ} \mathrm{C}$ for the unrefined and $0.02 \mathrm{wt} \% \mathrm{Ti}$ alloy, while for the $0.05 \mathrm{wt} \% \mathrm{Ti}$ alloy, neutron diffraction analysis was carried out to $420{ }^{\circ} \mathrm{C}$. The amount of $\mathrm{Al}_{2} \mathrm{Cu}$ at $420{ }^{\circ} \mathrm{C}$ was estimated at $6 \mathrm{wt} \%$, as determined from both the $\mathrm{Al}-\mathrm{Cu}$ phase diagram (Figure 4-7) and FactSage simulation (Figure 5-12). The actual volume fraction of $\mathrm{Al}_{2} \mathrm{Cu}$ with respect to temperature was not determined in this research.

The addition of $\mathrm{Ti}$ was found to have an effect on the precipitation temperature of $\mathrm{Al}_{2} \mathrm{Cu}$, as the first detection of this phase was at $\sim 540{ }^{\circ} \mathrm{C}$ for the unrefined alloy and at $\sim 550{ }^{\circ} \mathrm{C}$ for the refined alloys. These temperatures were similar to those predicted by the non-equilibrium solidification FactSage calculations (i.e. $548^{\circ} \mathrm{C}$ ). As the temperature decreased, the growth of $\mathrm{Al}_{2} \mathrm{Cu}$ followed similar profiles for the three alloys. Thus, grain refinement was not seen to influence the actual growth of $\mathrm{Al}_{2} \mathrm{Cu}$ despite affecting its nucleation temperature. 


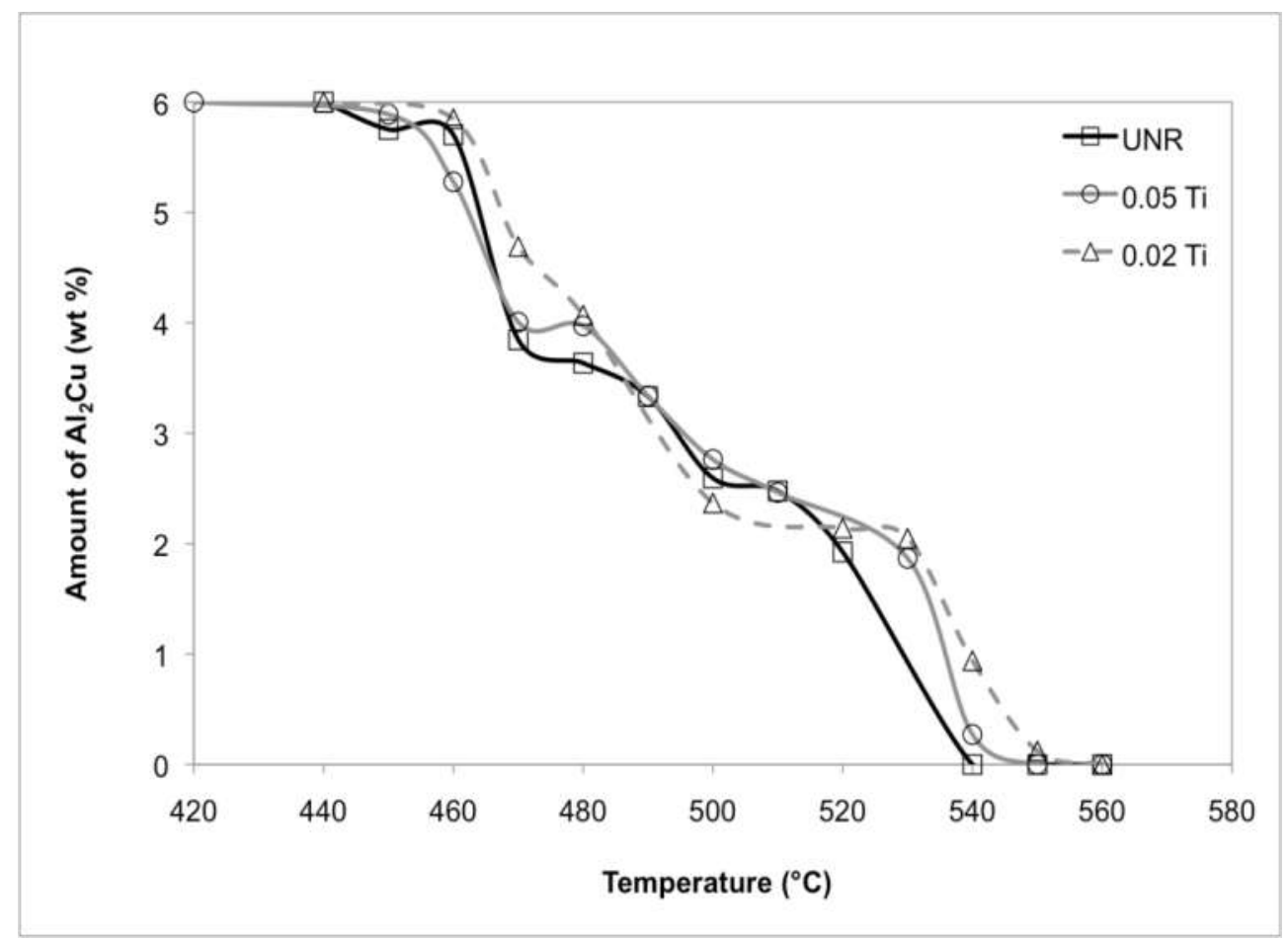

Figure 5-19. Phase evolution of intermetallic $\mathrm{Al}_{2} \mathrm{Cu}_{(310)}$ in the unrefined (UNR), 0.02 wt \% Ti and 0.05 wt \% Ti binary Al-5 wt\% Cu alloys.

Conventional thermal analysis techniques [84] and in situ neutron diffraction strain analysis [85] were used in past research to determine the temperature of nucleation for $\mathrm{Al}_{2} \mathrm{Cu}$ in $\mathrm{Al}-\mathrm{Cu}$ alloys. This research however, was the first to establish the actual growth profile of the intermetallic phase. The growth of $\mathrm{Al}_{2} \mathrm{Cu}$ is an important factor in advancing the understanding of the role of microstructure on hot tearing in B206. The following section discusses the significance of these findings with relation to the formation of hot tears in B206.

\subsubsection{The Role of Microstructure Development on Hot Tearing in B206}

\subsubsection{Onset Solid Fraction of Hot Tearing in B206}

The onset temperatures of hot tearing recorded from Figure 5-2 were used in conjunction with the phase evolution of $\alpha$-Al solid solution in Figure 5-17 to estimate the onset solid fraction of hot tearing for the unrefined B206 alloys. The respective onset solid fractions for the $250{ }^{\circ} \mathrm{C}$, $325^{\circ} \mathrm{C}$ and $400{ }^{\circ} \mathrm{C}$ mold temperatures were $0.85,0.87$ and 0.81 . The secondary crack observed 
for the $400{ }^{\circ} \mathrm{C}$ mold temperature was found to initiate at a solid fraction of 0.85 . Thus, mold temperature was not found to have a significant effect on the onset solid fraction of hot tearing in B206. Similar results were observed in a previous study on magnesium alloys [46]. Past studies as summarized in the review by Eskin et al. [6] have suggested that hot tearing generally occurs in the solid fraction range of 0.85 to 0.95 . Further, investigations by Cao et al. [53] showed that hot tears can initiate at solid fractions as low as 0.78 . Therefore, the results in this study are consistent with literature.

In previous studies $[31,32,38,46,53]$, the solid fractions corresponding to hot tear initiation were determined via thermodynamic calculations using equilibrium or Scheil's model. Such calculations are not reliable, as they are based on assumptions (e.g. no solid-state diffusion, equilibrium at solid-liquid interface) and therefore are not representative of actual solidification. Further, these models are not capable of determining the effect of grain refinement on solid fraction development. The effect of grain refinement was made possible in this study through the use of neutron diffraction, as illustrated in Figure 5-17. These results were used to gain an advanced understanding of the role of microstructure development on hot tearing in B206.

\subsubsection{Development of Microstructure in B206}

The in situ phase evolution of $\alpha-\mathrm{Al}$ solid solution (Figure 5-17) and $\mathrm{Al}_{2} \mathrm{Cu}$ (Figure 5-19) was a unique contribution to this research that can be used to improve the understanding of the mechanisms of hot tearing in $\mathrm{B} 206$. The intermetallic $\mathrm{Al}_{2} \mathrm{Cu}$ phase was seen to nucleate well after the initiation of hot tears in B206 (Figure 5-2). Further, the nucleation of $\mathrm{Al}_{2} \mathrm{Cu}$ did not commence until nearly $100 \%$ solid $\alpha$-Al was formed in the unrefined alloy, as confirmed by the combined growth profiles of $\alpha-\mathrm{Al}$ solid solution and $\mathrm{Al}_{2} \mathrm{Cu}$ in Figure 5-20. Thus, a continuous rigid network of $\alpha$-Al dendrites was fully developed in the unrefined alloy, thereby limiting the $\mathrm{Al}_{2} \mathrm{Cu}$ to small interdendritic regions. Further, this likely had an impact on the morphology of $\mathrm{Al}_{2} \mathrm{Cu}$. The intermetallic $\mathrm{Al}_{2} \mathrm{Cu}$ phase is usually present as two different morphologies: blocky and eutectic [86]. The blocky morphology is a result of solid-state precipitation while the eutectic morphology precipitates via the eutectic reaction. In the case of the unrefined alloy, since $\mathrm{Al}_{2} \mathrm{Cu}$ precipitated after the $\alpha-\mathrm{Al}$ was nearly complete, this suggests that the majority of 
$\mathrm{Al}_{2} \mathrm{Cu}$ development was through solid-state precipitation, resulting in mostly blocky $\mathrm{Al}_{2} \mathrm{Cu}$ in the alloy. The alloy microstructure was examined using SEM and the resulting micrographs in Figure 5-21 demonstrate the significant presence of blocky $\mathrm{Al}_{2} \mathrm{Cu}$. The limited presence of eutectic $\mathrm{Al}_{2} \mathrm{Cu}$ likely had a significant influence on liquid feeding in the unrefined casting.

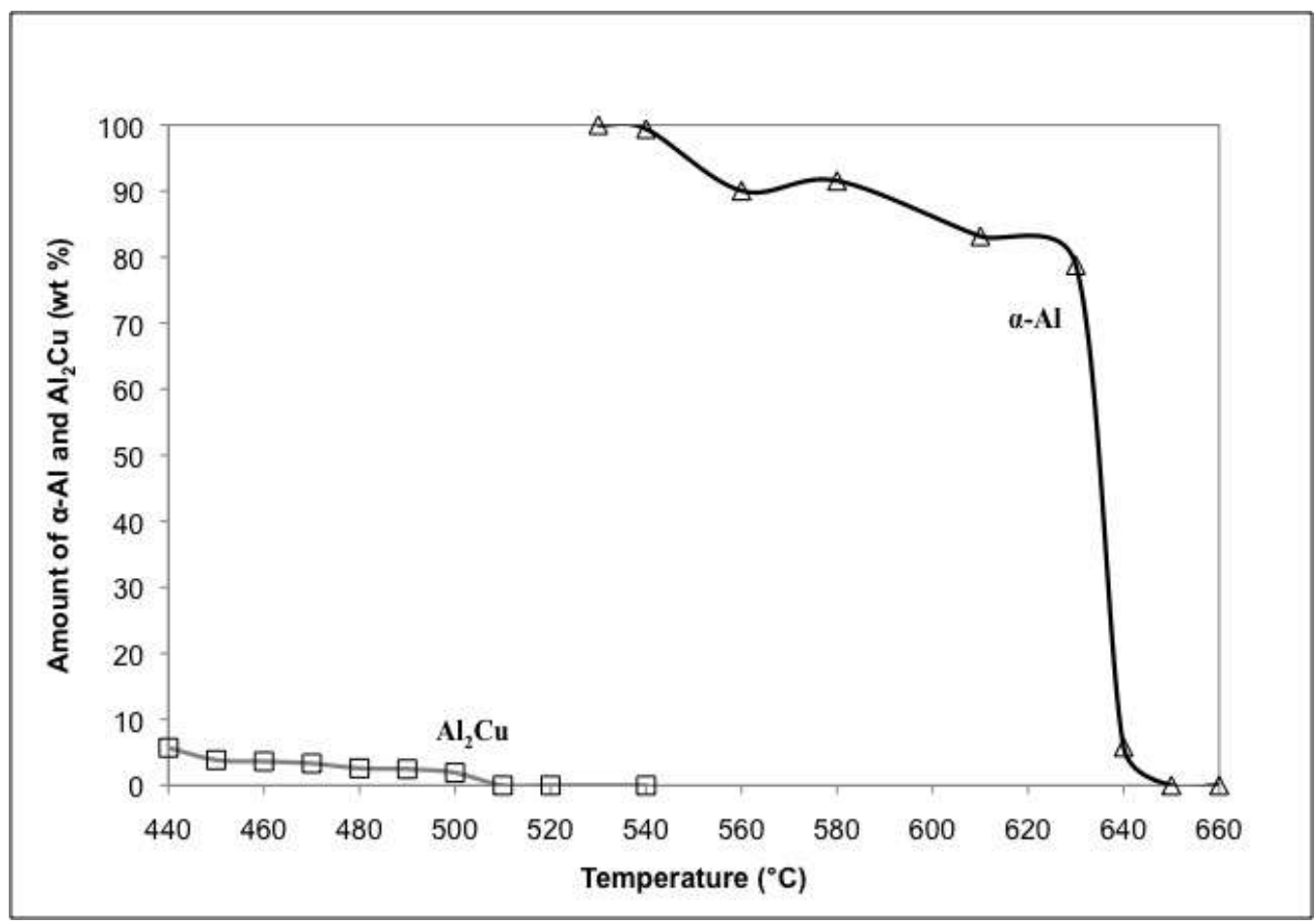

Figure 5-20. Combined growth profiles of $\alpha-\mathrm{Al}$ and $\mathrm{Al}_{2} \mathrm{Cu}$ in the unrefined alloy.

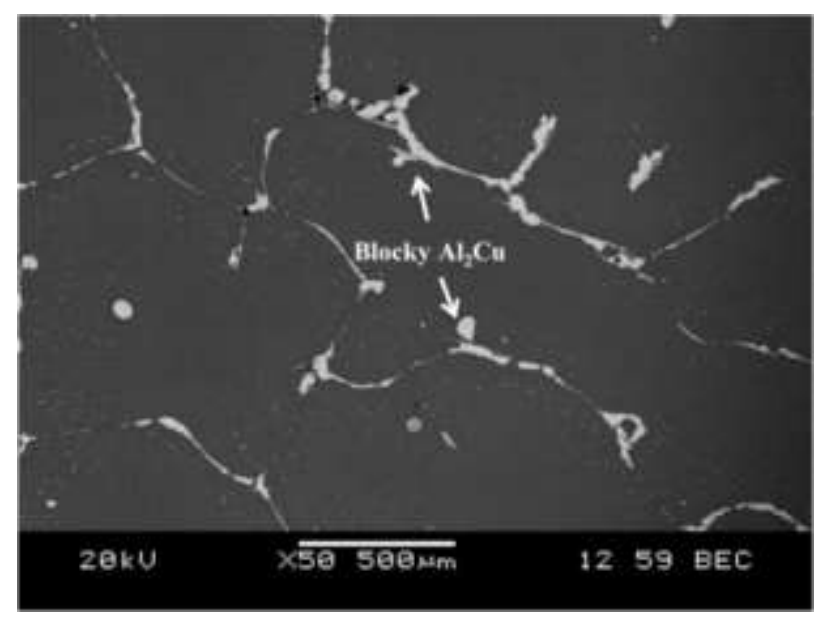

a)

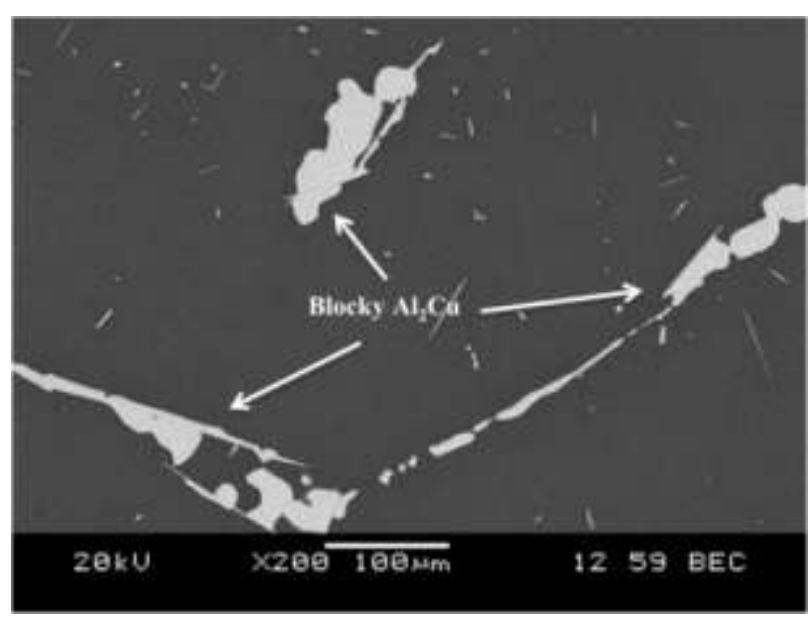

b)

Figure 5-21. The presence of blocky $\mathrm{Al}_{2} \mathrm{Cu}$ in the unrefined alloy at a) 50x and b) 200x magnification. 
The significant presence of blocky $\mathrm{Al}_{2} \mathrm{Cu}$ in the unrefined alloy suggests that the majority of $\mathrm{Al}_{2} \mathrm{Cu}$ formed via solid-state precipitation at interdendritic regions. Thus, a limited amount of liquid of eutectic composition (denoted eutectic liquid hereafter) (present during the eutectic reaction) was available to feed the casting at late stages of solidification. As a result, late stage feeding of eutectic liquid was not significant enough to accommodate the shrinkage of the dendritic network in the unrefined alloy, thereby resulting in many areas of porosity, as demonstrated in Figure 4-28 (Section 4.3.5). A similar occurrence took place during the development of hot tears, as insufficient amounts of eutectic liquid could not heal developed hot tears along interdendritic regions.

In the case of the refined alloys, the $\alpha$-Al solid solution was less developed (relative to the unrefined alloy) at the instant $\mathrm{Al}_{2} \mathrm{Cu}$ began to precipitate. The combined growth profiles of $\alpha-\mathrm{Al}$ and $\mathrm{Al}_{2} \mathrm{Cu}$ in Figures 5-22 and 5-23 show that the temperature of nucleation of $\mathrm{Al}_{2} \mathrm{Cu}$ was coincident with a minimum of $\sim 90 \%$ solid fraction of $\alpha$-Al solid solution in both refined alloys. Thus, there was a greater amount of eutectic liquid (at the instant of $\mathrm{Al}_{2} \mathrm{Cu}$ nucleation) present in the refined alloys, which underwent the eutectic reaction and thereby resulted in enhanced precipitation of eutectic $\mathrm{Al}_{2} \mathrm{Cu}$ (vs. blocky $\mathrm{Al}_{2} \mathrm{Cu}$ ). Scanning electron microscopy of the postneutron diffraction samples (from Figure 5-16) was carried out to confirm this. Representative micrographs for the $0.05 \mathrm{wt} \% \mathrm{Ti}$ alloy in Figure 5-24 illustrate the presence of both eutectic and blocky $\mathrm{Al}_{2} \mathrm{Cu}$. A similar result was also observed for the $0.02 \mathrm{wt} \% \mathrm{Ti}$ alloy.

The increased presence of eutectic $\mathrm{Al}_{2} \mathrm{Cu}$ likely improved feeding at late stages of solidification for the refined alloys and thus significantly enhanced their resistance to porosity and hot tearing. The overall feeding was facilitated for the refined alloys, since the $\alpha$-Al grains were not yet completely impinging on one another at the time of $\mathrm{Al}_{2} \mathrm{Cu}$ nucleation. This likely enabled eutectic liquid to readily flow through the intergranular regions of the developing grains, thereby enhancing the permeability of the solidifying casting at late stages of solidification. As a result, the refined castings were better capable of accommodating strain during solidification. Further, in conjunction with the improved distribution of $\mathrm{Al}_{2} \mathrm{Cu}$ observed in Figures 4-12, 4-13 and 5-10 (Sections 4.3.3 and 5.3.2), an overall enhanced and uniform feeding of liquid occurred in the 
refined alloys (in particular in the $0.05 \mathrm{wt} \% \mathrm{Ti}$ alloy), which in turn resulted in decreased porosity (Figure 4-27) and enhanced hot tearing resistance.

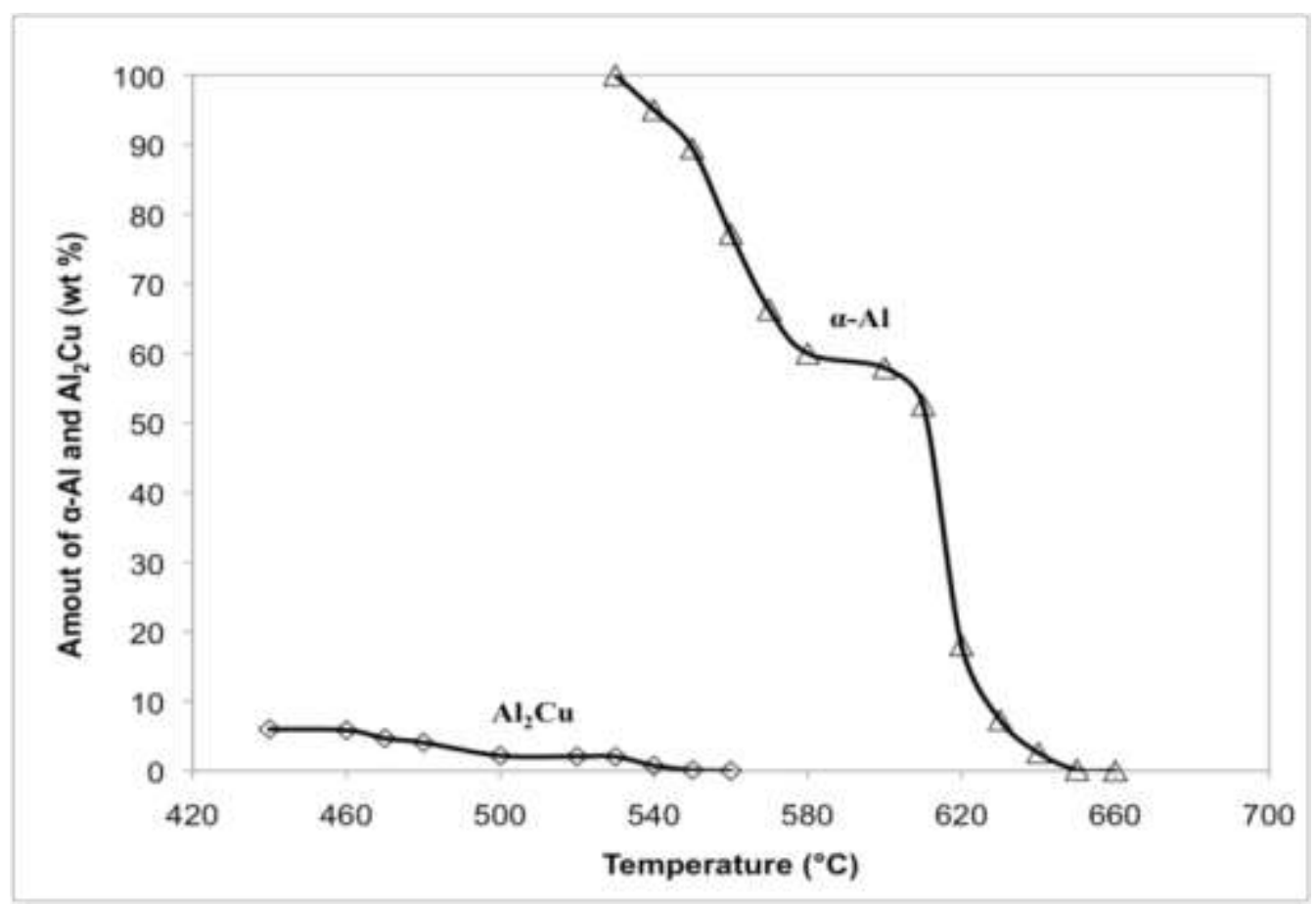

Figure 5-22. Combined growth profiles of $\alpha-\mathrm{Al}$ and $\mathrm{Al}_{2} \mathrm{Cu}$ in the 0.02 wt\% $\mathrm{Ti}$ alloy.

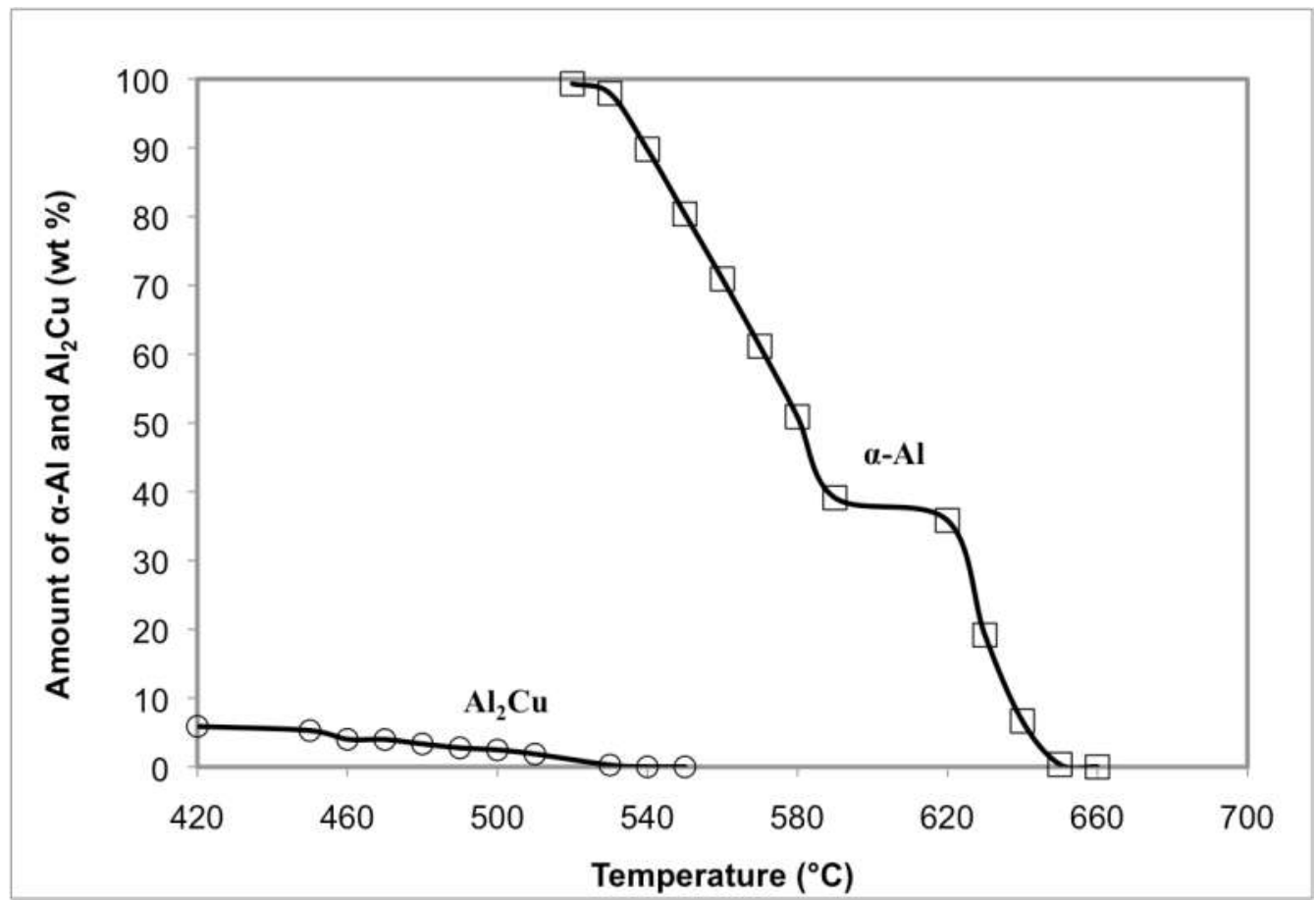

Figure 5-23. Combined growth profiles of $\alpha-\mathrm{Al}$ and $\mathrm{Al}_{2} \mathrm{Cu}$ in the $0.05 \mathrm{wt} \% \mathrm{Ti}$ alloy. 


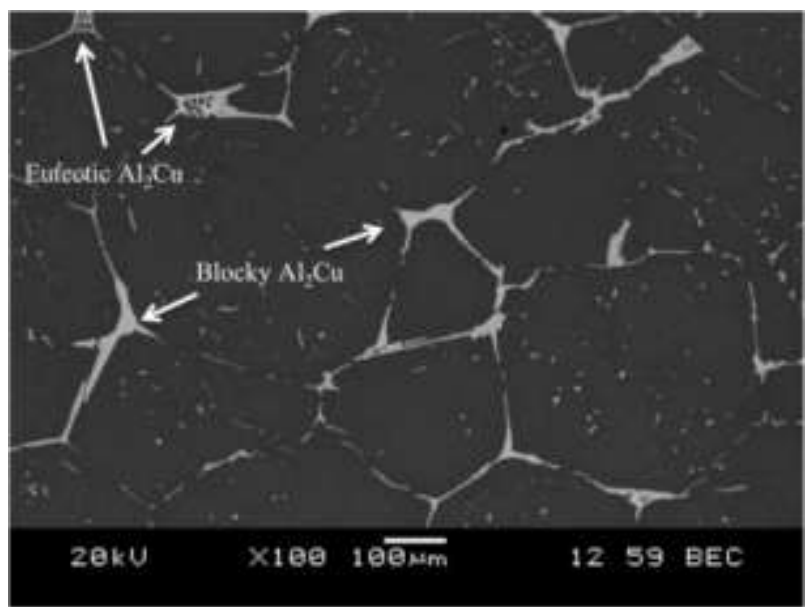

a)

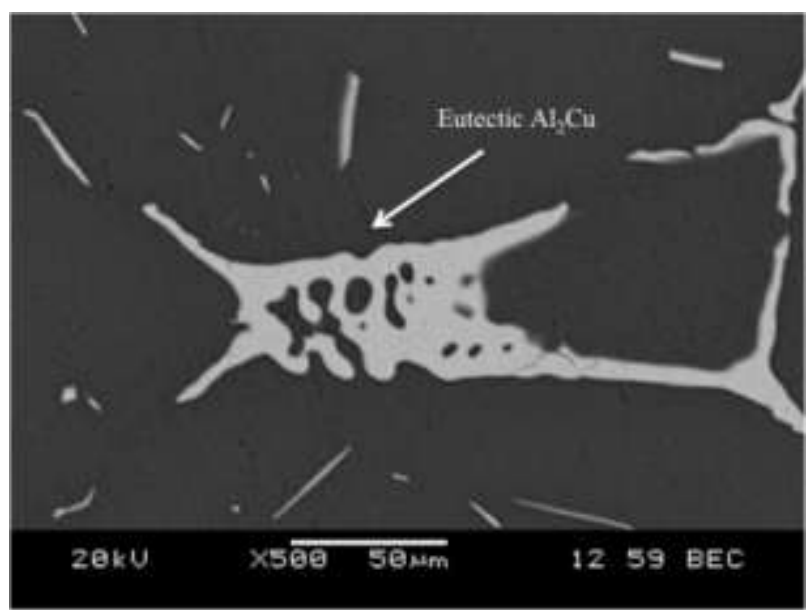

b)

Figure 5-24. The presence of blocky and eutectic $\mathrm{Al}_{2} \mathrm{Cu}$ in the $0.05 \mathrm{wt} \% \mathrm{Ti}$ alloy at a) $100 \mathrm{x}$ and b) 500x magnification.

\section{Section Summary}

In situ neutron diffraction solidification analysis provided an increased understanding of the development of microstructure in the unrefined and grain-refined binary Al-5 wt\% Cu alloys. The distinct solid phase evolution of $\alpha$-Al solid solution and intermetallic $\mathrm{Al}_{2} \mathrm{Cu}$ were successfully detected for each condition. The growth profiles of $\alpha$-Al solid solution showed good correlation with the grain morphology of the respective alloys. In the case of $\mathrm{Al}_{2} \mathrm{Cu}$, in situ neutron diffraction established that grain refinement resulted in an increase in the nucleating temperature of $\mathrm{Al}_{2} \mathrm{Cu}$, thereby promoting and increased formation of eutectic $\mathrm{Al}_{2} \mathrm{Cu}$ in the refined alloys. This was significant because it suggested that eutectic liquid was readily available at late stages of solidification in the refined alloys, which in turn increased their resistance to porosity and hot tearing. 


\section{Chapter 6-Conclusions}

This study systematically investigated the conditions required for hot tearing in three distinct B206 casting alloys: unrefined, $0.02 \mathrm{wt} \% \mathrm{Ti}$ and $0.05 \mathrm{wt} \% \mathrm{Ti}$. A direct correlation between alloy solidification, microstructure, grain size, residual strain, phase formation and growth and liquid metal feeding was developed. This novel analysis of hot tearing mechanisms reinforces the developed theory with quantitative data.

One of the eventual objectives of this research is weight reduction in automobiles. Use of Al alloys as alternatives to cast iron entails improved castability. This thesis is a significant contribution towards that goal.

The following major conclusions can be drawn from this research:

\section{Ex Situ Analysis}

The results of ex situ analysis of hot tearing showed good correlation among alloy solidification, microstructure, residual strain and hot tearing in B206. Thermal analysis suggested that grain refinement through additions of Ti delayed the onset temperature and time of coherency in B206. As a result, the duration of bulk liquid feeding was enhanced for the refined casting conditions. In turn, a more uniform distribution of compressive strain was observed along the refined castings, which was critical in increasing the resistance to hot tearing for the refined alloys.

\section{In Situ Analysis}

In situ neutron diffraction solidification analysis successfully determined the distinct phase evolution of $\alpha$-Al solid solution and intermetallic $\mathrm{Al}_{2} \mathrm{Cu}$ during solidification of the unrefined and refined binary Al-5 wt $\% \mathrm{Cu}$ alloys. This was a unique contribution to this research and has not been reported in literature so far. Further, these results provided an improved understanding of the impact of microstructure development on hot tearing in B206. In the case of the unrefined alloy, intermetallic $\mathrm{Al}_{2} \mathrm{Cu}$ precipitated at the instant when the precipitation of $\alpha$ - $\mathrm{Al}$ solid solution was almost complete. As a result, a decreased amount of eutectic liquid was present in the

unrefined alloy, which suggests that late stage feeding was limited during solidification of the 
unrefined alloy. In contrast, the intermetallic $\mathrm{Al}_{2} \mathrm{Cu}$ precipitated at lower solid fractions of $\alpha$-Al in the refined alloys (relative to the unrefined alloy), which suggested that an increased amount of eutectic liquid was readily available to feed the refined castings at late stages of solidification. In turn, the resistance to hot tearing was significantly improved for the refined alloys.

Additional conclusions from this research were as follows:

1. Titanium additions were successful at reducing grain size and transforming grain morphology from coarse dendrites to fine globular grains. This transformation in grain morphology promoted a more uniform distribution of $\mathrm{Al}_{2} \mathrm{Cu}$ intermetallic across the microstructure. This suggested that feeding of liquid metal was more homogeneous in the refined alloys and in turn, increased the resistance to hot tearing for the grain refined B206 castings.

2. Thermal analysis identified the hot spot region along the horizontal bar of the B206 castings, which provided an understanding of the casting solidification profile.

3. Tensile residual $x$-direction $\left(\varepsilon_{x}\right)$ strain was recorded at the junction between the downsprue and the horizontal bar for each casting condition. This tension was sufficient to form hot tears in the unrefined and $0.02 \mathrm{wt} \%$ Ti castings, but did not initiate tears in the $0.05 \mathrm{wt} \%$ Ti casting. Instead, SEM microscopy revealed that the globular grains of this casting condition resisted hot tear formation, as only microcracks were visible.

4. Measurements of strain along the remainder of the horizontal bar revealed mainly compressive $\varepsilon_{x}$ at this region for each casting condition. These compressive strains were due to the inability of the end restraint to hinder $x$-direction casting contraction. Strain profiles along the unrefined casting also illustrated localized tensile $\varepsilon_{x}$ at the hot spot region, which ultimately led to hot tearing for this casting condition.

5. Residual strain measurements in the $y$ and $z$-directions (i.e. $\varepsilon_{y}$ and $\varepsilon_{z}$ ) revealed uniform compressive strain for the refined castings. In contrast, a mix of tensile and compressive 
$\varepsilon_{y}$ and $\varepsilon_{z}$ was measured along the unrefined casting. This suggests that $\varepsilon_{y}$ and $\varepsilon_{z}$ did not significantly contribute to hot tearing in the refined castings but did so in the unrefined casting. The results of $\varepsilon_{y}$ and $\varepsilon_{z}$ were also used to calculate residual stress. The profiles of $x$-direction stress showed good agreement to those of $\varepsilon_{x}$ and demonstrated that tensile $x$-direction strain was more critical than stress at initiating hot tears in B206.

6. Higher mold temperatures were successful at reducing the severity of hot tears in unrefined B206, but did not completely eliminate them. Higher mold temperatures likely enhanced the time available for liquid metal feeding, which in turn, helped to limit the severity of hot tearing.

7. Force-temperature-time curves were generated from the CRC mold equipped with a load cell. The force curves showed good agreement with the cracking severity of B206. The onset temperatures of hot tearing in B206 were determined at $619{ }^{\circ} \mathrm{C}, 602{ }^{\circ} \mathrm{C}$ and $637{ }^{\circ} \mathrm{C}$ for the $250{ }^{\circ} \mathrm{C}, 325^{\circ} \mathrm{C}$ and $400{ }^{\circ} \mathrm{C}$ mold temperatures, respectively. These corresponded to an estimated solid fraction of $0.85,0.87$ and 0.81 . A secondary crack was detected at $610{ }^{\circ} \mathrm{C}$ for the $400{ }^{\circ} \mathrm{C}$ mold temperature corresponding to a solid fraction of 0.85 . The range of onset solid fraction of hot tearing determined in B206 was in agreement with literature.

8. The development of solid $\alpha-\mathrm{Al}$ in the unrefined and grain-refined alloys showed good agreement with their respective grain morphologies. Grain refinement was not seen to impact the growth profile of $\mathrm{Al}_{2} \mathrm{Cu}$ but slightly increased the nucleation temperature of $\mathrm{Al}_{2} \mathrm{Cu}$. 


\section{Chapter 7 - Recommendations for Future Work}

The results of this dissertation help advance the understanding of the underlying mechanisms of hot tearing in B206 aluminum alloy and provide the foundations necessary for continuation of research into the hot tearing of aluminum alloys. Future experiments and analysis are recommended to further enhance the current knowledge of hot tearing with quantitative data. Some recommendations include:

1. Ex situ neutron diffraction strain mapping of the unrestrained B206 casting (i.e. casting with insert placed in casting cavity) to quantify the effect of the end restraint in the permanent mold on residual strain along the casting.

2. Investigations on the effect of casting geometry (e.g. various radii at sprue-bar junction) on residual strain and subsequent hot tearing susceptibility.

3. Implementation of real-time X-ray tomography to observe the evolution of hot tears during alloy solidification. Such images, combined with thermal analysis can give an accurate indication of microstructure at the onset temperature of hot tearing.

4. In situ neutron diffraction solidification analysis on binary Al-5 wt\% $\mathrm{Cu}$ samples using various cooling rates in order to investigate the effect of cooling rate on $\mathrm{Al}_{2} \mathrm{Cu}$ nucleation temperature and growth profile.

5. Rietveld analysis of $\mathrm{Al}_{2} \mathrm{Cu}$ peak evolution to quantify the volume fraction of $\mathrm{Al}_{2} \mathrm{Cu}$ at various temperatures.

6. In situ neutron diffraction solidification analysis of $\mathrm{Al}-5 \mathrm{wt} \% \mathrm{Cu}$ at temperatures beyond $420{ }^{\circ} \mathrm{C}$ to determine a calibration factor accounting for the Debye Waller effect.

7. In situ neutron diffraction strain measurements to determine strain at the onset of hot tearing. Specifically, using the onset temperatures and solid fractions obtained in this research for B206, the strain analysis can be carried out at these temperatures to quantify the strain at the onset of hot tearing for B206. 
Appendix 1 - B206 Composition Analysis 
Table A.1-1. Composition of B206 used for ex situ casting experiments, in wt\%.

\begin{tabular}{|c|c|c|c|c|c|c|c|c|c|c|}
\hline $\begin{array}{c}\text { Target } \\
\text { Ti } \\
\text { Level }\end{array}$ & $\mathbf{C u}$ & $\mathbf{M n}$ & $\mathbf{M g}$ & $\mathbf{F e}$ & $\mathbf{S i}$ & $\mathbf{N i}$ & $\mathbf{Z n}$ & $\mathbf{S n}$ & $\mathbf{T i}$ & $\mathbf{A l}$ \\
\hline Unref. & 4.8 & 0.38 & 0.23 & 0.09 & 0.04 & $<0.01$ & $<0.01$ & $<0.01$ & 0.005 & Bal. \\
\hline $\mathbf{0 . 0 2}$ & 4.7 & 0.39 & 0.22 & 0.13 & 0.02 & $<0.01$ & $<0.01$ & $<0.01$ & 0.02 & Bal. \\
\hline $\mathbf{0 . 0 5}$ & 4.7 & 0.38 & 0.22 & 0.12 & 0.02 & $<0.01$ & $<0.01$ & $<0.01$ & 0.05 & Bal. \\
\hline
\end{tabular}

Table A.1-2. Composition of B206 used for in situ casting experiments, in wt \%.

\begin{tabular}{|c|c|c|c|c|c|c|c|c|c|c|}
\hline $\begin{array}{c}\text { Target } \\
\text { Ti } \\
\text { Level }\end{array}$ & $\mathbf{C u}$ & $\mathbf{M n}$ & $\mathbf{M g}$ & $\mathbf{F e}$ & $\mathbf{S i}$ & $\mathbf{N i}$ & $\mathbf{Z n}$ & $\mathbf{S n}$ & $\mathbf{T i}$ & $\mathbf{A l}$ \\
\hline Unref. & 4.5 & 0.40 & 0.24 & 0.06 & 0.07 & $<0.01$ & $<0.01$ & $<0.01$ & 0.005 & Bal. \\
\hline $\mathbf{0 . 0 2}$ & 4.6 & 0.37 & 0.21 & 0.05 & 0.10 & $<0.01$ & $<0.01$ & $<0.01$ & 0.02 & Bal. \\
\hline $\mathbf{0 . 0 5}$ & 4.6 & 0.33 & 0.20 & 0.05 & 0.35 & $<0.01$ & $<0.01$ & $<0.01$ & 0.06 & Bal. \\
\hline
\end{tabular}

Table A.1-3. Composition of binary Al-5 wt $\% \mathrm{Cu}$ alloy used for in situ neutron diffraction solidification analysis, in wt\%.

\begin{tabular}{|c|c|c|c|c|c|c|c|c|c|c|}
\hline $\begin{array}{c}\text { Target } \\
\text { Ti } \\
\text { Level }\end{array}$ & $\mathbf{C u}$ & $\mathbf{M n}$ & $\mathbf{M g}$ & $\mathbf{F e}$ & $\mathbf{S i}$ & $\mathbf{N i}$ & $\mathbf{Z n}$ & $\mathbf{S n}$ & $\mathbf{T i}$ & $\mathbf{A l}$ \\
\hline Unref. & 5.2 & $<0.01$ & 0.04 & 0.09 & 0.06 & $<0.01$ & $<0.01$ & $<0.01$ & 0.007 & Bal. \\
\hline $\mathbf{0 . 0 2}$ & 5.2 & $<0.01$ & 0.04 & 0.13 & 0.08 & $<0.01$ & $<0.01$ & $<0.01$ & 0.02 & Bal. \\
\hline $\mathbf{0 . 0 5}$ & 5.2 & $<0.01$ & 0.04 & 0.11 & 0.10 & $<0.01$ & $<0.01$ & $<0.01$ & 0.05 & Bal. \\
\hline
\end{tabular}


Appendix 2-Micrographs at Hot Tear Region 


\section{A2.1 Unrefined Alloy}

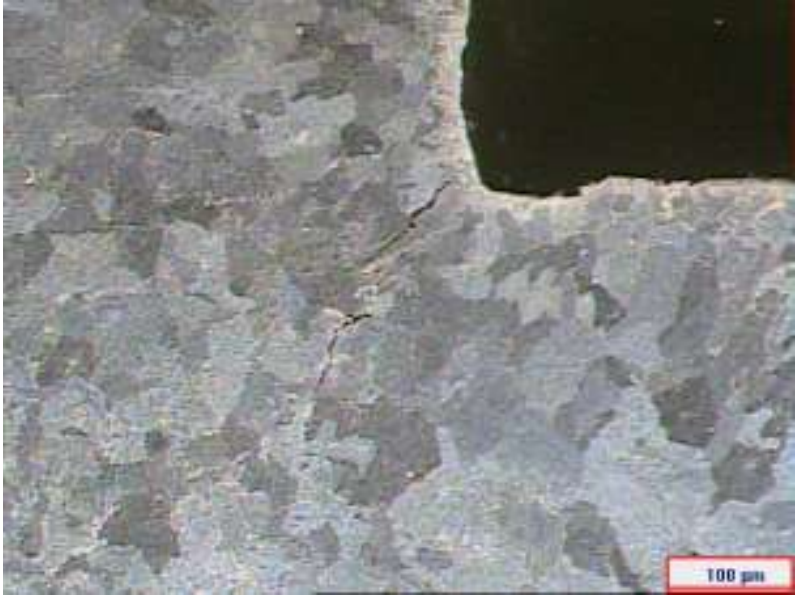

a)

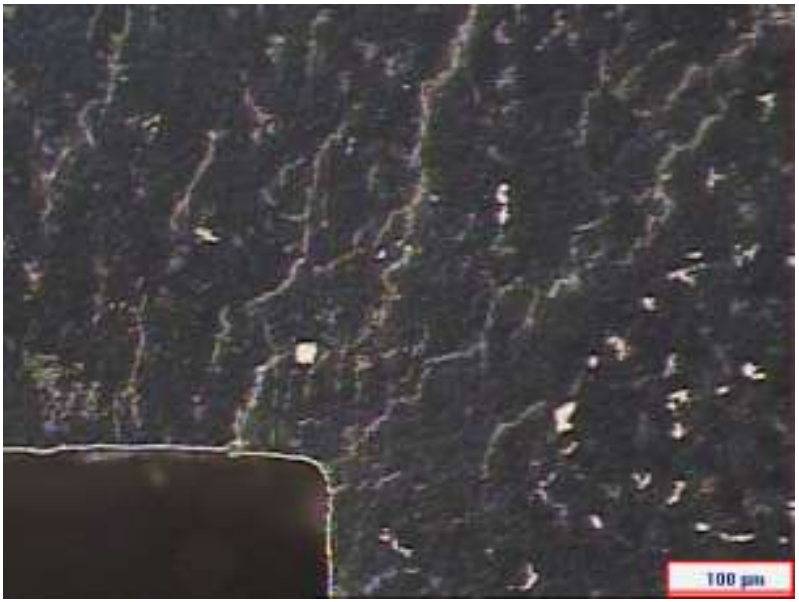

c)

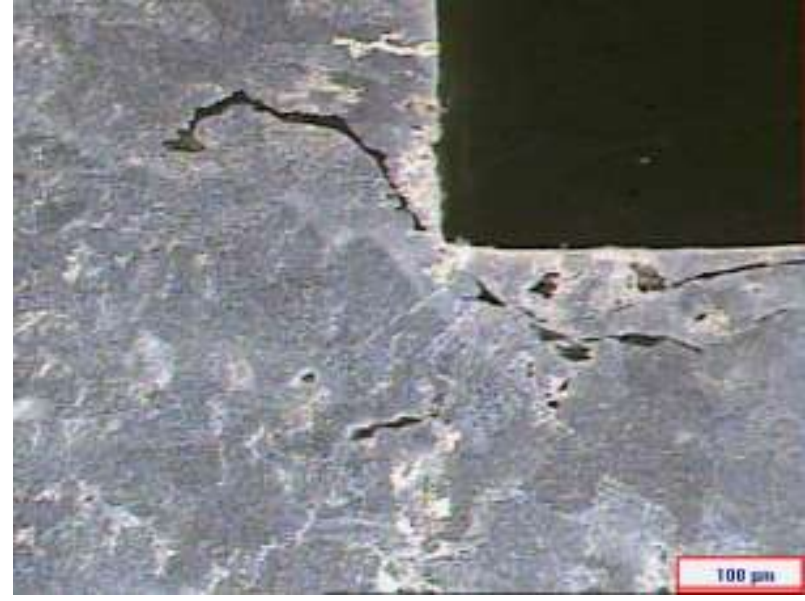

b)

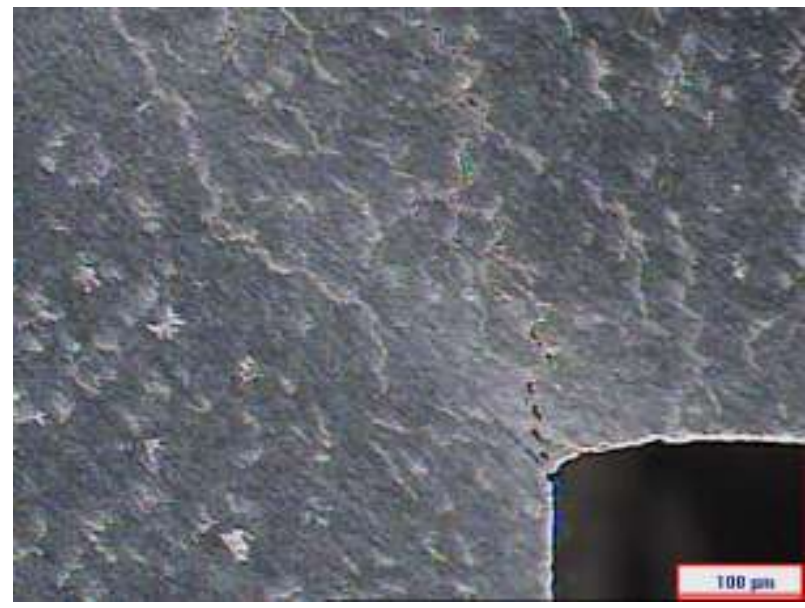

d)

Figure A.2-1. Hot tears along a) Sample \#1; b) Sample \#3; c) Sample \#2 and d) Sample \#4 at the sprue-bar junction of the unrefined B206 alloy. 


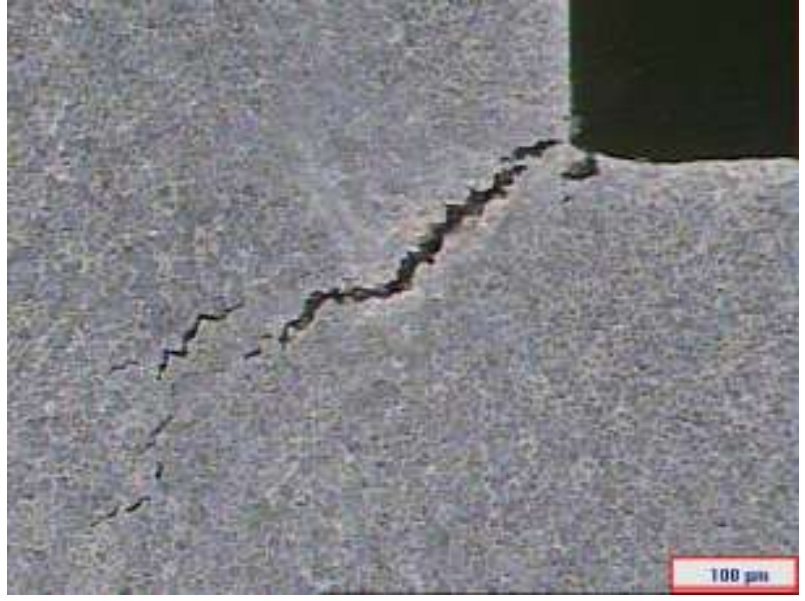

a)

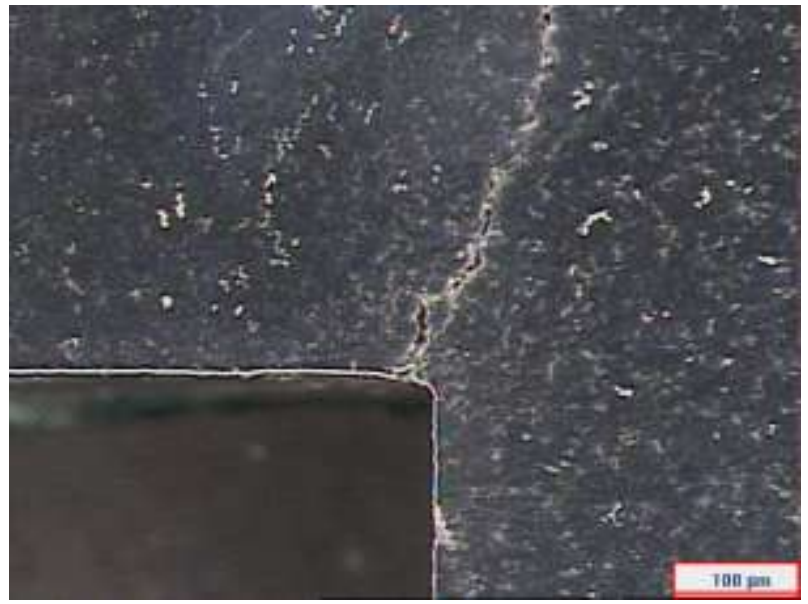

(c)

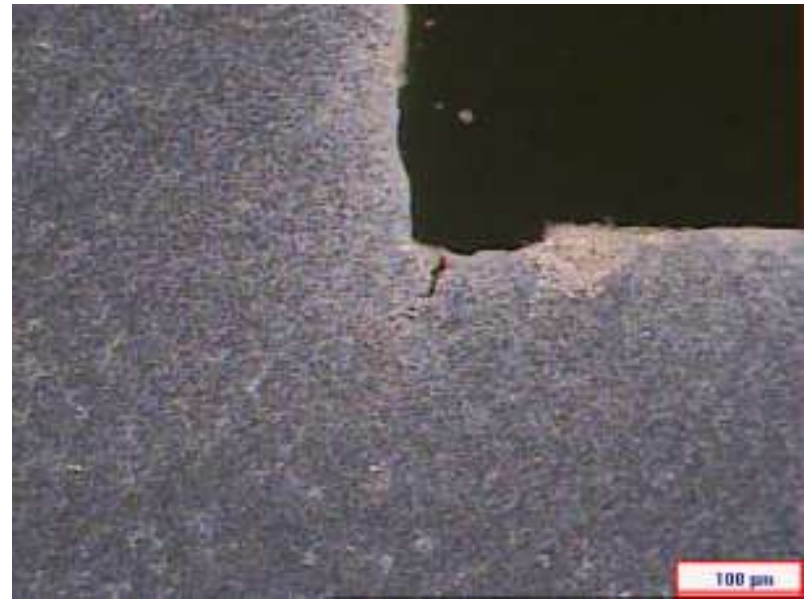

b)

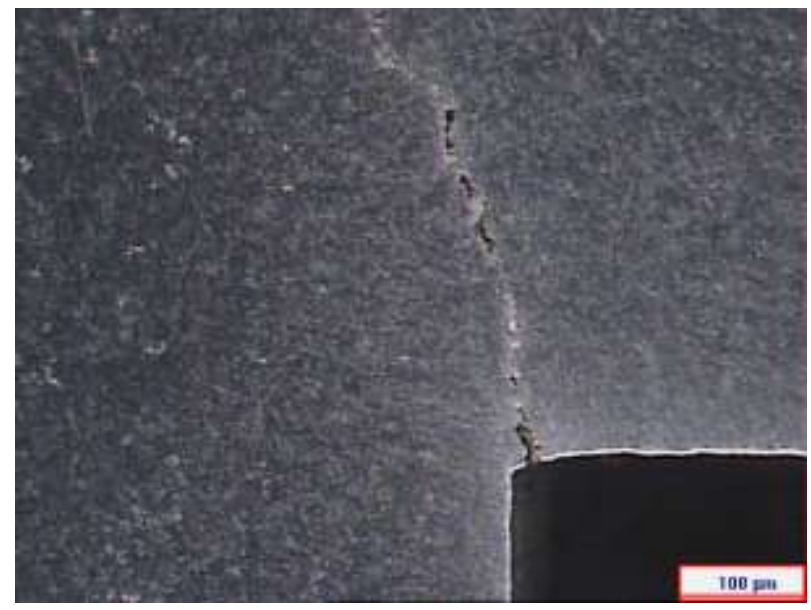

(d)

Figure A.2-2. Hot tears along a) Sample \#1; b) Sample \#3; c) Sample \#2 and d) Sample \#4 at the sprue-bar junction of the $0.02 \mathrm{wt} \% \mathrm{Ti}$ alloy. 


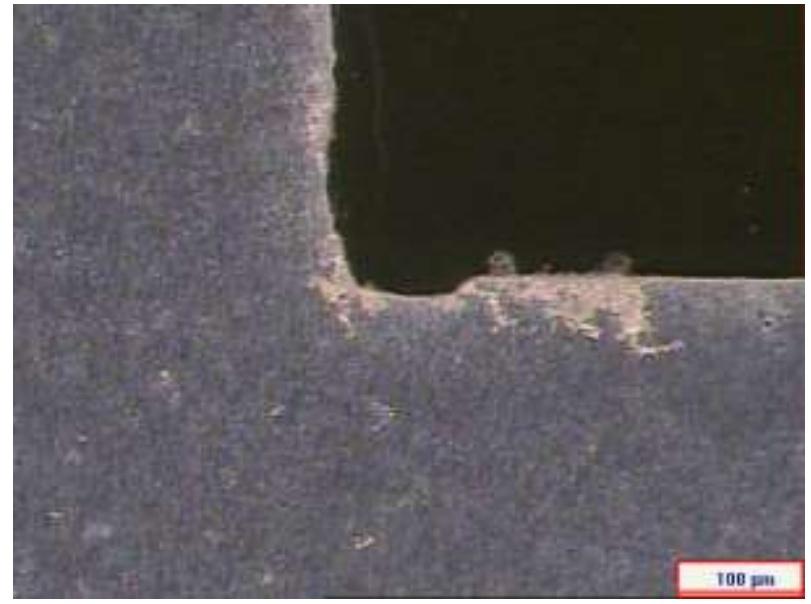

a)

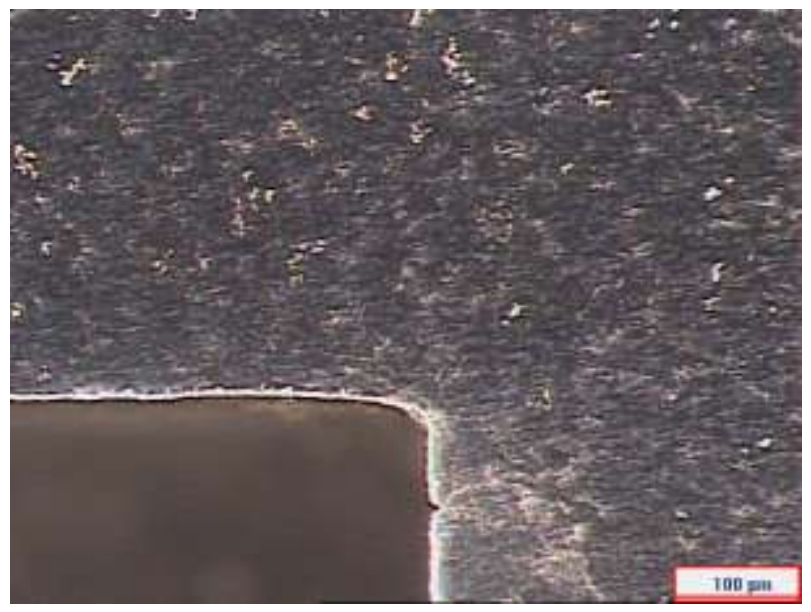

c)

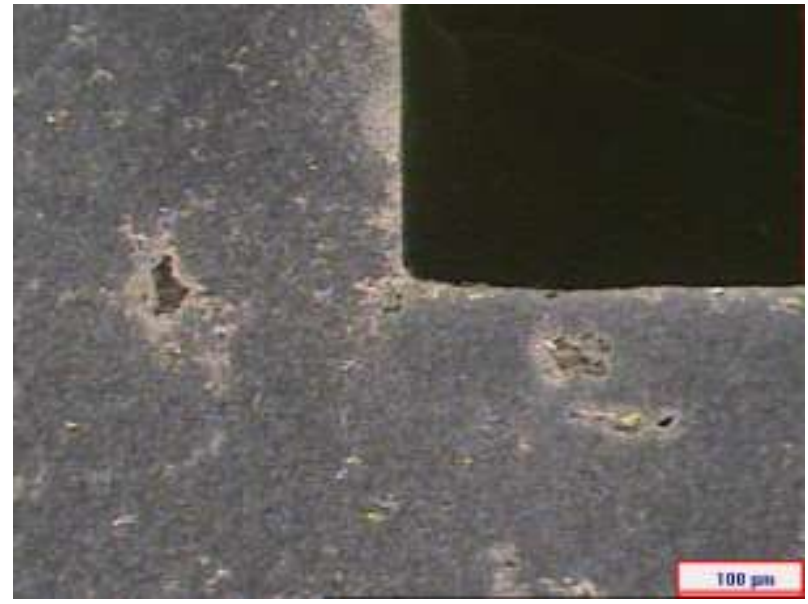

b)

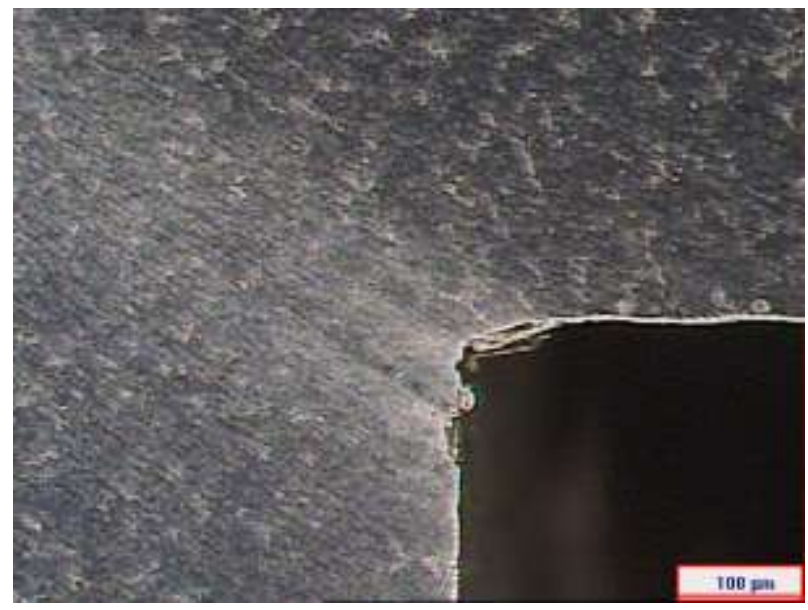

d)

Figure A.2-3. Hot tears along a) Sample \#1; b) Sample \#3; c) Sample \#2 and d) Sample \#4 at the sprue-bar junction of the $0.05 \mathrm{wt} \% \mathrm{Ti}$ alloy. 
Appendix 3-Cooling Curves and Thermal Analysis 


\section{A3.1 Cooling Curves}

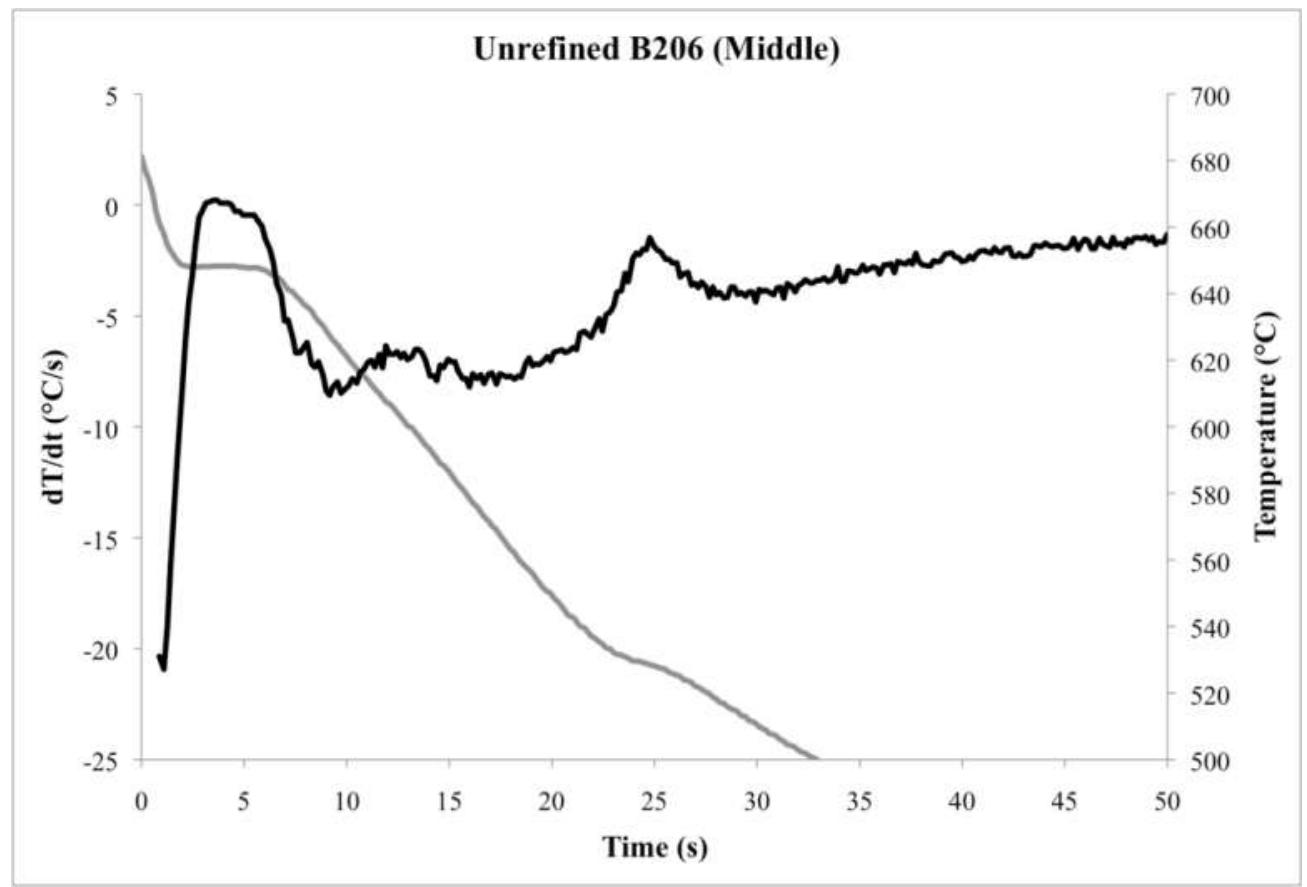

Figure A.3-1. Cooling curve and its first derivative for the unrefined B206 alloy (by the middle of the horizontal bar).

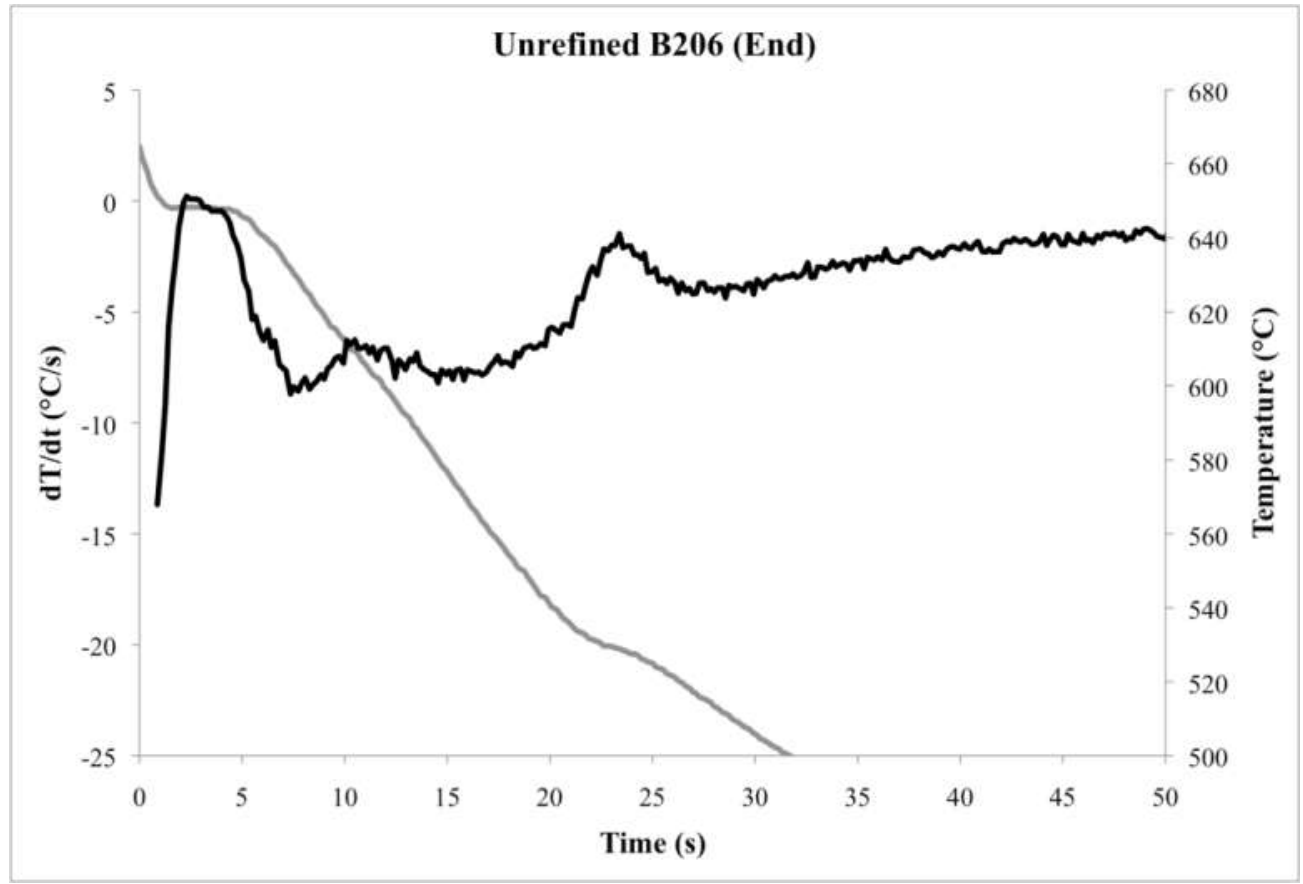

Figure A.3-2. Cooling curve and its first derivative for the unrefined B206 alloy (by the end restraint). 


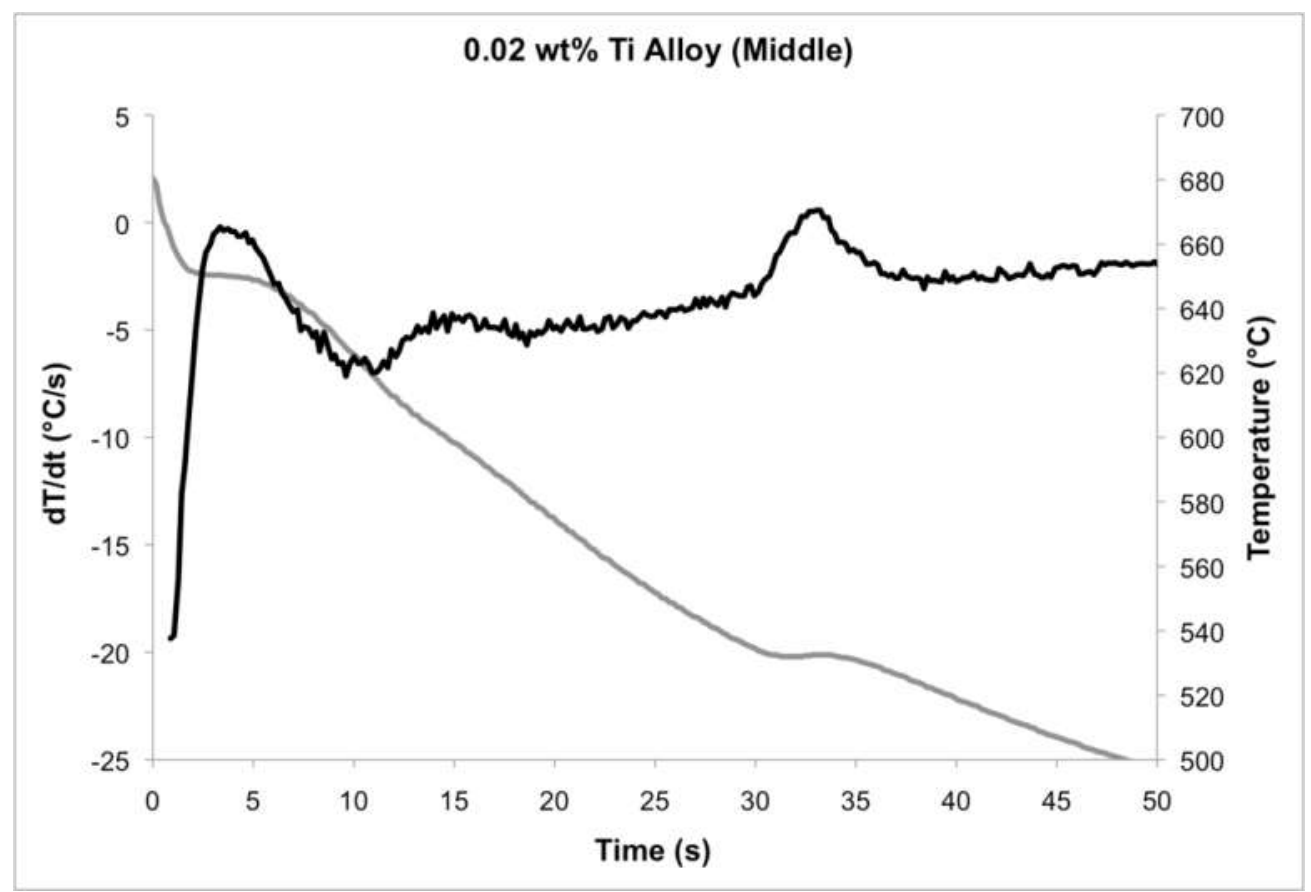

Figure A.3-3. Cooling curve and its first derivative for the 0.02 wt $\%$ Ti alloy (by the middle of the horizontal bar).

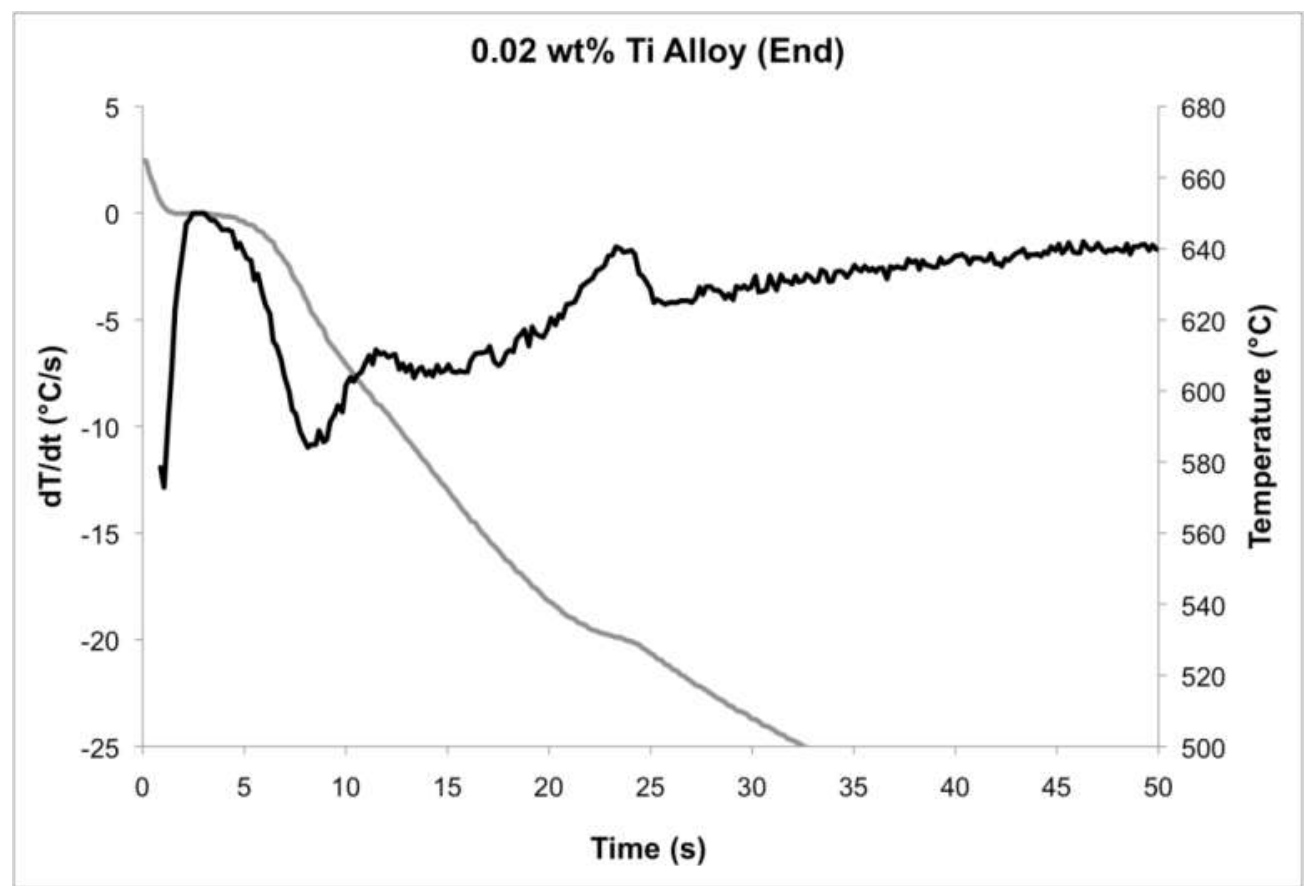

Figure A.3-4. Cooling curve and its first derivative for the $0.02 \mathrm{wt} \% \mathrm{Ti}$ alloy (by the end restraint). 


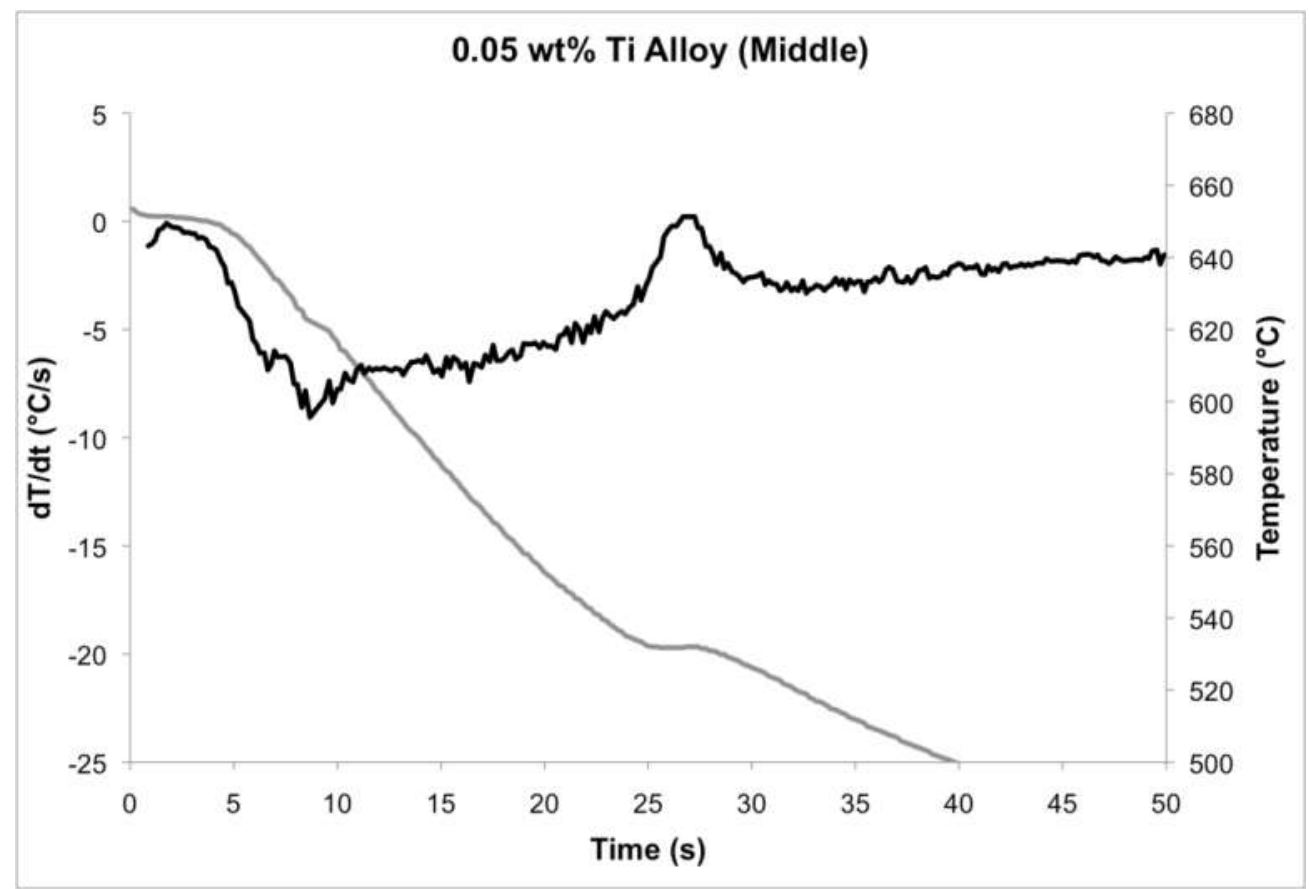

Figure A.3-5. Cooling curve and its first derivative for the $0.05 \mathrm{wt} \%$ Ti alloy (by the middle of the horizontal bar).

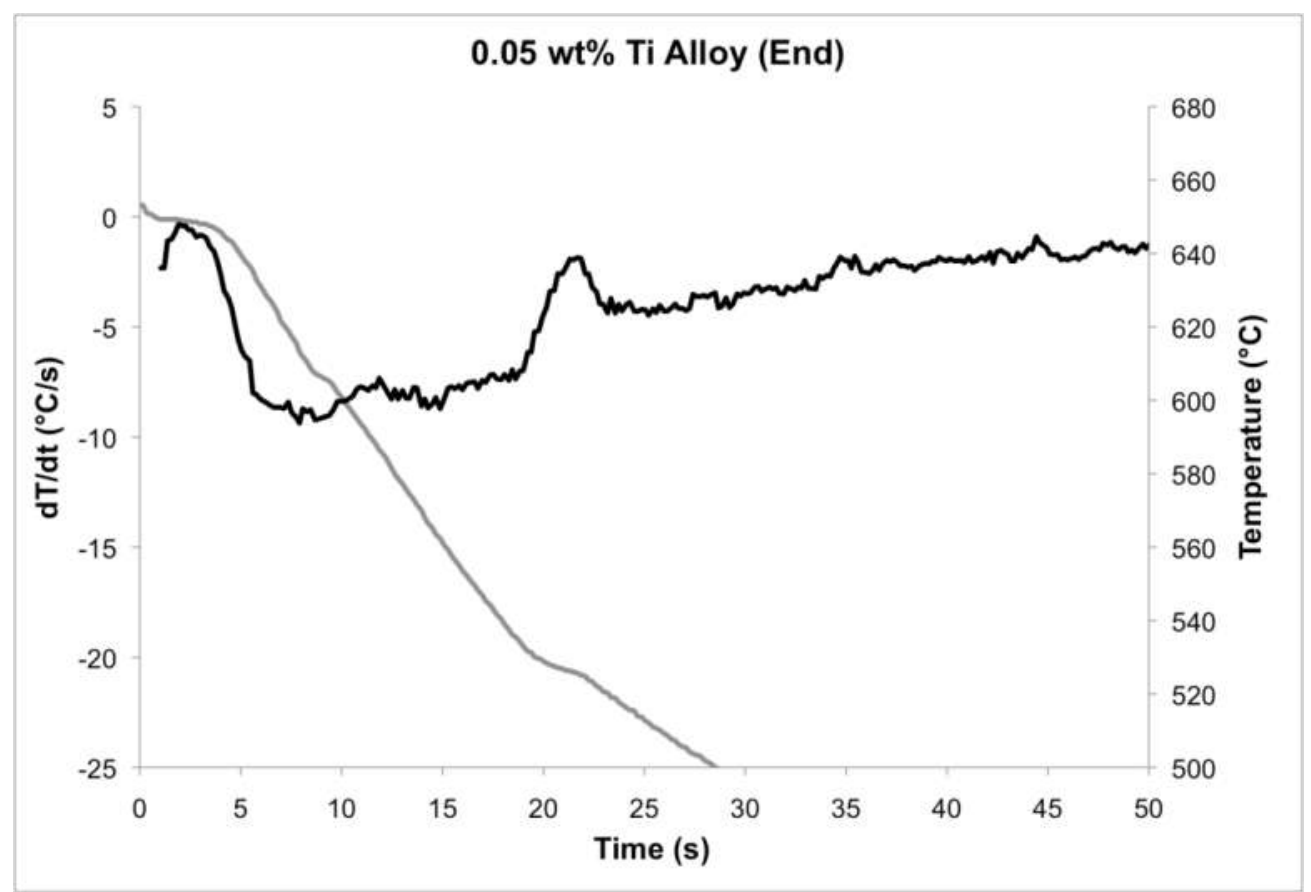

Figure A.3-6. Cooling curve and its first derivative for the $0.05 \mathrm{wt} \% \mathrm{Ti}$ alloy (by the end restraint). 


\section{A3.2 Thermal Analysis}

Table A.3-1. Thermal analysis data (by the middle of the horizontal bar).

\begin{tabular}{|c|c|c|c|c|c|c|c|c|}
\hline \multirow{2}{*}{ Condition } & \multicolumn{2}{|c|}{ Nucleation } & \multicolumn{2}{c|}{ Growth } & \multicolumn{2}{c|}{ Coherency } & \multicolumn{2}{c|}{ Solidification } \\
\cline { 2 - 9 } & $\begin{array}{c}\boldsymbol{T}_{\boldsymbol{N}} \\
\left({ }^{\circ} \mathbf{C}\right)\end{array}$ & $\begin{array}{c}\boldsymbol{t}_{\boldsymbol{N}} \\
(\mathbf{s})\end{array}$ & $\begin{array}{c}\boldsymbol{T}_{\boldsymbol{G}} \\
\left({ }^{\circ} \mathbf{C}\right)\end{array}$ & $\begin{array}{c}\boldsymbol{t}_{\boldsymbol{G}} \\
(\mathbf{s})\end{array}$ & $\begin{array}{c}\boldsymbol{T}_{\boldsymbol{C}} \\
\left({ }^{\circ} \mathbf{C}\right)\end{array}$ & $\begin{array}{c}\boldsymbol{t}_{C} \\
(\mathbf{s})\end{array}$ & $\begin{array}{c}\boldsymbol{T}_{\boldsymbol{S}} \\
\left({ }^{\circ} \mathbf{C}\right)\end{array}$ & $\begin{array}{c}\boldsymbol{t}_{\boldsymbol{S}} \\
(\mathbf{s})\end{array}$ \\
\hline Unrefined & 648.0 & 2.5 & 648.5 & 5.0 & 638.0 & 8.0 & 533.0 & 24.5 \\
\hline $0.02 \mathrm{wt} \% \mathrm{Ti}$ & 653.0 & 1.5 & 650.5 & 3.5 & 626.5 & 10.0 & 534.0 & 27.0 \\
\hline $0.05 \mathrm{wt} \% \mathrm{Ti}$ & 653.5 & 0.5 & 651.0 & 3.0 & 622.5 & 10.0 & 533.5 & 25.0 \\
\hline
\end{tabular}

Table A.3-2. Differences in time at points of interest.

\begin{tabular}{|c|c|c|}
\hline Condition & $\boldsymbol{t}_{\boldsymbol{C}}-\boldsymbol{t}_{\boldsymbol{N}}(\mathbf{s})$ & $\boldsymbol{t}_{\boldsymbol{S}}-\boldsymbol{t}_{\boldsymbol{N}}(\mathbf{s})$ \\
\hline Unrefined & 5.5 & 22.0 \\
\hline $0.02 \mathrm{wt} \% \mathrm{Ti}$ & 8.5 & 25.5 \\
\hline $0.05 \mathrm{wt} \% \mathrm{Ti}$ & 9.5 & 24.5 \\
\hline
\end{tabular}

Table A.3-3. Thermal analysis data (by the end restraint).

\begin{tabular}{|c|c|c|c|c|c|c|c|c|}
\hline \multirow{2}{*}{ Condition } & \multicolumn{2}{|c|}{ Nucleation } & \multicolumn{2}{c|}{ Growth } & \multicolumn{2}{c|}{ Coherency } & \multicolumn{2}{c|}{ Solidification } \\
\cline { 2 - 9 } & $\begin{array}{c}\boldsymbol{T}_{\boldsymbol{N}} \\
\left({ }^{\circ} \mathbf{C}\right)\end{array}$ & $\begin{array}{c}\boldsymbol{t}_{N} \\
(\mathbf{s})\end{array}$ & $\begin{array}{c}\boldsymbol{T}_{\boldsymbol{G}} \\
\left({ }^{\circ} \mathbf{C}\right)\end{array}$ & $\begin{array}{c}\boldsymbol{t}_{G} \\
(\mathbf{s})\end{array}$ & $\begin{array}{c}\boldsymbol{T}_{\boldsymbol{C}} \\
\left({ }^{\circ} \mathbf{C}\right)\end{array}$ & $\begin{array}{c}\boldsymbol{t}_{C} \\
(\mathbf{s})\end{array}$ & $\begin{array}{c}\boldsymbol{T}_{S} \\
\left({ }^{\circ} \mathbf{C}\right)\end{array}$ & $\begin{array}{c}\boldsymbol{t}_{S} \\
(\mathbf{s})\end{array}$ \\
\hline Unrefined & 645.5 & 2.0 & 645.5 & 4.0 & 636.0 & 6.0 & 531.0 & 21.5 \\
\hline $0.02 \mathrm{wt} \% \mathrm{Ti}$ & 653.0 & 1.0 & 650.0 & 3.0 & 625.0 & 8.5 & 534.0 & 22.0 \\
\hline $0.05 \mathrm{wt} \% \mathrm{Ti}$ & 653.0 & 0.5 & 649.0 & 2.5 & 624.0 & 8.0 & 533.0 & 19.5 \\
\hline
\end{tabular}

Table A.3-4. Differences in time at points of interest.

\begin{tabular}{|c|c|c|}
\hline Condition & $\boldsymbol{t}_{\boldsymbol{C}}-\boldsymbol{t}_{\boldsymbol{N}}(\mathbf{s})$ & $\boldsymbol{t}_{\boldsymbol{S}}-\boldsymbol{t}_{\boldsymbol{N}}(\mathbf{s})$ \\
\hline Unrefined & 4.0 & 19.5 \\
\hline $0.02 \mathrm{wt} \% \mathrm{Ti}$ & 7.5 & 21.0 \\
\hline $0.05 \mathrm{wt} \% \mathrm{Ti}$ & 7.5 & 19.0 \\
\hline
\end{tabular}


Appendix 4-Residual Strain Profiles 


\section{A4.1 Residual Strain at the Sprue-Bar Junction}

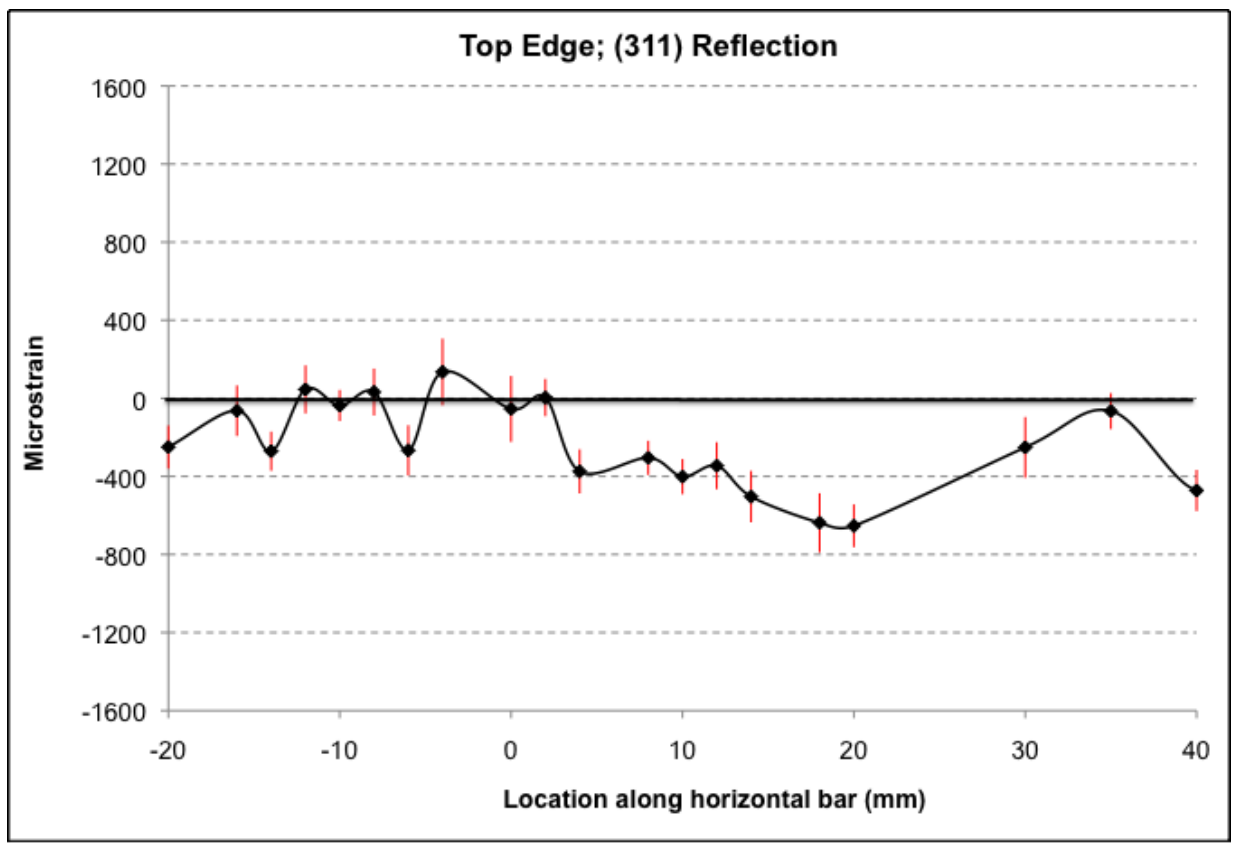

a)

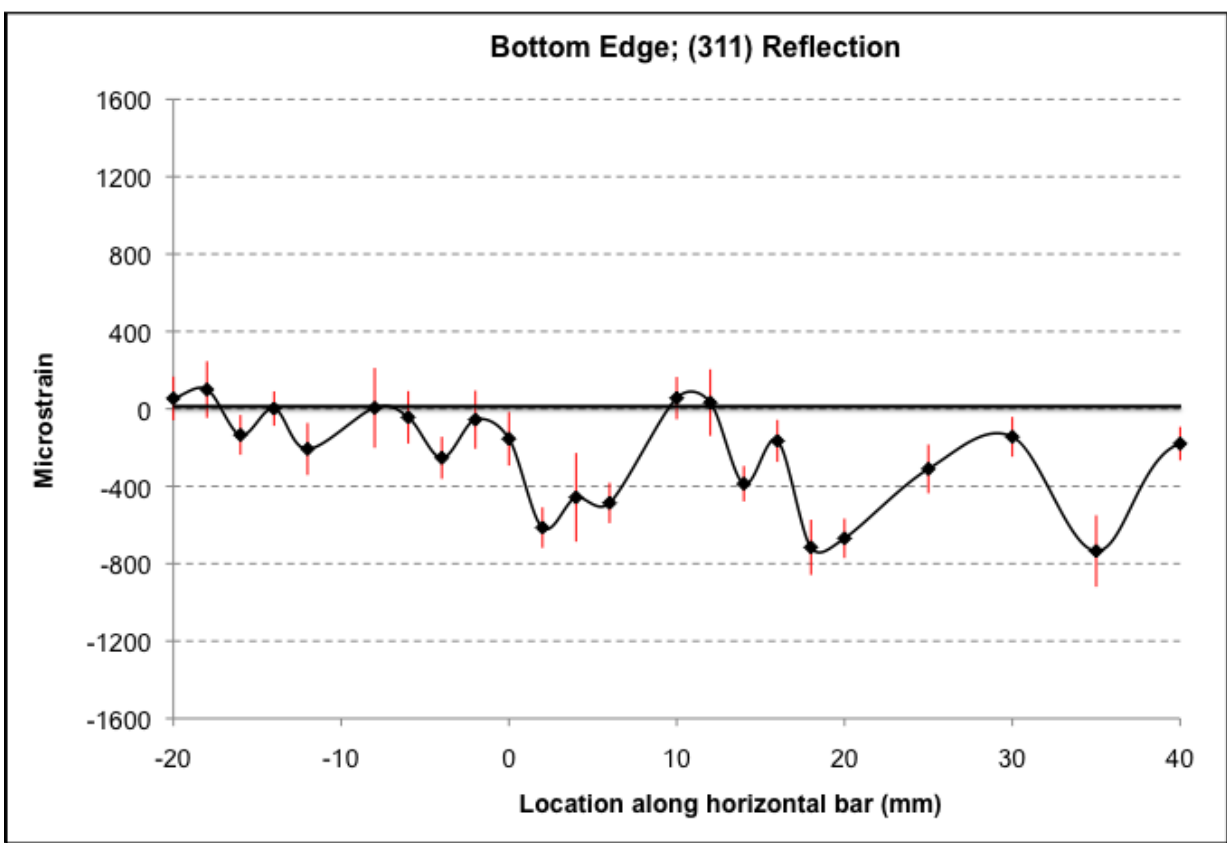

b)

Figure A.4-1. Profiles of $\varepsilon_{x}$ at the sprue-bar junction along the a) top edge and b) bottom edge of the unrefined B206 alloy's horizontal bar for the (311) reflection. 


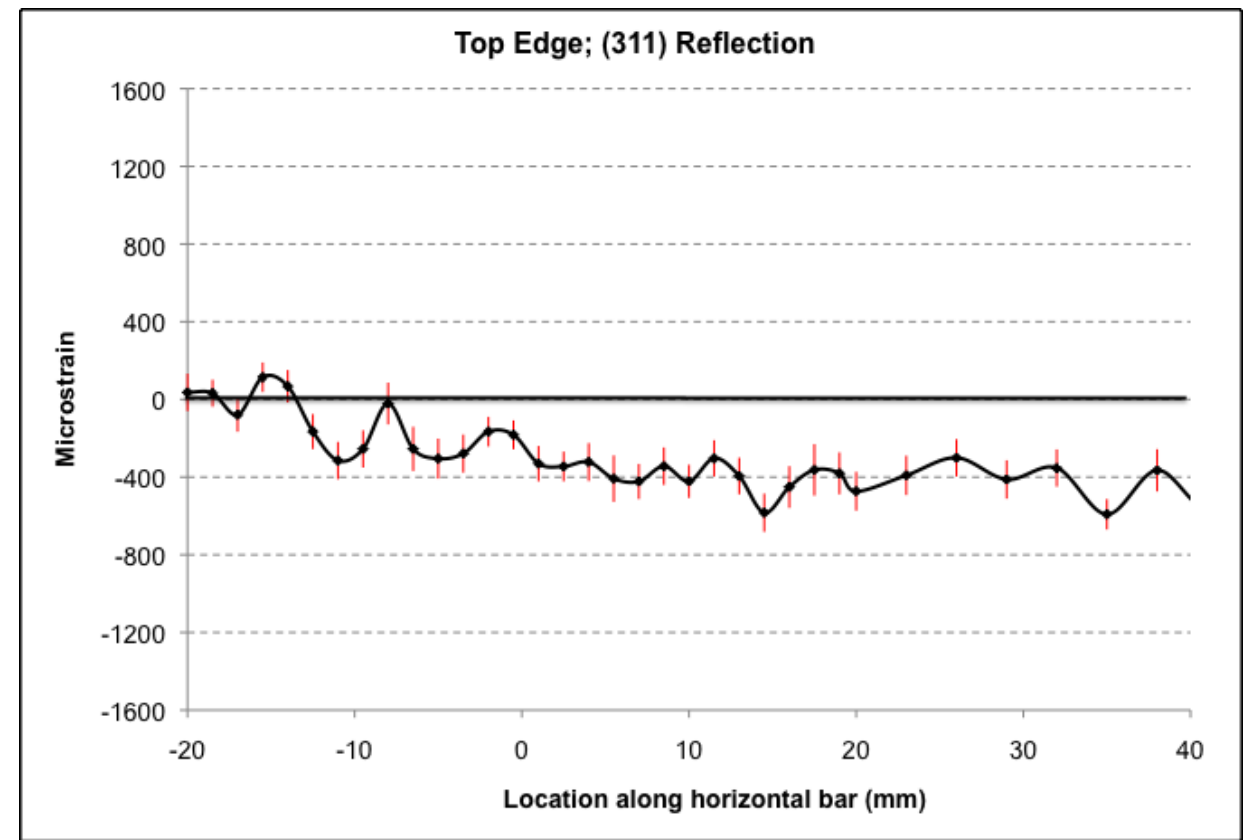

a)

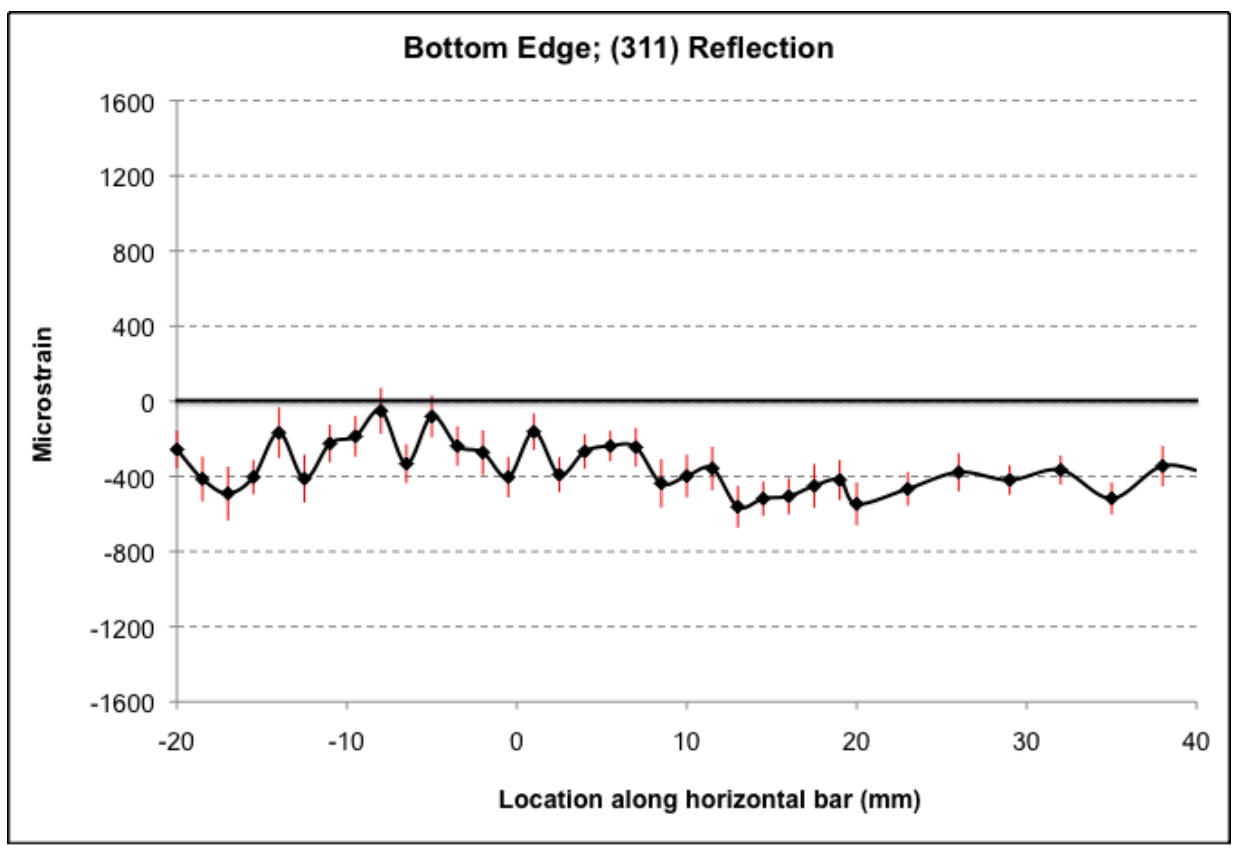

b)

Figure A.4-2. Profiles of $\varepsilon_{x}$ at the sprue-bar junction along the a) top edge and b) bottom edge of the $0.02 \mathrm{wt} \%$ Ti alloy's horizontal bar for the (311) reflection. 


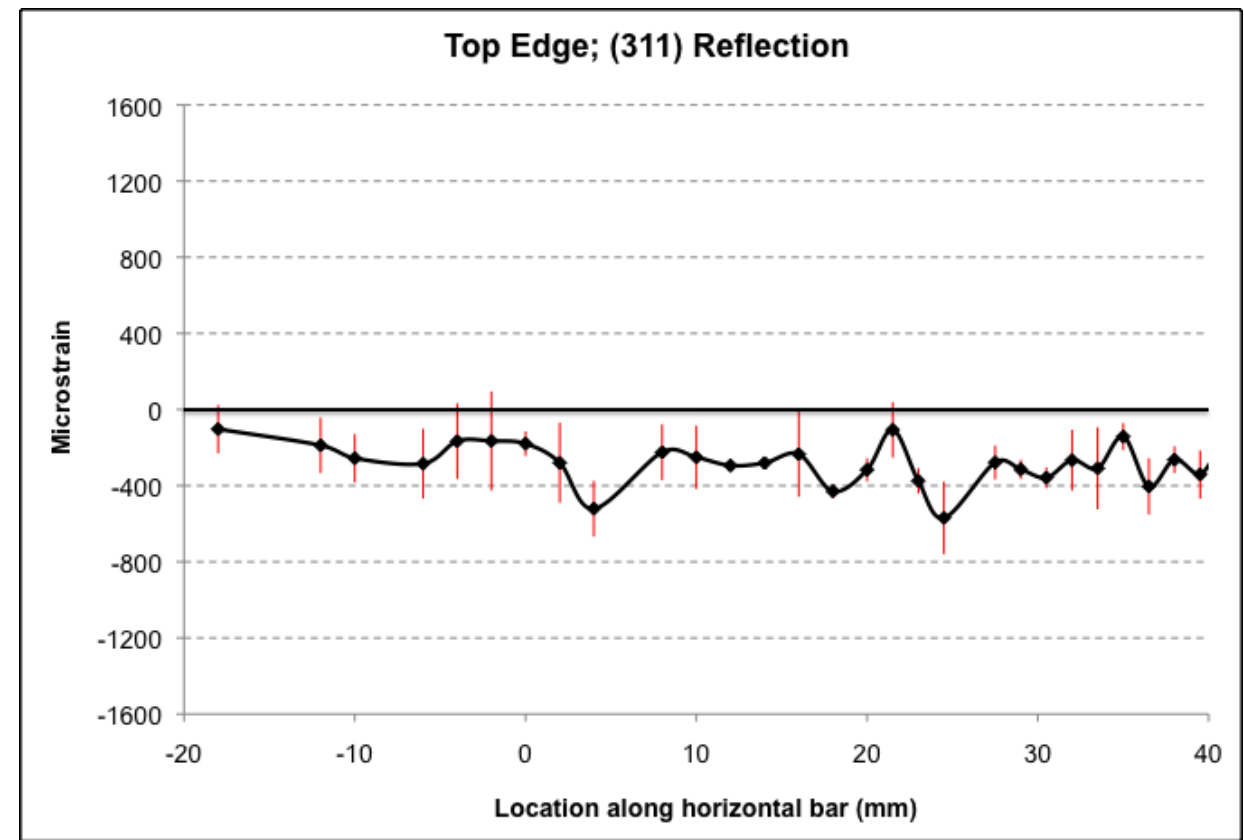

a)

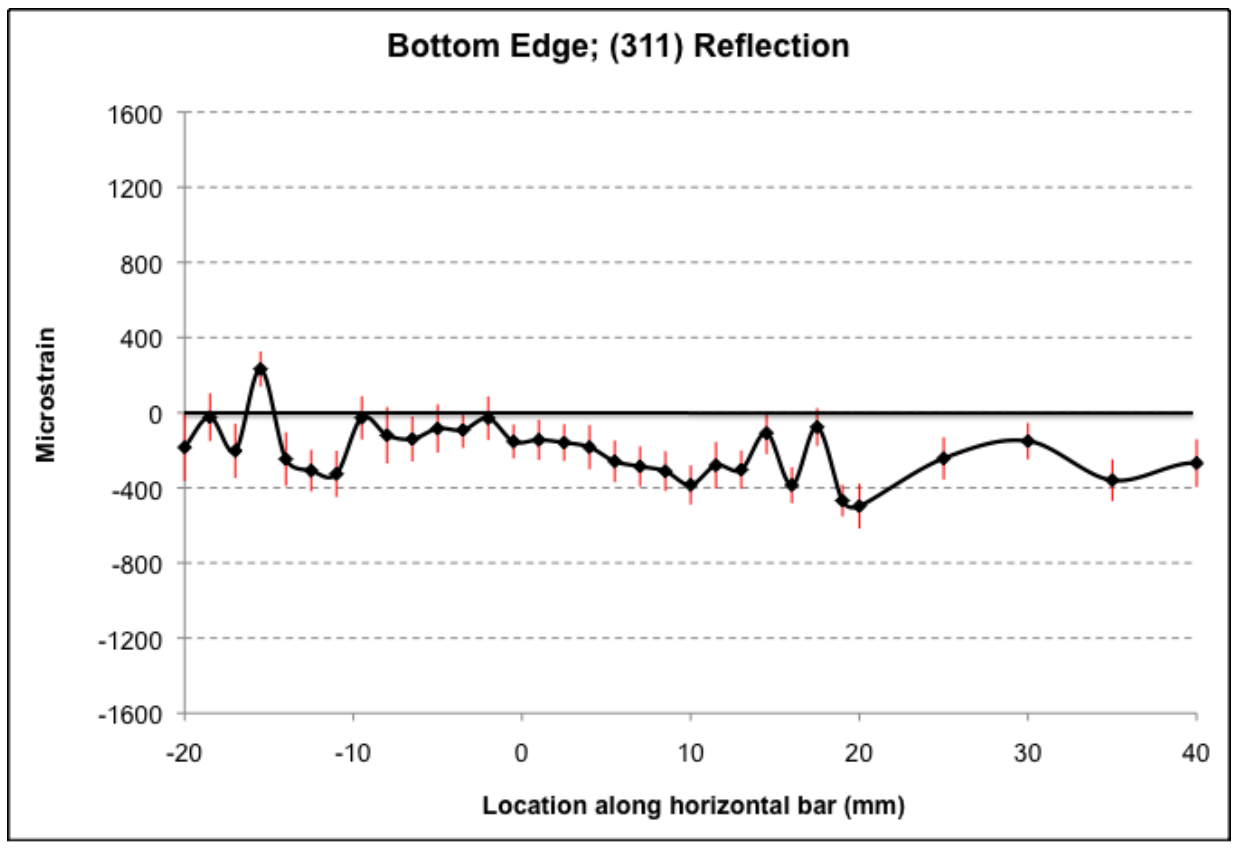

b)

Figure A.4-3. Profiles of $\varepsilon_{x}$ at the sprue-bar junction along the a) top edge and b) bottom edge of the $0.05 \mathrm{wt} \%$ Ti alloy's horizontal bar for the (311) reflection. 


\section{A4.2 Residual Strain Along the Horizontal Bar}

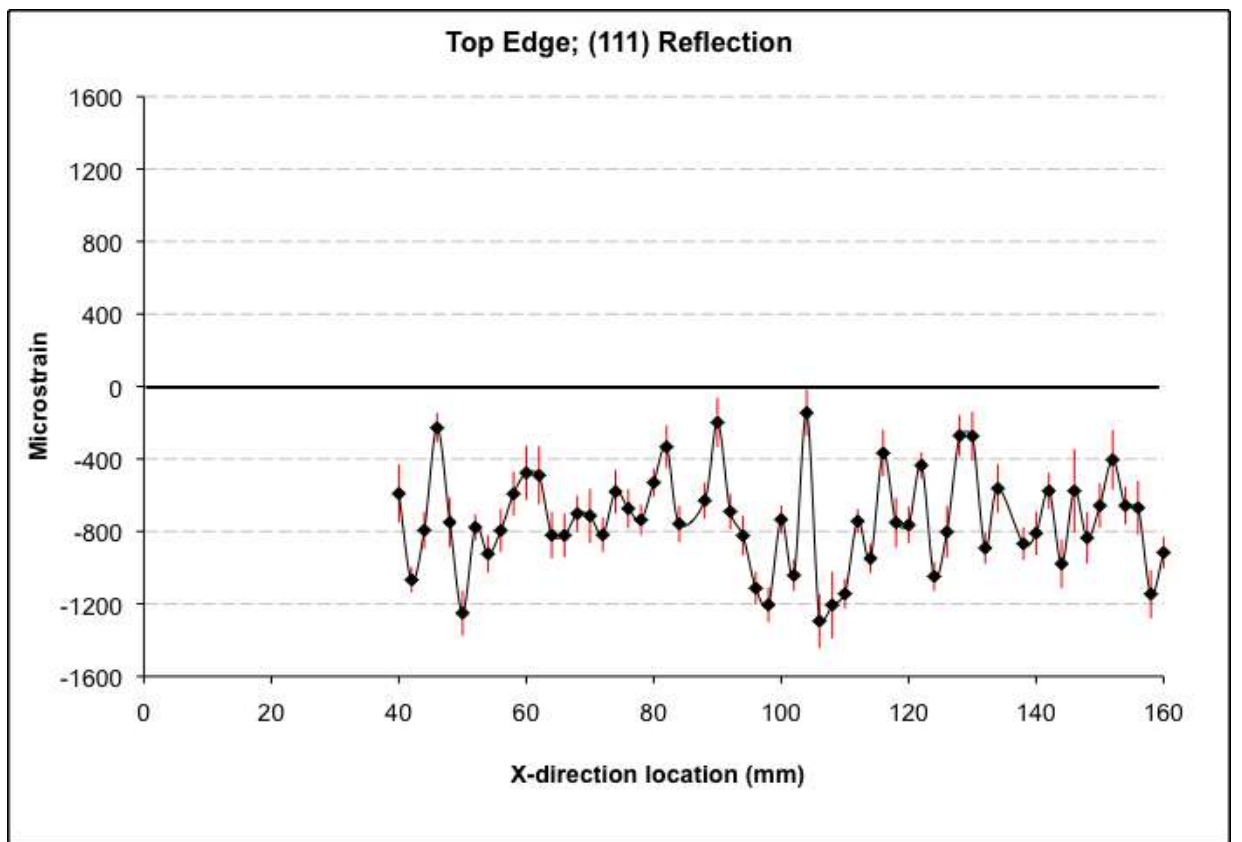

a)

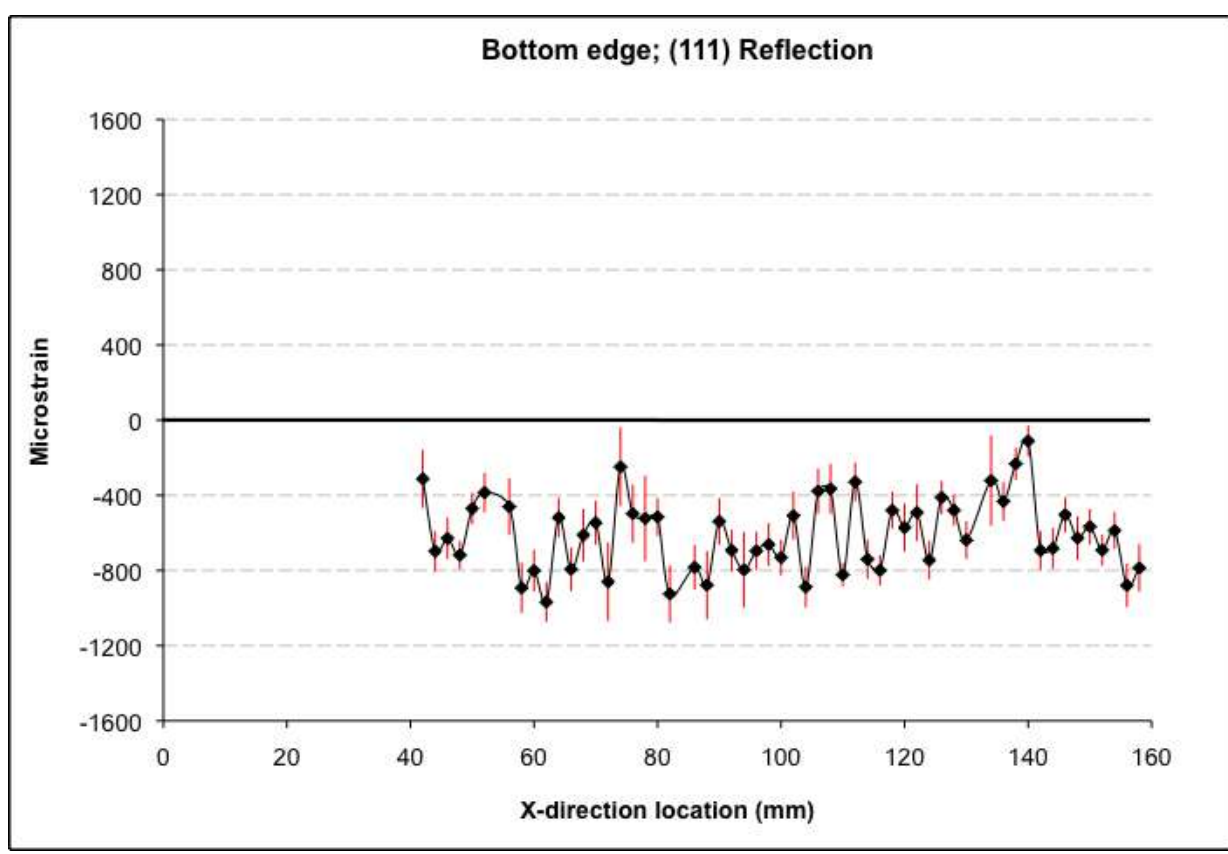

b)

Figure A.4-4. Profiles of $\varepsilon_{x}$ along the a) top edge and b) bottom edge of the horizontal bar for the (111) reflection of the unrefined B206 alloy. 


\section{A4.3 Uncertainty in Residual Strain Calculations}

The diffraction peak (i.e. counts vs. scattering angle) from neutron diffraction strain mapping was fitted to a Gaussian profile by a Fortran program developed at CNBC. Two specific fit parameters, namely the Peak Position and Uncertainty in Peak Position were used to calculate residual strain, $\varepsilon$, and uncertainty in residual strain, $\Delta \varepsilon$. The Peak Position corresponded to the central position of the diffraction peak according to the fit of the data and was therefore taken as the scattering angle, $2 \theta$, while the Uncertainty corresponded to the uncertainty in $2 \theta, \Delta 2 \theta$. The uncertainty in lattice spacing, $\Delta d$, was then calculated using the following equation:

$\Delta d=\frac{n \lambda}{2 \sin (\theta+\Delta \theta)}-d$

Where:

$\theta$ is the Bragg angle ( $1 / 2$ of the scattering angle)

$\Delta \theta$ is the uncertainty in the Bragg angle (1/2 of the uncertainty in scattering angle)

$d=\frac{n \lambda}{2 \sin \theta}$ (the lattice spacing)

From $\Delta d, \Delta \varepsilon$ was calculated as follows:

$\Delta \varepsilon=\frac{(d+\Delta d)-d_{0}}{d_{0}}-\varepsilon$

Where:

$d_{0}$ is the stress-free lattice spacing

$\varepsilon=\frac{d-d_{0}}{d_{0}}$

A similar methodology was used to calculate the uncertainty in residual stress, $\Delta \sigma$, from $\Delta \varepsilon$ and Equation 5. 
Appendix 5-Force-Temperature-Time Curves 


\section{A5.1 Unrefined Alloy}

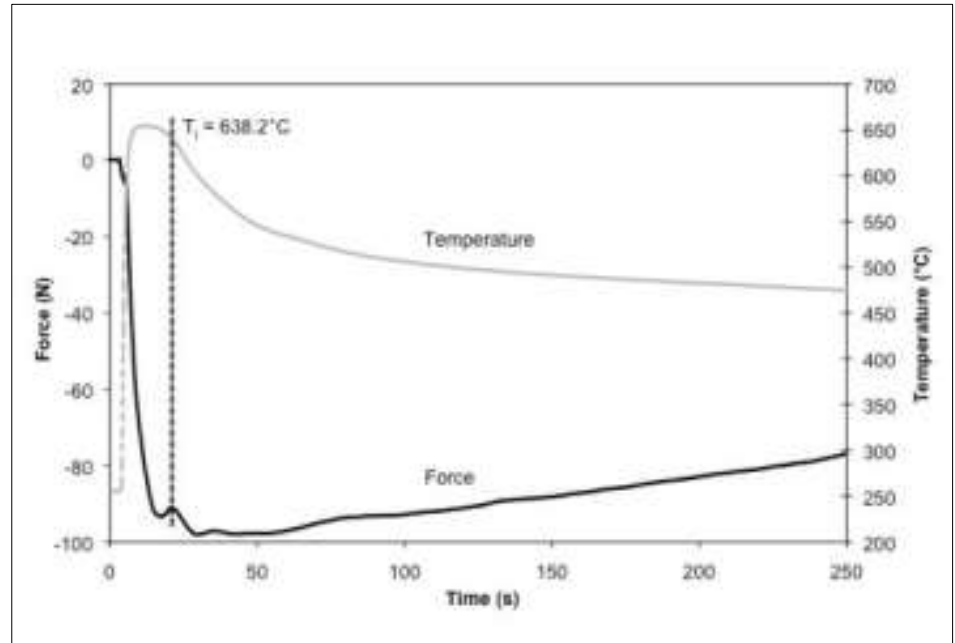

a)

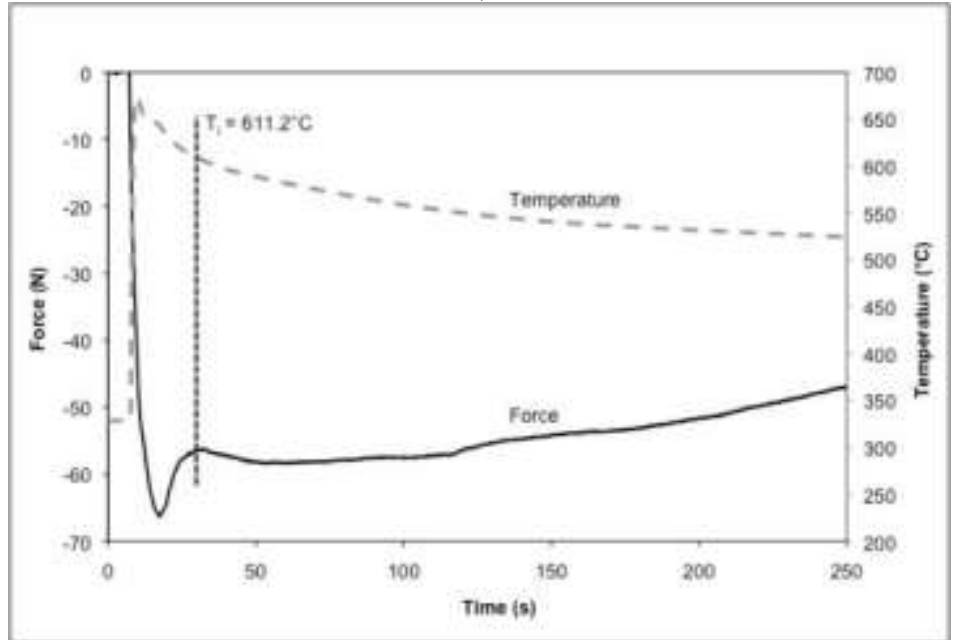

b)

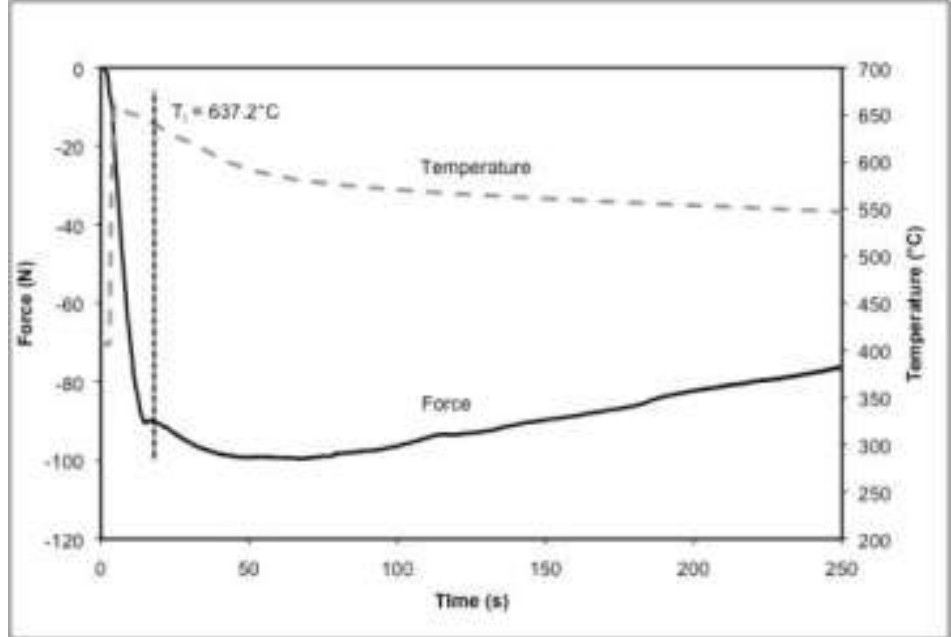

c)

Figure A.5-1. Force-temperature-time curves for unrefined $\mathrm{B206}$ at a) $250{ }^{\circ} \mathrm{C}$; b) $325{ }^{\circ} \mathrm{C}$ and c) $400{ }^{\circ} \mathrm{C}$ mold temperatures. 


\section{A5.2 Refined Alloys}

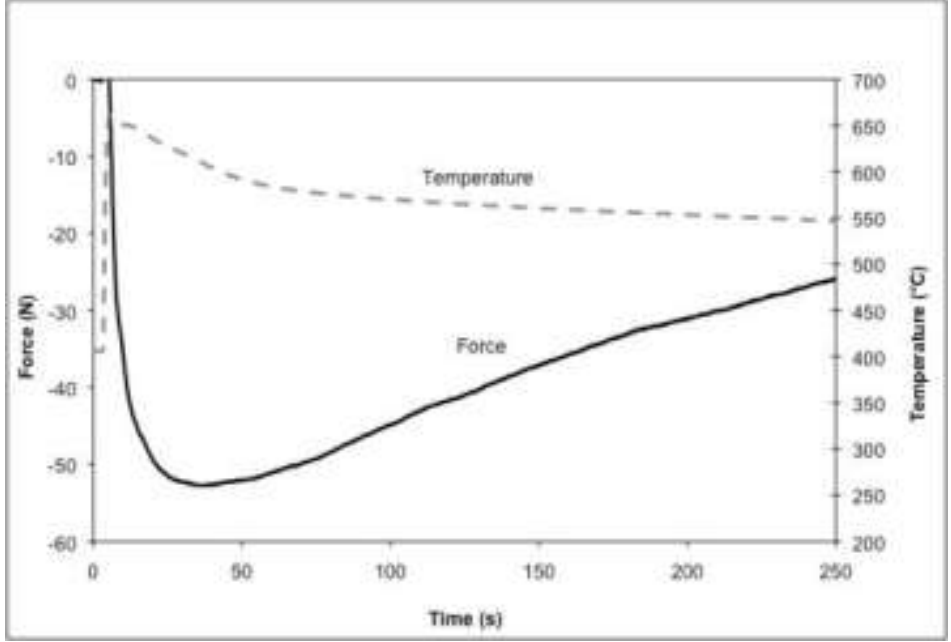

a)

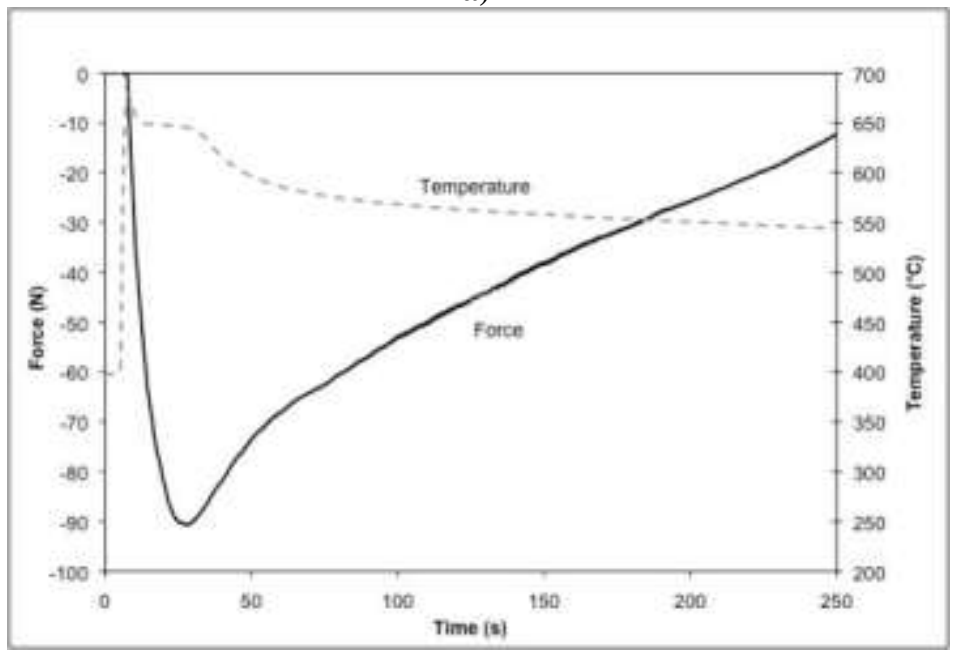

b)

Figure A.5-2. Force-temperature-time curves for a) 0.02 wt \% Ti alloy and b) 0.05 wt $\%$ Ti alloy. 
Appendix 6-Peak Evolution of Primary Aluminum and Intermetallic $\mathrm{Al}_{2} \mathrm{Cu}$ 


\section{A6.1 Primary Al $\mathbf{l}_{(11)}$ Peak Evolution}

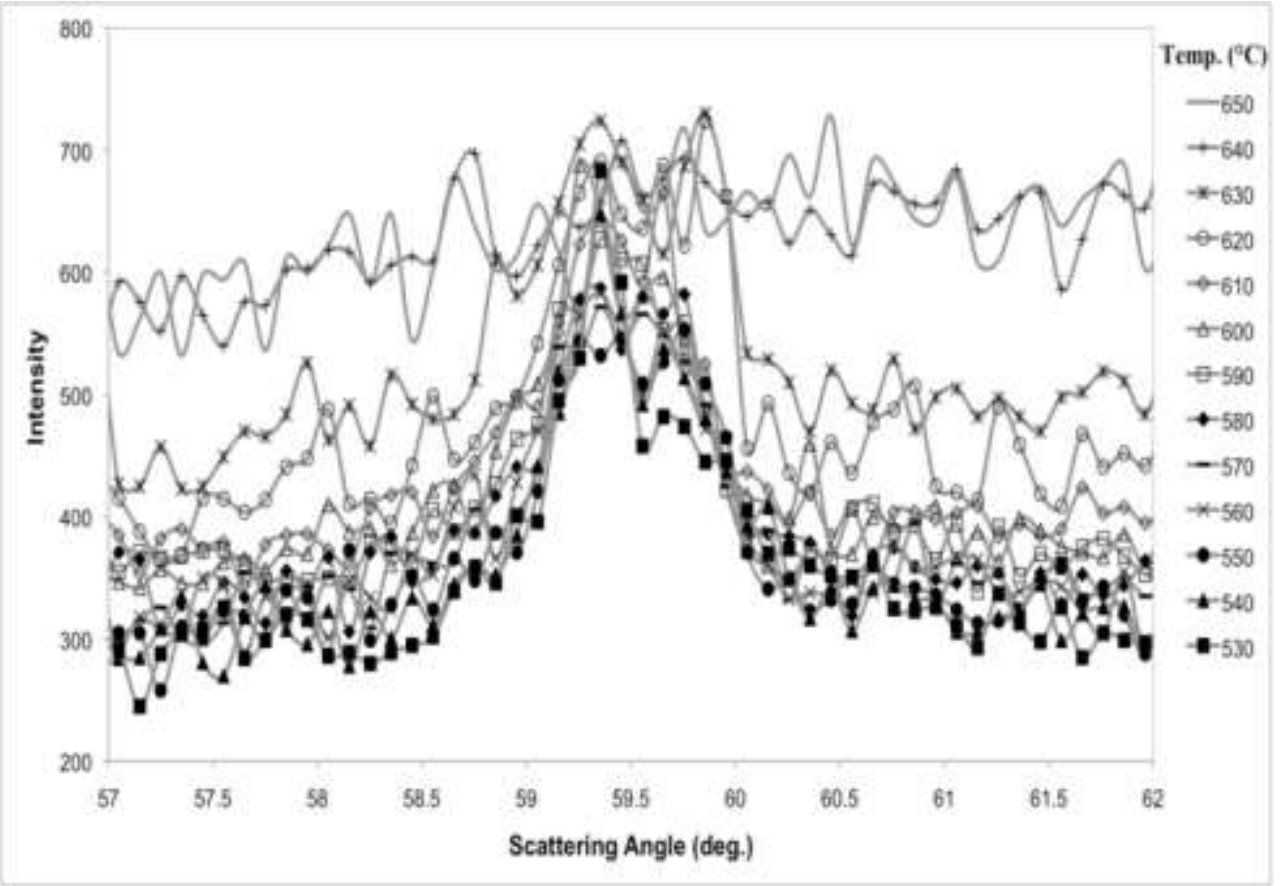

Figure A.6-1. Peak evolution of $\alpha-\mathrm{Al}_{(111)}$ phase in unrefined binary $\mathrm{Al}-5 \mathrm{wt} \% \mathrm{Cu}$ alloy.

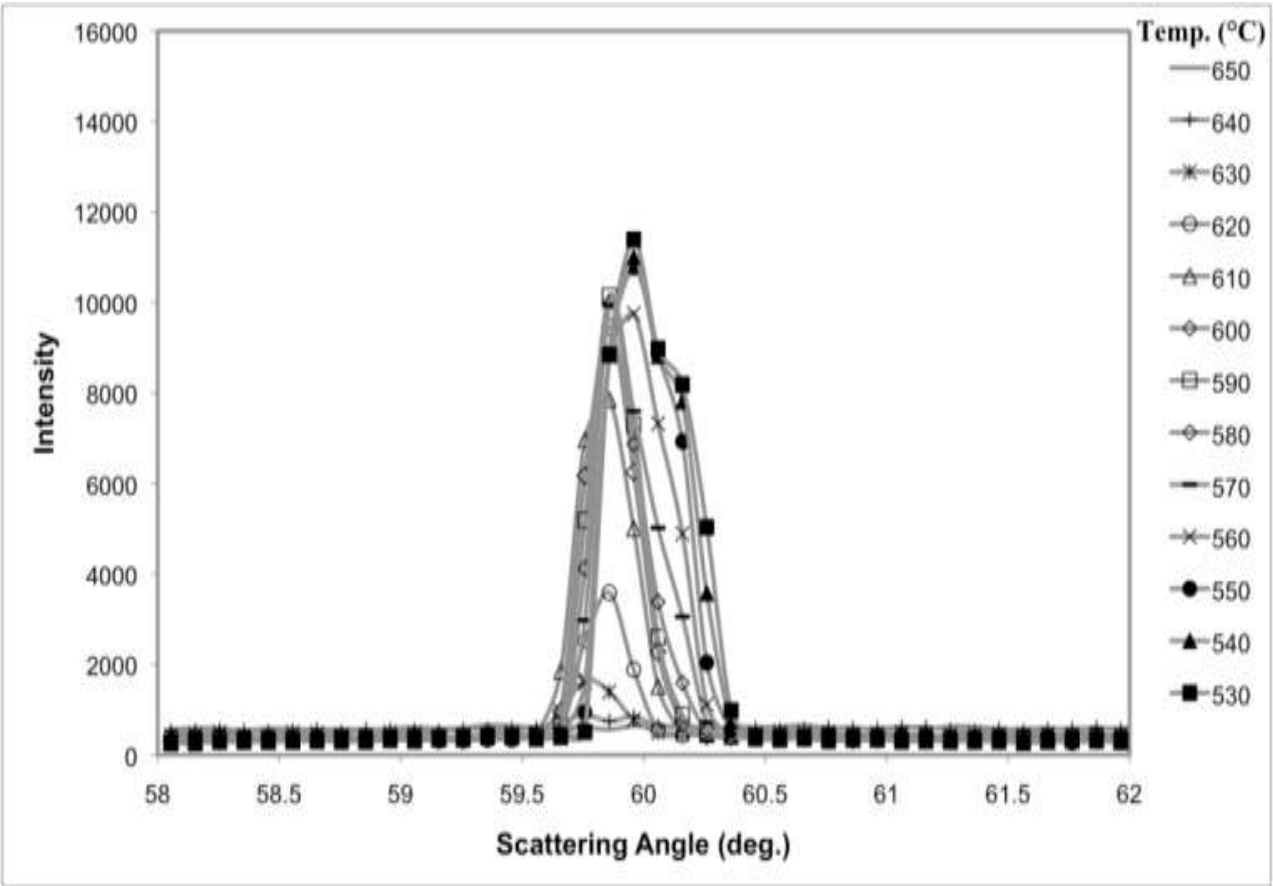

Figure A.6-2. Peak evolution of $\alpha-\mathrm{Al}_{(111)}$ phase in $0.02 \mathrm{wt} \%$ Ti binary Al-5 wt\% $\mathrm{Cu}$ alloy. 


\section{A6.2 Primary $\mathrm{Al}_{(200)}$ Peak Evolution}

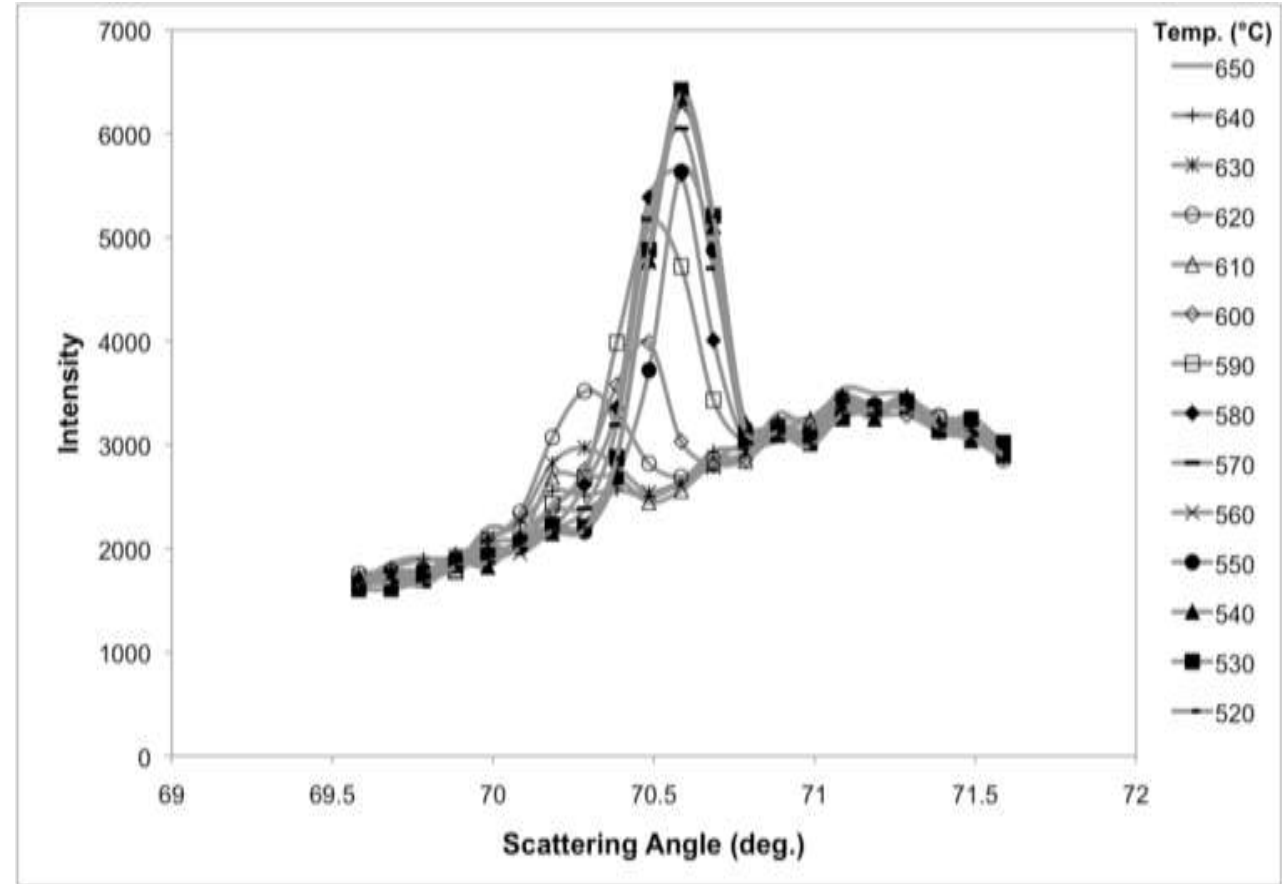

Figure A.6-3. Peak evolution of $\alpha-\mathrm{Al}_{(200)}$ in $0.02 \mathrm{wt} \%$ Ti binary $\mathrm{Al}-5 \mathrm{wt} \% \mathrm{Cu}$ alloy.

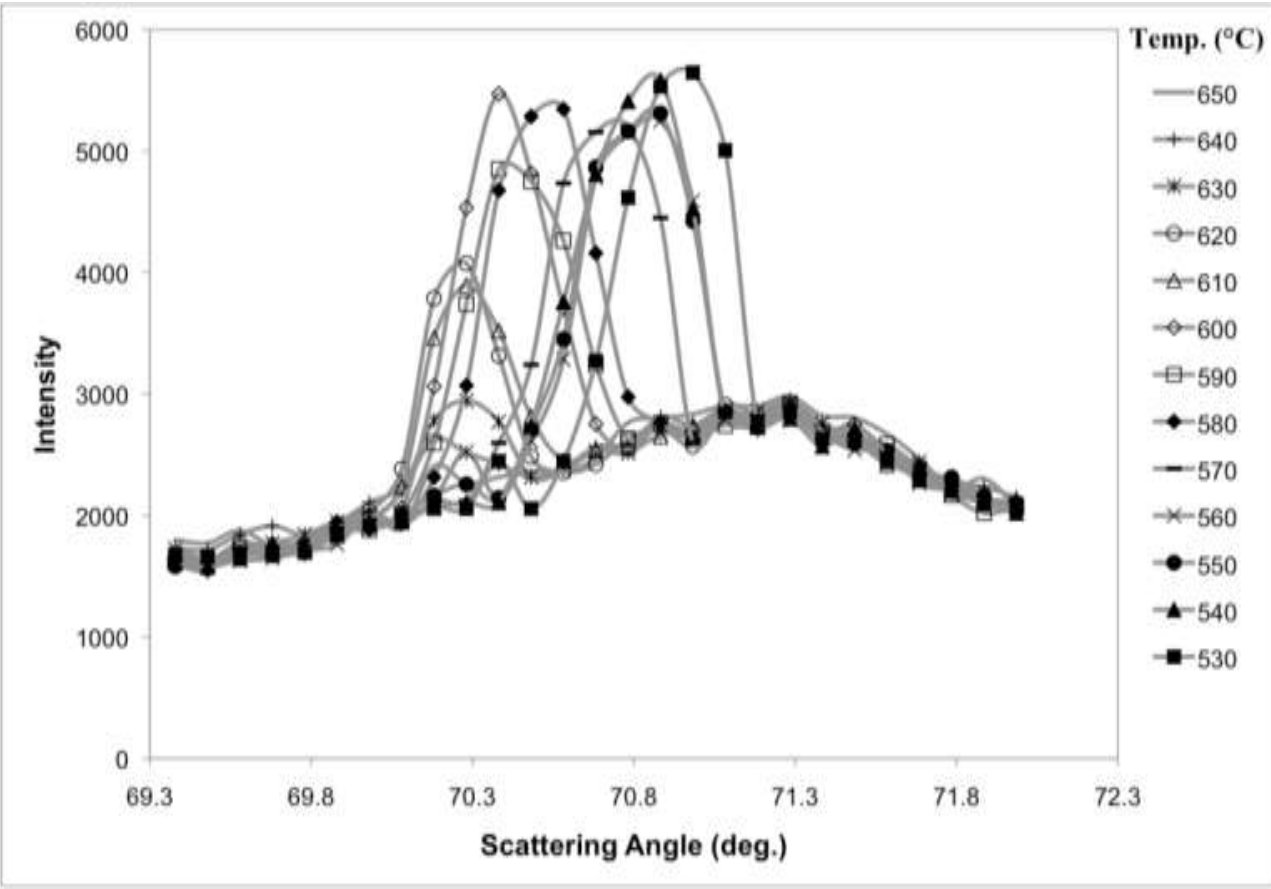

Figure A.6-4. Peak evolution of $\alpha-\mathrm{Al}_{(200)}$ in $0.05 \mathrm{wt} \%$ Ti binary $\mathrm{Al}-5 \mathrm{wt} \% \mathrm{Cu}$ alloy. 


\section{A6.3 Phase Evolution of Primary $\mathrm{Al}_{(200)}$}

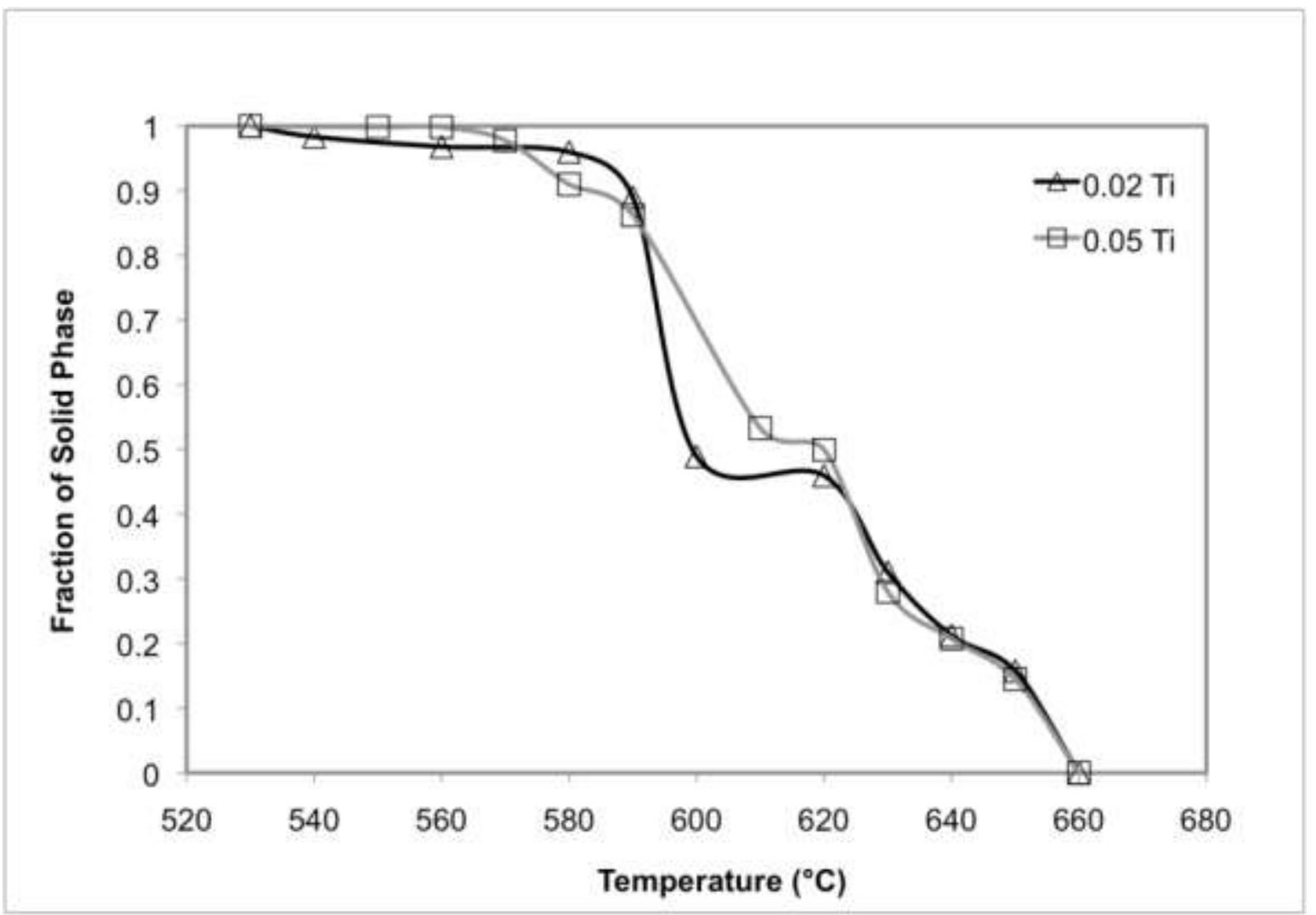

Figure A.6-5. Phase evolution of $\alpha-\mathrm{Al}_{(200)}$ solid solution in the refined binary $\mathrm{Al}-5 \mathrm{wt} \% \mathrm{Cu}$ alloys. 


\section{A6.4 Intermetallic $\mathrm{Al}_{2} \mathrm{Cu}_{(310)}$ Peak Evolution}

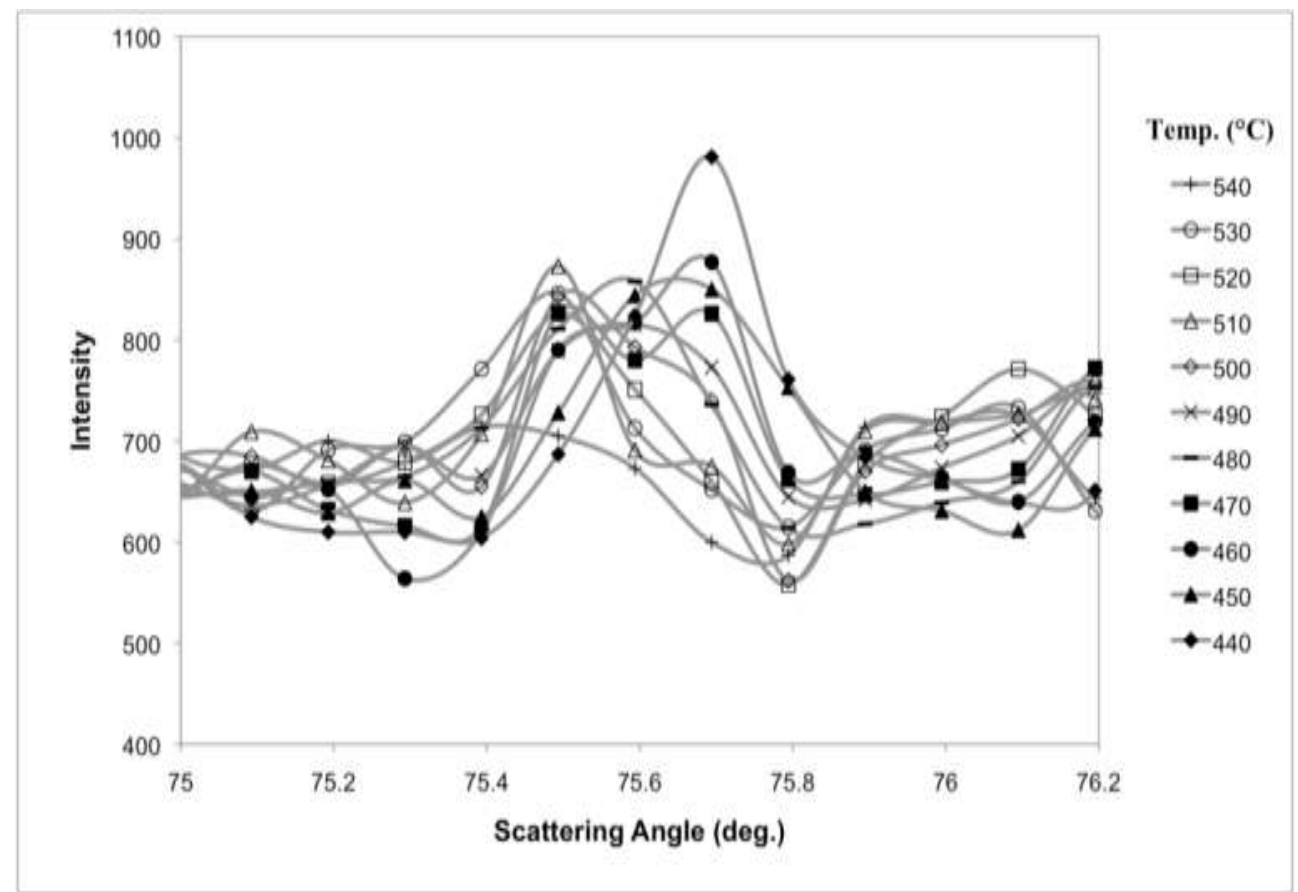

Figure A.6-6. Peak evolution of $\mathrm{Al}_{2} \mathrm{Cu}_{(310)}$ in unrefined binary $\mathrm{Al}-5$ wt $\% \mathrm{Cu}$ alloy.

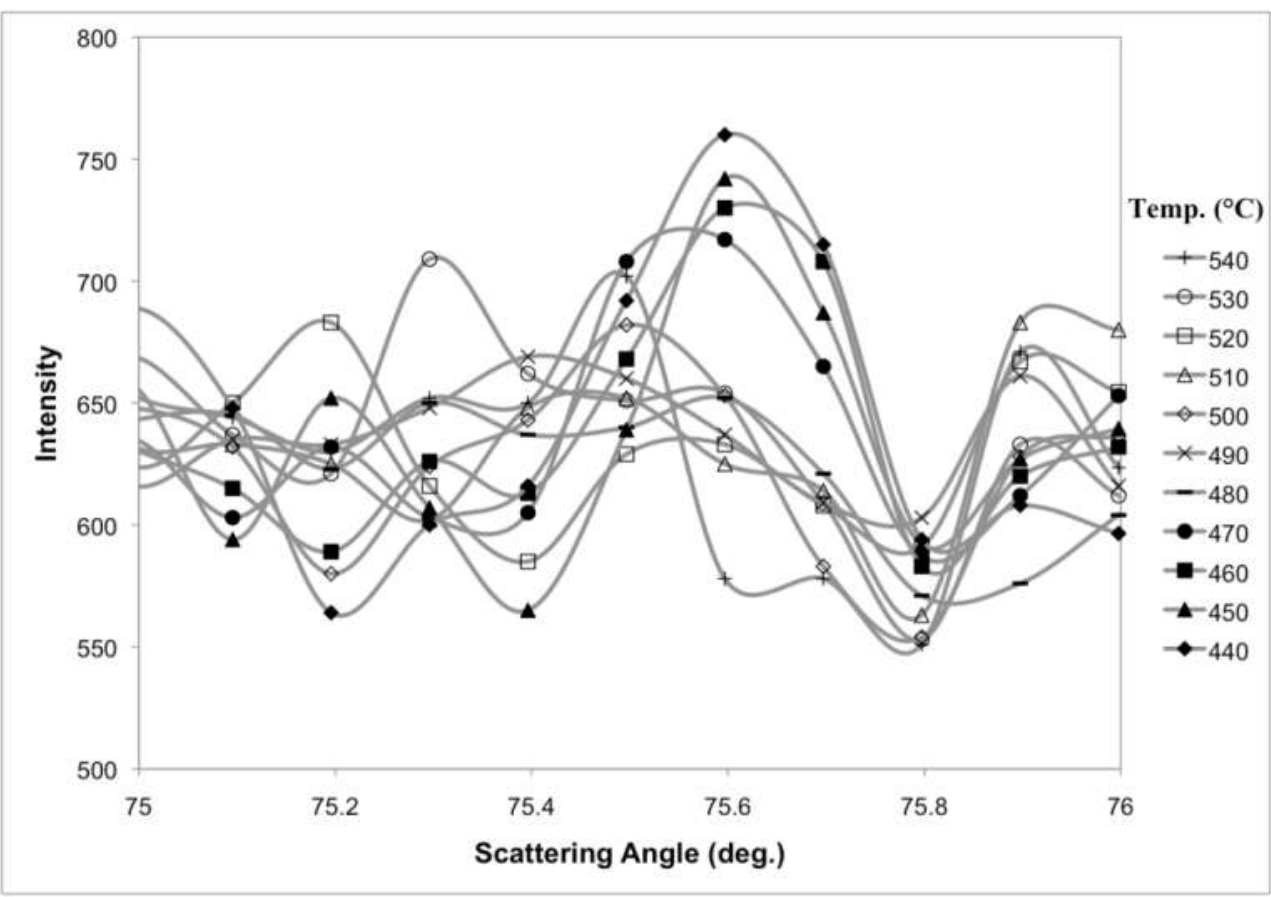

Figure A.6-7. Peak evolution of $\mathrm{Al}_{2} \mathrm{Cu}_{(310)}$ in $0.02 \mathrm{wt} \%$ Ti binary $\mathrm{Al}-5 \mathrm{wt} \% \mathrm{Cu}$ alloy. 
Appendix 7 - List of Academic Achievements 


\section{A7.1 Peer-Reviewed Journal Paper Publications}

1. D'Elia, F., Ravidran, C. and Sediako, D., "Interplay Among Solidification, Microstructure, Residual Strain and Hot Tearing in B206 Aluminum Alloy.” Materials Science and Engineering A. (accepted: August 2014).

2. D'Elia, F., Ravindran, C., Sediako, D. and Donaberger., R., "Solidification Analysis of an Al-5 wt\% Cu Alloy Using In Situ Neutron Diffraction.” Canadian Metallurgical Quarterly. (accepted: July 2014).

3. D'Elia, F., Ravindran, C., Sediako, D., Kainer, K.U. and Hort, N., "Hot Tearing Mechanisms of B206 Aluminum-Copper Alloy." Materials and Design. Vol. 64 (2014): 44-55.

4. Lombardi, A., D'Elia, F., Ravindran, C. and MacKay, R., "Replication of Engine Block Cylinder Bridge Microstructure and Mechanical Properties with Lab Scale 319 Al Alloy Billet Castings.” Materials Characterization. Vol. 87 (2014): 125-137.

5. D'Elia, F., Ravindran, C. and Sediako, D., "Quantification of Residual Strain Associated with Reduction of Hot Tears by Grain Refinement in B206 Aluminum Alloy." Canadian Metallurgical Quarterly. Vol. 53, No. 2 (2014): 151-159.

6. Lombardi, A., D’Elia, F., Ravindran, C., Sediako, D., Murty, B.S. and MacKay, R., "Interplay between Residual Stresses, Microstructure, Process Variables and Engine Block Casting Integrity.” Metallurgical and Materials Transactions A. Vol. 43 (2012): 5258-5270.

7. D'Elia, F., Ravindran, C. and Rao, K.P., "Evaluation of Hot Cracking in AZ91E and AE42 Magnesium Alloys.” Canadian Metallurgical Quarterly. Vol. 51 (2012): 346-355.

8. Lombardi, A., Sediako, D., D’Elia, F., Ravindran, C. and MacKay, R., "Neutron Diffraction Study on Residual Stress in Aluminum Engine Blocks Following Machining and Service Testing.” SAE International Journal of Materials and Manufacturing. Vol. 5 (2012): 115-121.

9. D'Elia F., Ravindran C. and Sediako D., "Effect of Grain Refinement on Residual Strain and Hot Tearing in B206 Aluminum Alloy." Advanced Materials Research. Vol. 409 (2012): 35-40.

10. Lombardi, A., D'Elia, F., Ravindran, C. and MacKay, R, "Variation in Microstructure 
and Mechanical Properties Along the Cylinder Bores of a Modified A319 Al Engine Block with Cast-in Iron Liners.” AFS Transactions. Vol. 119 (2011) Paper No. 11-074 (9 pages).

11. Lombardi, A., D’Elia, F., Ravindran, C., Murty, B.S. and MacKay, R., "Analysis of Secondary Phases in the Microstructure of 319 Type Al Alloy Engine Blocks Using Electron Microscopy and Nano Indentation.” Trans. Indian Institute of Metals. Vol. 64 (2011): 7-11.

12. Sediako, D., D’Elia, F., Lombardi, A., Machin, A., Ravindran, C., Hubbard, C., MacKay, R., "Analysis of Residual Stress Profiles in the Cylinder Web Region of an As-Cast V6 Al Engine Block with Cast-In Fe Liners Using Neutron Diffraction.” SAE International Journal of Materials and Manufacturing. Vol. 4 (2011): 138-151.

13. D'Elia, F. and Ravindran, C., "Poisoning in Grain Refinement of A319 Aluminum Alloy and its Effect on Hot Tearing.” AFS Transactions. Vol. 118 (2010) Paper No. 10-065 (8 pages).

14. Bichler, L., D’Elia, F., Ravindran C. and Sediako D., "Reduction of Hot Tears: Alloy and Casting Process Optimization using Neutron Diffraction.” SAE International Journal of Materials and Manufacturing. Vol. 3, No. 1 (2010): 484-492.

15. D'Elia, F. and Ravindran, C., "Influence of Grain Refinement on Hot Tearing in B206 and A319 Aluminum Alloys". Trans. Indian Institute of Metals. Vol. 62, No. 4-5 (2009): 315-319.

16. D'Elia, F. and Ravindran, C., "Effect of Ti-B Grain Refiner on Hot Tearing in Permanent Mold Cast B206 Aluminum Alloy.” AFS Transactions. Vol. 117 (2009): 139-148.

17. Bichler, L., D'Elia, F. and Ravindran, C., "Reduction of Hot-tears in AZ91D Magnesium Alloy: Effect of Alloy Cooling Rate.” AFS Transactions. Vol. 116 (2008): 795-803.

\section{A7.2 Conference Proceedings}

1. D'Elia, F., Lombardi, A., Ravindran, C., Sediako, D. and Rao, K.P. (2014): Assessment of Hot Cracking During TIG Welding of B206 Aluminum Alloy. TMS Annual Meeting \& Exhibition, San Diego, California, February 16-20, 2014, Light Metals, 195-199.

2. D'Elia, F., Ravindran C. and Rao K.P. (2012): Assessment of Hot Cracking 
Susceptibility in AZ91E Using the Varestraint Test. Proceedings of the $9^{\text {th }}$ International Conference on Magnesium Alloys and their Applications, Vancouver, Canada, July 8-12, 2012, 917-922.

3. Sediako, D., Lombardi, A., D’Elia, F., Ravindran, C., Machin, A., Rogge, R.B. and MacKay, R. (2012): Residual Stress Mapping in the Interbore Region of a Heat Treated Aluminum Engine Block. CIM MetSoc. COM 2011, International Symposium of Light Metals, Montreal, October 2-5, 2011, 331-342.

4. Sediako, D., D’Elia, F., Lombardi, A., Ravindran, C., Machin, A., Hubbard, C. and MacKay, R. (2011): Application of Neutron Diffraction in Analysis of Residual Stress Profiles in the Cylinder Web Region of an As-cast V6 Al Engine Block with Cast-in Fe Liners. TMS Annual Meeting \& Exhibition, San Diego, California, February 27 - March 3, 2011, Supplemental Proceedings: Materials Fabrication, Properties, Characterization and Modeling, 299-308. .

5. D'Elia, F. and Ravindran, C. (2010): Investigating the Effect of Grain Refinement on Hot Tearing in B206 and A319 Aluminum Alloys. CIM MetSoc. COM 2010, International Symposium of Light Metals, Vancouver, October 3-6, 2010, 91-102.

\section{A7.3 Fellowships and Awards}

$3 / 2014$

$11 / 2013$

$9 / 2010-8 / 2013$

$1 / 2013-4 / 2013$

$10 / 2012$

2/2012; 2/2010

$5 / 2011$

$2 / 2011$
Alexander von Humboldt Postdoctoral Fellowship (Tenable at HelmholtzZentrum Geesthacht in Germany from 1/2015 - 12/ 2016)

2013 Materials Science and Technology (MS\&T) Conference - Best

Student Presentation Award (Applied Neutron Scattering in Engineering and Materials Science Research symposium)

Natural Sciences and Engineering Research Council of Canada (NSERC)

Canada Graduate Scholarship - Doctoral

NSERC Michael Smith Foreign Study Supplement

MetSoc Doctoral Student Scholarship

Foundry Education Foundation (FEF) - George Barker Scholarship

AUTO21 HQP Poster Competition - Fifth Place in Canada

Inducted into Alpha Sigma Mu Honour Society 
11/2010 - 1/2011 International Conference and Research Support Fund

12/2010 International Symposium for Research Scholars (ISRS) 2010 - Best

Poster Award

9/2008 - 8/2010 Ontario Graduate Scholarship

$6 / 2010$

AUTO21 HQP Poster Competition - Best in Theme - Fourth Place in

Canada

$2 / 2010$

Non-Ferrous Foundry Society Scholarship

$1 / 2010$

American Foundry Society (AFS) Outstanding Organization Award

(Centre for Near-net-shape Processing of Materials)

$3 / 2009$

American Society for Materials (ASM) Poster Night - Best Poster Award

9/2007 - 8/2008 Ryerson University Graduate Scholarship

5/2007 - 8/2007 NSERC Undergraduate Student Research Award (USRA)

$2 / 2007$

AFS - Ontario Scholarship

$2 / 2007$

FEF Scholarship 


\section{References}

1. Campbell, J. Castings. Oxford: Butterworth-Heinemann, 1991.

2. Gourlay C.M and Dahle, A.K., "The Five Feeding Mechanisms", Shape Casting: The John Campbell Symposium by TMS. (2005): 93-102.

3. Fuoco, R., Correa, E.R. and Andrade Bastos, M., "Effects of Grain Refinement on Feeding Mechanisms in A356 Aluminum Alloy.” AFS Transactions. Vol. 106 (1998): 401-409.

4. Gruzleski, J.E. Microstructure Development During Metalcasting. Des Plaines, Illinois: American Foundrymen's Society, Inc., 2000.

5. Arnberg, L., Backerud, L. and Chai, G. Solidification Characteristics of Aluminum Alloys Volume 3: Dendrite Coherency. Des Plaines, Illinois: American Foundrymen's Society, Inc., 1996.

6. Eskin, D.G., Suyitno and Katgerman, L., "Mechanical Properties in the Semi-solid State and Hot Tearing of Aluminum Alloys." Progress in Materials Science. Vol. 49 (2004): 629-711.

7. Sigworth, G.K., "Hot Tearing of Metals." AFS Transactions. Vol. 104 (1996): 10531062.

8. Li, S. and Apelian, D. "Hot Tearing of Aluminum Alloys: A Critical Literature Review." International Journal of Metalcasting. Vol. 5 (2011): 23-40.

9. Verö, J., “The Hot-Shortness of Aluminum Alloys.” The Metals Industry. Vol. 48 (1936): $431-442$.

10. Singer, A.R.E. and Cottrell, A., "Properties of the Aluminum-Silicon Alloys at Temperatures in the Region of Solidus." Journal of the Institute of Metals. Vol. 73 (1946): 33-54.

11. Pumphrey, W.I. and Jennings, P.H., "A Consideration of the Nature of Brittleness at Temperatures Above the Solidus in Castings and Welds in Aluminum Alloys." Journal of the Institute of Metals. Vol. 75 (1948): 235-256.

12. Bishop, H.F., Ackerlind, C.G. and Pellini, W.S., "Metallurgy and Mechanics of Hot Tearing." AFS Transactions. Vol. 60 (1952): 818-833.

13. Pellini, W.S., "Strain Theory of Hot Tearing.” Foundry. Vol. 80 (1952): 125-199. 
14. Bishop, H.F., Ackerlind, C.G. and Pellini, W.S., "Investigation of Metallurgical and Mechanical Effects in the Development of Hot Tearing." AFS Transactions. Vol. 65 (1957): 247-258.

15. Saveiko, V.N., "Theory of Hot Tearing.” Russian Castings Production. Vol. 11 (1961): 453-456.

16. Pumphrey, W.I. and Lyons, J.V., "Cracking During the Casting and Welding of the More Common Binary Aluminum Alloys.” Journal of the Institute of Metals. Vol. 118 (1948): 439-455.

17. Prokhorov, N.N., "Resistance to Hot Tearing of Cast Metals During Solidification." Russian Castings Production. Vol. 2 (1962): 172-175.

18. Metz, S.A. and Flemings, M.C., "A Fundamental Study on Hot Tearing." $\underline{\text { AFS }}$ Transactions. Vol. 78 (1970): 453-460.

19. Rosenberg, R.A., Flemings, M.C. and Taylor, H.F., "Nonferrous Binary Alloys Hot Tearing.” AFS Transactions. Vol. 69 (1960): 518-528.

20. Niyama, E., "Some Considerations on Internal Cracks in Continuously Cast Steel." Proceedings Japan-US Joint Seminar on Solidification of Metals and Alloys. (1977): 271282.

21. Clyne, T.W. and Davies, G.J., "The Influence of Composition on Solidification Cracking Susceptibility in Binary Alloy Systems.” British Foundryman. Vol. 74 (1981): 65-73.

22. Davies, V. de L., "The Influence of Grain Size on Hot Tearing." British Foundryman. (1970): 93-101.

23. Clyne, T.W. and Davies, G.J., "A Quantitative Solidification Test for Casting and an Evaluation of Cracking in Aluminum-Magnesium Alloys.” British Foundryman. Vol. 68 (1975): 238-244.

24. Spittle, J.A. and Cushway, A.A., "Influences of Superheat and Grain Structure on HotTearing Susceptibilities of Al-Cu Alloy Castings.” Metals Technology. Vol. 10 (1983): 613.

25. Oya, S., Honma, U., Fujii, T. and Othaki, M., "Evaluation of Hot Tearing in Binary Al$\mathrm{Cu}$ and Al-Si Alloy Castings.” Aluminum. Vol. 60 (1984): 777-779.

26. Eskin, D.G. and Katgerman, L., "Effect of Structure on Hot Tearing Properties of Aluminum Alloys.” Materials Science Forum. Vol. 561-565 (2007): 995-998. 
27. Chamberlain, B. and Watanabe, S., "A Natural Aging Aluminum Alloy, Designed for Permanent Mold Use." AFS Transactions. Vol. 85 (1977): 133-142.

28. Sigworth, G.K., Rios, O., Howell, J. and Kaufman, M., "Development Program on Natural Aging Alloys.” AFS Transactions. Vol. 112 (2004): 387-408.

29. Pekguleryuz, M.O. and Vermette, P., "A Study on Hot-Tear Resistance of Magnesium Die Casting Alloys." AFS Transactions. Vol. 114 (2006): 729-736.

30. Srinivasan, A., Wang, Z., Huang, Y., Beckmann, F., Kainer, K.U. and Hort, N., "Hot Tearing Susceptibility of Magnesium-Gadolinium Binary Alloys.” Transactions of the Indian Institute of Metals. (2012) DOI 10.1007/s12666-012-0210-1.

31. Srinivasan, A., Wang, Z., Huang, Y., Beckmann, F., Kainer, K.U. and Hort, N., "Hot Tearing Characteristics of Binary Mg-Gd Alloy Castings." Metallurgical and Materials Transactions A. Vol. 44 (2013): 2285-2298.

32. Wang, Z., Huang, Y., Srinivasan, A., Liu, Z., Beckmann, F., Kainer, K.U. and Hort, N., "Hot Tearing Susceptibility of Binary Mg-Y Alloy Castings." Materials and Design. Vol. 47 (2013): 90-100.

33. Braccini, M., Suéry, M. and Stucky, M., "Influence of Grain Refinement on HotCracking in Aluminum-Copper Alloys." Fonderie Fondeur d'aujourd'hui. Vol. 208 (2001): 12-23.

34. Warrington, D. and McCartney, D.G., "Development of a New Hot-cracking Test for Aluminum Alloys." Cast Metals. Vol. 2 (3) (1989): 134-143.

35. Warrington, D. and McCartney, D.G., "Hot-cracking in Aluminum Alloys 7050 and 7010 - A Comparative Study.” Cast Metals. Vol. 3 (4) (1991): 202-208.

36. Easton, M., Grandfield, J., StJohn, D. and Rinderer, B., "The Effect of Grain Refinement and Cooling Rate on the Hot Tearing of Wrought Aluminum Alloys." Materials Science Forum. Vol. 30 (2006): 1675-1680.

37. Viano, D., StJohn, D., Grandfield, J. and Caceres, C., "Hot Tearing in Aluminum-Copper Alloys.” Light Metals 2005. (2005): 1069-1073.

38. Li, S., Sadayappan, K. and Apelian, D., "Role of Grain Refinement in the Hot Tearing of Cast Al-Cu Alloy." Metallurgical and Materials Transactions B. Vol. 44 (2013): 614-623. 
39. Eskin, D.G., Suyitno, Mooney, J.F. and Katgerman, L., "Contraction of Aluminum Alloys During and After Solidification.” Metallurgical and Materials Transactions A. Vol. 35 (2004): 1325-1335.

40. Eskin, D.G. and Katgerman, L., "Thermal Contraction During Solidification of Aluminum Alloys." Materials Science Forum. Vol. 519-521 (2006): 1681-1686.

41. Kubota, M. and Kitaoka, S., "Solidification Behavior and Hot Tearing Tendency of Aluminum Casting Alloys.” AFS Transactions. Vol. 81 (1973): 424-427.

42. Arnberg, L., Chai, G. and Backerud, L., "Determination of Dendrite Coherency in Solidifying Melts by Rheological Measurements." Materials Science and Engineering A. Vol. 173 (1993): 101-103.

43. Chai, G., "Dendrite Coherency During Equiaxed Solidification in Aluminum Alloys." Chemical Communications. No. 1. Stockholm University (1994).

44. Lin, S., Aliravci, C. and Pekguleryuz, M.O., "Hot-Tear Susceptibility of Aluminum Wrought Alloys and the Effect of Grain Refining." Metallurgical and Materials Transactions A. Vol. 38 (2007): 1056-1068.

45. Bichler, L., Elsayed, A., Lee, K. and Ravindran, C., "Influence of Mold and Pouring Temperatures on Hot Tearing Susceptibility of AZ91D Magnesium Alloy.” International Journal of Metalcasting. Vol. 2, No. 1 (2008): 43-54.

46. Zhen, Z.S., Hort, N., Huang, Y.D., Utke, O., Petri, N. and Kainer, K.U., "Hot Tearing Behaviour of Binary Mg-1Al Alloy Using a Contraction Force Measuring Method." International Journal of Cast Metals Research. Vol. 22, No. 1-4 (2009): 331-334.

47. Sadayappan, K., Sahoo, M., and Weiss, D., "Evaluation of the Hot Tear Susceptibility of Selected Magnesium Casting Alloys in Permanent Molds.” AFS Transactions. Vol. 115 (2007): 761-766.

48. Novikov, I.I. and Grushko, O.E., "Hot Cracking Susceptibility of Al-Cu-Li and Al-CuLi-Mn alloys." Materials Science and Technology. Vol. 11 (1995): 926-932.

49. Instone, S., StJohn, D. and Grandfield, J., "New Apparatus for Characterizing Tensile Strength Development and Hot Cracking in the Mushy Zone." International Journal of Cast Metals Research. Vol. 12, No. 6 (2000): 441-456. 
50. Davidson, C., Viano, D., Lu, L. and StJohn, D., “Observation of Crack Initiation During Hot Tearing." International Journal of Cast Metals Research. Vol. 19, No. 1 (2006): 5965.

51. Cao, G. and Kou, S., "Hot Tearing of Ternary Mg-Al-Ca Alloy Castings." Metallurgical and Materials Transactions A. Vol. 37 (2006): 3647-3663.

52. Cao, G. and Kou, S., "Real-Time Monitoring of Hot Tearing in AZ91E Magnesium Casting.” AFS Transactions. Vol. 115 (2007): Paper No. 07-034.

53. Cao, G., Haygood, I. and Kou, S., "Onset of Hot Tearing in Ternary Mg-Al-Sr Alloy Castings.” Metallurgical and Materials Transactions A. Vol. 41 (2010): 2010-2139.

54. Li, S., Apelian, D. and Sadayappan, K., "Quantitative Investigation of Hot Tearing of Al$\mathrm{Cu}$ Alloy (206) Cast in a Constrained Bar Permanent Mold.” Materials Science Forum. Vol. 618-619 (2009): 57-62.

55. Vogel, S.C. and Carpenter, J.S., "Brief Introduction to Neutron Scattering and Global Neutron User Facilities.” Journal of Metals. Vol. 64, No.1 (2012): 104-111.

56. Squires, G.L. Introduction to the Theory of Thermal Neutron Scattering. Mineola, New York: Dover Publications Inc. (1978): 2-10.

57. Bichler, L., "Phenomenological Studies of Hot Tearing During Solidification of Magnesium Alloys.” PhD thesis, Ryerson University (2009): 1-367.

58. Withers, P.J. and Bhadeshia, H.K.D.H., "Residual Stress Part 1 - Measurement Techniques.” Materials Science and Technology. Vol. 17 (2001): 355-365.

59. Withers, P.J. and Bhadeshia, H.K.D.H., "Residual Stress Part 2 - Nature and Origins." Materials Science and Technology. Vol. 17 (2001): 366-374.

60. Withers, P.J., "Residual Stress and its Role in Failure." Reports on Progress in Physics. Vol. 70 (2007): 2211-2264.

61. Bichler, L., Ravindran, C. and Sediako, D., "Onset of Hot Tearing in AE42 Magnesium Alloy." Canadian Metallurgical Quarterly. Vol. 48, No. 1 (2009): 81-90.

62. Bichler, L., Ravindran, C. and Sediako, D., "Ex Situ Measurement of Strain Associated with Hot Tearing in AZ91D and AE42 Magnesium Alloys Using Neutron Diffraction.” Canadian Journal of Physics. Vol. 88 (2010): 715-721.

63. ISO/TS 21432: Non-destructive Testing-Standard Test Method for Determining Residual Stresses by Neutron Diffraction. 2005-07-15. 
64. D’Elia, F., “A Study on Grain Refinement and Hot Tearing in Permanent Mold Cast Aluminum Alloys." MASc thesis, Ryerson University (2009): 1-96.

65. D'Elia, F. and Ravindran, C., "Effect of Ti-B Grain Refiner on Hot Tearing in Permanent Mold Cast B206 Aluminum Alloy.” AFS Transactions. Vol. 117 (2009): 139-148.

66. Sigworth, G.K., "Fundamentals of Solidification in Aluminum Castings." International Journal of Metalcasting. Vol. 8, No. 1 (2014): 7-20.

67. Callister, Jr., W.D. Materials Science and Engineering: An Introduction. New York, New York: John Wiley \& Sons Inc., 2007.

68. Kamguo Kamga, H., Larouche, D., Bournane, M. and Rahem, A., "Hot Tearing of Aluminum-Copper B206 Alloys with Iron and Silicon Additions." Materials Science and Engineering A. Vol. 527 (2010): 7413-7423.

69. Jones, G.P. and Pearson, J., "Factors Affecting the Grain Refinement of Aluminum Using Titanium and Boron Additives.” Metallurgical Transactions B. Vol. 7 (1976): 223-234.

70. Sigworth, G.K., "The Grain Refining of Aluminum and Phase Relationships in the Al-Ti-B System.” Metallurgical Transactions A. Vol. 15 (1984): 277-282.

71. Guzowski, M.M., Sigworth, G.K and Sentner, D.A., "The Role of Boron in the Grain Refinement of Aluminum with Titanium." Metallurgical Transactions A. Vol. 18 (1987): 603-619.

72. McCartney, D.G., "Grain Refining of Aluminum and its Alloys Using Inoculants." International Materials Reviews. Vol. 34, No. 5 (1989): 247-260.

73. Mohanty, P.S. and Gruzleski, J.E., "Mechanism of Grain Refinement in Aluminum." Acta Metallurgica Materialia. Vol. 34, No. 5 (1995): 2001-2012.

74. Sigworth, G.K., "Communication on Mechanism of Grain Refinement in Aluminum." Scripta Materialia. Vol. 34, No. 6 (1996): 919-922.

75. Murty, B.S., Kori, S.A. and Chakraborty, M., "Grain Refinement of Aluminum and its Alloys by Heterogeneous Nucleation and Alloying." International Materials Reviews. Vol. 47, No.1 (2002): 3-29.

76. Shabestari, S.G. and Ghodrat, S., "Assessment of Modification and Formation of Intermetallic Compounds in Aluminum Alloy Using Thermal Analysis." Materials Science and Engineering A. Vol. 467 (2007): 150-158. 
77. Bäckerud, L., Chai, G. and Tamminen, J. Solidification Characteristics of Aluminum Alloys, Volume 2: Foundry Alloys. Stockholm, Sweden: AFS/SKANALUMINUM, 1990.

78. Bichler, L., Ravindran, C. and Sediako, D., "Neutron Diffraction Measurement of Strain Required for the Onset of Hot Tearing in AZ91D Magnesium Alloy.” Transactions of the Indian Institute of Metals. Vol. 61 (2008): 293-300.

79. Davis, J.R. Aluminum and Aluminum Alloys. Materials Park, Ohio: ASM International, 1993.

80. Clausen, B., Lorentzen, T. and Leffers, T., "Self-Consistent Modelling of the Plastic Deformation of F.C.C. Polycrystals and its Implications for Diffraction Measurements of Internal Stresses.” Acta Materialia. Vol. 46, No. 9 (1998): 3087-3098.

81. Kasprzak, W., Sediako, D., Walker, M., Sahoo, M. and Swainson, I., "Solidification Analysis of an Al-19 Pct Si Alloy Using In Situ Neutron Diffraction." Metallurgical and Materials Transactions A. Vol. 42 (2011): 1854-1862.

82. Hunsicker, H.Y., "Dimensional Changes in Heat Treating Aluminum Alloys." Metallurgical Transactions A. Vol. 11 (1980): 759-773.

83. Lombardi, A., Sediako, D., Ravindran, C. and MacKay, R., "In-Situ Neutron Diffraction Analysis of the Stress-Free d-Spacing during Solution Heat Treatment of Modified 319 Al Alloy Engine Blocks.” Canadian Metallurgical Quarterly. (accepted: July 2014)

84. Fornaro, O. and Palacio, H.A., "Study of Dilute Al-Cu Solidification by Cooling Curve Analysis." Journal of Materials Science. Vol. 44 (2009): 4342-4347.

85. Drezet, J.M., Mireux, B., Szaraz, Z. and Pirling, T., "In Situ Neutron Diffraction During Casting: Determination of Rigidity Point in Grain-Refined Al-Cu Alloys." Materials. Vol. 7 (2014): 1165-1172.

86. Colley, L.J., Wells, M.A., MacKay, R. and Kasprzak, W., "Dissolution of Second Phase Particles in 319-Type Aluminum Alloy." Proceedings of the $26^{\text {th }}$ ASM Heat Treating Society Conference. (2011): 189-198. 